University of Warwick institutional repository: http://go.warwick.ac.uk/wrap

A Thesis Submitted for the Degree of PhD at the University of Warwick

http://go.warwick.ac.uk/wrap/3212

This thesis is made available online and is protected by original copyright.

Please scroll down to view the document itself.

Please refer to the repository record for this item for information to help you to cite it. Our policy information is available from the repository home page. 


\title{
Nucleosome positioning in Arabidopsis
}

\author{
Sarah Louise Usher
}

Thesis submitted in fulfilment of the requirements

for the degree of Doctor of Philosophy

The University of Warwick,

Warwick HRI/Rothamsted Research

September 2009

Dr Graham King (Rothamsted Research)

Dr Smita Kurup (Rothamsted Research)

Dr Guy Barker (Warwick HRI)

This project was carried out under a BBSRC funded strategic studentship. 


\section{Contents}

$\begin{array}{ll}\text { List of Tables } & 7\end{array}$

List of Figures $\quad 9$

$\begin{array}{ll}\text { Acknowledgments } & 13\end{array}$

$\begin{array}{ll}\text { Declaration } & 14\end{array}$

$\begin{array}{ll}\text { Summary } & 15\end{array}$

$\begin{array}{ll}\text { Abbreviations } & 16\end{array}$

$\begin{array}{lr}\text { Preface } & 19\end{array}$

Chapter 1 General Introduction $\quad 20$

1.1 Epigenetics $\quad 21$

1.2 Epigenetic marks $\quad 21$

1.2.1 DNA methylation $\quad 21$

1.2.2 Histone modification $\quad 23$

1.2.3 Chromatin structure at the Nucleolar organiser regions $\quad 24$

1.3 The structure of the nucleosome 25

$\begin{array}{ll}\text { 1.4 Nucleosome Positioning } & 27\end{array}$

1.4.1 Influence of local DNA structure on nucleosome position $\quad 27$

1.4.2 Nucleosome positioning sequence preferences $\quad \mathbf{2 8}$

1.4.3 Nucleosome positioning and gene expression 30

1.5 Chromatin remodelling 32

1.5.1 Translational and rotational nucleosome positioning 32

1.5.2 ATP dependant remodelling complexes 33

1.5.3 Mode of action, twist defect and loop recapture $\quad 34$

1.6 Variation on linker length and higher-order chromatin structure $\quad 36$

1.7 Recent advances and tools for chromatin studies 39

1.8 Nucleosome positioning databases $\quad 42$

1.9 Project aims

1.9.1 Scientific Hypotheses to be tested 43

1.9.2 Experimental approaches $\quad 44$

Chapter 2 General materials and methods 45

2.1 General laboratory protocols $\quad 46$

2.1.i Plant material 44

2.1.ii Formaldehyde cross-linking of protein and DNA 46 
2.1.iii Nuclei isolation $\quad 47$

2.1.iv Preparation of nucleosomal DNA 48

2.1.v Preparation of the enzyme 48

2.1.vi Quantitative Micrococcal Nuclease digests 48

2.1.vii Preparative Micrococcal nuclease digests 49

2.1.viii Cloning $\quad 49$

2.1.ix Determination of clone library quality $\quad 51$

2.1.x Sequencing of clone library $\quad 51$

2.1.xi Large scale sequencing of Arabidopsis nucleosome fragments $\quad \mathbf{5 1}$

2.1.xii Hybridisation to the Affymetrix GeneChip ${ }^{\circledR}$ Arabidopsis 51 Tiling 1.0R Array

2.2 General data analysis protocols $\quad 53$

2.2.i Sequence BLAST alignment $\quad 53$

2.2.ii Paired-end matching $\quad 53$

2.2.iii Extraction of nucleosome sequences $\quad 53$

2.2.iv Detection of periodicity $\quad 54$

2.2.v Kernel Density Estimation $\quad 55$

2.2.vi Construction of nucleosome occupancy files $\mathbf{5 5}$

2.2.vii Description of external datasets $\quad \mathbf{5 8}$

2.2.viii Analysis of tiling array $\quad \mathbf{5 8}$

Chapter 3 The characteristics of Arabidopsis nucleosome sequences 59

3.1 Introduction $\quad 60$

3.1.1 Introduction to nucleosome positioning signals $\quad \mathbf{6 0}$

3.1.2 Aims of study in this chapter $\quad 63$

3.2 Methods $\quad 64$

3.2.1 Determination of end-dinucleotides at the site of MNase activity 64

3.2.2 Calculation of observed/expected ratios for dinucleotides at sites of MNase activity

3.2.3 Calculation of GC content

3.2.4 Determination of dinucleotide frequencies $\quad 65$

3.2.5 Distribution of dinucleotides along the nucleosome DNA 66 fragment 
3.2.6 Periodicity of dinucleotide distribution

3.2.7 Construction of a nucleosome sequence database

3.3 Results

68

3.3.1.i Generation of Arabidopsis nucleosome DNA fragments 68

3.3.1.ii Dinucleosome DNA fragments from a clone library 68

3.3.1.iii Dinucleosome DNA fragments sequenced by 454 FLX technology

3.3.1.iv Nucleosome DNA fragments sequenced using Illumina (Solexa) technology

3.3.2.i Specificity of micrococcal nuclease 74

3.3.2.ii The GC content of nucleosomal DNA fragments 78

3.3.2.iii Dinucleotide occurrence in nucleosome DNA fragments 81

3.3.2.iv Dinucleotide distribution within Arabidopsis nucleosome DNA fragments

3.3.2.v Periodicity of dinucleotides in Arabidopsis nucleosome DNA fragments

3.3.3.i Nucleosome Sequence database 94

3.4 Discussion 96

3.4.1 Micrococcal nuclease sequence preferences 96

3.4.2 Nucleosome DNA fragment GC content 97

3.4.3 Nucleosome DNA fragment dinucleotide occurrence 98

3.4.4 Distributions of dinucleotides within nucleosome DNA 99

3.4.5 Summary 100

Chapter 4 Nucleosome spacing and linker length variation 102

4.1 Introduction 103

4.1. Introduction to linker DNA and linker length variation $\mathbf{1 0 3}$

$\begin{array}{ll}\text { 4.2 Methods } & 108\end{array}$

4.2.1 Calculation of linker length $\quad 108$

$\begin{array}{ll}\text { 4.2.2 Calculation of length periodicities } & 108\end{array}$

4.2.3 Determination of Arabidopsis exon and intron length and $\quad 109$ periodicity

4.2.4.i Determination of linker length within an Arabidopsis nucleolar 109 organiser region 
4.2.4.iii Kolmogorov-Smirnov test $\quad \mathbf{1 1 0}$

4.3 Results $\quad 112$

4.3.1 Linker length distribution $\quad 112$

4.3.1.i Calculation of linker length from dinucleosome DNA 112 fragments

4.3.1.ii Derived linker lengths from mononucleosome positions $\quad \mathbf{1 1 4}$

$\begin{array}{ll}\text { 4.3.2 Periodicity in linker length distribution } & 117\end{array}$

4.3.3 Relationship between linker length and intron length 119

4.3.4 Linker length variation in an Arabidopsis nucleolar organiser $\quad \mathbf{1 2 3}$ region

4.4 Discussion

127

4.4.1 Arabidopsis linker length distribution $\quad \mathbf{1 2 7}$

4.4.2 Periodicity with linker length distribution in Arabidopsis $\quad 129$

4.4.3 Relationship between linker length and intron length 130

4.4.4 Effect of linker length on higher-order chromatin structure $\quad 131$

4.4.5 Nucleosome positioning in the nucleolar organiser region in $\mathbf{1 3 3}$ Arabidopsis

4.4.6 Structure of the rRNA regions on Chromosomes 2 and $3 \quad 134$

4.4.7 Summary 136

Chapter 5 Nucleosome distribution and patterns of occupancy 137

$\begin{array}{ll}5.1 \text { Introduction } & 138\end{array}$

5.1.2 Aims of study in this chapter $\quad 139$

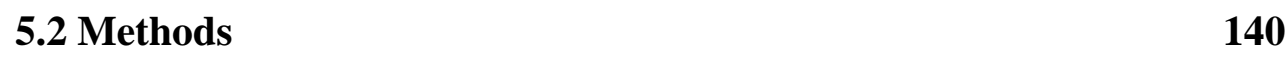

5.2.1 Genomic representation in datasets and distribution of fragment $\mathbf{1 4 0}$

5.2.2 Genomic patterns of nucleosome occupancy $\quad \mathbf{1 4 0}$

5.2.3 Distribution of occupancy with exons and introns 141

5.2.4 Nucleosome occupancy around transcriptional start sites 142

5.2.5 Kernel Density Estimation 143

5.3 Results $\quad 144$

5.3.1 Genomic distribution and coverage of nucleosome sequences $\quad \mathbf{1 4 4}$

$\begin{array}{ll}\text { 5.3.2 Trends of nucleosome occupancy } & 148\end{array}$

$\begin{array}{ll}\text { 5.3.2.i Genomic trends of nucleosome occupancy } & 148\end{array}$ 
5.3.2.ii Nucleosome occupancy in specific chromosomal regions

5.3.2.iii Nucleosome occupancy within coding vs. non-coding regions 153

5.3.3 Nucleosome positioning around transcriptional start sites $\mathbf{1 5 5}$

5.3.3.i Nucleosome presence at the transcriptional start site (TSS) 155

5.3.3.ii Nucleosome occupancy around transcriptional start sites $\quad \mathbf{1 5 6}$

5.3.4 Nucleosome occupancy around the Arabidopsis nucleolar $\mathbf{1 6 0}$ organiser region

5.4 Discussion $\quad 165$

5.4.1 Genomic trends of nucleosome distribution $\quad 165$

5.4.2 Nucleosome occupancy in 5' region of PHERES1 and $\mathbf{1 6 6}$ PHERES2

5.4.3 Nucleosome occupancy within exons and introns $\quad 167$

5.4.4 Nucleosome occupancy at the transcriptional start site $\quad \mathbf{1 6 8}$

5.4.5 Nucleosome occupancy with the Arabidopsis nucleolar 169 organiser regions

5.4.6. Summary 170

Chapter 6 Effect of DNA methylation on nucleosome position 172

$\begin{array}{ll}\text { 6.1 Introduction } & 173\end{array}$

$\begin{array}{ll}\text { 6.2 Methods } & 175\end{array}$

$\begin{array}{ll}6.3 \text { Results } & 175\end{array}$

6.3.1 Nucleosome occupancy and DNA methylation within the $\quad \mathbf{1 7 5}$

Arabidopsis nucleolar organiser region (NOR)

6.4 Discussion $\quad 179$

$\begin{array}{ll}\text { 6.4.1 Summary } & 180\end{array}$

$\begin{array}{ll}\text { Chapter } 7 \text { General discussion } & 181\end{array}$

7.1 Nucleosome positioning in Arabidopsis 182

7.2 Differences in nucleosome position resulting from loss of CG $\mathbf{1 8 5}$ methylation

$\begin{array}{ll}\text { 7.3 Higher-order chromatin structure } & 188\end{array}$

$\begin{array}{ll}7.4 \text { Technological advances } & 189\end{array}$

$\begin{array}{ll}7.5 \text { Conclusions and future work } & 190\end{array}$

$\begin{array}{lr}\text { Chapter } 8 \text { References } & 192\end{array}$ 


\section{List of Tables}

Table 2.1 A list of PERL scripts generated and used in this study.

Table 2.2 Annotation codes assigned to each bp position in Arabidopsis in construction of nucleosome occupancy files.

Table 2.3 A description with references of the publically available datasets used in this thesis.

Table 3.1 Sources of publicly-available nucleosome sequence data collected and used to populate a nucleosome sequence database.

Table 3.2 Arabidopsis datasets generated in this study.

Table 3.3 A summary of datasets derived from Ath_03_wt and Ath_04_MET1.

Table 3.4 Frequency of [W], [WG] and [WC] at the site of MNase activity for each nucleosome dataset.

Table 3.5 Observed/expected ratios of dinucleotide occurrence at the site of MNase activity for nucleosome sequences of nucleosome sequences.

Table 3.6 Mean, standard deviation and median of the distribution of GC content (\%) of wt_left and MET1_mono nucleosome sequences.

Table 3.7 Statistically significant periodicities calculated by Fourier analysis for each complementary pair of dinucleotides for the nucleosome datasets wt_left, MET1_mono,Ath_02_wt and Ath_rand.

Table 3.8 The periodicities of the linker datasets: wt_left_linker and MET1_left_linker.

Table 3.9 A list of the species represented in the Nucleosome Sequence database, collected from a variety of sources, and the number of entries for each species.

Table 3.10 A summary of some of the large nucleosome position datasets generated by high-throughput, next generation technologies, which are available, at the time of writing, for data comparisons and analysis.

Table 4.1 The two subsets extracted from the Ath_03_wt dataset which represent the annotated rRNA regions on Chromosomes 2 and 3

Table 4.2 Linker lengths calculated from Arabidopsis dinucleosome datasets. 
Table 4.3 Mean, median and standard deviation of linker lengths derived from mononucleosome positions using the datasets Ath_O2_wt and Ath_04_MET1.

Table 4.4 Statistically significant periodicities calculated by Fourier analyses and PACF for linker length distributions derived from Arabidopsis datasets.

Table 4.5 The mean, standard deviation and median lengths for Arabidopsis exons, introns, intron start to exon end and from intron start to the next intron end.

Table 4.6 Mean, median, standard deviation and periodicity (Fourier analysis) of datasets Ath_03_rna2 and Ath_03_rna3.

Table 4.7 Kurtosis and skewness values for the smoothed distributions of linker lengths from the Ath_03_rna2 and Ath_03_rna3 datasets.

Table 4.8 A list of linker lengths for different species collected from literature.

Table 5.1 Percent of sequences from datasets Ath_03_wt, Ath_04_MET1 and Ath_05_wt which align to each chromosome.

Table 5.2 Percent of occupied bases for each chromosome for the datasets Ath_03_wt,Ath_04_MET1 and Ath_05_wt.

Table 5.3 Gene models present in a selected region (1) on chromosome 1

Table 5.4 Gene models present in a selected region (2) on chromosome 1

Table 5.5 Exon/intron ratios of nucleosome occupancy and average occupancy/base within different annotation classes on chromosome 1, for datasets the Ath_03_wt, Ath_04_MET1 and Ath_05_wt.

Table 5.6 The presence of nucleosomes at, and upstream of TSSs within chromosome 1 for the datasets: Ath_03_wt and Ath_04_MET1. 


\section{List of Figures}

Figure 1.1 Diagram showing the sites of post-translational modification of the histone tails of the 4 core histones.

Figure 1.2 The nucleosome core particle, linker DNA and linker-associated histone.

Figure 1.3 Representation of the organisation of periodic DNA sequence patterns around the histone octomer.

Figure 1.4 Schematic diagram to show the possible remodelling mechanisms.

Figure 1.5 Schematic representations of the different models of chromatin fibre folding.

Figure 1.6 A Chart showing the relative numbers of organisms represented in the NRPD.

Figure 2.1 Schematic showing the order of assignment of gene annotation to each Arabidopsis nucleotide. The annotation is re-written in successive rounds until all annotations are added.

Figure 3.1 Entity-relationship diagram showing tables and fields of the relational nucleosome sequence database.

Figure 3.2 Schematic showing the processing of Arabidopsis dinucleosome fragments from dataset Ath_02_wt.

Figure 3.3 Distributions of dinucleosome DNA fragment lengths.

Figure 3.4 Schematic showing the process of paired-end Illumina sequencing of Arabidopsis nucleosome DNA fragments.

Figure 3.5 Length distributions of mono and di nucleosome sequences of datasets.

Figure 3.6 Schematic showing the division of dinucleosome sequences from datasets Ath_03_wt and Ath_04_MET1 into nucleosome and linker, and the generation of derived linker datasets.

Figure 3.7 Observed/expected ratios for the occurrence of each dinucleotide at the site of MNase activity for Arabidopsis datasets.

Figure 3.8 Distribution of GC content (\%) for each sequence in the Arabidopsis datasets.

Figure 3.9 Mean observed/expected ratios of the occurrence of each dinucleotide for each DNA sequence in the nucleosome Arabidopsis datasets. 
Figure 3.10 Mean observed/expected ratios of the occurrence of each dinucleotide for each DNA sequence in the linker Arabidopsis datasets.

Figure 3.11 Distribution of $[\mathrm{AA} / \mathrm{TT}]$ dinucleotides within nucleosome DNA fragments.

Figure 3.12 Distribution of $[\mathrm{CC} / \mathrm{GG}]$ dinucleotides along the nucleosome DNA fragments.

Figure 3.13 Distribution of $[\mathrm{AA} / \mathrm{TT}]$ dinucleotides and $[\mathrm{CC} / \mathrm{GG}]$ dinucleotides within the Ath_rand dataset.

Figure 3.14 Distribution of dinucleotides along the linker DNA fragments of wt_left_linker and MET1_left_linker.

Figure 3.15 The twice-difference distribution of the [AA/TT] dinucleotide frequencies along nucleosome DNA fragments for the dataset $w t_{-} l e f t$.

Figure 3.16 Periodogram constructed by Fourier analysis of the [AA/TT] dinucleotide distribution for the $w t \_l e f t$ dataset.

Figure 4.1 Organisation of the $18 \mathrm{~S}, 5.8 \mathrm{~S}$ and $25 \mathrm{~S}$ rRNA genes, the internal transcribed spacers and intergenic spacer.

Figure 4.2 Schematic showing the method for estimation of linker length from mononucleosome DNA datasets.

Figure 4.3 Schematic showing the method for calculating exon and intron lengths from intron position data and for calculating intron/exon combination datasets.

Figure 4.4 Schematic showing the calculation of linker length by subtracting $2 \mathrm{x}$ $147 \mathrm{bp}$ (two nucleosomes) from the length of a dinucleosome DNA fragment.

Figure 4.5 Distributions of calculated linker lengths from dinucleosome-length sequences from the datasets Ath_01_wt, Ath_03_wt and Ath_04_MET1.

Figure 4.6 Schematic showing the method for inferring linker lengths from a set of mononucleosome DNA fragments.

Figure 4.7 Distribution of the inferred linker lengths from datasets Ath_O2_wt and Ath_04_MET1.

Figure 4.8 Schematic showing how lengths of exons, introns, 5'intron-exon3' and 5'intron-exon-intron3' were determined. 
Figure 4.9 Distributions of the length of Arabidopsis genomic components, exons, introns, the distances from intron start to exon end and the distance from intron start to the next intron end

Figure 4.10 Distribution of Arabidopsis first intron lengths.

Figure 4.11 Distribution of Linker lengths for datasets Ath_03_rna2 and Ath_03_rna3.

Figure 4.12 Probability density plots for datasets Ath_03_rna2 and Ath_03_rna3.

Figure 4.13 Cumulative distribution function plot for datasets Ath_03_rna2 and Ath_03_rna3.

Figure 4.14 Demonstration of the possible relationship between a model of the structure of the $30 \mathrm{~nm}$ fibres at increasing linker lengths and Arabidopsis linker length calculated from the dinucleosome sequences of the Ath_04_MET1 dataset.

Figure 4.15 Structure of the annotated rRNA regions on Chromosomes 2 and 3.

Figure 5.1 Schematic showing the method for calculating the distance between the positions of nucleosome fragments aligned to the Arabidopsis reference gnome.

Figure 5.2 Schematic showing the assignment of nucleosome occupancy scores to each position within the Arabidopsis chromosome.

Figure 5.3 Schematic showing the method for determination of the distance between the TSS and the nearest upstream nucleosome boundary.

Figure 5.4 Schematic showing the method for determination of the distance between the TSS and every nucleosome midpoint within $1000 \mathrm{bp}$.

Figure 5.5 The density of nucleosome sequence fragment start co-ordinates within chromosome 1 for datasets Ath_02_wt, Ath_03_wt and Ath_04_MET1, plotted with a 10,000 bp bin size.

Figure 5.6 The density of nucleosome sequence fragment start co-ordinates within a close-up of a $2 \mathrm{Mbp}$ region (boxed in Figure 5.5), within chromosome 1 for datasets Ath_O2_wt, Ath_03_wt and Ath_04_MET1, plotted with a $1,000 \mathrm{bp}$ bin size.

Figure 5.7 Nucleosome occupancy of datasets Ath_04_MET1, Ath_03_wt, and Ath_05_wt, over a 14,500 bp region (region 1) within chromosome 1.

Figure 5.8 Nucleosome occupancy of datasets Ath_04_MET1, Ath_03_wt, and Ath_05_wt, over a 28,000 bp region (region 2) within chromosome 1 . 
Figure 5.9 Schematic showing the method for determination of the distance between the TSS and the nearest upstream nucleosome boundary.

Figure 5.10 Schematic showing the method for calculating the frequency of nucleosome occupancy around the TSSs.

Figure 5.11 Nucleosome occupancy around the TSS of the first $6 \mathrm{Mbp}$ of the forward strand of chromosome 1.

Figure 5.12 Kernal Density Estimation of nucleosome occupancy around the TSSs within the first $6 \mathrm{~Kb}$ of chromosome 1 (forward strand).

Figure 5.13 Alignment of rRNA genes on chromosomes 2 and 3 and nucleosome occupancy of datasets Ath_04_MET1, Ath_05_wt, and Ath_03_wt.

Figure 5.14 Nucleosome occupancy of the rRNA regions on chromosome 2 for datasets Ath_04_MET1,Ath_05_wt and Ath_03_wt.

Figure 5.15 Nucleosome occupancy of the rRNA regions on chromosome 3 for datasets Ath_04_MET1,Ath_05_wt and Ath_03_wt.

Figure 5.16 The nucleosome occupancy around the TSSs of Arabidopsis, yeast and Drosophila.

Figure 6.1 Position of 5me-C relative to nucleosome positions over the rRNA region on chromosome 2.

Figure 6.2 Position of 5me-C relative to nucleosome positions over the rRNA region on chromosome 3.

Figure 6.1 Position of $5 \mathrm{me}-\mathrm{C}$ relative to nucleosome positions over the rRNA region on chromosome 2 .

Figure 6.2 Position of $5 \mathrm{me}-\mathrm{C}$ relative to nucleosome positions over the rRNA region on chromosome 3 . 


\section{Acknowledgements}

I would like to thank the following people, firstly my academic supervisors, Dr. Graham King, Dr. Smita Kurup and Dr. Guy Barker, for all of their support, help and encouragement throughout the $\mathrm{PhD}$. I would also like to thank Paul Verrier and Stephen Powers for help with bioinformatics and statistics. Thanks to all who have helped in my journey who have not been specifically mentioned. Also, thanks to my fellow $\mathrm{PhD}$ students and members of the research group at Rothamsted who provided a strong sense of community, and much useful advice and motivation. Thanks to the BBSRC for funding this research. 


\section{Declaration}

This thesis is the sole work of the author, and no part has previously been published or presented for another degree.

The DNA sequence datasets used throughout this thesis were processed by Graham King as described within Chapter 2.

Warwick HRI's departmental guidelines were used in the preparation of this thesis. 


\section{Summary}

The aim of this project was to test hypotheses relating to nucleosome positioning in Arabidopsis to provide a basis for better understanding of epigenetic transcriptional regulation in plants. Prior to this study, virtually no information existed regarding nucleosome positioning in plants. Eukaryote chromosomes consist of chromatin, composed of nucleosomes separated by linker DNA of variable lengths. Nucleosomes consist of $147 \mathrm{bp}$ of DNA wrapped 1.7 times around a histone octamer. Whilst no consensus nucleosome positioning DNA sequence exists, sequence preferences influence positioning, and contribute to the complex epigenetic processes which act to control transcriptional activity. These details of the underlying mechanisms are known to differ between the plant and animal kingdoms.

High-throughput sequencing technologies were utilised to generate large datasets of mono- and di-nucleosome sequences from wild-type Arabidopsis. These enabled genome-wide analysis and inference of plant-specific patterns of nucleosome positioning and sequence properties. Further data were generated from a methyltransferase antisense (MET1) which is depleted in methylated CG epigenetic marks.

The internal distributions of dinucleotides within Arabidopsis nucleosomes were similar to those observed in non-plant eukaryotes. A unique periodicity in the distribution of linker lengths was detected in Arabidopsis wild type chromatin. In contrast, the METl antisense line displayed the expected periodicity, indicating systematic differences in chromatin organisation. There was a significant increase in nucleosome occupancy within exons compared with introns. However, this difference was less marked in the METI antisense. Specific patterns of nucleosome phasing were observed around transcription start sites. Linker lengths within rRNA gene clusters associated with nucleolar organiser regions (NORs) differed depending on chromosome of origin, suggesting differences in higher order chromatin structure between the NORs. Comparison of the nucleosome position and DNA methylation within the rRNA gene cluster revealed interesting differences between the two regions, which may reflect interactions affecting chromatin structure and transcriptional regulation. 


$\begin{array}{ll}\text { Abbreviations } \\ { }^{\circ} \mathrm{C} & \text { degrees Celsius } \\ { }^{5 \mathrm{~m}} \mathrm{C} & \text { 5-methylcytosine } \\ \mathrm{ACF} & \text { Autocorrelation function } \\ \text { Ath } & \text { Arabidopsis thaliana } \\ \text { BLAST } & \text { Basic Local Alignment Search Tool } \\ \text { bp } & \text { Base pairs } \\ \text { ChiP } & \text { chromatin immunopreciptitation } \\ \text { Chr } & \text { Chromosome } \\ \text { CL } & \text { Confidence limit } \\ \text { Col } & \text { Columbia } \\ \text { CSV } & \text { Comma separated variable } \\ \text { DNA } & \text { Deoxyribonucleic acid } \\ \text { EDTA } & \text { Ethylenediaminetetraacetic acid } \\ \text { F } & \text { Forward } \\ \text { FISH } & \text { Fluorescence in-situ hybridisation } \\ \text { g } & \text { grams } \\ \text { IGR } & \text { Intergenic region } \\ \text { Inr } & \text { Initiator } \\ \text { IPTG } & \text { Isopropyl } \beta \text {-D-1-thiogalactopyranoside } \\ \text { ITS } & \text { Internal transcribed spacer } \\ \text { IUPAC } & \text { The International Union of Pure and Applied Chemistry } \\ \text { KDE } & \text { Kernel Density Estimation } \\ \text { M } & \text { Molar } \\ \text { m } & \text { milli (10-3 }) \\ \text { miRNA } & \text { Micro-RNA } \\ \text { Mbp } & \text { Mega basepairs } \\ \text { MNase } & \text { Micrococcal nuclease } \\ \text { MTE } & \text { motif ten element } \\ n & \text { Number } \\ \text { NASC } & \text { Nottingham A. thaliana Stock Centre } \\ \text { NCBI } & \text { National Centre for Biotechnology Information } \\ \text { NFR } & \text { Nucleosome free region } \\ & \end{array}$




\begin{tabular}{|c|c|}
\hline NOR & Nucleolar organiser region \\
\hline NPRD & Nucleosome Position Region database \\
\hline NRL & Nucleosome repeat length \\
\hline $\mathrm{nt}$ & Nucleotide \\
\hline PACF & Partial autocorrelation function \\
\hline $\mathrm{PcG}$ & Polycomb group \\
\hline Pol I & RNA polymerase I \\
\hline Pol II & RNA polymerase II \\
\hline $\mathrm{R}$ & Reverse \\
\hline RNA & Ribonucleic acid \\
\hline rRNA & Ribosomal ribonucleic acid \\
\hline SNP & Single nucleotide polymorphism \\
\hline snRNA & Small nuclear RNA \\
\hline snoRNA & Small nucleolar RNA \\
\hline tRNA & Transfer RNA \\
\hline TAIR & The Arabidopsis Information Resource \\
\hline TE (buffer) & Tris EDTA buffer \\
\hline Tr.E & Transposable element \\
\hline TSS & Transcriptional start site \\
\hline$\mu$ & micro $\left(10^{-6}\right)$ \\
\hline $\mathrm{U}$ & units \\
\hline url & Uniform resource locator \\
\hline UTR & Untranslated region \\
\hline $\mathrm{V}$ & volts \\
\hline vs. & versus \\
\hline WT & Wild-type \\
\hline $\mathrm{W}$ & Nucleotides \\
\hline X-gal & 5-bromo-4-chloro-3-indolyl-b-D-galactopyranoside \\
\hline $\bar{y}$ & Arithmetic mean \\
\hline
\end{tabular}


IUPAC nucleotide codes were adopted throughout this thesis.

\begin{tabular}{c|c}
\hline IUPAC code & Base abbreviation \\
\hline A & A \\
C & C \\
G & G \\
T & T \\
M & A or C \\
R & A or G \\
W & A or T \\
S & C or G \\
Y & C or T \\
K & G or T \\
V & A, C or G \\
H & A, C or T \\
D & A, G or T \\
B & C, G or T \\
N & A, C, G or T \\
\hline
\end{tabular}




\section{Preface}

The thesis is presented in eight chapters. The background literature and motivation for the project is outlined in Chapter 1. Methods used in more than one results chapter are described in the general materials and methods Chapter 2 Each results chapter follows the convention of presentation as a paper, reviewing relevant literature, describing specific materials and methods, and presenting and discussing findings.

Chapter 3 presents the characteristics of Arabidopsis nucleosome DNA sequences isolated in this project. The aim of this chapter was to determine any sequence preferences in Arabidopsis with respect to nucleosome positioning.

Chapter 4 presents linker length variation in Arabidopsis, with the aim of determining any patterns within the distribution of linker lengths.

Chapter 5 Presents the patterns of nucleosome occupancy within the Arabidopsis genome with respect to regulatory regions. The aim of this chapter is to determine any species-specific nucleosome occupancy.

Chapter 6 presents the position of DNA methylation and nucleosome positioning over the rRNA region, with the aim of determining any relationship between epigenetic marks.

Chapter 7 the results of the thesis are discussed in terms of questions raised, and potential future work.

References are given in Chapter 8. 
Chapter 1

General introduction 


\section{General Introduction}

\subsection{Epigenetics}

Epigenetics can be defined as heritable, reversible modifications to chromatin which affect phenotype and gene expression, but which do not affect the underlying DNA sequence. The term 'epigenetics' (epi meaning over or above), was coined by Conrad Waddington following his work on the development of Drosophila wing morphology and cellular differentiation in development (Waddington, 1953).

Since then, epigenetic phenomena have been widely observed throughout eukaryotes and generally are associated with regulatory mechanisms that affect phenotype, which relate to chromatin structure (Tchurikov, 2005). Chromatin can be thought of as existing in two states: i) heterochromatin which is compact and generally transcriptionally silent and ii) euchromatin which is more relaxed and generally associated with transcriptional activity. Specific epigenetic marks are associated with each class of chromatin (Fransz et al., 2006).

\section{$\underline{1.2 \text { Epigenetic marks }}$}

Chromatin structure is influenced through DNA methylation of cytosine residues, and modification of histone protein N-terminal tails. Such modifications are stable through mitotic and/or meiotic cell divisions giving rise to a heritable activation state (Zilberman and Henikoff, 2005).

\subsubsection{DNA methylation}

DNA methylation is one of the most abundant epigenetic marks, with $5.26 \%$ of all genomic cytosines methylated in Arabidopsis (Lister et al., 2008). DNA methylation occurs at CG, CNG and CNN in plants and mammals (Lister et al., 2009). The pericentromeric regions, repeats and transposable elements have been found to be highly methylated in Arabidopsis. In addition methylation is found in the promoters of developmentally regulated genes and in the gene body of one third of all constitutively expressed genes in Arabidopsis (Cokus et al., 2008; Zhang et al., 2006). DNA methylation is initiated and maintained through DOMAINS 
General introduction

REARRANGED METHYLTRANSFERASE 2 (DRM2), METHYLTRANSFERASE1 (MET1), and CHROMOMETHYLASE 3 (CMT3). MET1 and DRM2 share homology with the mammalian methyltransferases DNA (cytosine-5-)-methyltransferase 1 (DNMT1) and DNA (cytosine-5-)-methyltransferase 3 (DNMT3), respectively while CMT3 is plant-specific. Both MET1 and CMT3 are responsible for maintaining established DNA methylation marks and DRM2 is responsible for establishing new methylation marks (de novo methylation).

The DNA-maintenance methyltransferase MET1 methylates the 5' carbon of cytosine residues in the $\mathrm{CG}$ context, using s-adenosylmethionine as the donor for the methyl group (Cheng, 1995). Mutants in met-1 have drastically reduced levels of CG methylation, and display a variety of developmental abnormalities (Finnegan et al., 1996). While, up regulation of transposable elements was not significant in the met1 or $c m t 3$ single mutants it was significantly higher in the met-1 cmt 3 double mutant, suggesting that the two enzymes may act redundantly to silence transposable elements (Kato et al., 2003b).

The plant-specific CMT3 has been shown to interact, through the chromodomain, with the tails of histone $\mathrm{H} 3$ when it is methylated at both lysines at position 9 and 27 (Lindroth et al., 2004). Therefore, CMT3 may be involved in silencing of homeotic genes such as FLOWERING LOCUS C (FLC) (Bastow et al., 2004).

De novo DNA methylation in Arabidopsis occurs through the DRM1 and 2 DNA methyltransferases. DNA methylation by DRM 1 and 2 is directed by siRNAs. Experiments involved the transformation of Arabidopsis plants with FWA. FWA is silenced in the wild-type, but remains active in the $d r m 1$ and $d r m 2$ mutants and in the siRNA pathway rna-dependant rna polymerase 2, dicer-like3, sde4 and argonaute 4 mutants (Chan et al., 2004). Thus the repression of FWA is through siRNA directed DNA methylation. 


\subsubsection{Histone modification}

Histones are globular proteins with N-terminal tails that are available for modification. Post-translational modifications of histone $\mathrm{N}$-terminal tails within nucleosome have an impact on transcriptional activity and chromatin structure. The pattern of histone modification is referred to as the histone code, since specific epigenetic marks are often associated with specific chromatin structures and transcriptional states (Figure 1.1) (Henikoff et al., 2004). Histones are mostly modified at the N-terminal tails by classes of enzymes such as histone methyltransferases (HMTs) and histone acetyl-transferases (HATs) (Grant, 2001). These enzymes are required both for de novo modification, and maintenance of epigenetic marks. Histone modification appears to be a more complicated mechanism than DNA methylation, the resultant action being dependent on the type and location of modification. Histone modification enzymes contain domains that recognise epigenetic marks; chromodomain containing proteins (such as Heterochromatin Protein 1, HP1) recognise methylation while bromodomain containing proteins (such as DDM1) recognise acetylated histones. These proteins seek out marks and target those areas for further modification resulting in a cascade of modification along a chromosomal region.

In Arabidopsis, flowering is dependent on down regulation of FLC following vernalisation. Levels of FLC remain low during development suggesting an epigenetic mechanism but studies have shown that there is no change in DNA methylation levels before and after vernalisation, there is however a change in the methylation state of histone H3. Before vernalisation, Histone H3 in di-methylated lysine 4, which has been recognised as a transcriptionally active mark. Following vernalisation methylation at this site is reduced in conjunction with di-methylation of Histone $\mathrm{H} 3$ at lysines 9 and 27. Methylation at these sites triggers binding of chromodomain containing HP1 and induce heterochromatin formation and hence silencing. Low levels of FLC are maintained throughout development by expression of VRN1 and 2. VRN1 encodes a DNA binding protein and VRN2 encodes a homologue of the Drosophila SU(Z)12 a member of the Polycomb group Proteins (PcG) family of proteins which have been implicated in maintaining the silence of Drosophila HOX genes through mitotic cell divisions (Bastow et al., 2004). 
General introduction

Di- and tri- methylation of histone $\mathrm{H} 3$ at lysine 9 has been shown to be associated with the promoters of silenced genes in Arabidopsis. The floral repressor FLC is down-regulated following vernalisation by $V R N 1$ and $V R N 2$, allowing floral development. Following cold treatment, VRN2, a homologue of the Drosophila Polycomb-group (PcG) E(Z), is recruited to the 5' region of FLC and causes dimethylation of H3K9 (Bastow et al., 2004).

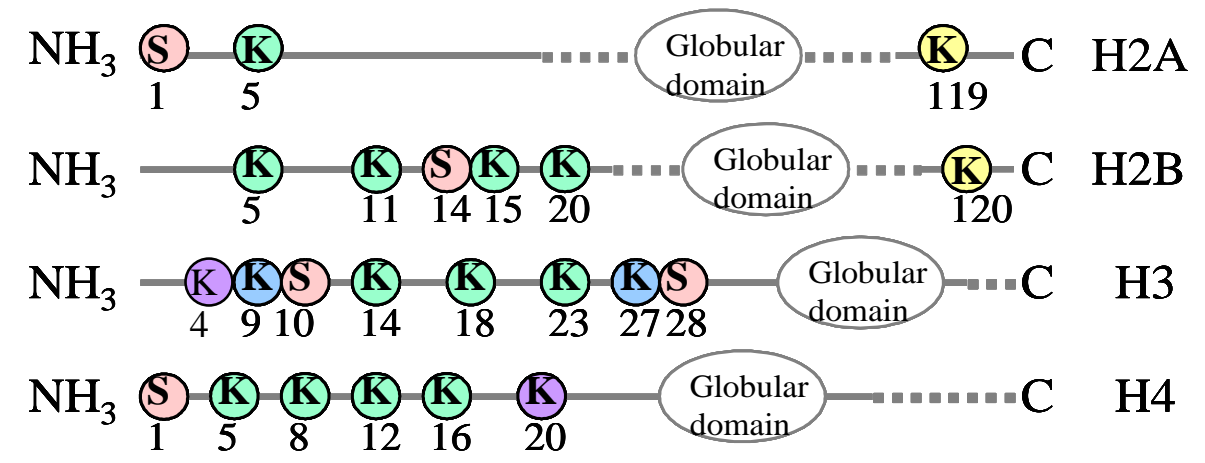

Figure 1.1 Diagram showing the sites of post-translational modification of the histone tails of the 4 core histones. Pink circles represent sites of phosphorylation, green: represents sites of acetylation, yellow: represents sites of ubiquitination, purple: sites of methylation and blue: sites of acetylation and methylation. The type of post-translation modification depends on the transcriptional activity of the DNA within the region of the nucleosome.

\subsubsection{Chromatin structure at the Nucleolar organiser regions}

The nucleolar organiser regions in Arabidopsis, are clustered into regions on chromosomes 2 and 4. They consist of the 18S, 5.8S and 25S rRNA genes in two clusters (nucleolar organiser regions (NORs)) of $700 \pm 130$ tandemly repeated copies on chromosomes 2 and 4, with a single repeat present on chromosome 3 (European Union Chromosome 3 Arabidopsis Genome Sequencing Consortium, 2000). The rRNA genes are separated by two internal transcribed spacer sequences and repeats are separated by an intergenic spacer The coding sequences are transcribed by RNA polymerase I (Pol I) and rRNA transcripts constitute 50-80 \% of transcribed RNA in eukaryotic cells, although transcription of only a subset of repeats is needed to fulfil this requirement (Pruess and Pikaard, 2007). While the histone modification and DNA methylation status are being studied, there is little known about the chromatin structure of these regions. 


\subsection{The structure of the nucleosome}

Nucleosomes are the building blocks of chromatin, and are generally positioned every 157- $240 \mathrm{bp}$ in eukaryote chromatin (Englehardt, 2007). Nucleosomes consist of a core particle, a linker-associated histone H1 (or sometimes H5) and linker DNA (Figure 1.2). The core particle consists of 147bp DNA (Davey and Richmond, 2002) wrapped in a left-hand superhelical turn (of $10.17 \mathrm{bp}$ per turn), 1.67 times around the histone octamer, with the central 129 of $147 \mathrm{bp}$ organised over the histone (Richmond and Davey 2003).

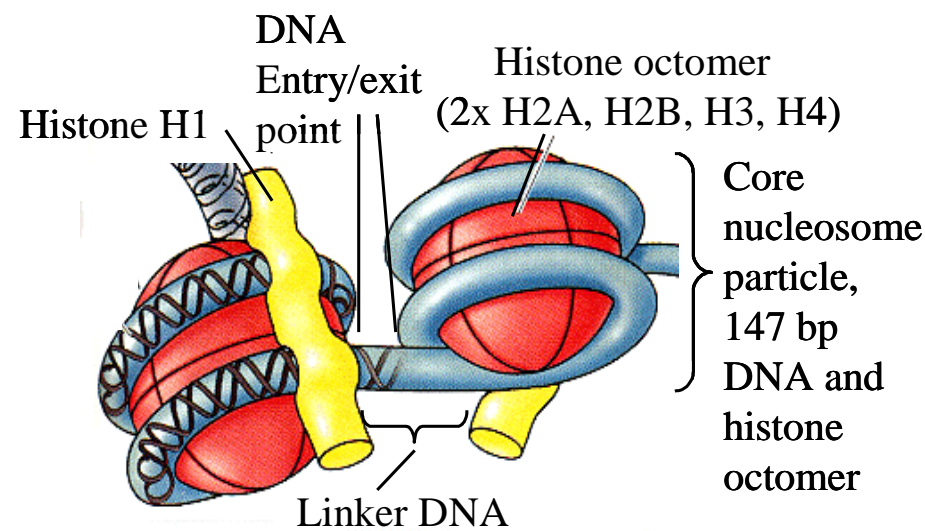

Figure 1.2. The nucleosomal core particle, linker DNA and linker-associated histone. Adapted from Purves et al., (1997)

Evidence from x-ray crystallography shows that the 147 bp nucleosome DNA is not bent uniformly around the histone, but has sharp bends at specific points. Nucleosome DNA is 1.3 times more likely to be bent into the major groove, with the major groove facing in towards the histone octamer. Specific points of interaction exist between the nucleosomal DNA and the histone (Rhodes 1997, Richmond et al., 1984) and the interaction between the histone octamer and the DNA is strongest around the dyad-axis region, with a weaker interaction up to $60 \mathrm{bp}$ from the dyad and a 5 bp periodicity in contact points (Hall et al., 2009).

The histone octamer consists of two molecules of each of histone H2A, H2B, H3 and $\mathrm{H} 4$ proteins, organised into pairs of $\mathrm{H} 3 / \mathrm{H} 4$ and $\mathrm{H} 2 \mathrm{~A} / \mathrm{H} 2 \mathrm{~B}$ dimers. Histone $\mathrm{H} 1$ is associated with linker DNA and protects the DNA at the entry/exit point to the 
General introduction

nucleosome. H1 also aids stability and prevents slippage of the nucleosomal position (Bustin et al., 2005). Linker histone has also been shown to have a greater affinity for methylated DNA and contributes to chromatin compaction (McArthur and Thomas 1996).

Nucleosomes that form at the active centromere contain a centromere-specific variant of histone H3, CENP-A (also called CenH3) (Bernad et al., 2009). Study of Drosophila nucleosomes has revealed that these variants, rather than being targeted towards the centromere, occur at other sites in the chromosome, but are later removed and replaced by canonical histone H3 (Dalal et al., 2007a). The structure of centromeric nucleosomes differs from the majority in that they consist of a histone tetramer rather than an octamer. The nucleosome core consists of one copy of each Histone H2A, H2B, CENP-A and H4 and is more susceptible to enzymatic digestion. Electron microscopy had provided further evidence for small particles, separated by long linkers that resist condensation under physiological conditions (Dalal et al., 2007b). In fact the nucleosome repeat length of the small particles does not seem to differ from that of canonical nucleosomes. 


\subsection{Nucleosome Positioning}

Almost any stretch of DNA has the ability to form nucleosomes. The use of prokaryote DNA in nucleosome reconstitution experiments demonstrates this effect, as prokaryote DNA is not packaged in the same way as eukaryotic DNA in vivo and is not associated with histones (Trifonov, 1984). Reports of preferential positioning, nucleosome-free regions and different nucleosome formation potentials of coding and non-coding DNA suggest that nucleosome formation is affected by specific nucleotide sequences or their associated properties (Vinogradov, 2005; Anselmi et al., 1999). In addition, nucleosomes tend to be positioned on DNA with a higher GC content that the genomic average (Chung and Vingron, 2008).

Although nucleosome positioning is not dependant on specific base recognition by histone proteins, the location of a nucleosome is influenced by a number of factors. These include inherent mechanical properties of the sequence, such as the intrinsic curvature and flexibility, which are determined by base composition of local DNA sequence (Zuccheri et al., 2001). Binding of non-histone proteins, such as those for recombination and transcription, compete with histone proteins for binding sites. Boundary effects and steric hindrance between adjacent nucleosomes are also implicated in positioning as there is a minimum length of linker DNA between nucleosomes that is required to enable formation of higher order structures (Baldi et al., 1996).

Chromatin is a dynamic system where nucleosomes are formed at, and move toward sites of least resistance (low free energy) (Widlund et al., 1999). Nucleosomes tend to be positioned at sites with strong nucleosome positioning signals and require energy (ATP) to be remodelled. These sites in the genome offer enhanced stability and have a higher probability of occupancy by a nucleosome (Fitzgerald and Anderson, 2004).

\subsubsection{Influence of local DNA structure on nucleosome position}

While specific contact points exist between the histone octamer and the nucleosomal DNA, there is no sequence recognition by the histone octamer to serve as a signal for nucleosome positioning. Nucleosome formation is influenced by local DNA structure determined by local DNA sequence, with particular contributions from constituent 
dinucleotides (Fitzgerald and Anderson, 1999). DNA curvature and flexibility are thought to be major determinates of nucleosome positioning (Kiyama and Trifonov, 2002). The curvature and flexibility of a DNA molecule are determined by base sequence (Scipioni et al., 2002; Pina et al., 1990). Intrinsically curved DNA is curved in solution regardless of the presence or absence of protein (Cloutier and Widom, 2004). Flexible DNA, whilst intrinsically straight, is able to deviated from straight and follow different paths.

In order to establish the relationship between DNA structural properties and nucleosome position, DNA sequences have been synthesised and analysed for their ability to form nucleosomes in vitro (Shrader and Crothers, 1989). Sequences containing 5 repeats of a $10 \mathrm{bp}$ sequence [TATAAACGCC] were shown to have the highest affinity for nucleosome formation by competitive reconstitution experiments with bulk DNA and natural nucleosome positioning sequences. However, this DNA sequence was later shown to be unable to position a nucleosome in vivo. This suggests optimal that DNA structure parameters considered in the original experiments are not sufficient alone to position nucleosomes in vivo (Tanaka et al., 1992).

\subsubsection{Nucleosome positioning sequence preferences}

Sequence analysis of nucleosome DNA fragments has identified what appears to be nucleosome positioning sequence preferences. One of the most studied nucleosome positioning patterns is the [AA/TT] dinucleotide motif (Ioshikhes et al., 1996). The [AA] and [TT] dinucleotides tend to be placed counter-phase to each other, (with 5 bp intervals) and were discovered in experimentally mapped nucleosomal sites to be approximately 5 bp apart and occur with a $10.3(+/-0.2)$ bp periodicity, (Figure 1.3). However this counter-phase pattern has since been demonstrated to decay over approximately $50 \mathrm{bp}$, whereas the in-phase [AA/TT] dinucleotide repeating pattern is longer-range, decaying over approximately 200 bp (Cohanim et al., 2006). While the $[\mathrm{AA} / \mathrm{TT}]$ repeating pattern has been well characterised, nucleotide repeats with different base compositions have also been identified. For example, similar dinucleotide patterns consisting of a more general $[\mathrm{RR} / \mathrm{YY}]$ repeating motif have been discovered using sequences of clones from over 1000 human dinucleosome DNA fragments (Kato et al., 2005, Kogan et al., 2006). Further analyses revealed 
General introduction

that the dinucleotide repeat $[\mathrm{CC} / \mathrm{GG}]$ appears to be the dominant nucleosome positioning signal in human chromatin, while the [AA/TT] repeat appears to have little influence (Kogan et al., 2006; Kogan and Trifonov, 2005).

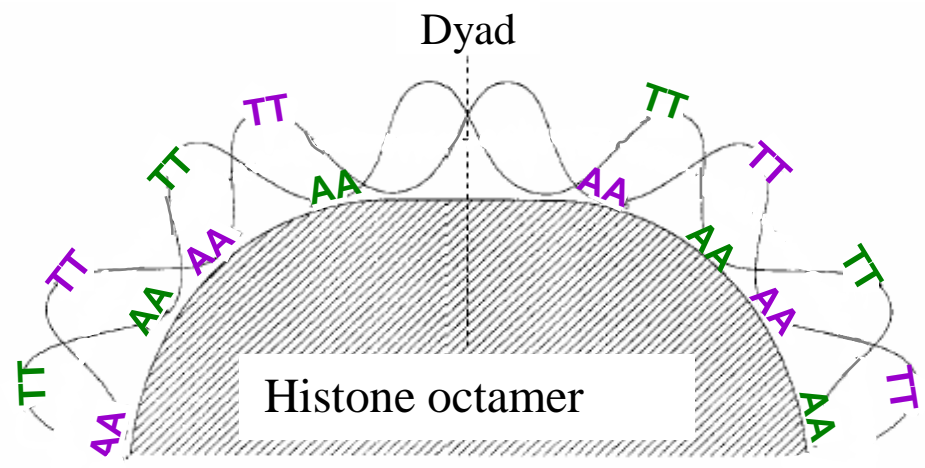

Figure 1.3 Representation of the organisation of periodic DNA sequence patterns around the histone octamer. Positions of dinucleotides on opposite strands are marked in purple and green. Adapted from Ioshikhes et al., (1996)

A trinucleotide motif [VWR] with a 10.5 bp periodicity was identified whilst training a computer program to identify splice site junctions in human DNA (Baldi et al., 1996). This was later confirmed as a nucleosome-positioning signal in yeast (Stein and Bina, 1999). The sequence occurs periodically throughout the human genome. In experiments to identify strong nucleosome positions in mouse chromatin, a $10 \mathrm{bp}$ sequence [TATAA(A/C)CG(T/C)C] was isolated which has the strongest affinity for nucleosome positioning identified to date (Widlund et al., 1997). This sequence was identified following multiple rounds of competitive reconstitution experiments.

A different class of nucleosome positioning motif, not previously described, was discovered in the DNA sequence of the unicellular organism Trichomonas vaginalis. This organism is known to have short 5' and 3' UTRs and a large number of duplicated genes. A 120.9 bp periodicity was discovered, which was more pronounced in gene sequences, and which started between the 5'UTR and the coding sequences with a preference for GC at the third codon, and a weaker preference at the first codon. The repeating 120.9 bp pattern decreased towards the 3 ' ends of the genes, suggesting a preference for nucleosome positioning within genic regions in this organism (Chen et al., 2008). 
General introduction

Sequences containing [TGGA] repeats have been shown to impair nucleosome formation through successive nucleosome reconstitution experiments, where the unreconstituted DNA is selected (Cao et al., 1998). DNA fragments containing [TGGA] repeats (and fragments containing runs of the dinucleotide steps in [TGGA]) are thought to form secondary structures detrimental to nucleosome formation. These motifs occur in tandem repeats throughout eukaryotic genomes and are thought to be important as positioning signals for nucleosomal arrangement. For example, size polymorphisms of tandem [TGGA] repeats at the 5' end of the human myelin basic protein have been associated with subsets of multiple sclerosis cases (Tienari et al., 1998). Other motifs thought to impair nucleosome formation include $[\mathrm{CCG}]$ repeats (ref) and poly $[\mathrm{dA} / \mathrm{dT}]$ tracts (ref).

\subsubsection{Nucleosome positioning and gene expression}

During transcription, 5' ends of transcribed regions must be made available to the transcriptional machinery, whilst downstream sequences may remain unavailable. Nucleosomes positioned at promoter regions influence gene transcription, and relocation of nucleosomes has a dramatic effect on transcription rates (Parthasarthy and Gopinathan, 2006). Nucleosome occupancy is depleted at the promoter regions of many transcriptionally active genes: for example, positioned nucleosomes at promoter regions of polymerase II transcribed genes in Salmonella phage 6 inhibit transcription (Lorch et al., 1987). However, once transcription has been initiated, RNA polymerase is able to 'read-through' nucleosomes positioned in the coding regions. This is thought to be because of the space required at the promoter regions for the transcriptional machinery.

The PHO5 promoter in yeast has been extensively characterised with respect to nucleosome position during transcriptional activation (Boeger et al., 2003, Boeger et al., 2004, Boeger et al., 2005). During transcriptional activation, two of the four nucleosomes positioned at the promoter are lost. Incomplete removal of nucleosomes at the promoter suggests a mechanism of removal and re-formation following the passage of the transcriptional machinery (Boeger et al., 2004). Microarray analysis of yeast chromatin following heat-shock revealed that $65 \%$ of genes had their transcript level altered by a factor of two, and also showed changes in nucleosome 
General introduction

occupancy. Nucleosome occupancy was found to be inversely proportional to the level of transcriptional activity. Furthermore, genes which became active following heat shock showed a loss of nucleosome occupancy at the promoter, while genes which became silenced gained nucleosome occupancy at the promoter (Lee et al., 2004). It was hypothesised that nucleosome depletion at promoters of actively transcribed genes is a characteristic of eukaryotic chromatin structure.

Histone variants H2A.Z have been identified in global studies of yeast chromatin. Nucleosomes containing H2A.Z typically flank a nucleosome-free region, which extends approximately $200 \mathrm{bp}$ upstream of the transcriptional start and includes the promoter region (Raisner and Madhani, 2006). Histone variant H2A.Z is thought to be a mark of euchromatin in yeast as it is not present at promoters of inactive genes. A genome-wide map of H2A.Z-containing nucleosomes revealed the presence of H2A.Z at DNA methylation-depleted regions in Arabidopsis (Zilberman et al., 2008). A peak in H2A.Z occupancy was observed in the position of the first nucleosome after the transcriptional start site in Arabidopsis chromatin, which is consistent with the position of H2A.Z peaks observed in yeast (Mavrich et al., 2008b), Drosophila (Mavrich et al., 2008a) and in transcriptionally active human chromatin (Schones et al, 2008). The genome-wide study in Arabidopsis revealed a lack of H2A.Z-containing nucleosomes from heavily methylated regions such as the pericentromeric regions and transposable elements, suggesting that DNA methylation excludes deposition, or perturbs formation of H2A.Z-containg nucleosomes. Conversely, a mutation in the PIEl domain of the Swrl complex, responsible for deposition of H2A.Z-containing nucleosomes, resulted in genome-wide hypermethylation within gene bodies, suggesting that H2A.Z also protect the DNA from methylation (Zilberman et al., 2008). These results suggest that H2A.Zcontaining nucleosomes are a feature of transcriptional activity at Pol II promoters across kingdoms.

While nucleosome positioning at promoter regions provides a barrier to transcription for some genes, transcription rates of RNA III polymerase transcribed genes (tRNA's) are enhanced by positioning of nucleosomes at the promoter regions (Shivaswamy et al., 2006). Positioning of nucleosomes at the promoter of these 
General introduction

regions brings distally placed promoter elements and transcriptional start sites within close proximity

\section{$\underline{1.5 \text { Chromatin remodelling }}$}

Chromatin structure has a requirement to be dynamic, as an unalterable chromatin state would not allow for differential transcriptional activity. This dynamic nature is achieved through chromatin remodelling. Remodelling of chromatin refers to the movement of nucleosomes to bring about a change in chromatin structure. This may occur through many mechanisms, for example, changes in ionic concentration, salt concentration and thermal energy required to induce nucleosome movement both short range and over longer distances (Marky and Manning, 1995). Remodelling complexes possess nucleosome re-positioning abilities and their action can result in de/condensation of chromatin.

\subsubsection{Translational and rotational nucleosome positioning}

There are two types of nucleosome positioning in relation to DNA sequence: translational and rotational. Translational nucleosome positioning refers to the linear region of DNA that the nucleosome occupies. There does not appear to be a consensus sequence for strong translational nucleosome positioning. However, DNA sequence patterns, such as the $[\mathrm{AA} / \mathrm{TT}]$ dinucleotide repeat, confer a higher statistical probability with which a histone octamer selects a contiguous stretch of 147 base pairs DNA (Thalstrom et al., 2004). These sequence patterns could constitute likely signals which represent preferred local sequences involved in direct DNA protein interaction. Translational positioning is more likely to occur as a result of ATP-dependant chromatin remodelling of a rotationally positioned nucleosome away from the energetically favourable DNA structure.

Rotational positioning refers to the orientation in which the DNA molecule is positioned as it wraps around the histone octamer with respect to the parts of the molecule that are facing towards the octamer. This has implications for binding of regulatory elements, such as promoter elements, to the DNA molecule as part of the molecule is 'protected' by the histone proteins and part is exposed. Rotational 
General introduction

positioning signals give permanent directional curvature to the DNA and have been shown to occur over TATA - containing promoters. Therefore, they also act as translational signals, as a position with a strong rotational preference (or curvature) is more likely to have a nucleosome occupying it. The $\beta$-phaseolin gene in tobacco is active during embryogenesis, but transcriptionally silent at all other developmental stages. The silenced state is consistent with a rotationally-positioned nucleosome over three phased TATA boxes within the promoter (Li et al., 1998). The promoter nucleosome is removed by chromatin remodelling during seed development, when the gene becomes transcriptionally active.

Two classifications of nucleosome movement exist, sliding and translocation. Sliding refers to the movement of a nucleosome along a DNA molecule, while translocation refers to the removal of the histone octamer to another DNA molecule (Lorch et al., 2004).

\subsubsection{ATP dependant remodelling complexes}

The ATP-dependent remodelling complex SWI/SNF was discovered in Sachharomyces cerevisiae. This discovery arose from a series of genetic studies, which were intending to identify functions of specific genes (Winston and Carlson, 1992, Hsieh and Fischer, 2005). These included (among others) the HO endonuclease gene (involved in mate-type switching) and the $S U C$ invertase gene (which is required for hydrolysis of glucose to raffinose). The analyses resulted in the discovery of a set of overlapping regulatory genes and were named SWI/SNF (SWI- switch and SNF- sucrose non-fermenter). These have since been shown to act as transcriptional regulators for many genes and are evolutionarily conserved across eukaryotes (Vignali et al., 2000).

The SWI/SNF remodelling complexes are divided into several classes. These are characterised by the proteins, other than the ATPase, found in the complex. The different classes exhibit different mechanisms and vary in their targets and actions. They are abundant in the genome, with over 40 present in Arabidopsis. 
The SWI2/SNF2 class has a conserved ATPase domain, a bromodomain in Cterminal, as well as two other conserved domains of unknown function (Vignali et al., 2000). These complexes are mostly involved in alteration of the winding of the DNA molecule around a histone octamer, and therefore are mostly involved in transcriptional activation and repression. The SWI2/SNF2 group include:

- $\mathrm{SWI} / \mathrm{SNF}$ in yeast (ySWI/SNF)

- Brahma complexes isolated from Drosophila

- Human BRM (hBRM)

- BRG1 complexes (mouse homologue mBRG1)

ISWI (Imitation SWItch) also, has a conserved ATPase domain, but is smaller than the SWI2/SNF2 class, having fewer subunits and shows homology to the SWI2/SNF2 proteins only over the ATPase domain. They require the presence of Histone $\mathrm{H} 4 \mathrm{~N}$-terminal tail for full activation (they are activated by DNA in nucleosomes). ISWI action includes relocation of nucleosomes by sliding along DNA and is mostly involved with maintaining higher order structure and chromatin assembly (Längst and Becker, 2001). The ISWI group include:

- ATP-utilising chromatin assembly and remodelling factor (ACF)

- Nucleosome remodelling factor (NURF)

- Chromatin assembly complex (CHRAC)

\subsubsection{Mode of action, twist defect and loop recapture}

Two models for the mechanism of the movement of a nucleosome along the DNA molecule have been suggested. These are the twist defect model and the loop recapture model (Fig 1.4) (Längst and Becker, 2004). The twist defect model relies on local alterations of DNA torsion around the nucleosome, brought about by the 


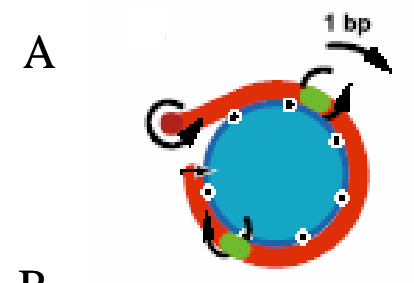

B

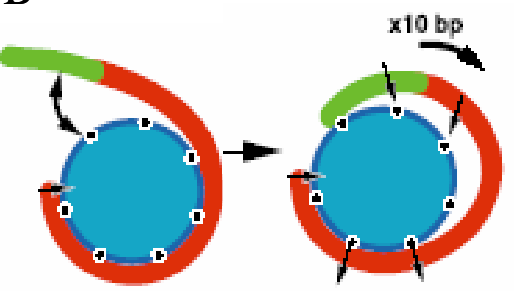

Figure 1.4 Schematic diagram to show the possible remodelling mechanisms. A: twist defect and B: loop recapture models Image from (Langst et al,. 1997)

action of remodelling complexes. These changes in direction twist the DNA away from the surface of the nucleosome, and therefore change the rotational position. The loop recapture model suggests that the DNA molecule detaches at a specific point. More of the DNA molecule is incorporated into the nucleosome while still in contact with the histone octamer, creating a loop or bulge. This loop is propagated along the length of the nucleosome DNA by subsequent detachment in front of the loop, and reattachment of the DNA molecule and the octamer behind the bulge. This results in a stepwise movement of the nucleosome along the DNA molecule to an alternate position (Flaus and Owen-Hughes, 2004). There is evidence to show that the loop recapture model is likely to be the prevalent mechanism in vivo (Strohner et al., 2005, Lorch et al., 2004). 


\section{$\underline{1.6 \text { Variation in linker length and higher-order chromatin structure }}$}

Linker DNA refers to the DNA between nucleosome core particles and can exist as either naked or linker histone-bound. It has been described as being between 10 and $90 \mathrm{nt}$ in length (Robinson et al., 2006a) and thought to comprise of sequences which favour a straight or rigid structure, such as poly [A] tracts (Marx et al., 2006). The linker histone 'anchors' nucleosomes in place by attachment of one end of the protein to the linker DNA and the other end to the DNA within the nucleosome (Bustin et al., 2005). Variations in linker length have been observed between and within species. Estimations of linker length were collected from the literature and published as a species comparative list (van Holde, 1979). Fourier analyses of this collection of linker lengths revealed a $10 \mathrm{bp}$ periodicity for linker lengths, which is thought to reflect the rotational positioning of nucleosomes on $10 \mathrm{bp}$ sequence repeats (Widom, 1992). A similar periodicity was reported in Human DNA (Kato et al., 2003). However these linker lengths were derived from nucleosome positions predicted by the DNA sequence patterns, while the periodicity was not found in the distribution of lengths of $>1000$ dinucleosome fragments isolated from K625 cells.

Variation in linker length is thought to be related to the level of transcriptional activity of the cell from where the chromatin originated, with shorter linker lengths and more tightly packed nucleosomes indicated areas of lower transcriptional activity (Olins et al., 1976). An association between linker length and chromatin fibre diameter was established by electron microscopy measurements of chromatin from three tissues with different nucleosome repeat lengths (NRL, the length from the start of one nucleosome to the start of the next): chicken erythrocyte (NRL $=208 \mathrm{bp}$ ), mouse thymus $(\mathrm{NRL}=195 \mathrm{bp})$ and sea cucumber spermatozoa $(\mathrm{NRL}=277 \mathrm{bp})$. While the level of compaction, and therefore the chromatin fibre diameters varied with salt concentrations, the size of the fibres consistently reflected the NRL s of the chromatin from which they were formed (Alegre and Subirana, 1989).

To understand the higher-order folding of chromatin, experiments involving measurements under different conditions have been performed. This revealed that compaction is dependent on salt concentration. Nucleosomes containing linker histones in low salt adopt a zig-zag conformation, while in the absence of $\mathrm{H} 1$, the 
General introduction

'beads on a string' structure is observed (Sun et al., 2005). In high salt concentrations (10 $\mathrm{mM}$ monovalent) the trajectory of the DNA as it exists the nucleosome is altered and forms a 'stem structure'(Woodcock and Horowitz 1997).

Two models for chromatin compaction beyond the nucleosome level exist: the solenoid and the zig-zag. The solenoid structure (also called one-start helix structure) was originally suggested by Finch and Klug (1976). In this model, the nucleosomes are packed adjacent to one another, with the linker DNA bent between nucleosomes to accommodate placement of the neighbouring nucleosome continuing the coil established by the nucleosome. Therefore, in the solenoid model the $30 \mathrm{~nm}$ fibre diameter is not affected by the length of the linker DNA. In the zig-zag model (also known as crossed linker and 2-start helix structure models) the linker crosses the middle of the $30 \mathrm{~nm}$ fibre, and neighbouring nucleosomes sit opposite each other in the $30 \mathrm{~nm}$ fibre (Wong et al., 2007). A zig-zag model arrangement of nucleosomes would favour either straight or flexible DNA but not likely to favour curved DNA. The resulting chromatin fibre would be affected by changes in linker length, however the ability of DNA to stretch around the histone octamer allows for discrepancies in linker length of up to $4 \mathrm{bp}$ without affecting higher order structure (Davey et al., 2002).

(a)

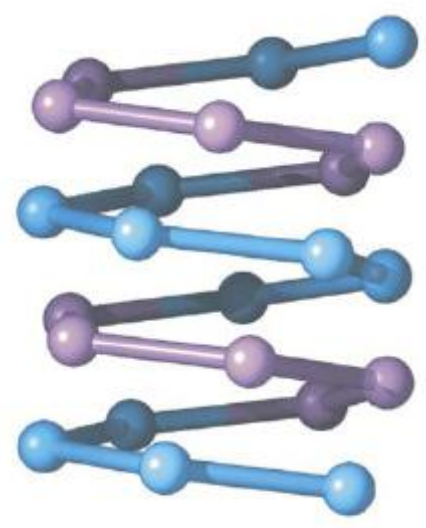

(b)

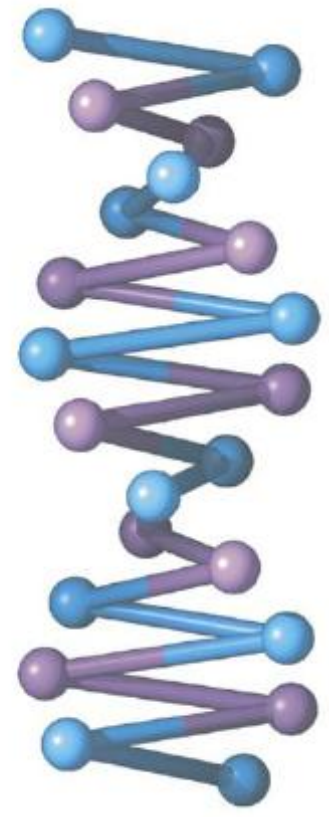

Figure 1.5 Schematic representations of the different models of chromatin fibre folding, A: the solenoid (or 1-start) model and B: the zig-zag (or 2-start) model. Image from Robinson et al., (2006b). 
General introduction

Recent work in this area suggests that a non-linear relationship exists between linker length and chromatin fibre diameter (Robinson et al., 2006a). The strong nucleosome-positioning DNA sequence '601' was used to reconstitute nucleosome arrays of up to 72 nucleosomes, with evenly spaced nucleosomes and linkers ranging from 10 to $70 \mathrm{bp}$. Measurements of chromatin fibres showed two classes (1) arrays with NRL from177-207 had an average diameter of $33 \mathrm{~nm}$ and 10.8 nucleosomes/11 $\mathrm{nm}$ and (2) arrays with NRLs of 217-237 bp had a diameter of 43-ish nm and 14-15 nucleosomes $/ 11 \mathrm{~nm}$. They suggested a model where the preferred structure of the chromatin fibre is a left-handed one-start helix (solenoid) and that the linker histone contributes to bending intrinsically straight DNA into the correct trajectory.

This work has been further enhanced by computer modelling of linker trajectory and the organisation of nucleosomes within chromatin fibre, for increasing linker lengths. These models suggest a different structure of fibre for each $10 \mathrm{bp}$ 'step' in linker length, and also suggests that both solenoid-type and zig-zag type structure may be present (Wong et al., 2007). 


\subsection{Recent advances and tools for chromatin studies.}

Epigenetic studies are benefiting from recent advances in microarray and sequencing technologies. Previous studies in Arabidopsis had identified methylated regions of heterochromatin on chromosome 4 (Lippman et al., 2004) at $1 \mathrm{~kb}$ resolution. However, global profiling of Arabidopsis DNA methylation sites using the Affymetrix GeneChip ${ }^{\circledR}$ Arabidopsis1.0R tiling array, has revealed patterns of DNA methylation specific to particular gene classes (Zhang et al., 2006). Of all expressed genes, $61.5 \%$ were found to be un-methylated and this group was enriched for transcription factors; $33.3 \%$ were methylated in the body of genes, which tended to be constitutively expressed genes; and $5.2 \%$ genes were methylated in the promoter. Promoter methylated genes were more likely to be expressed in a tissue-specific manner. The Arabidopsis methylome has been even more accurately mapped to base pair resolution by Solexa sequencing of bisulphite-treated DNA (Lister et al., 2008).

The tiling microarray approach was used to identify the translational positions of 2278 nucleosomes in Saccharomyces cerevisiae (Yuan et al., 2005). Nucleosome DNA fragments were liberated by micrococcal nuclease digestion and hybridised to microarrays containing 50 bp probes tiled every 20 bp. Probes covered most of chromosome III and a further 223 genes on other chromosomes. These experiments revealed that $65-69 \%$ nucleosomes in yeast are well positioned. De-localised (or 'fuzzy') nucleosomes tend to be found at highly expressed genes, which suggests that nucleosomes are transiently disassembled and re-assembled, thus allowing for the passage of RNA polymerase. This was the first time that high-resolution microarrays had been used to study nucleosome positioning. Analysis of the yeast nucleosome landscape by tiling arrays revealed that $81 \%$ of the genome is occupied by nucleosomes (Lee et al., 2007). This experiment revealed that the intergenic regions are nucleosome-depleted compared with the coding regions. A feature which has now been shown in human chromatin (Schones et al., 2008). This experiment also revealed a pattern in nucleosome occupancy at the transcriptional start sites (TSS), where there appears to be higher occupancy to the right of the TSS (upstream).

Chromatin structure at human promoters has also been investigated using a tiling microarray. Mononucleosomal DNA was hybridised to microarrays containing 
General introduction

probes 50 nucleotides in length and staggered every 10 bp (Ozsolak et al., 2007). Microarray probes covered $1.5 \mathrm{~kb}$ promoter regions of 3692 genes. Seven cell lines were compared and a high degree $(90 \%)$ of reproducibility between experiments was observed. Approximately $88 \%$ of the promoters investigated were found to contain at least one positioned nucleosome, and expressed genes were more likely to have a nucleosome-depleted region of approximately $100 \mathrm{bp}$ spanning the transcriptional start site. It was suggested that this approach could be used as a tool for the analysis of chromatin structure in disease, as the microarray contained promoter regions of all the genes represented on the Affymetrix Human Cancer Array.

The increase in available nucleosome positioning data will aid the refinement of nucleosome prediction models. A novel prediction algorithm has been developed, based on nucleosome positioning sequence preferences identified from 199 experimentally determined yeast nucleosome DNA fragments (Segal et al., 2006). The algorithm was designed to recognise $[\mathrm{AA}],[\mathrm{TT}]$ and [TA] dinucleotides with a $10 \mathrm{bp}$ periodicity, and was able to predict approximately $50 \%$ of nucleosome positions experimentally determined using a tiling microarray (Yuan et al., 2005). The nucleosome positions identified using the microarray were also compared to computationally identified nucleosome positioning signals for gene regions $(-1,000$ bp to +800 bp relative to the transcriptional start site) (Ioshikhes et al., 2006). These analyses revealed high degree of accuracy in predicting nucleosome positions at yeast promoters. These models have since been superseded by the more recent probabilistic model developed by the Segal lab (Kaplan et al., 2008). This was derived from yeast in vitro positioning data and scores the nucleosome formation potential. This model therefore represents only nucleosome sequence preferences.

Massively parallel sequencing technologies have been utilised to map the positions of nucleosomes containing the histone variant H2A.Z in Saccharomyces cerevisiae cells (Albert et al., 2007). While some nucleosomes are always strongly positioned, many exhibit some cell to cell translational variation. This technology allows a more accurate analysis of nucleosome position than tiling microarrays, as multiple sequence reads are obtained for translational nucleosome positions. A map of H2A.Z has also been constructed for Arabidopsis, which revealed an interesting 
General introduction

relationship between this histone variant and DNA methylation (Zilberman et al., 2008). H2A.Z-containing nucleosomes appear to protect the DNA from DNA methylation within euchromatic regions, conversely, the H2A.Z -containing nucleosomes are excluded from heavily methylated areas such as the pericentromeric repeats. High-throughput sequencing of the yeast and Drosophila nucleosomes has revealed some interesting patterns of occupancy around the TSS (Mavrich et al, 2008a; Mavrich et al.,2008b). Both genomes show a peak in nucleosome occupancy after the TSS, and ordered nucleosome phasing thereafter (within the gene region). However the distance of the peak was different for the two organisms resulting in the TSS being nucleosome-free in Drosophila, but the TSS was buried within the nucleosome in yeast. This suggests that there may be different mechanisms regulating transcriptional control in these two species.

Recent advances in high-throughput technologies have allowed the study of genomewide locations of histone modifications and variants in Arabidopsis. The trimethylation of histone $\mathrm{H} 3$ at lysine 27 is established and maintained by $\mathrm{PcG}$ proteins and is involved in silencing more than 4,000 Arabidopsis genes (Zhang et al., 2007). Conversely, the histone modifications $\mathrm{H} 3 \mathrm{~K}_{4} \mathrm{me}^{3}$ and $\mathrm{H} 3 \mathrm{~K} 9 \mathrm{ac}$ are associated with the transcriptionally active genes involved in drought stress in Arabidopsis (Kim et al., 2008). 


\section{$\underline{1.8 \text { Nucleosome positioning databases }}$}

A publicly available set of nucleosomal sites and sequences has been available in a nucleosome position region database (NPRD) (Levitsky et al., 2005). The database contains a compilation of experimentally determined nucleosome positions. Each entry relates to an individual nucleosome site, and details associated with that site, for example, the relationship between nucleosome position and type of gene activity, type of nucleosome position (rotational or translational) and factors influencing nucleosome position. The NPRD contains sequences representing a variety of eukaryotic and viral nucleosome sites over a variety of genomic regions (Figure 1.6). However, only 2 plant-derived data are represented (Zea mays). The current version of the database contains 112 entries (February 2007) and is still in development (112 entries, last checked 01/09/09).

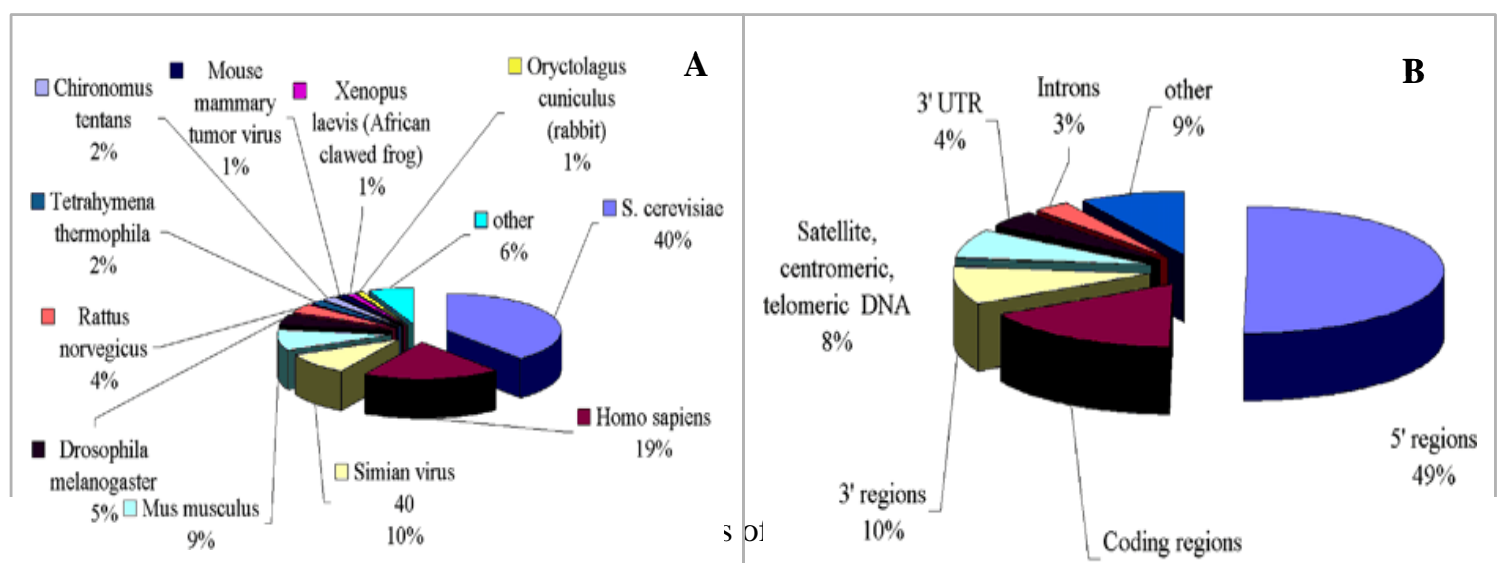

Figure 1.6 A Chart showing the relative numbers of organisms represented in the NRPD (2005 version). B Shows a representation of genomic regions represented in the database. Images from Levitsky et al., (2005) 


\subsection{Project aims}

The motivation for this project arose from a lack of plant-derived nucleosome positioning data, and the need to understand the regulation of plant gene expression at the chromatin level. At the beginning of this project, there were plant-derived nucleosome positioning data for only a few individual genes, but no global studies had been carried out.

\subsubsection{Scientific Hypotheses to be tested}

1. Arabidopsis nucleosome sequence composition is different from the genome average and there are differences in nucleosome and linker DNA.

2. Arabidopsis nucleosome sequences display specific distributions in their dinucleotide complement which are known to contribute to the bending of DNA around the nucleosome and that these preferences are similar to those discovered in other taxa

3. The distribution of linker length in Arabidopsis is comparable to that determined for other taxa and Periodicity exists within the Arabidopsis linker length distribution.

4. Features of the observed distribution of Arabidopsis linker length show similarities to the distribution of Arabidopsis intron length, which may indicate a relationship between introns in the DNA and chromatin structure.

5. Nucleosomes are evenly distributed throughout the Arabidopsis genome, and biases in nucleosome occupancy differ between coding and non-coding regions in Arabidopsis.

6. Ordered positioning of nucleosomes exists around the TSSs in Arabidopsis, comparable to the organisation found within other taxa.

7. The Arabidopsis Nucleolar Organiser Regions display different nucleosome patterns of positioning dependent on chromosomal origin, due to the transcriptionally heterogeneous nature of these regions. 
General introduction

8. Overall differences in nucleosome positioning exist between wild-type plants and plants with depleted ${ }^{5 \mathrm{~m}} \mathrm{C}$ levels.

\subsubsection{Experimental approaches}

To address the above hypotheses, the following approaches were taken:

Dinucleosome libraries have been constructed from A. thaliana tissue. A subset of clones has been sampled to produce a set of $~ 500$ dinucleosomal sequences. These sequences were used to determine linker length distribution and analysed for sequence patterns.

High-throughput sequencing technologies (Roche 454 FLX and Solexa) were used to determine the sequence and genomic positions of mononucleosomes and dinucleosomes throughout the Arabidopsis genome. Specific-dinucleotide and linker length distributions were calculated from DNA fragment data and nucleosome occupancy within specific gene classes and at the transcriptional start sites were investigated.

Mononucleosome DNA was isolated from the Arabidopsis METl-antisense to determine any differences in the sequences composition, nucleosome spacing and genomic occupancy of nucleosomes in a DNA methylation-deficient environment. 
Chapter 2

General materials and methods 


\subsection{General laboratory protocols}

All reagents from Sigma unless otherwise stated.

\section{1.i Plant material}

Arabidopsis thaliana Columbia-0 (Col-0) and antisense MET1 line 10.1, T4 generation (C24 ecotype) (Finnegan et al., 1996) were sterilised in $25 \%$ Parazone for five minutes, washed with $70 \%$ ethanol for two minutes, rinsed twice and resuspended in sterile water. Seeds were cold-treated at $4{ }^{\circ} \mathrm{C}$ for five days and sown onto sterile half-strength Murashige and Skoog media (M0404) with Gamborgs vitamins, containing $1 \%$ sucrose and $1 \%$ agar (Bacto), $\mathrm{pH}$ 5.7. Plates were incubated at $18-22^{\circ} \mathrm{C}$ in constant light for 7 days. Seedlings were transferred to soil (Levington F2's and intercept 5GR $280 \mathrm{~g} / \mathrm{m}^{3}$ ) and grown in long day conditions (16 hours light $300 \mu \mathrm{mol} \mathrm{m}-2$ sec-1 from Osram HQI-BT 400W/D metal halide lamps at $23^{\circ} \mathrm{C}$ day $65 \%$ relative humidity $/ 8$ hours dark, $18^{\circ} \mathrm{C} 70 \%$ relative humidity). Leaf material (rosette leaves 6, 7 and 8) was harvested 18-21 days post germination and cross-linked immediately following harvest (Section 2.1.ii).

\section{1.ii Formaldehyde cross-linking of protein and DNA}

The cross-linking procedure was carried out in a fume hood. Formaldehyde crosslinking of protein and DNA was adapted from the method of Gendrel et al., (2005). The fresh Arabidopsis leaf material was added to $37 \mathrm{ml}$ of $1 \%$ formaldehyde. The leaf/formaldehyde mixture was vacuum-infiltrated at room temperature for 15 minutes (Heto, Sue-300). To stop the cross-linking reaction from progressing any further, $2.5 \mathrm{ml}$ of $2 \mathrm{M}$ glycine (to final concentration of $0.125 \mathrm{M}$ ) was added to the leaf tissue and was vacuum-infiltrated for an additional 5 minutes. The tissue was rinsed with sterile water to remove the formaldehyde and blotted dry between paper towels. Nuclei were isolated from cross-linked tissue immediately following the cross-linking step. 


\section{1.iii Nuclei isolation}

The nuclei isolation protocol was adapted from the method of Bowler et al., (2004). Fresh leaf tissue (20 g) was ground in $30 \mathrm{ml}$ pre-cooled Nuclei extraction buffer, filtered through two layers of miracloth (Calbiochem, cat.\# 475855) into cold $50 \mathrm{ml}$ sterile centrifuge tubes (TPP). The extract was centrifuged at $2500 \mathrm{x} \mathrm{g}$ for 10 minutes at $4^{\circ} \mathrm{C}$ using a bench top centrifuge (Sigma 3K10). The supernatant was discarded and the extract washed three times by re-suspension in $50 \mathrm{ml}$ ice-cold nuclei wash buffer and centrifuged at $3000 \mathrm{x}$ g for six minutes. Following the final wash, the supernatant was discarded and the pellet was re-suspended in $1 \mathrm{ml}$ nuclei wash buffer. Nuclei were layered over an ice-cold 1.5 M sucrose (Fisher) solution in a sterile centrifuge tube and centrifuged for three minutes, $3000 \mathrm{xg}$ at $4^{\circ} \mathrm{C}$ to remove remaining chloroplasts. The supernatant was removed and the nuclei pellet was resuspended in $4 \mathrm{x}$ pellet volume in nuclei storage buffer and an equal volume of glycerol was added. The suspension was divided into two aliquots, the first containing $25 \%$ of the total volume of the nuclei suspension, the other containing $75 \%$. Nuclei were frozen in liquid nitrogen and stored at $-80{ }^{\circ} \mathrm{C}$.

Nuclei isolation buffer:

1 M Hexalene glycol (Fisher Scientific)

10 mM PIPES/KOH pH 7.0 (Fluka)

$10 \mathrm{mM} \mathrm{MgCl}_{2}$ (Fluka)

$0.2 \%$ Triton X-100 (Promega)

$5 \mathrm{mM}$ 2-mercaptoethanol

$1 \mathrm{mM}$ AEBSF (4-(2-Aminoethyl)benzenesulfonyl fluoride hydrochloride $(\mathrm{A} 8456))$

$\underline{\text { Nuclei wash buffer }}$

as isolation buffer, except $0.5 \mathrm{M}$ hexylene glycol

$\underline{\text { Nuclei storage buffer }}$

50 mM Tris-HCl, pH 7.5

$5 \mathrm{mM} \mathrm{MgCl}_{2}$ (Fluka)

0.1 mM EDTA (Duchefa) 
General materials and methods

\title{
2.1.iv Preparation of nucleosomal DNA
}

In order to determine the optimal concentration of micrococcal nuclease (MNase, Worthington Biochemical Corporation, USA) enzyme to produce nucleosome DNA fragments of the desired length, a small analytical digest was performed on a quarter of the nuclei preparation. This was performed for each new batch of chromatin in nuclei prepared.

\section{1.v Preparation of the enzyme}

MNase was re-suspended in MNase buffer at a concentration of $15 \mathrm{U} / \mu \mathrm{l}$ and $50 \mu \mathrm{l}$ aliquots were stored at $-20^{\circ} \mathrm{C}$ prior to use.

\author{
$\underline{\text { MNase buffer }}$ \\ $10 \mathrm{mM}$ Tris- $\mathrm{HCl} \mathrm{pH} 7.5$ \\ $0.5 \mathrm{mM}$ EDTA \\ $0.5 \mathrm{mM}$ DTT
}

\section{1.vi Quantitative Micrococcal Nuclease digests}

The optimal concentration of MNase required to digest partially chromatin in nuclei was determined by digestion of $25 \%$ of the total nuclei preparation. Nuclei were thawed on ice, pelleted by centrifugation at $3000 \mathrm{x} \mathrm{g}$ for three minutes and resuspended in $450 \mu \mathrm{l}$ of buffer M. Aliquots of $150 \mu \mathrm{l}$ were transferred to $3,1.5 \mathrm{ml}$ microfuge tubes. Chromatin in nuclei was digested with 1,10 and $50 \mu$ aliquots of a $1 / 500$ dilution MNase $(1 / 500=0.03 \mathrm{U} / \mu \mathrm{l})$ for 4 minutes at $37^{\circ} \mathrm{C}$. The reaction was stopped by the addition of $15 \mu \mathrm{l} 0.5 \mathrm{M}$ EDTA and DNA extracted.

\section{$\underline{\text { Buffer M }}$}

50 mM Tris- $\mathrm{HCl}$ pH 7.4

$60 \mathrm{mM} \mathrm{KCl}$ (Riedol-de Hean)

$3 \mathrm{mM} \mathrm{CaCl}_{2}$ (Fisher Scientific)

$50 \%$ glycerol (Fisher Scientific)

$0.34 \mathrm{M}$ sucrose (Fisher Scientific) 
To isolate nucleosomal DNA fragments, nuclei were transferred to a $2 \mathrm{ml}$ tube and DNA extraction buffer was added to a final volume of $1 \mathrm{ml}$. The nuclei were incubated at $55^{\circ} \mathrm{C}$ for 30 minutes. DNA was extracted using 24:1 chloroform:isoamyl alcohol, precipitated with ice-cold $100 \%$ ethanol, washed with ice-cold $70 \%$ ethanol and dried in a vacuum drier for 3-5 minutes. The DNA pellet was re-suspended in $20 \mu \mathrm{l} 1 \mathrm{x}$ TE buffer $(\mathrm{pH} 8)$ and treated with $10 \mu \mathrm{g}$ RNase A/T mix (Fermentas) at $37^{\circ} \mathrm{C}$ for 30 minutes. Formaldehyde cross-links were reversed by incubation at $65^{\circ} \mathrm{C}$ for 2 hours. DNA fragments were separated by electrophoresis on a $1.5 \%$ agarose (Bioline)/TBE gel containing Ethidium Bromide (Bio-Rad) (1\% vol), at $100 \mathrm{~V}$ for 40 minutes. Fragments were viewed using Bio-Rad Quantity One UV geldoc system.

\author{
DNA extraction buffer \\ $100 \mathrm{mM}$ Tris-HCl pH 8 \\ 50 mM EDTA pH 8 \\ $150 \mathrm{mM} \mathrm{NaCl}$ (Fisher Scientific) \\ $1 \%$ SDS \\ $100 \mu \mathrm{g} / \mathrm{ml}$ proteinase $\mathrm{K}$
}

\title{
2.1.vii Preparative Micrococcal nuclease digests
}

The remaining nuclei preparation was gently thawed on ice, pelleted and resuspended in $\mathrm{M}$ buffer, to twice the pellet volume. The suspension was digested with the optimal concentration of enzyme determined in the serial digest. Following extraction, DNA was re-suspended in $40 \mu \mathrm{l}$ sterile water, quantified (UV Nanodrop) and separated on an agarose gel (as above).

\section{1.viii Cloning}

DNA bands of approximately 300-400 bp were excised and purified using the Promega 'Wizard DNA gel purification and Clean-Up kit' according to the manufacturer's instructions. Fragments were cloned using the EcoRV site of 
General materials and methods

pSTBlue cloning vector ('Perfectly Blunt Cloning Kit '(Novagen)) following the manufacture's instructions, with the ligation reaction time increased to 25 minutes. Colonies were grown statically overnight at $37^{\circ} \mathrm{C}$ on $2 \times Y T$ containing $1 \%$ Agar (Bacto ${ }^{\mathrm{TM}}$ ), $50 \mu \mathrm{g} / \mathrm{ml}$ carbenicillin (Melford Laboratories), $12.5 \mu \mathrm{g} / \mathrm{ml}$ tetracycline (Duchefa), $70 \mu \mathrm{g} / \mathrm{ml} \mathrm{X-gal} \mathrm{(Melford} \mathrm{Laboratories),} \mathrm{and} 80 \mu \mathrm{M}$ IPTG (Melford Laboratories),

\section{$\underline{\text { Bacterial strain }}$}

Novablue Singles ${ }^{\mathrm{TM}}$ high-efficiency competent cells (Merck)

Genotype:

endA1 hsdR17 (rK12- mK12+) supE44 thi-1 recAl gyrA96 relAl lac $F^{\prime}[$ proA $+B+$ lacIqZDM15::Tn10] (TetR)

Colonies were screened for the presence of inserts by colony PCR using vectorspecific primers:

T7 promoter (F) 5'-TCTAATACGACTCACTATAGGG-3', U19-mer (R) 5'-GTTTTCCCAGTCACGACGT-3'.

Amplification conditions were: $94^{\circ} \mathrm{C} / 4$ minutes, $30 \times\left(94^{\circ} \mathrm{C} / 30\right.$ seconds, $52^{\circ} \mathrm{C} / 30$ seconds, $72^{\circ} \mathrm{C} / 1$ minute), $72^{\circ} \mathrm{C} / 5$ minutes and hold at $10^{\circ} \mathrm{C}$ ).

Colonies containing inserts were transferred to 96 deep-well $(1.2 \mathrm{ml})$ plates containing $200 \mu \mathrm{l} \mathrm{YT,} 10 \%$ glycerol and $50 \mu \mathrm{l} / \mathrm{ml}$ carbenicillin. Cultures were grown statically at $37^{\circ} \mathrm{C}$ for 12 hours and stored at $-80^{\circ} \mathrm{C}$. Plasmid DNA was amplified by from bacterial colonies by rolling circle amplification using the Templiphi $^{\mathrm{TM}}$ amplification kit (GE Healthcare). Bacterial cells were transferred into a $0.2 \mathrm{ml}$ PCR tube using a cocktail stick and a $1.25 \mu \mathrm{l}$ sample buffer was added. Cells were lysed for 5 minutes at $95^{\circ} \mathrm{C}$. A mixture of $1.25 \mu \mathrm{l}$ enzyme buffer and 0.05 $\mu l$ enzyme mix (containing $\$ 29$ polymerase and random hexamer primers) for each sample was prepared, and $1.25 \mu \mathrm{l}$ was added to each tube of cooled lysed cells. The reaction continued overnight at $30^{\circ} \mathrm{C}$, and was stopped by incubation for 10 minutes at $65^{\circ} \mathrm{C}$. 
General materials and methods

\section{1.ix Determination of clone library quality}

To check quality of library, inserts were sequenced directly from plasmids using Big Dye terminator kit V.31 (Roche) according to the manufacturer's instructions, using one-eighth of the Big Dye reaction mix recommended. Amplification conditions were: $96^{\circ} \mathrm{C} / 1.5$ minutes, $35 \mathrm{x}\left(96^{\circ} \mathrm{C} / 10\right.$ seconds, $50^{\circ} \mathrm{C} / 5$ seconds, $60^{\circ} \mathrm{C} / 4$ minutes $)$ and $22^{\circ} \mathrm{C}$ hold). Test reactions were sequenced at Oxford sequencing services.

\section{1.x Sequencing of clone library}

Plasmids containing inserts were amplified by the Templiphi ${ }^{\mathrm{TM}}$ rolling circle amplification (as above) in 96-well PCR plates. Plasmid DNA from the clone library was quantified and sequenced at the QIAGEN Genomic and Sequencing Services facility.

\section{1.xi Large scale sequencing of Arabidopsis nucleosome fragments}

Dinucleosome DNA fragments were prepared as described above (2.1.vii.) for sequencing by Roche 454 FLX. Library preparation of Arabidopsis Col-0 dinucleosome fragments by Roche 454 FLX was carried at the John Innes Genome Centre, Norwich, and sequencing was carried out at Cogenics (Hope end, Takeley, Essex, CM22 6TA) via the John Innes Genome Centre, Norwich.

Dinucleosome and mononucleosome fragments were prepared as described above (2.1.vii.) from wild-type and $M E T 1$ antisense plants. In order for the samples to be mixed and sequenced using a single solexa channel, a different 4 bp 'barcode/tag' was ligated to the ends of the fragments from each sample. This enabled the separation of sequence data into the two samples following sequencing. The barcode 'tags' were removed from the sequences prior to the data being released from GATC. Library preparation (including barcode and adapter ligations) and sequencing were carried out at GATC Biotech AG (Jakob-Stadler-Platz 7, D-78467 Konstanz, Germany). 
General materials and methods

\section{1.xii Hybridisation to the Affymetrix GeneChip ${ }^{\circledR}$ Arabidopsis Tiling 1.0R Array}

The Affymetrix GeneChip ${ }^{\circledR}$ Arabidopsis Tiling 1.0R Array was used to investigate genomic patterns of nucleosome occupancy. The array consists of $\sim 3.2$ million probe pairs (perfect-match and mis-match) covering the whole non-repetitive Arabidopsis genome, on a single chip. The probe sequences correspond to the reverse DNA strand and are $25 \mathrm{bp}$ in length. They are tiled with an average spacing of $10 \mathrm{bp}$ apart, resulting in an average resolution of $35 \mathrm{bp}$.

Mononucleosome DNA fragments were labelled with biotin and hybridised to the array. Labelling and hybridisations were carried out at the Nottingham A. thaliana Stock Centre (NASC) Affymetrix service. 
General materials and methods

\subsection{General data analysis protocols}

\section{2.i Sequence BLAST alignment}

Nucleosome DNA fragments were aligned to the Arabidopsis Col-0 reference genome (TAIR 8) using the Time-Logic Tera-probe BLAST (Smith-Waterman algorithm) alignment on a DeCypher server. Matches with $97 \%$ or greater similarity (for the clone fragments), or 100\% similarity (for the 454 FLX and Solexa DNA fragments) to the reference genome were used in the final datasets. The Tera-probe alignments were carried out by Graham King, Rothamsted Research.

\section{2.ii Paired-end matching}

The Tera-probe BLAST alignments output was parsed using the programme [Blastparser2.exe] (written in Delphi 7 by Graham King, Rothamsted Research); the output for each end was then imported into separate 'hits' tables in a MySQL database. Assignment of genomic co-ordinates for paired end sequences was obtained via database queries, using the following criteria: where the alignments in each pair were within $620 \mathrm{bp}$ and where the alignments in each pair were on opposite genomic DNA strands. The 5' co-ordinates of the two ends on opposite strands were output to a .CSV file, along with the chromosome and fragment ID. The sequence alignments were sorted into two groups for each dataset: all alignments (which include sequences that align to more than one genomic position with $100 \%$ similarity) and unique alignments (which exclude sequences which align to the genome more than once). All sequences were used for analysis of sequence characteristics and linker length distributions, while unique alignments only were used in analyses of nucleosome position. Paired-end matching and sorting into unique and non-unique datasets was performed by Graham King, Rothamsted Research. The Delphi 7 scripts were executed, and the MySQL database was built and accessed using a PC.

\section{2.iii Extraction of nucleosome sequences}

Nucleosome sequences were extracted from the Col-0 reference Arabidopsis genome using a PERL script called [find_seq.pl] (Table 2.1). All PERL scripts were 
General materials and methods executed using a desktop PC and the program Perl Express 2.5 (freeware, url: http://www.softdll.com/phpaspperl/perlexpress.html). Sequences were trimmed into subsets of mononucleosome and linker, using the LEFT and RIGHT functions in Excel.

Table 2.1 A list of PERL scripts generated and used in this study.

\begin{tabular}{l|l}
\hline Perl script & Description \\
\hline find_seq.pl & $\begin{array}{l}\text { Extracts sequences from large sequence files using lists of co-ordinates. } \\
\text { Counts the end dinucleotide (terminal dinucleotide in position 1) and generates } \\
\text { a frequency table. } \\
\text { Counts each dinucleotide in a given sequence, over a moving window of two } \\
\text { (so each base is considered twice, the preceding and following one). } \\
\text { count_dinuc.pl }\end{array}$ \\
rand_seq.pl & $\begin{array}{l}\text { Uses the rand function of PERL to generate a series of random numbers within } \\
\text { a given size. }\end{array}$ \\
counts the numbers of A,C,G and T for each sequences in a sequence file \\
count_freqs.pl
\end{tabular}

\section{2.iv Detection of periodicity}

Periodicities in dinucleotide and linker length distribution were calculated using Fourier analysis, which is a standard method for detecting sequence periodicities (Satchwell et al., 1986). Distributions were once or twice-differenced to eliminated the overall trend in the data (twice-differencing was required in most cases). The periodicity was calculated using a programme written by Steve Powers (Biomathematics and Bioinformatics Dept.) Rothamsted Research, utilising the periodtest procedure in GenStat ${ }^{\circledR}\left(2008,11^{\text {th }}\right.$ Edition, VSN International, UK). Nonparametric $95 \%$ confidence limits for important peaks were calculated by the equation:

$\mathrm{CI}=0.1026 \times \sqrt{\frac{\left.\text { Variance }_{\text {(periodogram }}\right)}{2}}$

Periodicities were statistically significant where the peak was larger than the neighbouring peaks + the CL. 
General materials and methods

\section{2.v Kernel Density Estimation}

Kernel density estimation was used to smooth the nucleosome distributions in order to reveal long-term trends in the distribution. Kernel density estimates were calculated in Genstat v11, using the method of Sheather and Jones (1991), and 2048 grid points.

\section{2.vi Construction of nucleosome occupancy files}

To investigate nucleosome occupancy within different annotation regions, occupancy files were constructed. A gene annotation code was designed (Table 2.2) and assigned to each nucleotide position in the Arabidopsis genome in successive rounds, so that each new pass overwrites the previous annotation. Annotation codes were written in the following order: transcriptional units, translational units, 5' and 3' UTRs, exons and introns, 5' UTR exons and intron and 3' UTR exons and intron (Figure 2.1). For alternatively-spliced annotation, introns are given preference over exons since the introns are written after exons. The annotation code appears as a column in the nucleosome occupancy files alongside the nucleotide position.

Table 2.2 Annotation codes assigned to each bp position in Arabidopsis in construction of nucleosome occupancy files.

\begin{tabular}{l|llllllll}
\hline & miRNA & Misc & Protein & Pseudo- & rRNA & snoRNA & snRNA & tRNA \\
& mi & RNA & coding & gene & & & & no \\
\hline NS & NS_mi & NS_sc & NS_pc & NS_pg & NS_rr & NS_no & NS_sn & NS_tr \\
TU & TU_mi & TU_sc & TU_pc & TU_pg & TU_rr & TU_no & TU_sn & TU_tr \\
TR & TR_mi & TR_sc & TR_pc & TR_pg & TR_rr & TR_no & TR_sn & TR_tr \\
U5 & U5_mi & U5_sc & U5_pc & U5_pg & U5_rr & U5_no & U5_sn & U5_tr \\
U3 & U3_mi & U3_sc & U3_pc & U3_pg & U3_rr & U3_no & U3_sn & U3_tr \\
EX & EX_mi & EX_sc & EX_pc & EX_pg & EX_rr & EX_no & EX_sn & EX_tr \\
IN & IN_mi & IN_sc & IN_pc & IN_pg & IN_rr & IN_no & IN_sn & IN_tr \\
E5 & E5_mi & E5_sc & E5_pc & E5_pg & E5_rr & E5_no & E5_sn & E5_tr \\
I5 & I5_mi & I5_sc & I5_pc & I5_pg & I5_rr & I5_no & I5_sn & I5_tr \\
E3 & E3_mi & E3_sc & E3_pc & E3_pg & E3_rr & E3_no & E3_sn & E3_tr \\
I3 & I3_mi & I3_sc & I3_pc & I3_pg & I3_rr & I3_no & I3_sn & I3_tr \\
\hline
\end{tabular}


Example Arabidopsis sequence (gene model)

1, Assign nucleotide sequence (NS)

2, Assign transcriptional unit (TU)

3, Assign translation region (TA)

4, Assign 5' UTR (5U)

5, Assign 3' UTR (3U)

6, Assign exons (EX)

7, Assign introns (IN)

8, Assign 5'UTR exons (E5)

9, Assign 5'UTR introns (I5)

10, Assign 3’UTR exons (E3)

11, Assign 3'UTR introns (I3)

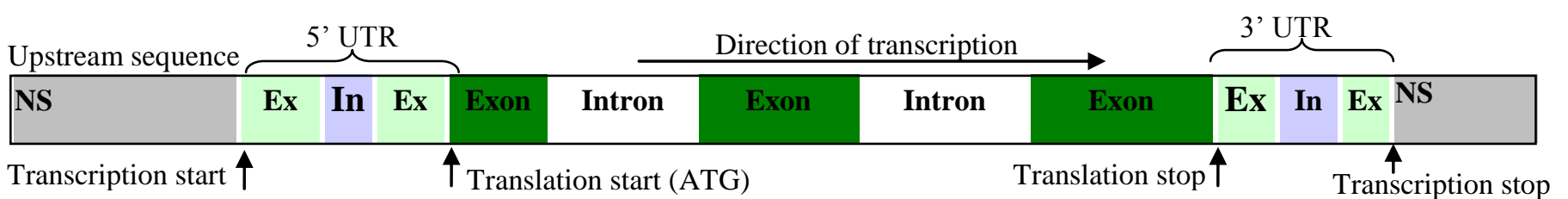

\begin{tabular}{|c|c|c|c|c|c|c|c|c|c|c|c|c|}
\hline NS & & & & & & & & & & & & NS \\
\hline NS & $\mathbf{T U}$ & & & & & & & & & & & NS \\
\hline NS & TU & & & TA & & & & & & & & NS \\
\hline NS & $5 \mathbf{U}$ & & & TA & & & & & & & & NS \\
\hline NS & $5 \mathrm{U}$ & & & TA & & & & & $3 \mathbf{U}$ & & & NS \\
\hline NS & $\mathrm{EX}$ & $5 U$ & & $\mathbf{E X}$ & TA & EX & TA & $\mathbf{E X}$ & & $3 \mathbf{U}$ & $\mathbf{E X}$ & NS \\
\hline NS & EX & IN & & EX & IN & EX & IN & EX & & IN & $\mathrm{EX}$ & NS \\
\hline NS & E5 & IN & E5 & EX & IN & EX & IN & EX & & IN & EX & NS \\
\hline NS & E5 & I5 & E5 & EX & IN & EX & IN & EX & & IN & EX & NS \\
\hline NS & E5 & I5 & E5 & EX & IN & EX & IN & $\mathbf{E X}$ & E3 & IN & E3 & NS \\
\hline NS & E5 & I5 & E5 & EX & IN & $\mathbf{E X}$ & IN & $\mathbf{E X}$ & E3 & I3 & E3 & NS \\
\hline
\end{tabular}

Figure 2.1 Schematic showing the order of assignment of gene annotation to each Arabidopsis nucleotide. The annotation is re-written in successive rounds until all annotations are added. 
General materials and methods Nucleosome occupancy was calculated by counting the number times each nucleotide position is represented within a nucleosome DNA fragment. Measures of nucleosome occupancy were added to a column in the nucleosome occupancy files.

To investigate the effect of DNA methylation on nucleosome positioning, previously published DNA methylation data (Lister et al., 2008) were downloaded from supplementary material from the science direct website (url:http://www.sciencedirect.com/science?_ob=ArticleURL\&_udi=B6WSN4S9G216-).

DNA methylation data was added to the nucleosome occupancy files in the same manner as the nucleosome occupancy. The nucleosome annotation files were constructed by Graham King, Rothamsted Research using a PC. 
General materials and methods

\section{2.vii Description of external datasets}

A description of the external (publicly-available) datasets used in this thesis are shown in Table 2.3.

Table 2.3 A description with references of the publicly available datasets used in this thesis.

\begin{tabular}{|c|c|c|c|c|c|}
\hline Dataset & Species & $\begin{array}{l}\text { Nucleosome } \\
\text { class }\end{array}$ & Technology & $\begin{array}{l}\text { Number of } \\
\text { entries }\end{array}$ & Reference \\
\hline List_wt_meth & Arabidopsis & ${ }^{5 \mathrm{~m}} \mathrm{C}$ & Bs-solexa & & $\begin{array}{l}\text { Lister et al, } \\
2008\end{array}$ \\
\hline $\begin{array}{l}\text { List_met- } \\
\text { 1_meth }\end{array}$ & Arabidopsis & ${ }^{5 \mathrm{~m}} \mathrm{C}$ & Bs-solexa & & $\begin{array}{l}\text { Lister et al, } \\
2008\end{array}$ \\
\hline $\begin{array}{l}\text { Johns_Celega } \\
\text { ns }\end{array}$ & C. elegans & mono & 454 & 327,294 & $\begin{array}{l}\text { Johnson et al., } \\
2006\end{array}$ \\
\hline $\begin{array}{l}\text { Trav_chick_di } \\
\text { nuct }\end{array}$ & Gallus & di (trim) & $\begin{array}{l}\text { Traditional } \\
\text { Sanger } \\
\text { sequencing }\end{array}$ & 49 & $\begin{array}{l}\text { Satchwell and } \\
\text { Travers } 1989\end{array}$ \\
\hline $\begin{array}{l}\text { Trav_chick_di } \\
\text { писи }\end{array}$ & Gallus & di (untrim) & $\begin{array}{l}\text { Traditional } \\
\text { Sanger } \\
\text { sequencing }\end{array}$ & 103 & $\begin{array}{l}\text { Satchwell and } \\
\text { Travers } 1989\end{array}$ \\
\hline $\begin{array}{l}\text { Trav_chick_m } \\
\text { onoc }\end{array}$ & Gallus & mono & $\begin{array}{l}\text { Traditional } \\
\text { Sanger } \\
\text { sequencing }\end{array}$ & 152 & $\begin{array}{l}\text { Satchwell and } \\
\text { Travers } 1989\end{array}$ \\
\hline Kato_03_hum & H. sapiens & di & $\begin{array}{l}\text { Traditional } \\
\text { Sanger } \\
\text { sequencing }\end{array}$ & 1,002 & $\begin{array}{l}\text { Kato et al., } \\
2003\end{array}$ \\
\hline Kato_05_hum & H. sapiens & di & $\begin{array}{l}\text { Traditional } \\
\text { Sanger } \\
\text { sequencing }\end{array}$ & 100 & Kato et al, 2005 \\
\hline yeast_mono & S. cereviseae & mono & $\begin{array}{l}\text { Traditional } \\
\text { Sanger } \\
\text { sequencing }\end{array}$ & 199 & $\begin{array}{l}\text { Segal et al., } \\
2006\end{array}$ \\
\hline
\end{tabular}

\section{2.viii Analysis of tiling array}

Preliminary analyses of tiling data were carried out using the Affymetrix Tiling Array Software (TAS) ${ }^{\circledR}$. 
Chapter 3

The characteristics of Arabidopsis nucleosome sequences 


\subsection{Introduction}

\section{$\underline{3.1 .1 \text { Introduction to nucleosome positioning signals }}$}

Early evidence for the non-random positioning of nucleosomes arose from the visualisation of chromatin isolated from chicken erythrocyte, chicken liver and cultured calf cells by electron microscopy (Oudet et al., 1975). It was demonstrated that chromatin consists of a flexible chain of repeating spherical structures (nucleosomes), separated by thin filaments of varying length (DNA linkers). Since then, there has been great interest in discovering what are the determinants of nucleosome positioning.

One of the first mononucleosome sequence datasets produced was from chicken erythrocytes (Drew and Travers, 1986). DNaseI digestion of reconstituted nucleosomes revealed non-random positioning of dinucleotides around the histone octomer, with $[\mathrm{AA} / \mathrm{TT}]$ dinucleotides tending to be positioned with minor grooves facing in toward the histone octomer. The $[\mathrm{CC} / \mathrm{GG}]$ dinucleotides were found to be positioned with the minor grooves facing out. The dinucleotides were positioned with a periodicity of $10.17 \mathrm{bp}$, and thought to be related to the helical twist.

The chicken erythrocyte sequences were further analysed to determine sequence periodicities (Satchwell et al., 1986). Fourier analysis of the position of the dinucleotides along the nucleosome sequences confirmed a periodicity of $10.2 \mathrm{bp}$ for the dinucleotides $[\mathrm{AA} / \mathrm{TT}]$ along the nucleosome core sequence at positions where the minor groove faces inward towards the histone octomer. In addition, periodicities were also seen for [CC/GG] and [TG/CA] dinucleotides, both with an opposite phasing to the [AA/TT]. However, the number of sequences was too small to reliably quantify periodicity for the other dinucleotides.

The periodic dinucleotide sequence patterns of preferred nucleosome formation sites do not occur along the length of the nucleosomal DNA at positions 10 bp apart, but the characteristic distribution observed is the result of the cumulative addition of many distributions. This can make nucleosome positioning signals difficult to detect in small samples. To overcome this, one approach was adopted whereby sequences 
were aligned by the position of the $[\mathrm{AA} / \mathrm{TT}]$ dinucleotides along nucleosome sequences collected from the literature (Ioshikhes et al., 1996). A total of five different algorithms were used to detect the $[\mathrm{AA} / \mathrm{TT}]$ repeat, which was demonstrated to have a periodicity of $10.3( \pm 0.2) \mathrm{bp}$. The alignments also demonstrated a symmetrical pattern along the length of the DNA molecule. The occurrence of a dinucleotide resulted in the opposite dinucleotide in the complementary position along the same strand of DNA, for example, [AA] repeats at positions 18 and 131-132 had [TT] counterparts at positions 127-8 and 14. The alignments also demonstrated counter-phase behaviour of the [AA/TT] dinucleotides at roughly half-phase (or $\sim 5.5 \mathrm{bp}$ ) from each other ([AANNNTTNNN $]_{\mathrm{n}}$ ).

It is thought that the counter-phase dinucleotide pattern discriminates flexible DNA from curved DNA, since the wedge angles for alternate [AA] and [TT] dinucleotides change the direction of the DNA molecule every 5-6 bases (Ioshikhes et al., 1996). Furthermore, the in-phase dinucleotide periodical repeat is thought to correspond to the curvature of DNA (Bolshoy et al., 1991, Cohanim et al., 2006).

The $[\mathrm{AA} / \mathrm{TT}]$ nucleosome positioning signal has since been found in nucleosome sequences from many species including S. cerevisiae (Albert et al., 2007; Peckham et al., 2007; Segal et al., 2006), C. elegans (Johnson et al., 2006) and D. melanogaster (Mavrich et al., 2008a). However, it should be noted that while [AA/TT] dinucleotides were found at nucleosome positions in the D. melanogaster genome, the $[\mathrm{CC} / \mathrm{GG}]$ dinucleotide distribution patterns appeared to be a more efficient predictor of nucleosome occupancy.

Whilst the $[\mathrm{AA} / \mathrm{TT}]$ periodically repeating pattern has been most extensively studied, other dinucleotide distribution patterns contribute to nucleosome positioning. Kato et al. (2003) isolated 1,002 dinucleosome sequences from human K562 cells. Following alignment and analyses of these sequences, a dinucleotide repeating pattern consisting of $[\mathrm{RR} / \mathrm{YY}]$ dinucleotides with a periodicity of $10.38 \mathrm{bp}$ was observed. The $[\mathrm{AA} / \mathrm{TT}]$ dinucleotide signal, whilst present, was not the largest contributor to the pattern. Further analysis of this set of human dinucleosome sequences revealed a stronger preference for $[\mathrm{CC} / \mathrm{GG}]$ dinucleotide repeats than [AA/TT] (Kogan et al., 2006). A recent study of nucleosome DNA fragments from 
the sheep $\beta$-lactoglobulin gene also showed a preference for [CC/GG] periodic repeats (Fraser et al., 2009).

The differences between species for nucleotide position motifs suggest that there may be a species bias toward which particular dinucleotide has a stronger effect on nucleosome positioning. This is important if the third base in a codon does have a role in ensuring the maintenance of the dinucleotide pattern, as the third base of codons are species determined (Cohanim et al., 2006). Further evidence for a species bias in nucleosome positioning signals arose from alignments of sequences around splice-site junctions (Kogan and Trifonov, 2005). These sequences, when alignments were restricted by species, revealed a preference for [CC/GG] dinucleotide patterns in human and mouse sequences and $[\mathrm{AA} / \mathrm{TT}]$ preferences in Arabidopsis and C. elegans.

Successive competitive reconstitution experiments used mouse nucleosomal DNA, isolated nucleosome sequences from the most strongly-positioned nucleosome in mouse chromatin (Widlund et al., 1997). The nucleosome DNA sequences revealed five classes of nucleosome positioning preferences including repeating [CA] dinucleotides, repeating [CAG] trinucleotides, and [TATA] tetrads [TATAA(A/C)CG(T/C)C] which represent the strongest positioning motif isolated in vivo. Fluorescent in-situ hybridisation of the nucleosomal sequences from mouse metaphase chromosomes revealed these sequences occur around the centromeric region (Widlund et al., 1997). 


\subsubsection{Aims of study in this chapter}

To date, the studies of nucleosome positioning signals have involved mammalian, insect and yeast genomes. Plant genomes have yet to be studied. With this in mind, the following hypotheses were formulated:

1. Arabidopsis nucleosome sequence composition is different from the genome average and there are differences in nucleosome and linker DNA.

2. Arabidopsis nucleosome sequences display specific distributions in their dinucleotide complement which correspond to those known to contribute to the bending of DNA around the nucleosome.

3. Arabidopsis nucleosome sequence preferences are similar to those discovered in other taxa.

To test the hypotheses above, the approach taken here involved sequencing, employing different technologies, of mono and dinucleosome fragments isolated from Arabidopsis chromatin. The different technologies applied allowed increasing resolution of the nucleosome positions. 


\subsection{Methods}

Sequence processing and PERL scripts are described in Chapter 2, Table 2.1.

\subsubsection{Determination of end-dinucleotides at the site of MNase activity}

The frequency of the occurrence of each dinucleotide at the active site of MNase was determined using a PERL script: [end_dinuc.pl]. Dinucleotides from both ends of each sequence were counted, the nucleotide at the edge being considered the first base regardless of which end of the sequence it had originated from.

\subsubsection{Calculation of observed/expected ratios for dinucleotides at sites of MNase activity}

The dinucleotide occurrence for each dinucleotide in the genome was counted using a PERL script: [count_dinuc.pl].

The expected dinucleotide values for each dinucleotide were calculated by :

$\frac{\sum X_{1} X_{2}}{n} \quad$ Equation 3.1

where $\mathrm{X}_{1}=$ base $1, \mathrm{X}_{2}=$ base 2 , so that $\sum X_{1} X_{2}$ is the sum of occurrence at base 1 multiplied by occurrence at base 2, moving along the forward strand of genome, for consecutive pairs of bases $X_{1}$ and $X_{2}$; and $n$ is the total number of dinucleotides in the genome.

The ratio of the observed occurrence for a sequence to that expected for the whole genome was calculated as:

$R=\left(\frac{\sum X_{1} X_{2}(\text { seq })}{n_{(\text {seq })}}\right) /\left(\frac{\sum X_{1} X_{2}(\text { genome })}{n_{(\text {genome })}}\right)$

Equation 3.2

where the numerator is the observed proportion of occurrence in a sequence.

The statistical significance was determined using a Chi-squared test. 
Characteristics of nucleosome sequences

The Arabidopsis Col-0 reference genome was downloaded from the TAIR (version 8) website (url at: www.arabidopsis.org). Random Arabidopsis sequences of length 147 bp were generated using a PERL script: [rand_seq.pl].

The human genome (v36.3), chicken genome (v2.1), D. melanogaster genome and $S$. cerevisiae genome were downloaded from the National Centre for Biotechnology Information (NCBI) ftp site (url at: ftp://ftp.ncbi.nih.gov/genomes/).

\section{$\underline{\text { 3.2.3 Calculation of GC content }}$}

GC content of DNA fragments within each dataset was calculating using a PERL script: [count_gc.pl]. Mann-Whitney U tests of the medians were performed using GenStat ${ }^{\circledR}\left(2008,11^{\text {th }}\right.$ Edition, VSN International, UK).

Arabidopsis BLAST datasets (TAIR version 8) were downloaded from the TAIR website (url at: www.arabidopsis.org).

\subsubsection{Determination of dinucleotide frequencies}

Dinucleotide frequencies of nucleosome sequences were determined as described in section 3.2.2. Observed values were calculated from the frequencies of each individual base within that sequence. Observed/expected ratios for each sequence were calculated as:

$$
R=\left(\frac{\sum X_{1} X_{2(s e q)}}{n_{(s e q)}}\right) /\left(\left(\frac{\sum X_{1}(s e q)}{n_{(s e q)}}\right) \times\left(\frac{\sum X_{2}(s e q)}{n_{(s e q)}}\right)\right)
$$

Equation 3.3

where $X_{1}=$ base $1, X_{2}=$ base 2 moving along the sequence of the forward strand for pairs of bases $X_{1}$ and $X_{2}$; and $n=$ DNA fragment length. 


\subsubsection{Distribution of dinucleotides along the nucleosome DNA fragment}

The positions of each dinucleotide within nucleosome sequences were determined using a PERL script: [pos_dinuc.pl]. Frequency tables were constructed using a PERL script: [count_freqs.pl].

\subsubsection{Periodicity of dinucleotide distribution.}

Fourier analysis and autocorrelation tests of the frequency tables (calculated in 3.2.5) used the 'periodtest' and time series procedures in Genstat v11. Fourier analysis is described in Chapter two, Section 2.2.iv.

\subsubsection{Construction of a nucleosome sequence database}

Nucleosome sequence data were collected from various publicly-available sources and used to populate a relational nucleosome positioning database implemented in Microsoft Access. Data sources are summarised in Table 3.1 and an entityrelationship diagram of the database is shown in Figure 3.1.

Table 3.1 Sources of publicly-available nucleosome sequence data collected and used to populate a nucleosome sequence database.

\begin{tabular}{|c|c|c|c|c|c|}
\hline$\overline{\text { Data source }}$ & Format & $\begin{array}{l}\text { No. of } \\
\text { sequences }\end{array}$ & Method of isolation & $\begin{array}{l}\text { Year } \\
\text { entered } \\
\text { into } \\
\text { database }\end{array}$ & Reference \\
\hline $\begin{array}{l}\text { Andrew Travers } \\
\text { (personal } \\
\text { communication) }\end{array}$ & $\begin{array}{l}\text { MS } \\
\text { Excel }\end{array}$ & 177 & $\begin{array}{l}\text { MNase digestion of } \\
\text { chromatin }\end{array}$ & 2006 & $\begin{array}{l}\text { Satchwell and } \\
\text { Travers (1989) }\end{array}$ \\
\hline $\begin{array}{l}\text { Andrew Travers } \\
\text { (personal } \\
\text { communication) }\end{array}$ & $\begin{array}{l}\text { MS } \\
\text { Excel }\end{array}$ & 157 & $\begin{array}{l}\text { MNase/S1 } \\
\text { digestion of } \\
\text { chromatin }\end{array}$ & 2006 & $\begin{array}{l}\text { Drew and } \\
\text { Travers (1986) }\end{array}$ \\
\hline $\begin{array}{l}\text { I. Ioshikhes } \\
\text { (via Graham } \\
\text { King) }\end{array}$ & FASTA & 207 & $\begin{array}{l}\text { Mixture of } \\
\text { MNase/DNase1 } \\
\text { isolated nucleosomes }\end{array}$ & 2006 & $\begin{array}{l}\text { Ioshikhes et al. } \\
\text { (1996) }\end{array}$ \\
\hline $\begin{array}{l}\text { NPRD } \\
\text { (Download) }\end{array}$ & FASTA & 100 & $\begin{array}{l}\text { Mixture of } \\
\text { MNase/DNase1 } \\
\text { isolated nucleosomes }\end{array}$ & 2006 & $\begin{array}{l}\text { Levitsky et al. } \\
\text { (2005) }\end{array}$ \\
\hline
\end{tabular}




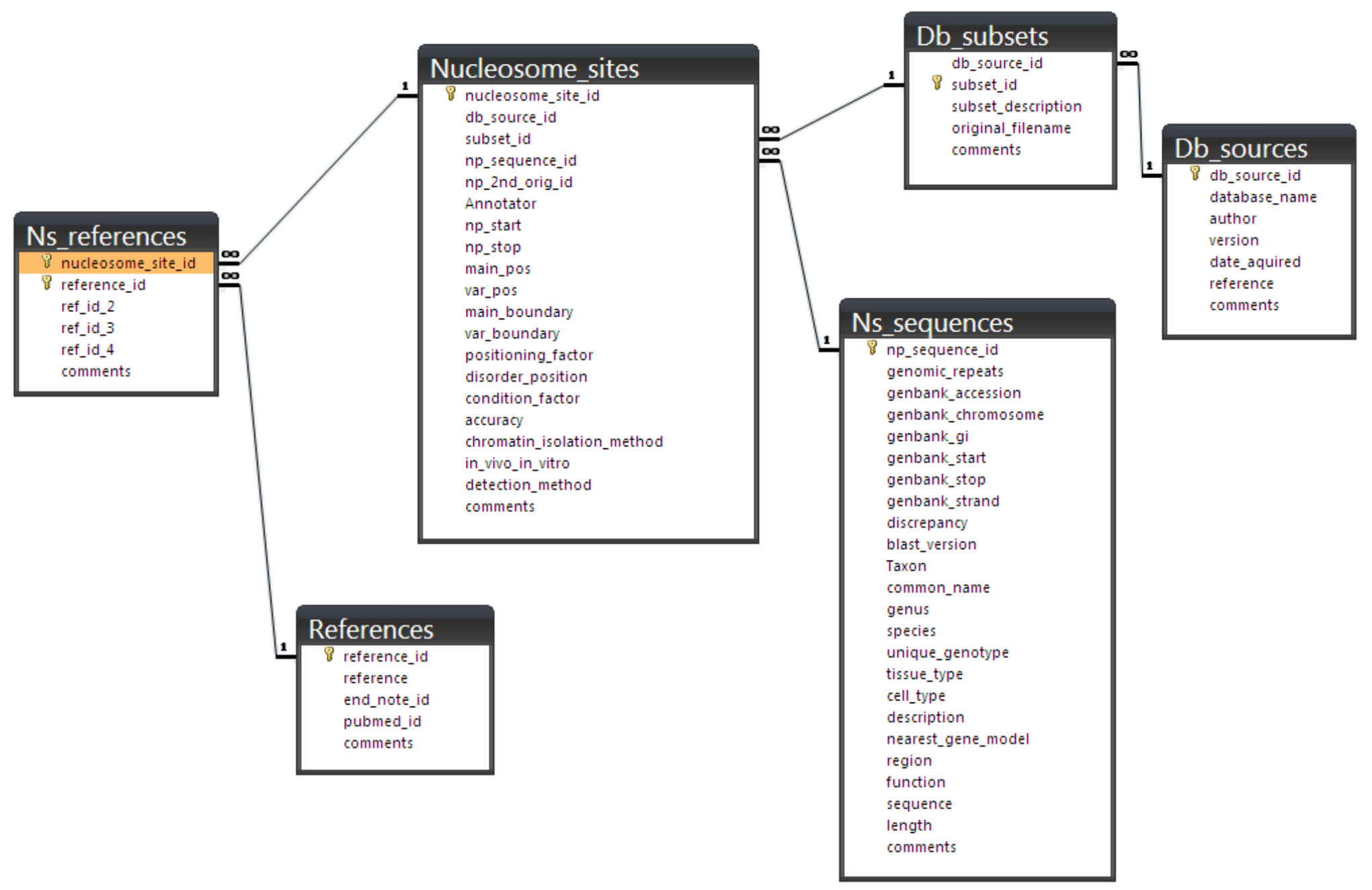

Figure 3.1 Entity-relationship diagram showing tables and fields of the relational nucleosome sequence database. 


\subsection{Results}

Much effort was expended in attempting to acquire nucleosome positioning data from the Arabidopsis tiling arrays, with four (nucleosome) datasets and one control dataset being generated. Following preliminary analysis of the tiling array datasets, a nucleosome signal which was uniformly distinct from the control could not be detected, and therefore the results are not discussed.

\subsection{1.i Generation of Arabidopsis nucleosome DNA fragments}

Sequencing of nucleosome DNA fragments isolated from Arabidopsis leaf tissue chromatin resulted in four datasets. An overview of this is shown in Table 3.2.

Table 3.2 Arabidopsis datasets generated in this study

\begin{tabular}{|c|c|c|c|c|c|c|}
\hline Dataset & Origin & $\begin{array}{l}\text { Raw } \\
\text { sequencing } \\
\text { reads }\end{array}$ & $\begin{array}{l}\text { Reads } \\
\text { aligned }\end{array}$ & Unique hits & $\begin{array}{c}\text { Mono- } \\
\text { nucleosome }\end{array}$ & $\begin{array}{c}\text { Di- } \\
\text { nucleosome }\end{array}$ \\
\hline Ath_01_wt & WT_clone & 576 & 550 & 476 & N/A & 476 \\
\hline Ath_02_wt & WT 454 & 43,420 & 25,227 & 19,548 & 19,548 & N/A \\
\hline \multirow[t]{2}{*}{ Ath_03_wt } & WT (F) & $1,536,234$ & $1,097,364$ & 908,572 & $16,589 *$ & $924,381 *$ \\
\hline & WT (R) & $1,536,234$ & & & & \\
\hline \multirow[t]{2}{*}{ Ath_04_MET1 } & $M E T 1(\mathrm{~F})$ & $2,543,279$ & $1,702,915$ & $1,280,099$ & $1,054,734 *$ & $98,365^{*}$ \\
\hline & $M E T 1(\mathrm{R})$ & $2,543,279$ & & & & \\
\hline \multirow[t]{2}{*}{ Ath_05_wt } & WT (F) & & & & $3,557,287$ & \\
\hline & & & & & & \\
\hline
\end{tabular}

*including repeats, F: forward DNA strand, R: reverse DNA strand

\subsection{1.ii Dinucleosome DNA fragments from a clone library}

A dinucleosome library, containing over 3,000 clones, was constructed from Arabidopsis leaf tissue. A total of 576 cloned fragments were sequenced. Dinucleosome DNA fragments were aligned to the Arabidopsis Col-0 reference genome (TAIR 8) using the Time-Logic Tera-probe BLAST (Smith-Waterman algorithm) alignment on a DeCypher server. Matches with $97 \%$ or greater similarity to the reference genome and over $294 \mathrm{bp}$ in length were included in the final set of 476 fragments. This set was nominated as Ath_01_wt. 
Fragments which were less than 294 nucleotides in length were removed from the final set to give the set for analysis. The theoretical minimum nucleotide length of a dinucleosome fragment is assumed to be 294 nucleotides, which is the length of two nucleosomes $(1$ nucleosome $=147$ nucleotides) $($ Richmond and Davey, 1993). However, this does not take into consideration minimum linker length or steric hindrance. In addition, fragments which aligned to organelle genomes were also removed from all datasets, since organelles do not form true nucleosomes in vivo (Salganik et al., 1991).

\subsection{1.iii Dinucleosome DNA fragments sequenced by 454 FLX technology}

Arabidopsis dinucleosome DNA was sequenced using a 1/8th 454 pico-titre plate. This generated a collection of 43,420 sequence reads, varying from 41 to 368 nucleotides in length. The DNA sequences were aligned to the Arabidopsis Col-0 reference genome (TAIR8), as in the previous section (3.3.1.ii). Fragments which aligned with $99 \%$ similarity or more were included in the final set. Fragments which aligned to more than one genomic region (genomic repeats) were omitted, leaving a final set of 19,548 fragments. This set was nominated as Ath_02_wt.

Since 454 FLX sequence reads do not extend to dinucleosome length, fragments were trimmed to 147 nucleotides from the 5' end and treated as mononucleosome fragments in further analyses, as shown in the schematic Figure 3.2. Length distributions of datasets Ath_O1_wt and Ath_O2_wt are shown in Figure 3.3.

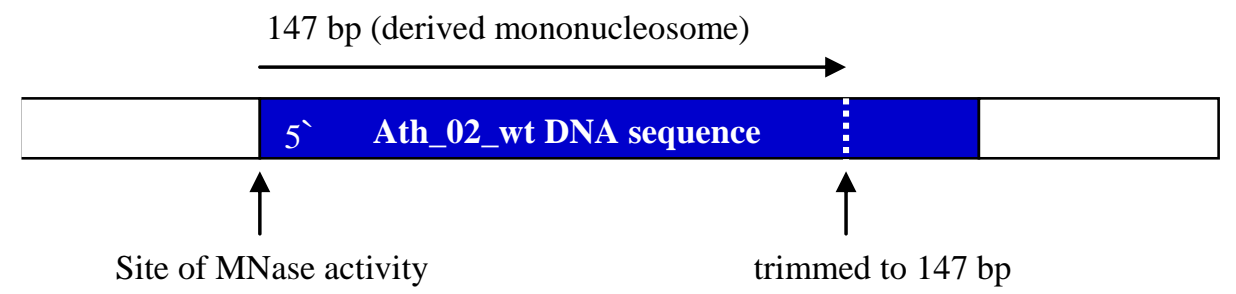

Figure 3.2 Schematic showing the processing of Arabidopsis dinucleosome fragments from dataset $\boldsymbol{A t h \_ 0 2 \_ w t}$. Sequences were trimmed to $147 \mathrm{bp}$ from the sites of MNase activity and treated as mononucleosomes. 

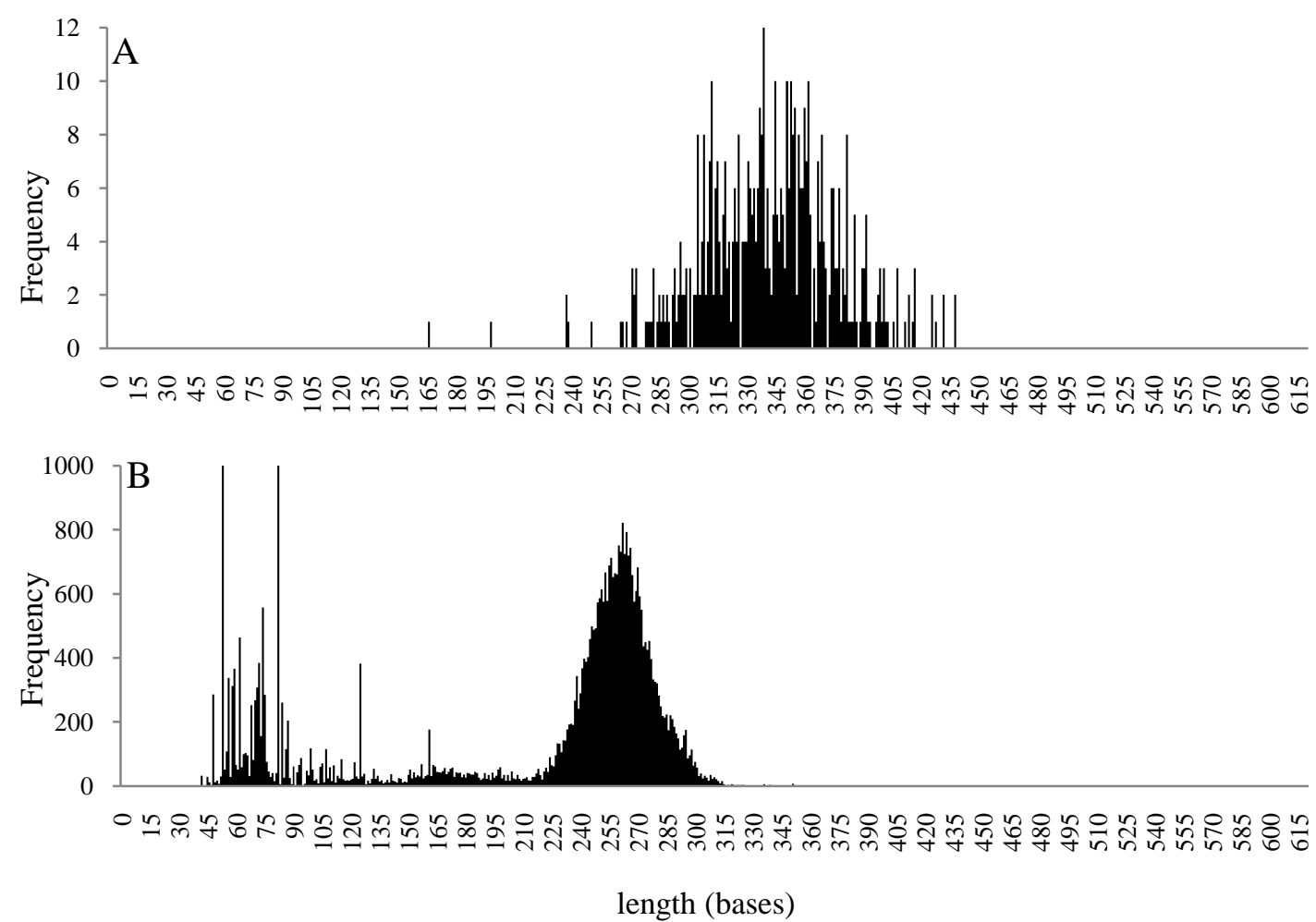

Figure 3.3 Distributions of dinucleosome DNA fragment lengths, A: length distribution of the nucleosome fragments of dataset Ath_01_wt. B: shows the length distribution of sequences from dataset Ath_O2_wt, prior to trimming to $147 \mathrm{bp}$.

\subsection{1.iv Nucleosome DNA fragments sequenced using Illumina (Solexa) technology}

A total of $2 \mu \mathrm{g}$ each of Arabidopsis wild-type (Col-0) and anti-sense MET1 (C24 ecotype) mono (MET1) and dinucleosome (wild-type) fragments were sequenced using the Illumina Genome Analyzer sequencing technology, on 1/8 th of a channel. For an experiment where samples are mixed and sequenced in the same channel, fragments are tagged with a $4 \mathrm{bp}$ 'barcode' which allows the samples to be identified and sorted following the sequencing reactions. The barcode and primer account for $18 \mathrm{bp}$ of the sequence. The nucleosome fragment sequences obtained were thus 32 bp from each end of the fragment (see schematic Figure 3.4.). A total of 1,536,234 pairs of reads (paired having read from each end of the fragment) were generated for wild-type and 2,5432,79 pairs of reads were generated for anti-sense MET1.

Both mononucleosome and dinucleosome DNA fragments were isolated from the wild-type and anti-sense MET1 tissues and pooled. For sequencing, the Solexa project was outsourced to and external company. Unfortunately, only the mononucleosome 


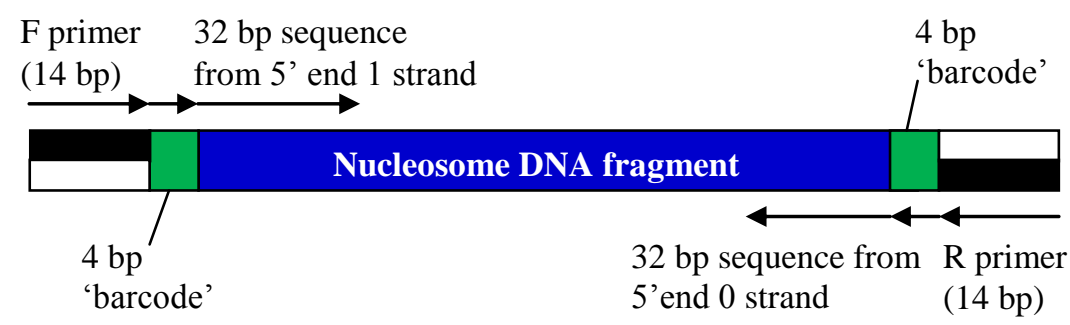

Figure 3.4 Schematic showing the process of paired-end Illumina sequencing of Arabidopsis nucleosome DNA fragments. Different $4 \mathrm{bp}$ barcodes are applied to each sample when multiple samples are mixed prior to sequencing to allow for sorting later.

fragments from the anti-sense MET1 dataset and dinucleosome fragments from the wild-type following excision of fragments from agarose gels. It was decided to complete the analysis of nucleosome positions with these fragments. However it became clear later that a further dataset was required to enable comparisons between the wild-type and anti-sense MET1 datasets. Therefore a further wild-type mononucleosome dataset was generated, but due to the late arrival of this dataset, it will be presented here, but not discussed until Chapter 5 .

The Solexa DNA sequence fragments were aligned to the Arabidopsis Col-0 reference genome, (TAIR8), as in 3.3.1.ii. Positions were assigned to pairs of sequences where they were aligned with $100 \%$ sequence similarity to the Arabidopsis reference genome, the pairs being aligned within 620 nucleotides from each other and on opposite DNA strands. This resulted in a total of 1,097,364 wildtype and 1,702,915 anti-sense MET1 (di) nucleosome positions. These sets were nominated Ath_03_wt and Ath_04_MET1, respectively. A further wild-type mononucleosome dataset was nominated Ath_05_wt (n = 3,557,287). The length distributions of Ath_03_wt, Ath_04_MET1 and Ath_05_wt are shown in Figure 3.5.

Datasets Ath_03_wt and Ath_04_MET1 contain all aligned nucleosome sequences. Subsets of each dataset were constructed, where sequences that aligned to a single genomic region were defined as unique hits as shown in Table 3.2. For sequence analysis, all sequences which aligned to nuclear chromosomes 1-5 were used for each dataset. For the purposes of analysis, nucleosome and linker regions were separated by splitting the dinucleosome sequences $147 \mathrm{bp}$ from the $5^{\prime}$ and 3 ' ends as 
Characteristics of nucleosome sequences

shown in the schematic (Figure 3.6). The resulting fragments were nominated as (WT/MET1)_left,(WT/MET1)_di_linker and (WT/MET1)_right.
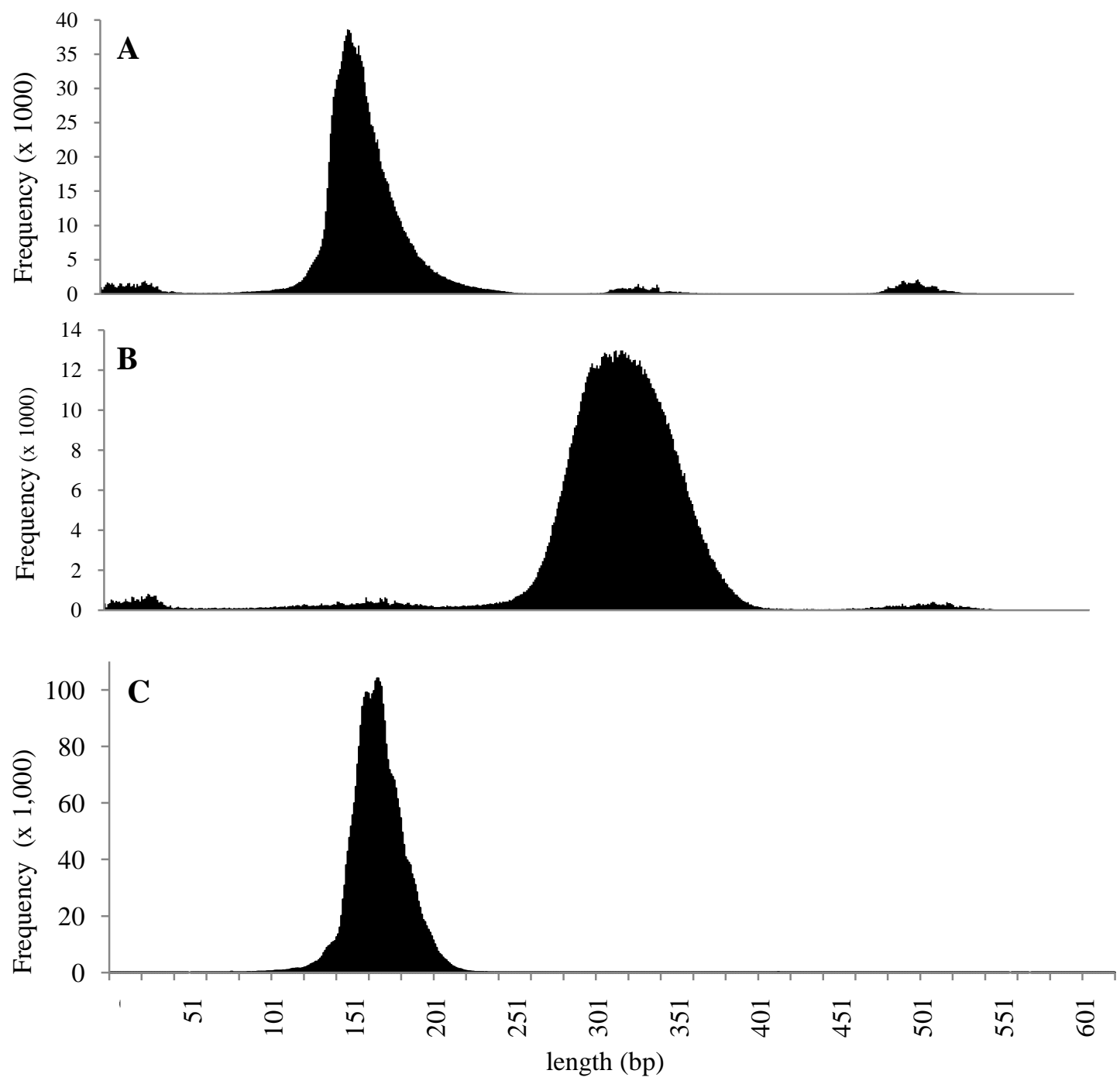

Figure 3.5 Length distribution of mono and di nucleosome sequences of datasets A: Ath_04_MET1, B:Ath_03_wt and C:Ath_05_wt

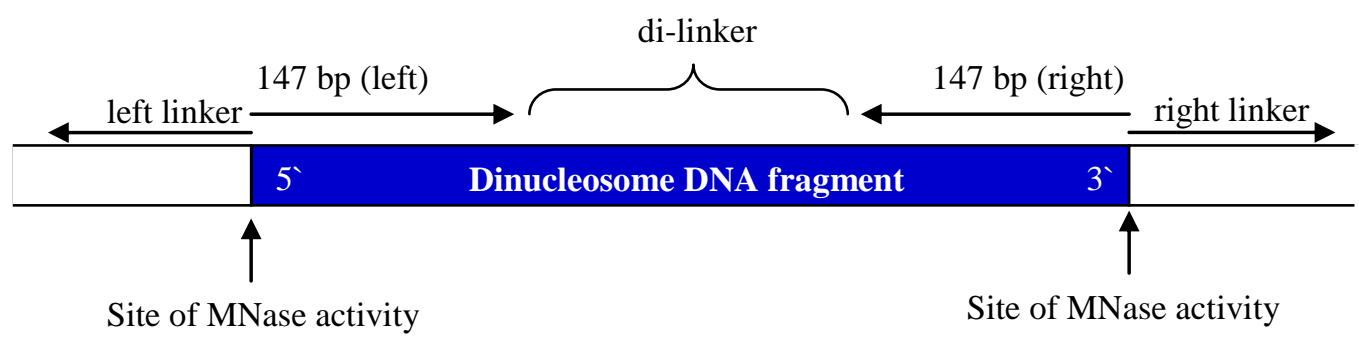

Figure 3.6 Schematic showing the division of dinucleosome sequences from datasets Ath_03_wt and Ath_04_MET1 into nucleosome and linker, and the generation of derived linker datasets. 
Characteristics of nucleosome sequences

Mononucleosome fragments were identified as fragments between 130 - $177 \mathrm{bp}$ in length, and named the 'WT_mono' and 'MET1_mono' datasets for wild-type and anti-sense $M E T 1$, respectively. In addition, the sequences $52 \mathrm{bp}$ to the left and right of the nucleosome DNA fragments were collected, and allocated into 'left_linker' and 'right_linker' datasets, respectively.

For the purposes of sequence analysis, the wt_left (Table 3.3) and MET1_mono datasets were used, in order for the wild-type and anti-sense MET1 datasets to be of a comparable size. For comparison to genomic DNA sequences, a random set of 584,032 sequences between 147 and $150 \mathrm{bp}$ in length were generated from the Arabidopsis Col-0 reference genome (TAIR8) using PERL scripts: rand_seq.pl and find_seq.pl (section 3.2.2). A summary of the derived datasets is shown in Table 3.3.

Table 3.3 A summary of datasets derived from Ath_03_wt and Ath_04_MET1

\begin{tabular}{|c|c|c|c|}
\hline Ath derived & Description & Length (bp) & $\begin{array}{l}\text { No of } \\
\text { sequences }\end{array}$ \\
\hline$w t$ left & $\begin{array}{l}147 \text { bp from } 5 \text { ' edge dinucleosome } \\
\text { fragment (+ strand) }\end{array}$ & 147 & 924,381 \\
\hline$w t$ right & $\begin{array}{l}-147 \text { bp from 3' edge dinucleosome } \\
\text { fragment (- strand) }\end{array}$ & 147 & 924,381 \\
\hline$w t \_m o n o$ & DNA fragments $>130 \mathrm{bp}<177 \mathrm{bp}$ & $130-177$ & 16,589 \\
\hline$w t$ di-linker & $\begin{array}{l}\text { DNA fragment remaining from } \\
\text { dinucleosome after } \\
\text { subtraction of left and right nucleosome }\end{array}$ & $10-326$ & 924,381 \\
\hline MET1_left & $\begin{array}{l}147 \text { bp from 5' edge dinucleosome } \\
\text { fragment (+ strand) }\end{array}$ & 147 & 98,365 \\
\hline MET1_right & $\begin{array}{l}-147 \text { bp from 3' edge dinucleosome } \\
\text { fragment (- strand) }\end{array}$ & 147 & 98,365 \\
\hline MET1_mono & DNA fragments $>130 \mathrm{bp}<177 \mathrm{bp}$ & $130-177$ & $1,054,734$ \\
\hline MET1_di-linker & $\begin{array}{l}\text { DNA fragment remaining from } \\
\text { dinucleosome after } \\
\text { subtraction of left and right nucleosome }\end{array}$ & $10-326$ & 98,365 \\
\hline wt_left_linker & -52 bp from 5' edge nucleosome fragment & 52 & 924,381 \\
\hline wt_right_linker & +52 bp from 3' edge nucleosome fragment & 52 & 924,381 \\
\hline MET1_left_linker & -52 bp from 5' edge nucleosome fragment & 52 & 16,589 \\
\hline MET1_right_linker & +52 bp from 3' edge nucleosome fragment & 52 & 16,589 \\
\hline Ath_rand & $\begin{array}{l}\text { Randomly generated from the Arabidopsis } \\
\text { Col-0 reference genome }\end{array}$ & $147-150$ & 584,032 \\
\hline
\end{tabular}




\subsection{2.i Specificity of micrococcal nuclease}

In order to determine whether the digestion of chromatin had been affected by any bias in MNase base preference, the incidence of each base was recorded for the first and last position from the forward strand of each sequence in each Arabidopsis dataset (with the exception of Ath_03_wt, where the nucleotide at the MNase site was recorded). Nucleotides [W] accounted for between 82 and $97 \%$ of the nucleotides in the first and last positions in the Arabidopsis nucleosome fragments, with [WG] being the most favoured dinucleotide, this occurring between 40 and $49 \%$ of the Arabidopsis sequences. Interestingly, the dinucleotide [WC] occurred at a lower frequency, indicating the MNase preference in for [W] followed by [G]. However, one set (Ath_03_wt) did show an increased preference for [GT] (9\%) at the MNase cut-site when compared to the other Arabidopsis data. The percent of occurrence of [W], [WG] and [WC] are shown in Table 3.4.

Table 3.4 Frequency of [W], [WG] and [WC] at the site of MNase activity for each nucleosome dataset

\begin{tabular}{|c|c|c|c|c|}
\hline \multirow[b]{2}{*}{$\begin{array}{l}\text { Arabidopsis } \\
\text { dataset }\end{array}$} & \multirow[b]{2}{*}{$\begin{array}{l}\text { Number of ends of } \\
\text { nucleosome DNA } \\
\text { fragments }\end{array}$} & \multicolumn{3}{|c|}{ Nucleotide/dinucleotide (\% nucleosome fragments) } \\
\hline & & {$[\mathrm{W}]$} & [WG] & {$[\mathrm{WC}]$} \\
\hline Ath_01_wt & 952 & 94 & 49 & 13 \\
\hline Ath_O2_wt & 19,548 & 97 & 44 & 22 \\
\hline Ath_03_wt & $2,554,122$ & 82 & 40 & 15 \\
\hline Ath_04_MET1 & $2,306,198$ & 84 & 40 & 15 \\
\hline
\end{tabular}

To test the hypothesis that the occurrence of each dinucleotide at the site of MNase activity is not random, the observed/expected ratios were calculated (equation 3.2), comparing the occurrence of dinucleotides at the MNase cut-site to the occurrence of each dinucleotide throughout the Arabidopsis Col-0 reference (TAIR8) genome (equation 3.1). The ratios are shown in Figure 3.7. 
Characteristics of nucleosome sequences
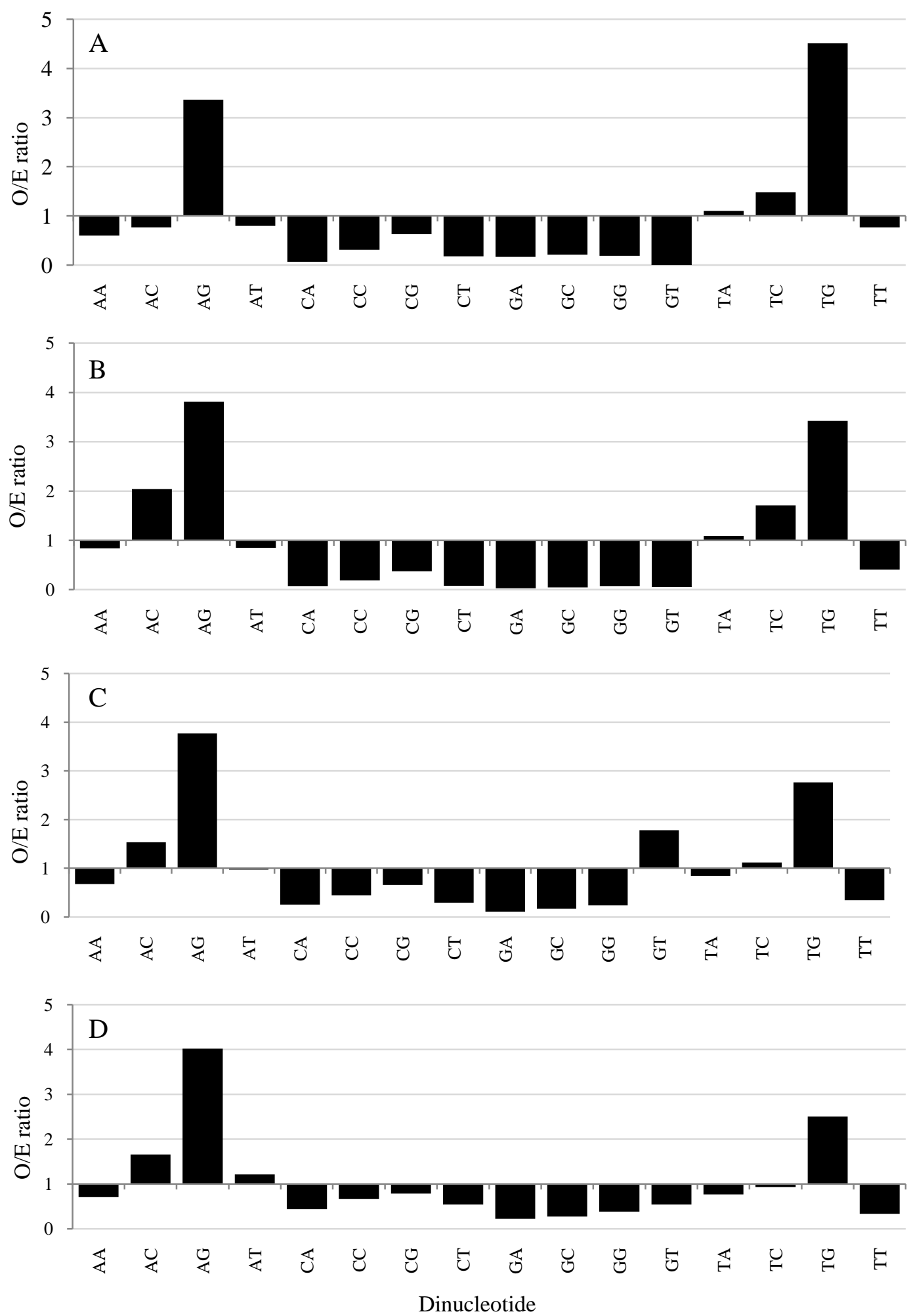

Figure 3.7 Observed/expected ratios for the occurrence of each dinucleotide at the site of MNase activity for Arabidopsis datasets, A: Ath_01_wt, B: Ath_02_wt, C: Ath_03_wt and D: Ath_04_MET1 
Characteristics of nucleosome sequences

The ratios show significantly greater than expected occurrences $(\mathrm{p}=0.05$ (ChiSquare)) of $[\mathrm{AG}]$ in all Arabidopsis datasets, and [TG] in the Ath_01_wt and Ath_02_wt datasets.

The data obtained here suggests that the Arabidopsis chromatin has a bias towards a greater than expected occurrence of [WG] at the MNase cut-site, when compared to dinucleotide occurrence throughout the whole genome. To determine if this is a unique feature of this genus, the observed/expected ratios of dinucleotide occurrence at the site of MNase activity were calculated for other taxa. Data were taken from publicly available sources (chicken (Drew and Travers, 1986), yeast (Segal et al., 2006), C. elegans (Johnson et al., 2006) and human (Kato et al., 2005, Kato et al., 2003), where the nucleosome DNA fragments were prepared by MNase digestion of chromatin. The significance of the occurrence of each dinucleotide compared to the genome was determined using a Chi-squared test. The ratios are shown in Table 3.5, significantly higher than expected occurrences are shown in bold; while significantly lower than expected occurrences are indicated with an asterisk.

There appears to be considerable variation in the observed/expected ratios between each species tested, and within the three chicken sets. The higher than expected occurrence of [WG] observed at Arabidopsis sites on MNase activity were also observed in the $C$. elegans dataset. The chicken and yeast datasets show a bias toward either $[\mathrm{AG}]$ or $[\mathrm{TG}]$, with the exception of the trimmed chicken dataset. The two human datasets show a bias towards $[\mathrm{CC}]$, $[\mathrm{CG}]$ and $[\mathrm{GG}]$ dinucleotides at the site of MNase activity, when compared to the whole human genome. As the previously reported sequence preference for MNase activity has been determined to be $[\mathrm{WG}]$, these results suggest that there may be more factors involved when chromatin is digested with this enzyme such as chromatin conformation and DNA accessibility. 
Table 3.5 Observed/expected ratios of dinucleotide occurrence at the site of MNase activity of nucleosome sequences. The Arabidopsis datasets are shown for comparison to the publicly-available data for other taxa. Chi-squared calculated using percent occurrence of dinucleotides at MNase/whole genome. Bold = significantly higher than expected; $*$ = significantly lower than expected $(\mathrm{p}=0.05)$

\begin{tabular}{|c|c|c|c|c|c|c|c|c|c|c|c|}
\hline & $\begin{array}{c}A t h \_01 \_w t \\
n=950\end{array}$ & $\begin{array}{c}\text { Ath_02_wt } \\
n=25,227\end{array}$ & $\begin{array}{c}A t h \_03 \_w t \\
n=2,194,728\end{array}$ & $\begin{array}{c}\text { Ath_O4_ } \\
\text { MET1 } \\
n=3,405,830\end{array}$ & $\begin{array}{c}\begin{array}{c}\text { chick_di_ } \\
\text { trim } \\
n=98\end{array} \\
\end{array}$ & $\begin{array}{c}\text { chick_di__ } \\
\text { untrim } \\
n=206\end{array}$ & $\begin{array}{c}\text { chick_mono } \\
\text { _untrim } \\
n=304\end{array}$ & $\begin{array}{c}\text { yeast_- } \\
\text { mono } \\
n=398\end{array}$ & $\begin{array}{c}\text { C.elegans_ } \\
\text { mono } \\
n=327,294\end{array}$ & $\begin{array}{c}\text { kato_03_- } \\
\text { human } \\
n=2004\end{array}$ & $\begin{array}{c}\text { kato_05_ } \\
\text { human } \\
n=200\end{array}$ \\
\hline AA & $0.6^{*}$ & $0.8^{*}$ & $0.7 *$ & $0.7^{*}$ & $1.5^{*}$ & $1.0^{*}$ & $1.2^{*}$ & $1.5^{*}$ & $0.5^{*}$ & 0.2 & 0.1 \\
\hline $\mathrm{AC}$ & $0.8^{*}$ & $2.0^{*}$ & $1.5^{*}$ & $1.7 *$ & 4.3 & $1.4^{*}$ & $0.7^{*}$ & $0.6^{*}$ & 2.7 & 2.8 & $2.0^{*}$ \\
\hline $\mathrm{AG}$ & 3.4 & 3.8 & 3.8 & 4.0 & $0.8^{*}$ & 4.1 & 2.1 & $2.0^{*}$ & 3.7 & 2.4 & 2.1 \\
\hline $\mathrm{AT}$ & $0.8^{*}$ & $0.9^{*}$ & $1.0^{*}$ & $1.2^{*}$ & 3.7 & 2.6 & $0.8^{*}$ & 4.0 & $0.6^{*}$ & $0.5^{*}$ & $0.3^{*}$ \\
\hline $\mathrm{CA}$ & $0.1^{*}$ & $0.1^{*}$ & $0.3^{*}$ & $0.4^{*}$ & $0.3^{*}$ & 4.5 & 3.3 & 5.0 & $0.1^{*}$ & 2.3 & 2.2 \\
\hline $\mathrm{CC}$ & $0.3^{*}$ & $0.2^{*}$ & $0.4^{*}$ & $0.7^{*}$ & $1.8^{*}$ & $0.0 *$ & $1.6^{*}$ & $0.0^{*}$ & $0.4^{*}$ & 5.2 & 5.7 \\
\hline $\mathrm{CG}$ & $0.6^{*}$ & $0.4^{*}$ & $0.7^{*}$ & $0.8^{*}$ & $2.0 *$ & $0.9 *$ & 8.1 & $0.2^{*}$ & $0.7^{*}$ & 11.0 & 15.0 \\
\hline $\mathrm{CT}$ & $0.2^{*}$ & $0.1^{*}$ & $0.3^{*}$ & $0.5^{*}$ & $1.1^{*}$ & 3.2 & 3.5 & 2.2 & $0.2^{*}$ & $1.9^{*}$ & $1.0 *$ \\
\hline GA & $0.2^{*}$ & $0.0^{*}$ & $0.1^{*}$ & $0.2^{*}$ & 3.1 & $0.9^{*}$ & 2.9 & $1.5^{*}$ & $0.1^{*}$ & 2.1 & $1.2 *$ \\
\hline GC & $0.2^{*}$ & $0.0^{*}$ & $0.2^{*}$ & $0.3^{*}$ & 2.5 & $0.4^{*}$ & $0.9^{*}$ & $0.0^{*}$ & $0.1^{*}$ & 2.3 & 2.6 \\
\hline GG & $0.2^{*}$ & $0.1^{*}$ & $0.2^{*}$ & $0.4^{*}$ & 2.6 & $0.4^{*}$ & $1.6^{*}$ & $0.0^{*}$ & $0.1^{*}$ & 4.8 & 5.7 \\
\hline GT & $0.0^{*}$ & $0.1^{*}$ & $1.8^{*}$ & $0.5^{*}$ & 6.3 & $1.3 *$ & $1.2^{*}$ & $1.2 *$ & $0.1^{*}$ & 2.9 & 2.6 \\
\hline $\mathrm{TA}$ & $1.1^{*}$ & $1.1^{*}$ & $0.8^{*}$ & $0.8^{*}$ & $0.0 *$ & $1.8^{*}$ & $1.1^{*}$ & 3.3 & $0.8^{*}$ & $0.4^{*}$ & $1.1^{*}$ \\
\hline $\mathrm{TC}$ & $1.5^{*}$ & $1.7^{*}$ & $1.1^{*}$ & $0.9 *$ & 3.4 & $0.9 *$ & $2.0^{*}$ & $1.2^{*}$ & 2.8 & 2.6 & 3.7 \\
\hline $\mathrm{TG}$ & 4.5 & 3.4 & 2.8 & 2.5 & $0.3^{*}$ & 4.1 & 3.8 & 5.0 & 3.5 & $1.9^{*}$ & $1.9^{*}$ \\
\hline $\mathrm{TT}$ & $0.8^{*}$ & $0.4^{*}$ & $0.3^{*}$ & $0.3^{*}$ & $1.3 *$ & $1.2 *$ & $1.5^{*}$ & $0.8^{*}$ & $0.4^{*}$ & 0.2 & $0.2 *$ \\
\hline
\end{tabular}




\subsection{2.ii The GC content of nucleosomal DNA fragments}

To test the hypothesis that nucleosomal DNA has a higher GC content than the average for the genome, the GC content of each sequence was calculated for the $w t$ left, MET1_mono and random genomic sequences subsets. The distribution of GC content (expressed as a percentage) for both subsets is shown in Figure 3.8.

The mean GC contents of the wt-left, MET1_mono and Ath_rand subsets were $41.3 \%, 40.1 \%$ and $36.0 \%$, respectively. The GC contents of both nucleosome DNA subsets were higher than the mean for the random genomic sequences and the genome average of $36 \%$.

As genic regions of the Arabidopsis genome are known to have higher GC content than the intergenic regions, GC contents were calculated for Arabidopsis exons, introns, 5'-UTR's, 3'-UTR's, transposable elements and intergenic sequences. The nucleosome sequences have a GC content most similar to the exon subset $(41.3 \%$ for the wt_left compared to $42.5 \%$ for the exon), and all other subsets have lower GC contents. This suggests that the nucleosome datasets are likely to be enriched for exon sequences.

In order to test whether there were statistically significant differences between the GC contents of the nucleosome DNA datasets and the Arabidopsis genomic subsets, two-sample Mann-Whitney-U tests were performed. Each Arabidopsis genomic subset was tested against the GC content of wt_left and MET1_mono.

All tests showed significant differences between medians, although the extreme size of the datasets tested may have influenced the outcome of the test. The results of the Mann-Whitney-U tests are shown in Table 3.6. 
Characteristics of nucleosome sequences
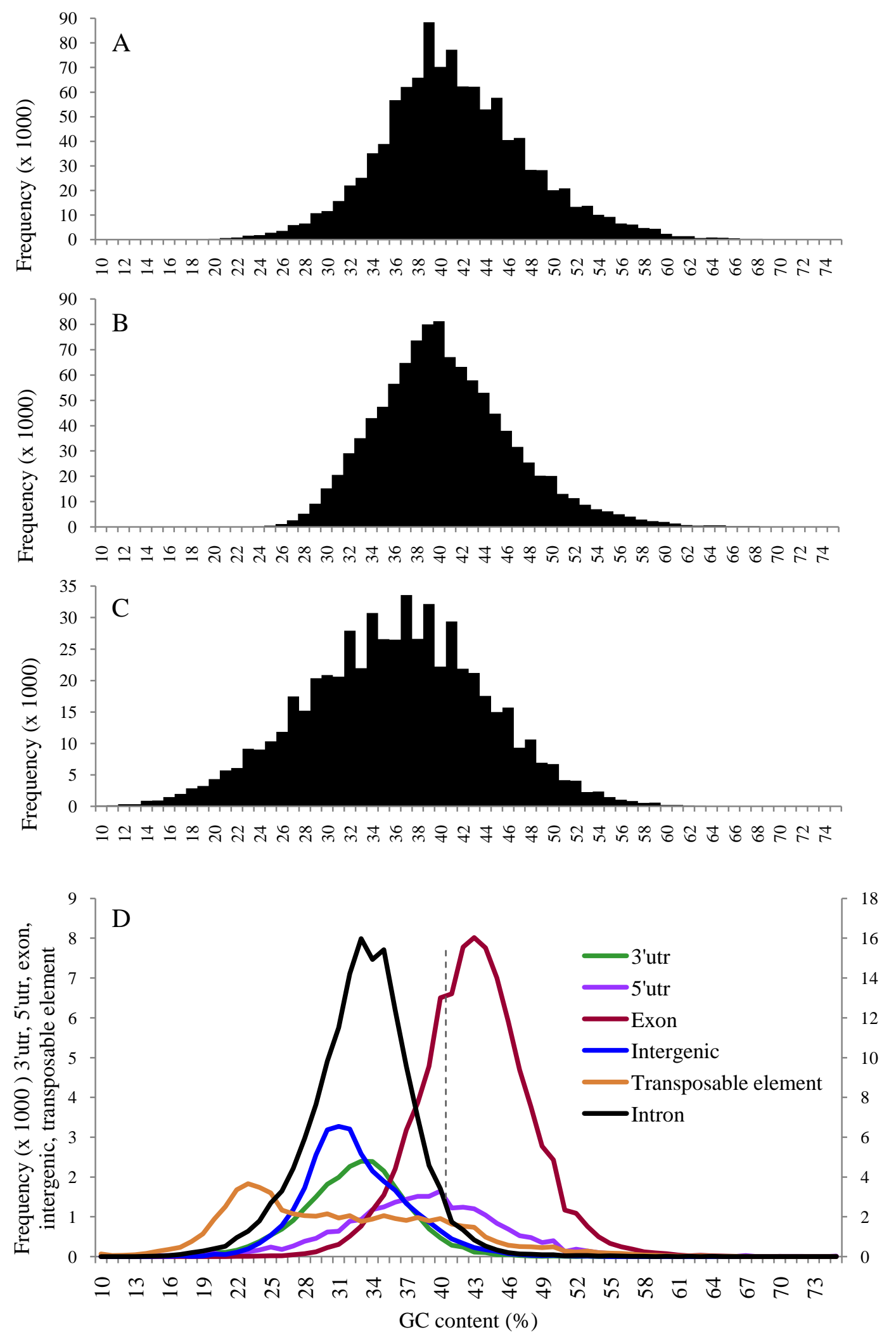

Figure 3.8 Distribution of GC content (\%) for each sequence in the Arabidopsis datasets A: wt_left nucleosome DNA, B: MET1_mono nucleosome DNA, C: Ath_rand and D: Arabidopsis TAIR 8 blast sets (3'UTR's, 5'UTR's, exons, introns, transposable elements and intergenic sequences). The dashed line indicates the mean for wt_left nucleosomes. The distribution of intron sequences is on the right-hand $y$-axis for clarity. 
Table 3.6 Mean, standard deviation and median of the distribution of GC content (\%) of wt_left and MET1_mono nucleosome sequences (highlighted in yellow) and the Arabidopsis (TAIR 8) genomic subsets (exon, intron, 3'UTR, 5'UTR, transposable elements and intergenic DNA sequences). In addition, the results of Mann-Whitney tests (p-values) of each genomic subset vs. wt_left and MET1_mono are reported, testing the null hypothesis that the medians are equal.

\begin{tabular}{|c|c|c|c|c|c|c|c|}
\hline Fragment subset & $\begin{array}{l}\text { mean } \\
(\bar{y})\end{array}$ & stdev & median & $\begin{array}{l}w t_{\text {_left }} \\
\text { test } \\
\text { statistic }\end{array}$ & $\begin{array}{l}w t \text { left } \\
\text { p-value }\end{array}$ & $\begin{array}{l}\text { MET1_mo } \\
\mathrm{n} \\
\text { test } \\
\text { statistic }\end{array}$ & $\begin{array}{l}M E T 1 \_m \\
\text { on } \\
\text { p-value }\end{array}$ \\
\hline Exon $(n=154,240)$ & 42.5 & 4.9 & 42.5 & 73.71 & $\mathrm{p}<0.001$ & 137.41 & $\mathrm{p}<0.001$ \\
\hline$w t \_l e f t$ & 41.3 & 6.6 & 41.0 & & & & \\
\hline MET1_mono & 40.1 & 6.2 & 39.6 & & & & \\
\hline 5'UTR $(n=24,267)$ & 38.0 & 7.9 & 38.0 & 71.10 & $\mathrm{p}<0.001$ & 44.45 & $\mathrm{p}<0.001$ \\
\hline Ath_rand & 36.0 & 8.0 & 36.3 & 405.81 & $\mathrm{p}<0.001$ & 316.67 & $\mathrm{p}<0.001$ \\
\hline Intron $(n=86,850)$ & 32.6 & 4.6 & 32.8 & 477.06 & $\mathrm{p}<0.001$ & 441.89 & $\mathrm{p}<0.001$ \\
\hline $3^{\prime} \mathrm{UTR}(n=25,273)$ & 32.1 & 4.9 & 32.2 & 209.44 & $\mathrm{p}<0.001$ & 196.47 & $\mathrm{p}<0.001$ \\
\hline $\begin{array}{l}\text { Intergenic } \\
(n=31,089)\end{array}$ & 32.0 & 4.6 & 31.5 & 231.41 & $\mathrm{p}<0.001$ & 218.93 & $\mathrm{p}<0.001$ \\
\hline $\begin{array}{l}\text { Transposable } \\
\text { element }(n=31,060)\end{array}$ & 30.7 & 9.3 & 29.4 & & & & \\
\hline
\end{tabular}




\subsection{2.iii Dinucleotide occurrence in nucleosome DNA fragments}

Specific dinucleotides have previously been shown to be important for nucleosome positioning in many studies (Satchwell et al., 1986, Ioshikhes et al., 1996) in terms of dinucleotide frequency and position. The dinucleotides $[\mathrm{AA}],[\mathrm{TT}],[\mathrm{AT}]$ and [TA] have been shown to be important, with many nucleosome prediction algorithms based on patterns of distribution of these dinucleotides (Segal et al., 2006, Ioshikhes et al., 2006).

To test the hypothesis that Arabidopsis nucleosome positions are influenced by local sequence composition, dinucleotide observed/expected ratios were calculated for the Arabidopsis nucleosome DNA sequences for datasets wt_left and MET1_left. The expected values were calculated from the local nucleotide count and observed values were calculated from the local dinucleotide count (equation 3.3). The observed/expected dinucleotide ratios were also calculated for the whole Arabidopsis genome.

The mean observed/expected ratios of dinucleotide occurrence for each dataset are shown in Figure 3.9. There is little variation between the mean observed/expected ratios of the wt_left and MET1_mono nucleosome datasets. Both [AA] and [TT] dinucleotide frequencies occurred more often than expected (1.07 and 1.08 respectively) when compared to the local nucleotide count. However, when the nucleosome sequence distributions are compared to the whole genome, there appears to be very little variation. The dinucleotides $[\mathrm{CG}]$ and $[\mathrm{TA}]$ frequencies were both lower than expected ( 0.90 and 0.67 , respectively for wt_left, 0.91 and 0.68 , respectively for MET1_mono) when compared to the local nucleotide count, which is also observed in the whole genome. 

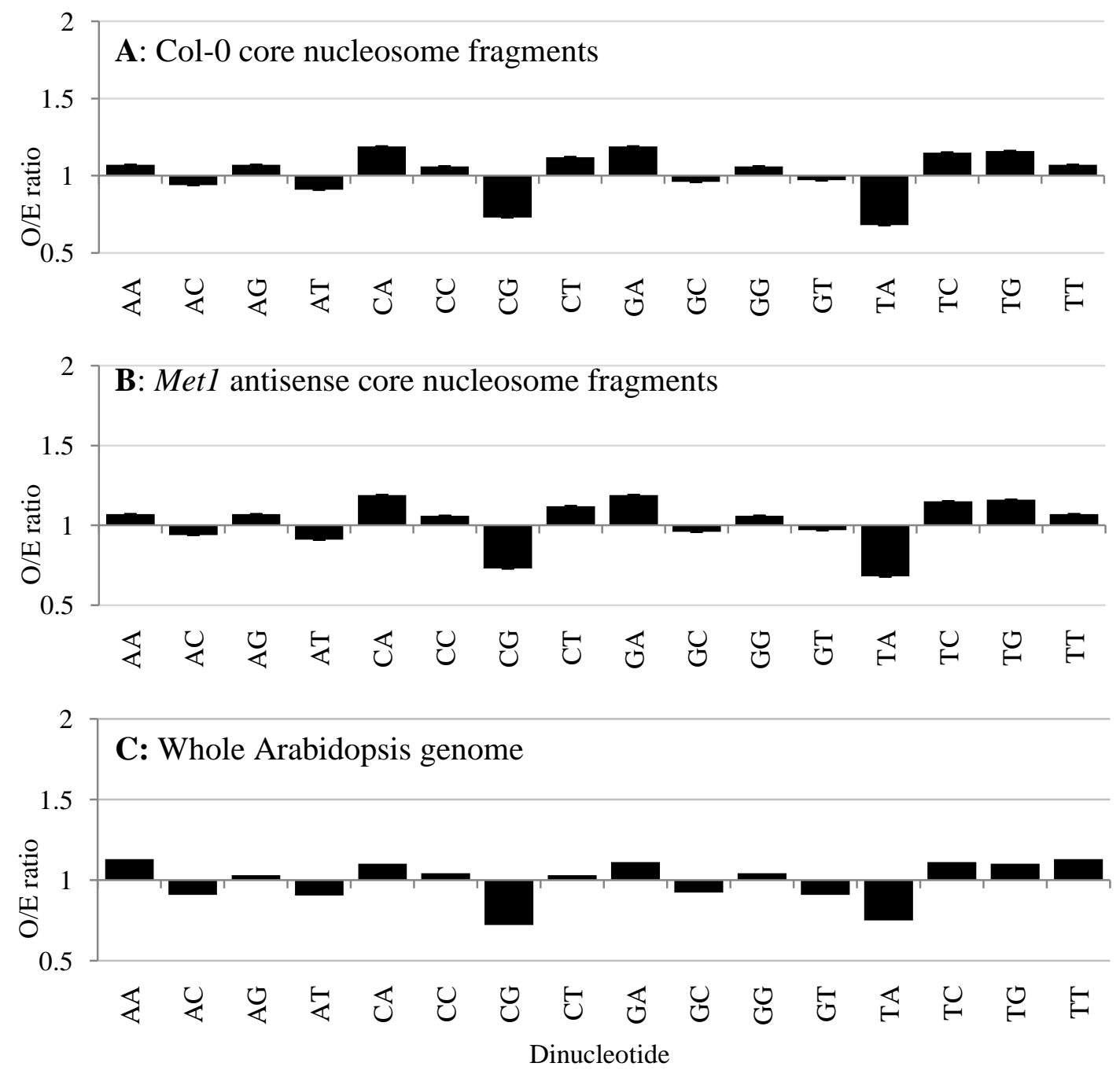

Figure 3.9 Mean observed/expected ratios of the occurrence of each dinucleotide for each DNA sequence in the nucleosome Arabidopsis datasets A: wt_left nucleosome DNA, B: MET1_mono nucleosome DNA, C: the whole genome. Expected values were calculated from the local DNA sequence composition. Error bars show standard error of the mean.

For comparison, the dinucleotide observed/expected ratios were also calculated for the linker DNA sequences from datasets wt_di-linker and MET1_di-linker, Figure 3.10. There appears to be slightly more variation in the occurrence of dinucleotides between the linker datasets than there is between the nucleosome datasets. Neither nucleosome nor linker datasets appear to show a strong bias towards a specific dinucleotide. 

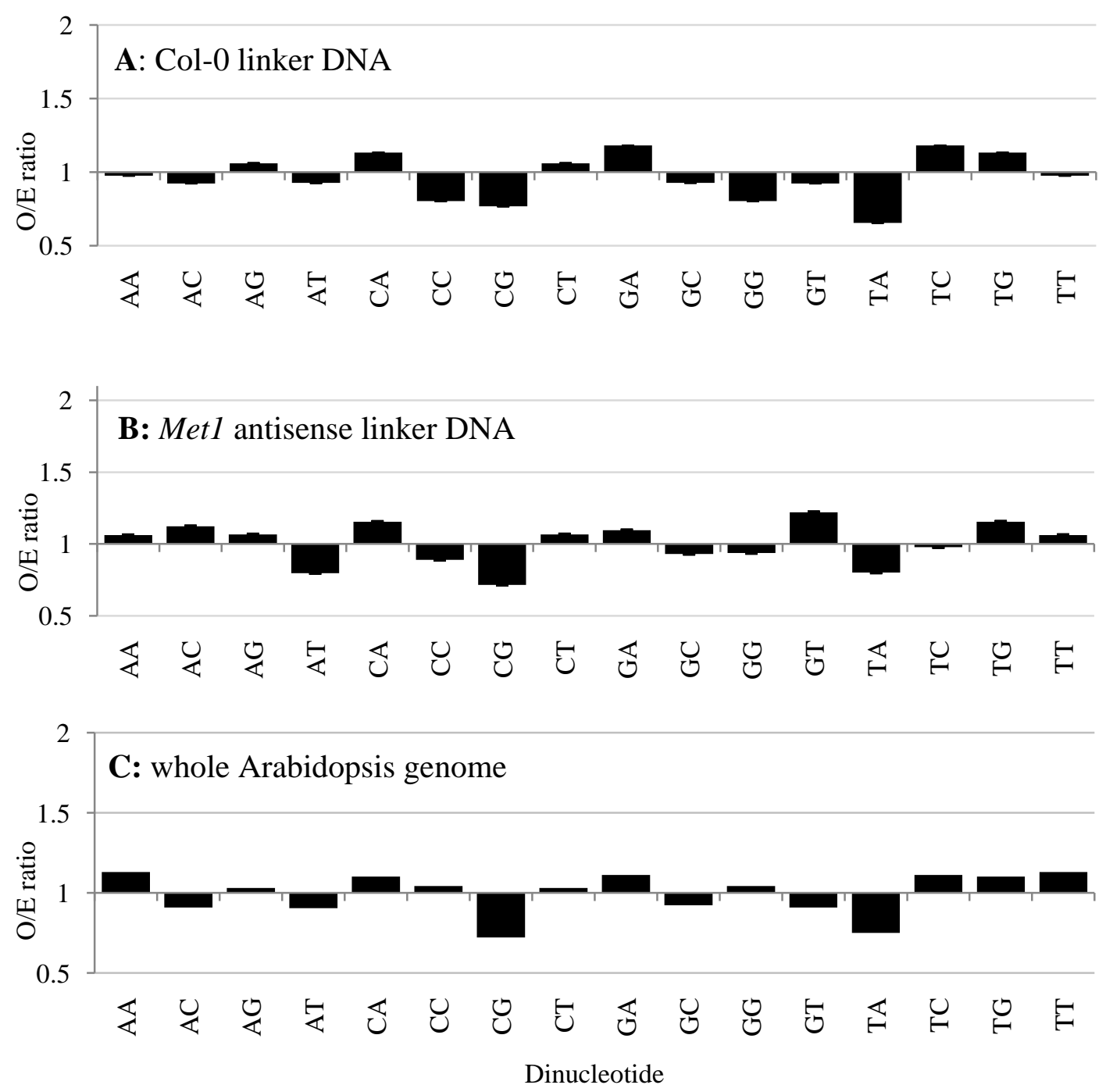

Figure 3.10 Mean observed/expected ratios of the occurrence of each dinucleotide for each DNA sequence in the linker Arabidopsis datasets A: wt_di-linker dataset, B: the MET1_di-linker dataset and $\mathbf{C}$ the whole genome. Expected values were calculated from the local DNA sequence composition. Error bars show standard error of the mean 


\subsection{2.iv Dinucleotide distribution within Arabidopsis nucleosome DNA fragments}

In many studies, the [AA/TT] dinucleotides have been found to occur at a higher frequency at preferred sites within the $147 \mathrm{bp}$ core nucleosome sequence, with a 10.3-10.4 bp periodic repeat, and reduced amplitude at the dyad axis (Ioshikhes et al., 1996).

In order to test the hypothesis that dinucleotides occur at preferred sites within Arabidopsis nucleosome sequences, the position of each [AA] and [TT] dinucleotide along the nucleosome core DNA fragment was determined for the $w t_{-} l e f t$ and MET1_mono datasets. The frequency at each position within the nucleosome core sequence was plotted and is shown in Figure 3.10.

A position for the dyad axis could be estimated by using the apparent symmetry of the $[\mathrm{AA} / \mathrm{TT}]$ distribution. This was placed at the mid-point between the first two peaks which are located at the edge of the region where the signal appears to be reduced in amplitude (Figure 3.10). This region is thought to indicate reduced bending of DNA at the nucleosome dyad axis (Ioshikhes et al., 1996). In these datasets, this position was detected $\sim 83 \mathrm{bp}$ in from the 5' edge of the nucleosome fragment, suggesting that during the digestion of chromatin, the MNase had not digested up to the nucleosome edge, since the mid-point of the nucleosome is thought to be around 73 bp (Richmond and Davey, 2003). This could either be due to the base-preference this enzyme exhibits, or because, for the present datasets, the enzyme had reduced access to the DNA, possibly because of the presence of histone H1 (An et al., 1998). 


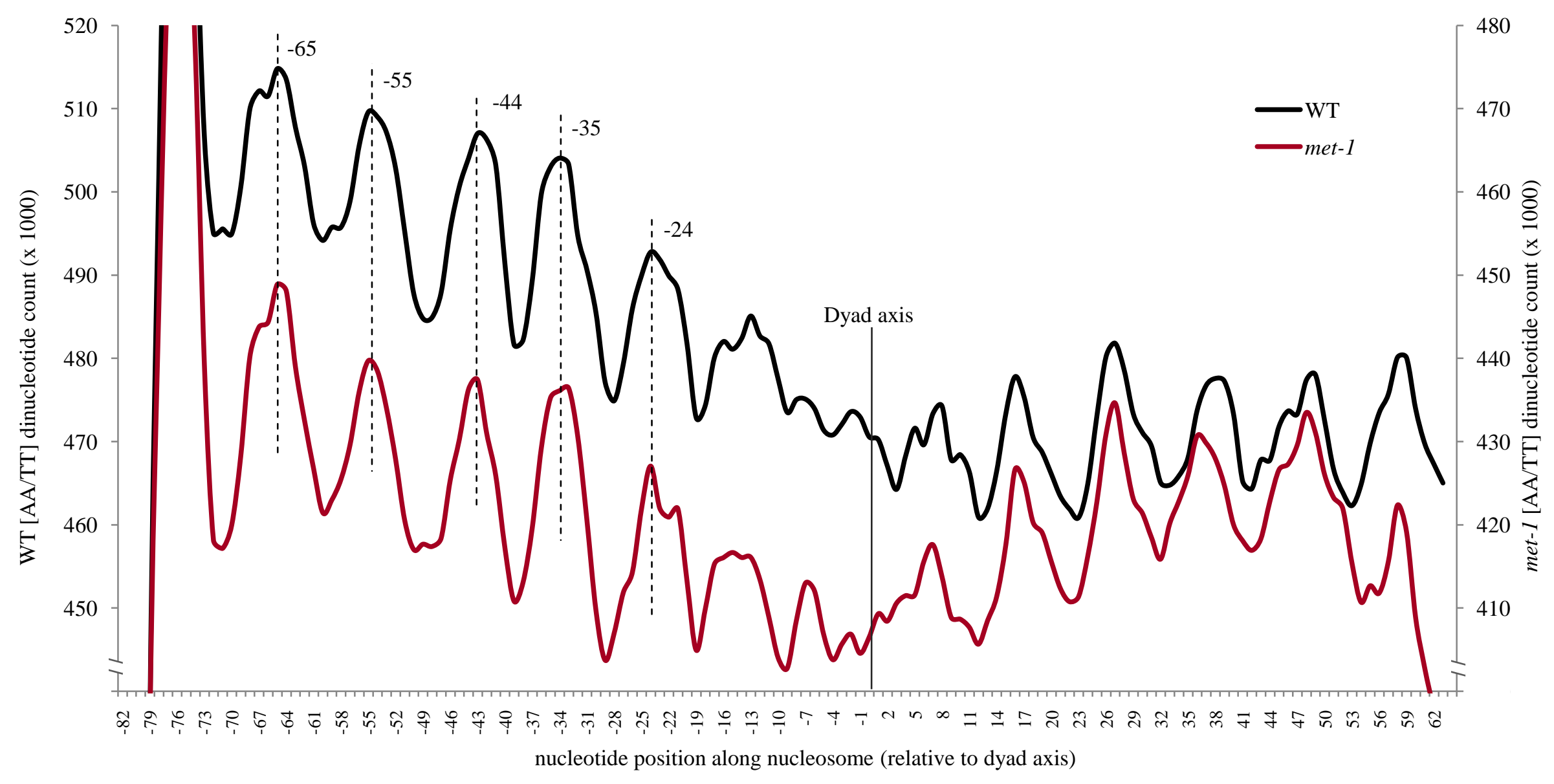

Figure 3.11 Distribution of [AA/TT] dinucleotides within nucleosome DNA fragments. Dataset $w t$ left is indicated with a black line ( $y$-axis on left) and dataset MET1_mono is indicated with a red line ( $y$-axis on right). The estimated dyad axis is indicated by a solid line, dashed lines indicate peaks in both datasets. 
Characteristics of nucleosome sequences

The $[\mathrm{AA} / \mathrm{TT}]$ dinucleotides displayed a non random distribution along the nucleosome DNA fragment. Peaks were observed at positions $-65,-55,-44,-35$, $-24,-13,-7,+7,+16,+27,+38,+48,+57$ relative to the dyad axis, with peaks displaying greater amplitude towards the 5' end of the nucleosome DNA fragment.

The positions of all [CC] and [GG] dinucleotides were determined for the nucleosome DNA fragments in datasets wt_left and MET1_mono, as shown in Figure 3.11. The distribution for [CC/GG] did not show the same periodicity as the $[\mathrm{AA} / \mathrm{TT}]$ distribution. However, peaks were observed in both the $w t$ left and MET1_mono datasets at $-73,-39$ and -5 , indicating that this dinucleotide may have a different role in positioning the DNA around the histone octomer. In particular, the peak at $-73 \mathrm{bp}$ suggests a bias for $[\mathrm{CC} / \mathrm{GG}]$ at the nucleosome/linker boundary, which has not previously been reported.

In order to test that the dinucleotide distributions observed could be attributed to nucleosome sequences, the distributions of $[\mathrm{AA} / \mathrm{TT}]$ and $[\mathrm{CC} / \mathrm{GG}]$ dinucleotides were calculated for the Ath_rand dataset (Figure 3.12). While peaks and troughs were observed in the distributions of both $[\mathrm{AA} / \mathrm{TT}]$ and $[\mathrm{CC} / \mathrm{GG}]$ dinucleotides, they appeared to be random. The distributions of dinucleotides within the random DNA sequences are dissimilar to the distributions of [AA/TT] dinucleotides in the nucleosome DNA sequences. Also, the distribution of [AA/TT] dinucleotides for the random DNA sequences do not display the apparent periodic distribution with an increase amplitude toward the 5' end observed in the distribution within the nucleosome DNA sequences. The peaks at specific nucleotide positions observed in the distribution of [CC/GG] dinucleotides for nucleosome sequences are also absent from the distribution for random DNA sequences. 


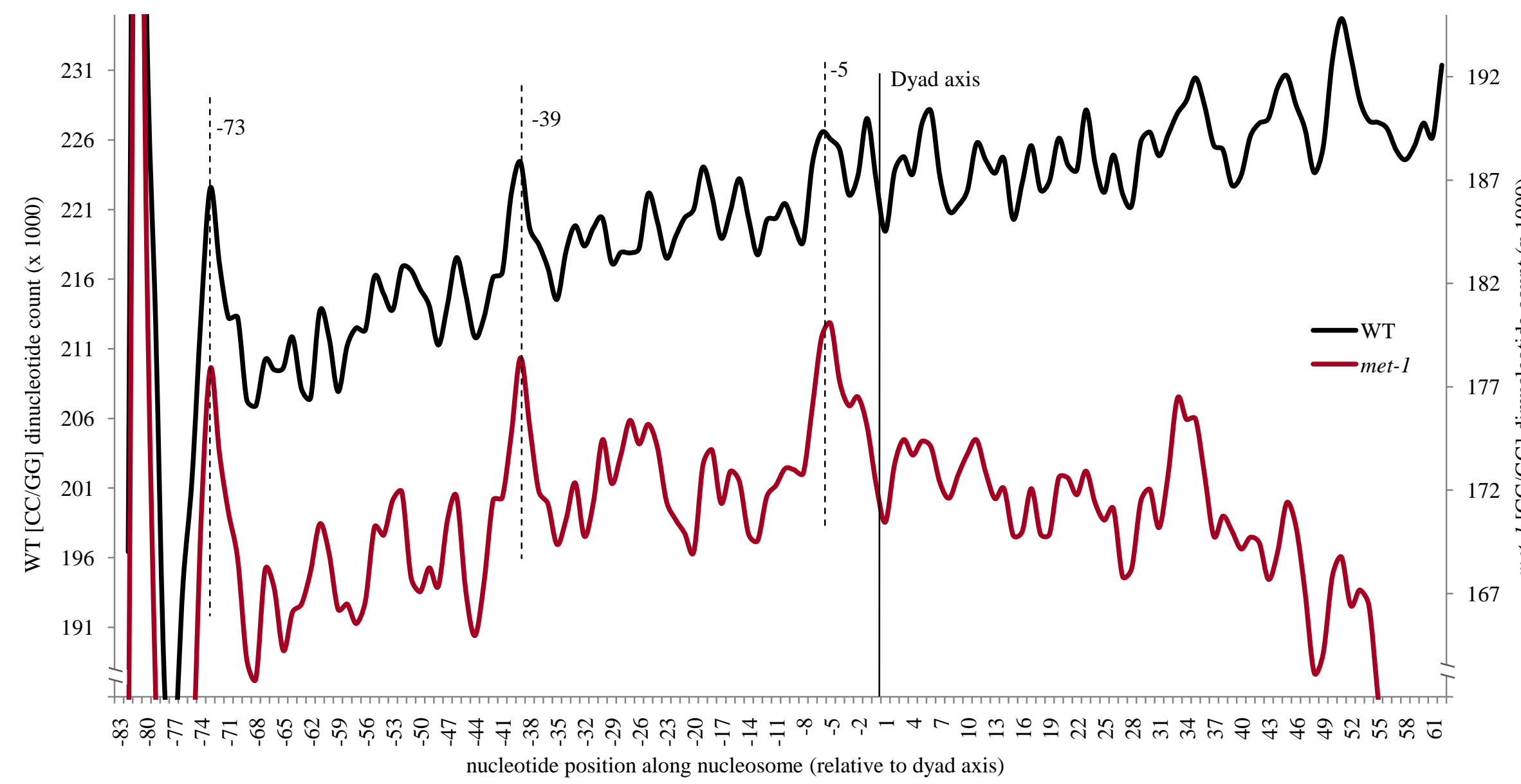




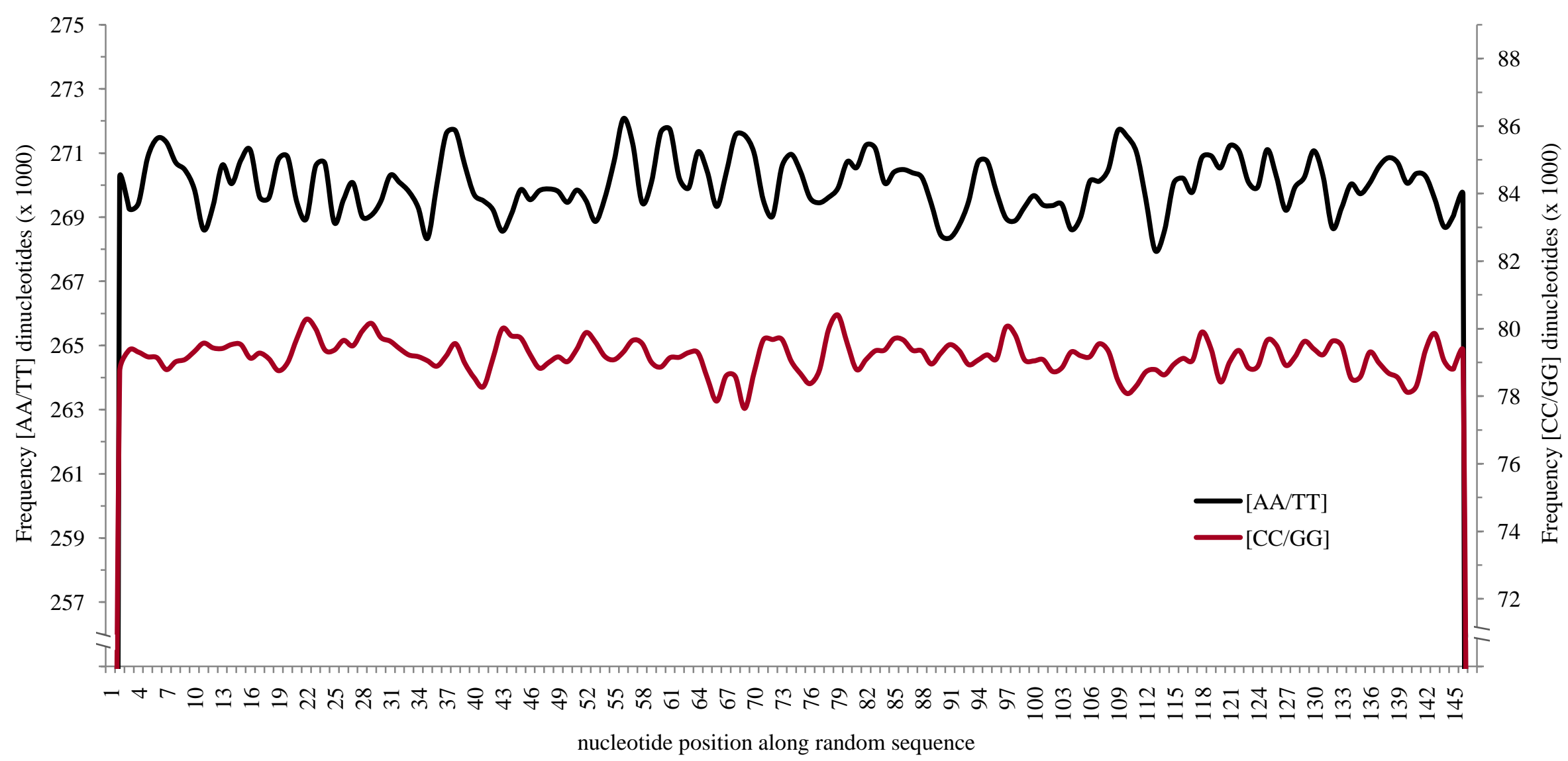

Figure 3.13 Distribution of [AA/TT] dinucleotides (black line) and [CC/GG] dinucleotides (red line) within the Ath_rand dataset. 
To test the hypothesis that nucleosomes form on DNA sequences which have patterns of dinucleotide distribution distinct from linker DNA sequences, the distributions of $[\mathrm{AA} / \mathrm{TT}]$ and $[\mathrm{CC} / \mathrm{GG}]$ were calculated for wt_left_linker and MET1_left_linker. The distributions, shown in Figure 3.13, indicate that differences are observed in the distributions of [AA/TT] dinucleotides between the nucleosome and linker DNA sequences. The apparently smooth, regular peaks of the nucleosome distributions are not observed in the linker distributions. This would be expected if the role of the linker DNA in chromatin is different from that of nucleosome DNA. In addition, some differences are observed in the distributions of [AA/TT] dinucleotides between wild-type and anti-sense MET1 linker DNA. In particular, the distributions within the wild-type and anti-sense MET1 from 0 to $\sim-35$ bp appear to be a mirror-image of each other (horizontally inverted at the $x$-axis). To test whether the apparent mirror-image in the distributions of [AA/TT] dinucleotides within the wt_left_linker and MET1_left_linker datasets was statistically significant, a test for autocorrelation was used to identify important peaks and troughs in the data. However, this failed to show any significant $(\mathrm{p}<0.05)$ peaks in either dataset. For example, the peak at $-10 \mathrm{bp}$ in the wild-type corresponds to a trough at this position in the anti-sense METI distribution. This could be due to an overall shift of nucleosome position in either dataset.

The distributions of the $[\mathrm{CC} / \mathrm{GG}]$ dinucleotides appear to be less organised than the [AA/TT] distribution in the wt_left_linker dataset. However, the distribution for MET1_left_linker appears to have a cyclical pattern with peaks at -2, -8, -15, -22, $30,-35,-42$ and $-50 \mathrm{bp}$. 

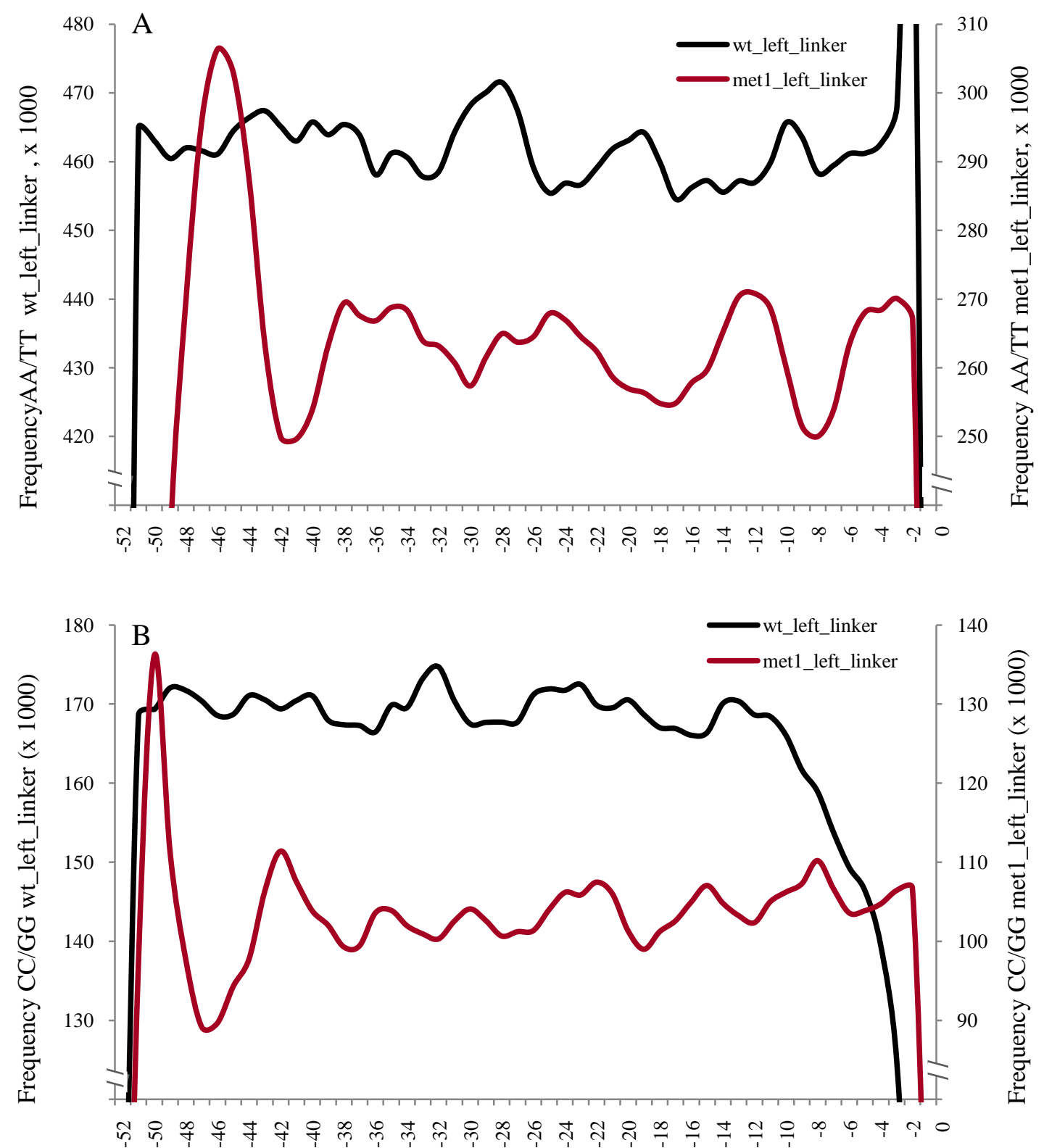

Distance from nucleosome (bp)

Figure 3.14 Distribution of dinucleotides along the linker DNA fragments of wt_left_linker and MET1_left_linker of A: [AA/TT] dinucleotides and B: [CC/GG] dinucleotides. The 5, edge of the nucleosome starts at position 0 on the right-hand side of each linker DNA fragment. 


\subsection{2.v Periodicity of dinucleotides in Arabidopsis nucleosome DNA fragments}

In order to test for periodicity in the distributions of dinucleotides along the nucleosome, Fourier analysis was performed on the frequencies of each complimentary pair of dinucleotides for the datasets wt_left, MET1_mono and Ath_02_wt. Prior to assessing periodicity, the distribution was twice-differenced to remove the overall trend in the data. The twice differenced [AA/TT] dinucleotide of the wt_left dataset is shown in Figure 3.14.

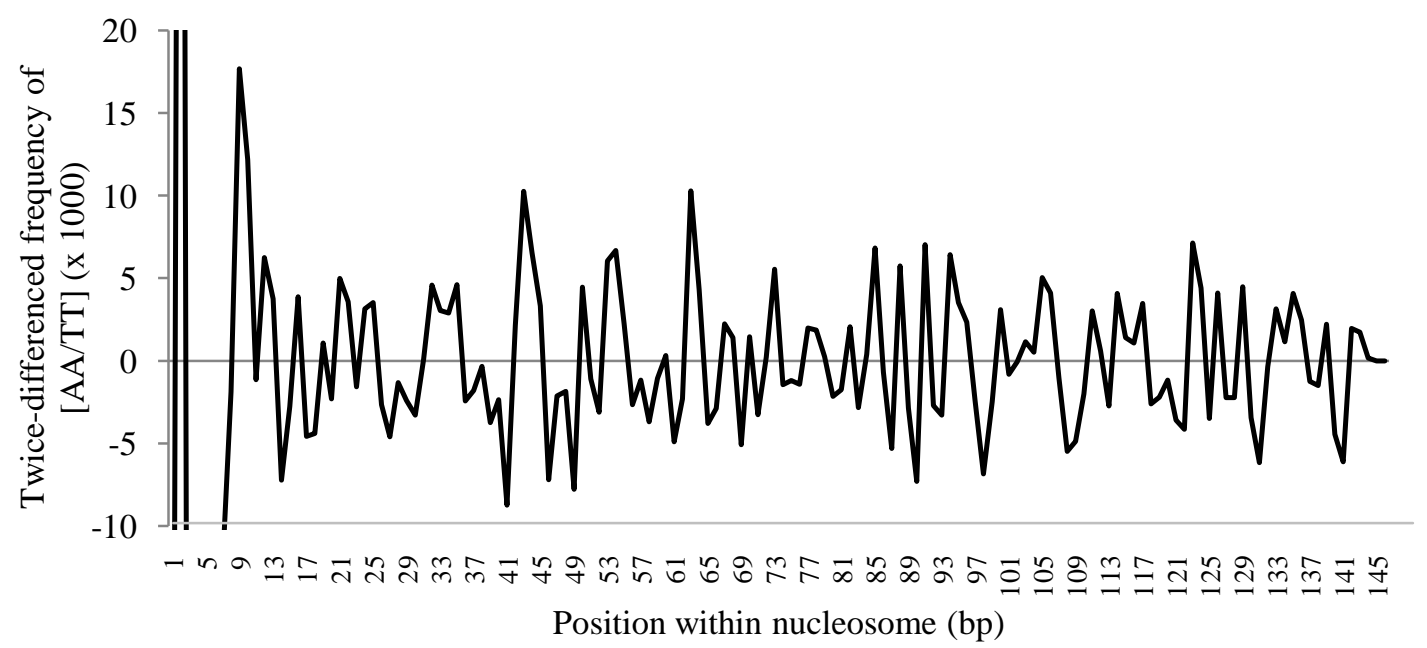

Figure 3.15 The twice-difference distribution of the [AA/TT] dinucleotide frequencies along nucleosome DNA fragments for the dataset $w t$ left.

To determine important periodicities in the distributions, Fourier-analysis was applied to the twice-differenced data and a periodogram was constructed. The periodogram is shown in Figure 3.15. The periodogram was inspected for statistically significant peaks, which indicate harmonics with important contributions to the variance in the data. The first statistically significant $(\mathrm{p}<0.05)$ peak at amplitude of 0.0966 corresponds to a periodicity of $10.35 \mathrm{bp}$ in the distribution of [AA/TT] dinucleotides. Table 3.7 shows the periodicities calculated by Fourier analysis for datasets $w t \_l e f t, M E T 1 \_m o n o, A t h \_02 \_w t$ and the periodicity of [AA/TT] and $[\mathrm{CC} / \mathrm{GG}]$ in the $A t h \_r a n d$ dataset. 


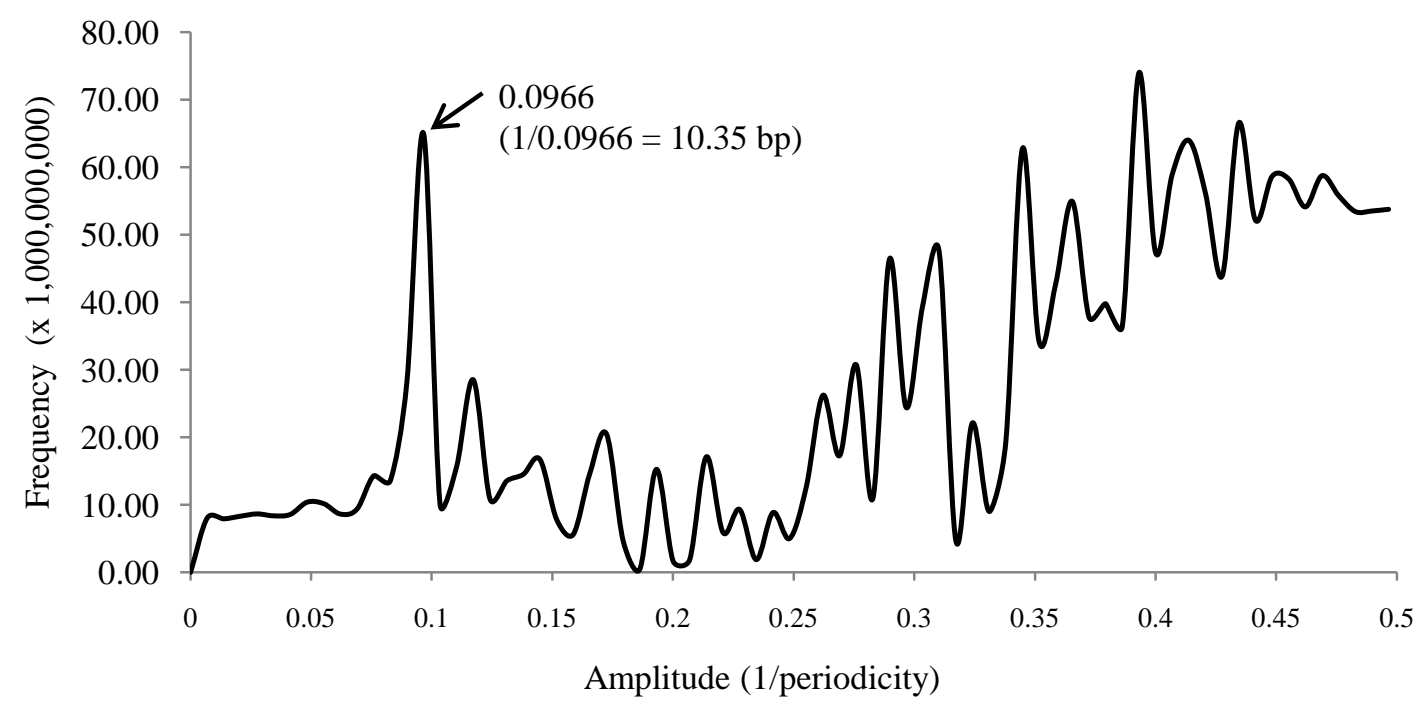

Figure 3.16 Periodogram constructed by Fourier analysis of the [AA/TT] dinucleotide distribution for the wt_left dataset. The arrow indicates the first important harmonic in the periodogram, indicating a periodicity of $10.35 \mathrm{bp}$ for [AA/TT] dinucleotides.

Table 3.7 Statistically significant periodicities calculated by Fourier analysis for each complementary pair of dinucleotides for the nucleosome datasets wt_left, MET1_mono, Ath_02_wt and Ath_rand. The largest statistically significant $(\mathrm{p}<0.05)$ periodicity is shown in each case.

\begin{tabular}{l|rrrr}
\hline \multirow{2}{*}{ Dinucleotide pair } & \multicolumn{4}{|c}{ Periodicity (bp) } \\
& wt_left & MET1_mono & Ath_02_wt & Ath_rand \\
\hline AA/TT & 10.35 & $20.71^{*}$ & 10.35 & 12.08 \\
AC/GT & 10.35 & $13.18^{*}$ & $20.71^{*}$ & \\
AG/CT & 6.31 & 10.35 & 10.35 & \\
AT/TA & 10.35 & 7.63 & 10.35 & \\
CA/TG & 6.19 & 13.18 & 10.35 & \\
CG/GC & $28.99^{*}$ & $28.99^{*}$ & 18.11 & \\
CC/GG & 13.18 & 13.18 & 8.53 & 24.15 \\
GA/TC & $13.18^{*}$ & $13.18^{*}$ & 6.19 & \\
\hline
\end{tabular}

* These analyses also show evidence of periodicity of $10.35 \mathrm{bp}$

The dinucleotide $[\mathrm{AA} / \mathrm{TT}]$ has a periodicity of $10.35 \mathrm{bp}$ for both the $w t_{-}$left and Ath_O2_wt datasets, while the MET1_mono shows an important periodicity at 20.71 bp. However, for this dataset, there was also a significant harmonic relating to 10.35 , suggesting that there may be preferred positions occurring more often in the MET1_mono dataset than in the others. The $[\mathrm{CC} / \mathrm{GG}]$ dinucleotide periodicity was calculated to be 13.18 bp in the wt_left and MET1_mono datasets and $8.53 \mathrm{bp}$ for the Ath_02_wt dataset. 
There appears to be a difference in the periodicity of the [AC/GT] dinucleotide between anti-sense MET1 and both (wt_left and Ath_O2_wt) wild-type datasets. For the [AT/TA] dinucleotide, both wild-type datasets show a periodicity of $10.35 \mathrm{bp}$, while the anti-sense MET1 has a periodicity of $7.63 \mathrm{bp}$. This suggests differences in the contribution of dinucleotides to the nucleosome positioning signal between antisense MET1 and wild-type.

The $[\mathrm{CA} / \mathrm{TG}]$ dinucleotide shows a different periodicity for each dataset. It is possible that this dinucleotide does not contribute to the overall nucleosome positioning signal. However, it could be the case (as with all dinucleotides) that this dinucleotide contributes to a specific set of nucleosome positions, for example, alternative positions around TSSs. Several differences are observed between Ath_02_wt and the other two sets. The random DNA sequences show periodicity in the distribution of $[\mathrm{AA} / \mathrm{TT}]$ of $12.08 \mathrm{bp}$ and in the distribution of [CC/GG] dinucleotides of $24.15 \mathrm{bp}$. The periodicities of the [AA/TT] and [CC/GG] distributions were also calculated for the wt_left_linker and MET1_left_linker as for the nucleosome datasets. These are shown in Table 3.8. The [CC/GG] dinucleotide occurred with a 10.20 bp periodicity in both the wt_left_linker and the MET1_left_linker datasets, although there was also evidence of a 7.28 bp periodicity for $[\mathrm{CC} / \mathrm{GG}]$ in the anti-sense $M E T 1$ linker.

Table 3.8 The periodicities of the linker datasets: $w t$ left_linker and MET1_left_linker

\begin{tabular}{|c|c|c|}
\hline \multirow[t]{2}{*}{ Dinucleotide pair } & \multicolumn{2}{|c|}{$\overline{\text { Periodicity }(\mathrm{bp})}$} \\
\hline & $\begin{array}{l}\text { wt_left } \\
\text { _linker }\end{array}$ & $\begin{array}{l}\text { MET1_left } \\
\text { linker }\end{array}$ \\
\hline $\mathrm{AA} / \mathrm{TT}$ & 4.64 & 7.28 \\
\hline $\mathrm{CC} / \mathrm{GG}$ & 10.20 & 10.20 \\
\hline
\end{tabular}

Both the wild-type and anti-sense MET1 datasets show differences between the nucleosome DNA and the linker DNA in terms of the periodicity of [AA/TT] dinucleotides. 


\subsection{3.i Nucleosome Sequence database}

The construction of the Nucleosome Sequence database was initiated in 2006, when the amount of available nucleosome positioning data was small and not collated into a single resource. The Nucleosome Sequence database was populated with data from a number of sources with the aim of producing a single source for all the publicly available datasets at the time. The database contains nucleosome position information on species shown in Table 3.9.

Table 3.9 A list of the species represented in the Nucleosome Sequence database, collected from a variety of sources, and the number of entries for each species.

\begin{tabular}{|c|c|c|}
\hline Species & $\begin{array}{l}\text { Number } \\
\text { of } \\
\text { entries }\end{array}$ & Data source \\
\hline Bovine spp & 7 & Ioshikhes et al. (1996) \\
\hline Chlorocebus spp & 1 & Ioshikhes et al. (1996) \\
\hline Cricetulus spp & 1 & Ioshikhes et al. (1996) \\
\hline Crithidia fasciculata & 1 & Ioshikhes et al. (1996) \\
\hline Drosophila melanogaster & 32 & $\begin{array}{l}\text { Ioshikhes et al. (1996), NPRD: Levitsky et } \\
\text { al. (2005) }\end{array}$ \\
\hline Euplotes eurystomus & 4 & Ioshikhes et al. (1996) \\
\hline Gallus spp* & 338 & $\begin{array}{l}\text { A. Travers. (Drew and Travers (1986), } \\
\text { Satchwell and Travers (1989)), Ioshikhes et } \\
\text { al. (1996) }\end{array}$ \\
\hline Homo sapiens & 18 & $\begin{array}{l}\text { Ioshikhes et al. (1996), NPRD: Levitsky et } \\
\text { al. (2005) }\end{array}$ \\
\hline Human immunodeficiency virus type 1 & 6 & Ioshikhes et al. (1996) \\
\hline Human papillomavirus type 16 & 2 & NPRD: Levitsky et al. (2005) \\
\hline Lytechinus variegatus & 1 & Ioshikhes et al. (1996) \\
\hline Mouse mammary tumour virus & 6 & Ioshikhes et al. (1996) \\
\hline Mus musculus & 8 & $\begin{array}{l}\text { Ioshikhes et al. (1996), NPRD: Levitsky et } \\
\text { al. (2005) }\end{array}$ \\
\hline Oxytricha nova & 3 & Ioshikhes et al. (1996) \\
\hline Psammechinus miliaris & 1 & Ioshikhes et al. (1996) \\
\hline Rattus norvegicus & 12 & $\begin{array}{l}\text { Ioshikhes et al. (1996), NPRD: Levitsky et } \\
\text { al. (2005) }\end{array}$ \\
\hline Saccharomyces cerevisiae & 275 & $\begin{array}{l}\text { Ioshikhes et al. (1996), NPRD: Levitsky et } \\
\text { al. (2005) }\end{array}$ \\
\hline Schizosaccharomyces pombe & 12 & Ioshikhes et al. (1996) \\
\hline Simian virus 40 & 53 & $\begin{array}{l}\text { Ioshikhes et al. (1996), NPRD: Levitsky et } \\
\text { al. (2005) }\end{array}$ \\
\hline Tetrahymena thermophila & 11 & Ioshikhes et al. (1996) \\
\hline Xenopus laevis & 5 & Ioshikhes et al. (1996) \\
\hline Zea mays & 2 & Ioshikhes et al. (1996) \\
\hline
\end{tabular}


Characteristics of nucleosome sequences

Datasets generated since 2005 utilising new sequencing technologies and highresolution tiling arrays has vastly increased the amount of nucleosome positioning data available. From this time, many datasets contribute to give insight into the distribution of nucleosome positions across whole genomes (Mavrich et al., 2008a, Zhang et al., 2008, Albert et al., 2007, Johnson et al., 2006, Lee et al., 2007, Yuan et $a l$. , 2005). At the time of writing, these sets had not been added to the original database, but those that were available are included here as a collection of nucleosome positions for comparisons and analysis. These are summarised in Table 3.10 .

Table 3.10 A summary of some of the large nucleosome position datasets generated by highthroughput, next generation technologies, which were available, at the time of writing, for data comparisons and analysis.

\begin{tabular}{|c|c|c|c|c|}
\hline Species & Nucleosome class & Technology & $\begin{array}{l}\text { Number of } \\
\text { entries }\end{array}$ & Reference \\
\hline H. sapiens & dinucleosome & $\begin{array}{l}\text { Traditional Sanger } \\
\text { sequencing }\end{array}$ & 1,002 & Kato et al., 2003 \\
\hline H. sapiens & dinucleosome & $\begin{array}{l}\text { Traditional Sanger } \\
\text { sequencing }\end{array}$ & 100 & Kato et al., 2005 \\
\hline C. elegans & mononucleosome & $\begin{array}{l}454 \\
\text { (pyrosequencing) }\end{array}$ & $\begin{array}{r}187,863 \\
\text { (aligned) }\end{array}$ & Johnson et al., 2006 \\
\hline D. melanogaster & $\begin{array}{l}\text { mononucleosome } \\
\text { (H2AZ-containing) }\end{array}$ & $\begin{array}{l}\text { GS20FLX and } \\
\text { tiling array }\end{array}$ & $\begin{array}{r}207,025 \\
\text { (aligned) }\end{array}$ & Mavrich et al., 2008a \\
\hline S. cerevisiae & mononucleosome & $\begin{array}{l}\text { Traditional Sanger } \\
\text { sequencing }\end{array}$ & 199 & Segal et al., 2006 \\
\hline S. cerevisiae & $\begin{array}{l}\text { mononucleosome } \\
\text { (H2AZ-containing) }\end{array}$ & Pyrosequencing & 322,000 & Albert et al., 2007 \\
\hline S. cerevisiae & mononucleosome & Tiling array & 2,278 & Yuan et al., 2005 \\
\hline
\end{tabular}




\subsection{Discussion}

\subsubsection{Micrococcal nuclease sequence preferences}

Sequence preferences at the site of MNase activity have been reported previously. A study to determine sequence preferences undertaken by Dingwall and colleagues (Dingwall et al., 1981) showed a preference for [WG] in bovine DNA. In addition, a preference for [WG] has also been demonstrated at the site of MNase activity in yeast (Segal et al., 2006). In contrast, human nucleosome DNA did not show a [WG] bias, although a preference for [GG] dinucleotides was observed. This led to speculation that the MNase preference might form part of a nucleosome positioning signal outside the nucleosome core boundary (Kogan et al., 2006). However, in these studies, nucleotide preferences at the site of MNase activity were based on counts of occurrences, and did not take into consideration the frequency of dinucleotides in the genome from which the chromatin was prepared. Comparisons of observed/expected ratios calculated in this study give a more accurate picture of the effect of MNase preference on digested chromatin.

The Arabidopsis observed/expected ratios for MNase nucleotide preference show the bias towards [WG] similar to what may be expected given those identified by Dingwall et al., (1981). Observed/expected ratios calculated for other taxa showed that different nucleotides occurred at frequencies greater than expected when compared to the respective genomic ratios. In the human datasets, the preferences for $[\mathrm{CG}]$ were 11-15 times higher than expected when compared to the genome occurrence of [CG]. A preference for [CG] was also observed in untrimmed chicken nucleosomes, eight times more often than expected when compared to the whole genome. The reported MNase preference for [WG] is observed in all datasets analysed with the exception of the trimmed chicken dataset. However, the observed occurrence of other dinucleotides at the sites of MNase action suggests that the MNase preference may be dependent on other factors such as the local chromatin structure. This could either be due to the presence of histone H1, or steric hindrance preventing MNase access to closely-spaced nucleosomes. 
It is unlikely that any sequence-specificity which MNase possesses is adversely affecting the analysis of sequence composition in this study. The distribution of dinucleotides within the nucleosome sequence (section 3.3.2.iv) shows a possible 10 bp gap between the edge of the DNA fragment and the start of the nucleosome, according to the estimate of the dyad axis. The presence of histone H1 is thought to protect 5-15 bp linker DNA from MNase digestion (An et al., 1998) and could be the cause of the gap, alternatively it could be incomplete digestion of the linker to the nucleosome edge. Arabidopsis chromatin was formaldehyde cross-linked prior to MNase digestion. This should ensure that nucleosomes do not alter their translational position during the preparation to provide fragments and so should preserve the in vivo nucleosome positions as well as the positioning signals associated with them.

Another apoptotic enzyme, DNA fragmentation factor 40/caspase-activated deoxyribonuclease (DFF40/CAD) has been developed for the purpose of studying chromatin structure and is reportedly superior to MNase in its specificity for linker DNA as a substrate (Widlak and Garrard, 2006). However, this enzyme is also reported to have a stronger sequence preference than MNase and shows a bias towards sequences which contain [RRRYRYYY], cleaving between the $4^{\text {th }}$ and $5^{\text {th }}$ position.

\subsubsection{Nucleosome DNA fragment GC content}

The sequence composition of nucleosome DNA fragments was compared to the genome average with respect to GC content. The nucleosome DNA fragments had a considerably higher GC content than the average, and when compared to subsets of the Arabidopsis genome, the mean GC of nucleosome sequences (41\% GC) was between that associated with exons (42\%) and the 5'UTRs (38\%). This may suggest that the nucleosome datasets are enriched for exonic sequences. A previous computationally-based study places nucleosome positioning signals at intron/exon boundaries (Kogan and Trifonov, 2005). If this is the case, the GC content of the nucleosome sequences would suggest that the nucleosome occupies the 'exonic side' of the boundary, as the GC content for nucleosome sequences is well above that for introns ( $33 \%$ GC). It could be the case that exonic nucleosomes have more defined 
Characteristics of nucleosome sequences positions compared to intronic sequences, which may be less structured. This may result in exonic sequences being represented more often in nucleosome datasets.

\section{$\underline{\text { 3.4.3 Nucleosome DNA fragment dinucleotide occurrence }}$}

The observed/expected ratios of dinucleotide occurrence within nucleosome DNA fragments showed that there was little deviation from the expected values when compared to the local nucleotide count. Occurrences of $[\mathrm{AA}],[\mathrm{TT}]$ and $[\mathrm{CA}]$ were higher than expected. However, the occurrence of the $[\mathrm{AA}]$ and $[\mathrm{TT}]$ dinucleotides appear to be higher in the Arabidopsis genome when compared to the nucleosome sequences. These dinucleotides have all been implicated in positioning nucleosomes in previous studies (Widlund et al., 1997, Ioshikhes et al., 1996). The wild-type linker dataset (wt_di-linker) shows slightly more variation between the datasets when compared to the nucleosome dataset. It is likely that the positioning of specific dinucleotides is more important for nucleosome positioning than the overall abundance, and therefore, those dinucleotides are not likely to occur in nucleosome sequences more often than expected.

\subsubsection{Distributions of dinucleotides within nucleosome DNA}

The characteristic distribution of the dinucleotides [AA/TT] within the nucleosome DNA fragments observed in this study has been observed previously (Ioshikhes et al., 1996). The in-phase $10 \mathrm{bp}$ periodic repeat of [AA/TT] suggests a curved structure in the DNA (Satchwell et al., 1986). The distribution of [AA/TT] dinucleotides within Arabidopsis nucleosome DNA fragments has a periodicity of $10.35 \mathrm{bp}$, so this is further evidence for sequence-dependent DNA bending around the nucleosome.

The dinucleotides [CC] and [GG] are thought to have a similar distribution along the nucleosome DNA as [AA] and [TT], but occur a half-turn (5 bp) out of phase with the position of [AA/TT] (Ioshikhes et al., 1996). The [CC/GG] dinucleotide distribution did not show the expected counter-phase distribution to that of the [AA/TT] in Arabidopsis. The [CC/GG] distribution shows peaks at specific points 
along the nucleosome in both the wild-type and anti-sense MET1 datasets. This suggests a different role for $[\mathrm{CC} / \mathrm{GG}]$ from that of $[\mathrm{AA} / \mathrm{TT}]$ in nucleosome positioning. A periodicity of $13.18 \mathrm{bp}$ in the distribution of [CC/GG] dinucleotides further supports this hypothesis.

Differences in the periodicity of [AC/GT] dinucleotides were observed between antisense MET1 and both wild-type datasets. This may suggest differences in the contribution of dinucleotides to the nucleosome positioning signal between antisense METI and wild-type. This is of interest since this dinucleotide has been shown to contribute to the nucleosome positioning signal (Segal et al., 2006, Widlund et al., 1997). Several differences were observed between Ath_02_wt and the Ath_03_wt datasets. This could be due to subtle differences in environmental growing conditions experienced by the plants on the different occasions the nucleosome fragments were isolated.

Of particular interest is the distribution of dinucleotides within the derived linker sequences. The wild-type and anti-sense MET1 datasets show an almost mirrorimage in $[\mathrm{AA} / \mathrm{TT}]$ distribution, but appear to have different periodicities within the distribution. This adds further evidence to the hypothesis that nucleosome occupancy in anti-sense MET1 is different from wild-type. Investigation of autocorrelation did not identify important peaks and troughs in the wild-type and anti-sense METI dinucleotide distributions. However, inspection of the difference between peaks in the distribution of [AA/TT] when comparing the wild-type and anti-sense MET1 linkers suggests that there may be a difference between the two datasets of approximately five to eight bp (or multiples of five to eight bp).

The periodicities in the distribution of $[\mathrm{AA} / \mathrm{TT}]$ dinucleotides along the linkers of wild-type and anti-sense MET1 are different, with the wild type linker distribution appearing to have many small peaks which are absent from the MET1_left_linker distribution. This may account for the difference in periodicities. The periodic distribution of $[\mathrm{AA} / \mathrm{TT}]$ dinucleotides within nucleosome DNA is thought to extend to approximately $200 \mathrm{bp} \mathrm{(} \sim 50 \mathrm{bp}$ beyond the edge of the nucleosome) (Ioshikhes et $a l ., 1996)$ and represents alternative nucleosome positions. This was not observed in Arabidopsis. However, the distributions of $[\mathrm{CC} / \mathrm{GG}]$ dinucleotides in the left linkers 
of the wild-type and anti-sense MET1 datasets did show a periodicity of $10.20 \mathrm{bp}$. This periodicity was not observed in the nucleosome DNA sequences, suggesting that $[\mathrm{CC} / \mathrm{GG}]$ dinucleotides have different roles for nucleosome positioning in nucleosome and linker DNA, for example, $[\mathrm{CC} / \mathrm{GG}]$ dinucleotides may exclude nucleosomes in some contexts. This phenomenon has not been previously reported. In general, there appears to be little information regarding the sequence characteristics of linker DNA compared to nucleosome DNA in the literature.

The differences observed for dinucleotide occurrence and distribution between wildtype and anti-sense METI datasets may reflect differences in chromatin structure brought about by DNA methylation (or the absence of DNA methylation). Previous studies have shown that DNA methylation can alter the position of a nucleosome in vitro. Reconstitution of nucleosomes onto methylated and unmethylated versions of the chicken $\beta$-globin gene promoter demonstrated that the presence of DNA methylation altered nucleosome occupancy at this site (Davey et al., 1997). When DNA methylation occurred near to the dyad axis of a nucleosome (1.5 helical turns from the dyad axis), nucleosome occupancy was dramatically reduced. DNA methylation at other positions had little or no effect. Further studies demonstrated that DNA methylation of a prokaryotic DNA sequence (E. coli tyr T promoter) altered the preferred rotational positioning of nucleosomes (Buttinelli et al., 1998). Addition of 5-methylcytosine groups to the major grooves shifted the nucleosome position three bases towards the 3' end of DNA fragments. This suggests that presence of 5-methylcytosine groups on nucleosome DNA may affect the bending of DNA around the histone core by altering the base-stacking parameters of the dinucleotides.

\subsubsection{Summary}

The characteristics of nucleosome sequences in Arabidopsis show some similarities to those isolated in some other genera, such as higher GC content compared to the genomic average (Ozsolak et al., 2007), the occurrence of [A] and [T] at the site of MNase activity, and the distribution of $[\mathrm{AA} / \mathrm{TT}]$ dinucleotides with a periodicity of 10.35 bp (Segal et al., 2006; Ioshikhes et al., 1996; Satchwell et al., 1986). In contrast, Arabidopsis nucleosome sequences also exhibit distinct patterns of 
Characteristics of nucleosome sequences [CC/GG] dinucleotide distribution within nucleosome sequences and differences in the distribution of dinucleotides between the nucleosome and linker DNA. Particularly interesting are the differences revealed in sequence preferences for nucleosome and linker DNA between wild-type and anti-sense MET1. These differences suggest a role for DNA methylation in nucleosome positioning (see Chapter 5). 
Chapter 4

Nucleosome spacing and linker length variation 


\subsection{Introduction}

\section{$\underline{4.1 \text { Introduction to linker DNA and linker length variation }}$}

Linker DNA is defined as the DNA connecting two nucleosomes. Linker histones (H1/H5) anchor the nucleosome in place and contribute to the compaction of the chromatin fibre by interacting with DNA, both in the nucleosome core and in the linker region (Fan et al., 2003).

Early studies of the structure of chromatin involved the visualisation of chromatin by electron microscopy (Olins and Olins, 1974, Finch et al., 1975, Oudet et al., 1975). These studies revealed chromatin to be a heterogeneous molecule composed of repeating units of 'spherical bodies' (or nucleosomes) connected by 'DNA filaments' (linker DNA). These experiments provided the first evidence that chromatin structure is conserved between species, but that the linker length and level of chromatin compaction is variable.

Differences in DNA linker length were also noticed by Lohr and colleagues (Lohr et al., 1977). Staphylococcal nuclease digestion of chicken erythrocyte, yeast and HeLa chromatin revealed differences in the nucleosome repeat length (the distance between the centres of two adjacent nucleosomes) but not in the DNA wrapped around the core particle. This suggested that the variability was due to the differences in linker length between these species. The differences in linker lengths between the three species included in the study were attributed to the transcriptional activity of the cell-type from which the chromatin originated. The linkers were found to be more variable in the HeLa and yeast chromatin, which is transcriptionally active, whereas the relatively inactive chicken erythrocyte chromatin was found to have regularly ordered nucleosome spacing. Further study of chicken erythrocyte, yeast and HeLa chromatin led to the observation of a linker length phasing in chromatin of $10_{n}$ (or multiples of the helical repeat of DNA). In yeast chromatin the linker length phasing was $10_{n}+5$ bp which suggested that nucleosomes in yeast chromatin are positioned in an anti-parallel manner with respect to the adjacent nucleosome (Lohr et al., 1979). 
Nucleosome spacing and linker length variation Further evidence for the relationship between transcriptional activity and linker length was provided by experiments determining the linker length of the developing chicken from embryo to adult (Weintraub, 1978). The nucleosome repeat length of a 4-day old chick embryo was shown to be $190 \mathrm{bp}$ (equivalent to a linker length of 43 bp). This increased to a nucleosome repeat length of 212 bp (equivalent to a linker length of $65 \mathrm{bp}$ ) in the adult chicken. The increase in nucleosome repeat length correlated with an increase in the number of molecules of erythrocyte-specific histone H5 from 0.2 molecules $\mathrm{H} 5 /$ nucleosome in the embryo to 1 molecule $\mathrm{H} 5 /$ nucleosome in the adult.

The nucleosome repeat lengths from many studies were collated and published in a taxa-organised list containing 185 entries (van-Holde, 1989). These data were used to determine if nucleosome repeat lengths do indeed show the multiples of preferential lengths that had been suggested previously (Widom, 1992). Firstly, probability distribution functions were constructed for average nucleosome repeat lengths. These were reported with error values, and the probability distributions were investigated using Fourier analysis. This revealed a periodicity of 9 to $10 \mathrm{bp}$ within these data which is consistent with the periodicity of the helical repeat of DNA. This result disagreed with an earlier study where chromatin was reconstituted onto salmon sperm DNA and the psf 2124 plasmid without the presence of a linker histone. Micrococcal nuclease digestion and investigation by electron microscopy revealed that these nucleosomes, when closely spaced, appeared to have a preferred linker length of multiples of $7 \mathrm{bp}$ (Noll et al., 1980). However, since then, an approximate $10 \mathrm{bp}$ periodicity has been demonstrated in the distribution of linker lengths in yeast chromatin (Wang et al., 2008; Cohanim et al., 2005) and in the predicted linkers of human chromatin (Kato et al., 2003).

Linker length is thought to be a major determinant of higher-order chromatin structure, and specifically contributes to the diameter of the ' $30 \mathrm{~nm}$ ' fibre (Robinson, et al., 2007, Wong et al., 2007). Measurements of chromatin fibres reconstituted from ordered nucleosome arrays revealed two classes. The first has a diameter $\sim 35$ $\mathrm{nm}$ for nucleosome repeat lengths of 177 to $207 \mathrm{bp}$ (equivalent to linker length of 30 to $60 \mathrm{bp}$ ). The second class of fibre constructed from nucleosome arrays with repeat 
Nucleosome spacing and linker length variation lengths of 217 to $237 \mathrm{bp}$ (equivalent to linker length of 70 to $90 \mathrm{bp}$ ) had a chromatin fibre diameter of $\sim 45 \mathrm{~nm}$ (Robinson et al., 2007). These measurements were used to model the trajectory of the linker DNA within the chromatin fibre (Wong et al., 2007). These authors show that the most energetically favourable chromatin fibre conformation for each class of linker length is likely to result in a different chromatin fibre structure.

A relationship between intron length and nucleosome repeat length was suggested following examination of human and mouse exon and intron lengths (Beckmann and Trifonov, 1991). Periodicities between 200 and 210 bp were detected within the exon/intron and intron/exon length distributions for both human and mouse datasets. The periodicity detected was found to be sustained over long distances $2000 \mathrm{bp}$. This led to the suggestion that introns may be contributing to the overall chromatin higher-order structure, by maintaining nucleosome positioning signals within genic DNA. The $[\mathrm{AA} / \mathrm{TT}]$ nucleosome positioning signal was used in a computational study to investigate nucleosome positioning around exon/intron and intron/exon boundary sequences (Dennisov, et al., 1997). This study used a set of $\sim 4000$ sequences mainly from the human and mouse genomes, and revealed that the splice junction is positioned within the mid 15 bases of the nucleosome (dyad axis). It was suggested that the placement of nucleosomes over the splice junction may provide protection of the splice junction against mutation. A computational study used experimentally determined and predicted splice-site sequence data to investigate dinucleotide phasing around splice sites for a number of species (Kogan and Trifonov, 2005). This study was able to position both the exon/intron and intron/exon boundaries to 1-2 bp from the nucleosome mid-point (dyad axis), providing further evidence of protective nucleosome positioning at gene splice-sites.

In Arabidopsis, the 18S, 5.8S and 25S rRNA genes are organised into two clusters (nucleolar organiser regions (NORs)) of $700 \pm 130$ tandemly repeated copies on chromosomes 2 and 4, with a single repeat present on chromosome 3 (European Union Chromosome 3 Arabidopsis Genome Sequencing Consortium, 2000). The rRNA genes are separated by two internal transcribed spacer (ITS) sequences and repeats are separated by an intergenic spacer (IGR) (Figure 4.1). The coding sequences are transcribed by RNA polymerase I (Pol I). The rRNA transcripts 
Nucleosome spacing and linker length variation constitute 50-80\% of transcribed RNA in eukaryotic cells, although transcription of only a subset of repeats is needed to fulfil this requirement (Pruess and Pikaard, 2007). The NORs can be visualised by silver staining of metaphase chromosomes, and are referred to as secondary constrictions. These are regions of less condensed chromatin, as previously transcriptionally active rRNA genes remain associated with RNA Pol I transcription factors in order to allow rapid transcription of rRNA in the following cell cycle (McStay, 2006). Silenced rRNA regions are condensed at metaphase and do not form secondary constrictions. Silver staining and FISH of rye NORs on metaphase chromosomes revealed domains of differing chromatin structure within a NOR, with condensed rDNA adjacent to a decondensed secondary constriction, suggesting different regulation of rRNA genes within a NOR (Caperta et al., 2002). Psoralen cross-linking and restriction digestion of murine Friend cell chromatin has demonstrated that two different chromatin states exist in rDNA regions: psoralen accessible (nucleosome depleted) and psoralen inaccessible (organised nucleosomes) (Conconi et al., 1989).

The phenomenon of nucleolar dominance has been used as a model to study the mechanisms of rRNA gene silencing in Arabidopsis. In A. suecica, a naturally occurring allotetraploid of $A$. thaliana and A. arenosa, the NORs originating from the A. thaliana parent are silenced, while the A. arenosa rRNA genes are transcribed (or dominant) (Chen et al., 1998). Treatment of this Arabidopsis hybrid with 5'aza-2deoxycytosine (a methyltransferase inhibitor) or trichostatin A (a histone deacytalase inhibitor) de-represses the A. thaliana rRNA genes. Some A. arenosa rRNA genes are also upregulated following 5'aza-2-deoxycytosine treatment, suggesting that a subset of these genes is also silenced in the Arabidopsis hybrid, and it is likely that the same mechanism controls gene silencing and rRNA gene dosage. Further work revealed that the promoters of the A. thaliana rRNA genes in A. suecica are heavily methylated and the genes are associated with $\mathrm{H} 3 \mathrm{~K} 9 \mathrm{me} 2$, a known heterochromatic mark. The transcribed A. arenosa rRNA gene promoters are hypomethylated and associated with the euchromatic mark $\mathrm{H} 3 \mathrm{~K} 4 \mathrm{me} 3$, whilst the silenced subset of $A$. Arenosa rRNA genes are associated with $\mathrm{H} 3 \mathrm{~K} 9 \mathrm{me} 2$ and show hypermethylation at the promoter (Lawrence et al., 2004). 


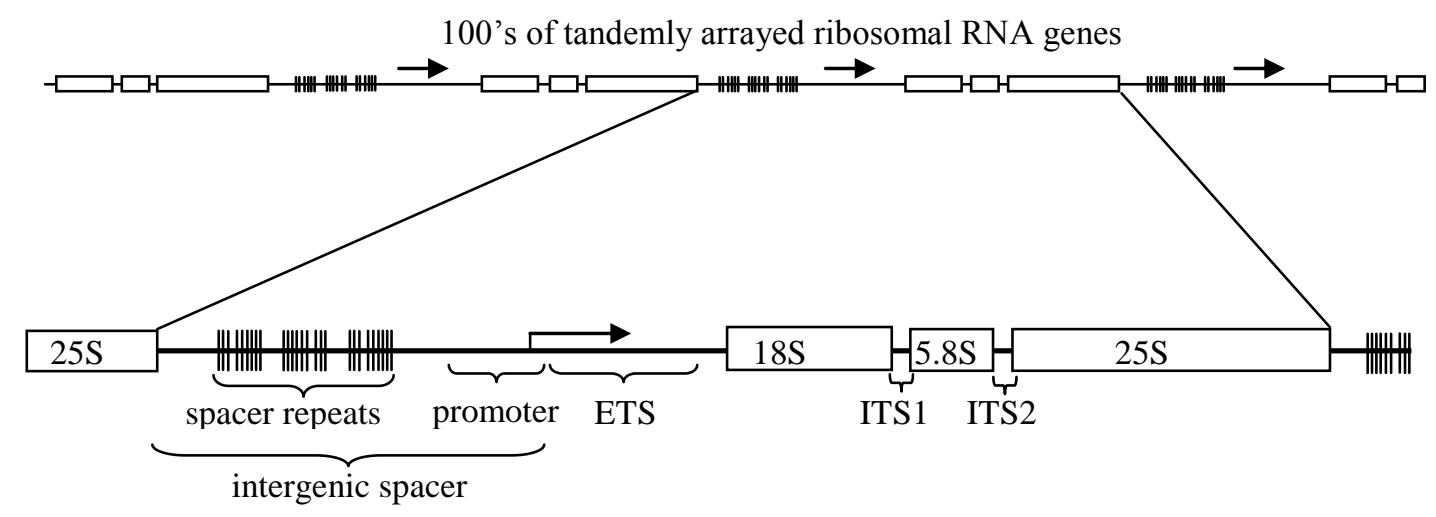

Figure 4.1 Organisation of the 18S, 5.8S and 25S rRNA genes, the internal transcribed spacers and intergenic spacer. Image reproduced from Pruess and Pikaard (2007).

Considering the important contribution of linker length variation to chromatin higher-order structure, the following hypotheses were constructed and tested:

1. The distribution of linker length in Arabidopsis is comparable to that determined for other taxa.

2. Periodicity exists within the Arabidopsis linker length distribution similar to that identified in other taxa.

3. Features of the observed distribution of Arabidopsis linker length show similarities to the distribution of Arabidopsis intron length, which may indicate a relationship between introns in the DNA and chromatin structure.

4. A distinct linker length distribution exists within the nucleolar organiser regions of the Arabidopsis genome, which reflects region-specific higherorder chromatin structure. 


\subsection{Methods}

\subsubsection{Calculation of linker length}

Linker DNA lengths were calculated by subtraction of 294 bp (2 x nucleosome length (147 bp)) from the dinucleosome lengths of fragments for each of the data sets Ath_01_wt, Ath_03_wt and Ath_04_MET1.

Linker lengths were estimated from mononucleosome positions for each of datasets Ath_02_wt and Ath_04_MET1 by calculating the distance between the end of a nucleosome position and the start of the adjacent nucleosome position from the start position of nucleosome, using the formula $\left[n+1_{\text {start }}-n_{\text {end }}\right]$ as shown in Figure 4.2A.

Where $n_{\text {end }}-n+1_{\text {start }}<0$, the lengths were omitted as they represent overlapping nucleosome positions (nucleosome in alternative positions in different cells) rather than linker lengths (nucleosome spacing) as shown in Figure 4.2B.

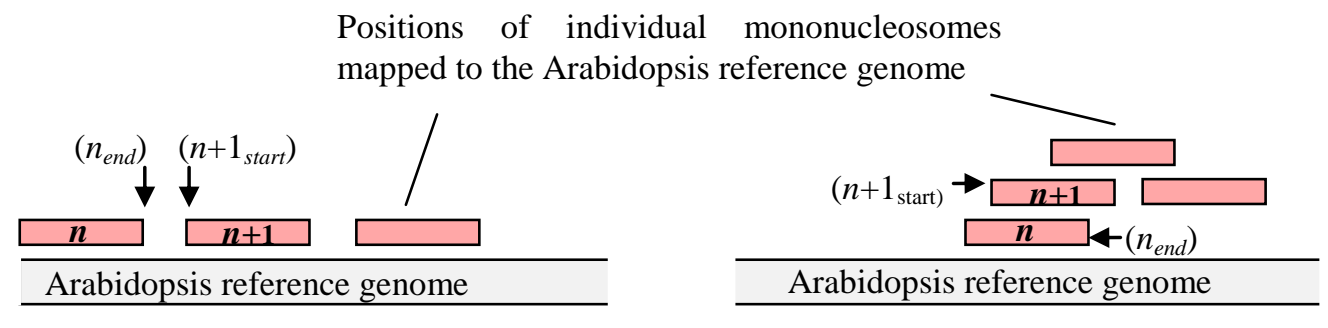
A. Adjacent positions
B. Overlapping positions

Figure 4.2 Schematic showing the method for estimation of linker length from mononucleosome DNA datasets, A: shows positions of adjacent nucleosome and B: shows overlapping nucleosomes, which are likely to represent alternative positions for the same nucleosomes from different cells.

\subsubsection{Calculation of length periodicities}

Fourier analyses and partial autocorrelations were performed on frequencies calculated in section 4.2.1 in order to determine any periodicities in linker length distribution. The procedures are described in Chapter 2, Section 2.2.iv. 
4.2.3 Determination of Arabidopsis exon and intron length and periodicity

Intron sequences and annotations were downloaded from the TAIR (v8) blast dataset ftp site (url: ftp://ftp.arabidopsis.org/home/tair/Sequences/blast_datasets). Intron lengths were measured directly from the sequence lengths. Exon lengths were inferred by subtracting the start position of intron 2 from the end position of intron 1 , for introns $i,\left(i+1_{\text {start }}-i_{\text {end }}\right)$ as shown in Figure 4.3. The distances from the start of an intron to the start of the next intron $\left(i+1_{\text {start }}-i_{\text {start }}\right)$, and from the start on an intron to the end of the next intron were also calculated.

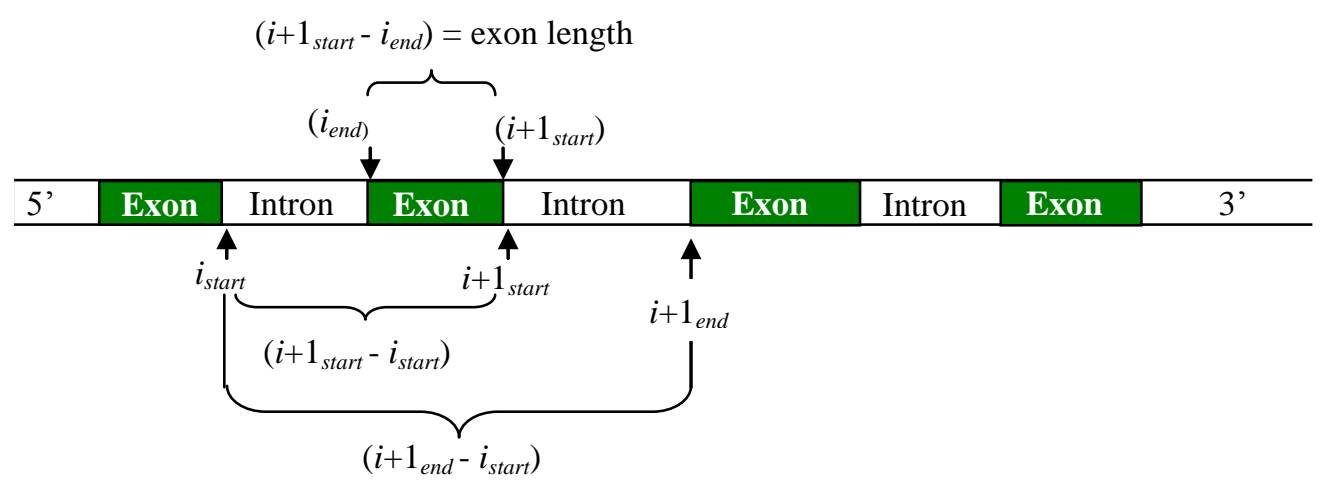

Figure 4.3 Schematic showing the method for calculating exon and intron lengths from intron position data and for calculating intron/exon combination datasets.

Any periodicities in the distributions of exon and intron lengths were determined by Fourier analysis as previously described.

4.2.4.i Determination of linker length within an Arabidopsis nucleolar organiser region

Dinucleosome DNA fragments from dataset Ath_03_wt which mapped within 1000 bp* in the 5' region of a miscellaneous RNA gene and within $1000 \mathrm{bp}$ in the 3' region of the 18S rRNA genes, repeated on chromosomes 2 and 3, were extracted. The data sub-sets and regions represented from each chromosome are shown in Table 4.1. The two regions (one from each chromosome) contain the $5 \mathrm{~S}$ and $18 \mathrm{~S}$ rRNA genes, of which $730 \pm 100$ copies are thought to be present on $\mathrm{Chr} 2$, and correspond to the nucleolar organiser region (NOR) on that chromosome. The repeats are not accounted for in the annotated reference genome.

*Only 871 bp upstream of the AT2G01008 gene as this is the edge of the sequence data available (TAIR v8). 
Nucleosome spacing and linker length variation

The region on Chr 3 is thought to contain a single copy of the repeat. Only fragments which mapped to unique sequences on either $\mathrm{Chr} 2$ or Chr 3 were used for the analyses.

Table 4.1 The two subsets extracted from the Ath_03_wt dataset which represent the annotated rRNA regions on Chromosomes 2 and 3. The gene models, descriptions and positions relative to the annotated reference genome are shown.

\begin{tabular}{c|lllrrr}
\hline Dataset & $\begin{array}{c}\text { Dataset region } \\
\text { (bp) }\end{array}$ & $\begin{array}{c}\text { Gene model } \\
\text { (TAIR) }\end{array}$ & $\begin{array}{c}\text { Gene } \\
\text { description }\end{array}$ & Chr & $\begin{array}{c}\text { Gene start } \\
\text { position (bp) }\end{array}$ & $\begin{array}{c}\text { Gene stop } \\
\text { position (bp) }\end{array}$ \\
\hline Ath_03_rna2 & 1000 to & AT2G01008 & other RNA & 2 & 1,871 & 2,111 \\
& 6,782 & AT2G01010 & 18S rRNA & 2 & 3,706 & 5,513 \\
& & AT2G01020 & 5S rRNA & 2 & 5,782 & 5,945 \\
& & & & & & \\
Ath_03_rna3 & $14,205,903$ to & AT3G41761 & other RNA & 3 & $14,206,903$ & $14,207,064$ \\
& $14,211,902$ & AT3G41768 & 18S rRNA & 3 & $14,208,663$ & $14,210,470$ \\
& & AT3G41979 & 5S rRNA & 3 & $14,210,739$ & $14,210,902$ \\
\hline
\end{tabular}

Linker lengths were calculated for each dataset as described in section 4.2.1, and plotted together with a smoothed curve calculated from an $8 \mathrm{bp}$ sliding window. The latter removed small-scale periodicity and revealed overall trends in the distribution.

\subsection{4.ii Kernel Density Estimation}

In order to inspect the linker length distributions of datasets Ath_03_rna2 and Ath_03_rna3 for multi-modal components within the distribution, Kernel Density Estimation (KDE) was used to smooth the linker length frequency distribution and reveal important longer-range peaks in the distributions. The density estimations were performed in GenStat ${ }^{\circledR}\left(2008,11^{\text {th }}\right.$ Edition, VSN International, UK) with the bandwidth calculated using the method of Sheather \& Jones (1991), and using a set of 1,024 grid points.

\subsection{4.iii Kolmogorov-Smirnov test}

The Kolmogorov-Smirnov test is a non-parametric test used to determine whether two distributions are significantly different, comparing the cumulative distribution function for each. The cumulative distributions of the linker lengths were calculated in GenStat ${ }^{\circledR}\left(2008,11^{\text {th }}\right.$ Edition, VSN International, UK) and the KolmogorovSmirnov $D$-statistic was calculated as the largest difference between the cumulative distributions at any value of $x$, where $x$ represents increasing linker length. 
The critical D-value was calculated as:

$D \alpha=c(\alpha) \sqrt{\frac{n_{1}+n_{2}}{n_{1} n_{2}}}$

where $c(\alpha)=1.36$ when $\alpha=0.05$, and where $n_{1}$ and $n_{2}$ are the numbers of observations in the two sets of linker lengths (distributions) being compared.

The critical $D$-value is compared to the calculated Kolmogorov-Smirnov $D$-statistic. A calculated $D$-value larger than the critical value indicates a statistically significant difference between distributions at the $\alpha$ (or $\mathrm{p})=0.05$ level of significance. 


\section{Results}

\section{$\underline{\text { 4.3.1 Linker length distribution }}$}

To test the hypothesis that linker DNA in Arabidopsis is variable in length and has a non-random in distribution, the linker length distribution was investigated using the datasets Ath_01_wt,Ath_02_wt,Ath_03_wt and Ath_04_MET1.

\subsection{1.i Calculation of linker length from dinucleosome DNA fragments}

For dinucleosome sequences (DNA fragments with a length $>294$ bp) from datasets Ath_01_wt, Ath_03_wt and Ath_04_MET1, linker lengths were calculated by subtracting twice the nucleosome length (147 bp, Richmond and Davey, 1993) from the dinucleosome DNA fragment length (see Figure 4.4). However, this does not take into consideration any minimum linker length, steric hindrance, nor the presence of histone H1. Fragment lengths less than 294 were omitted from the analysis as this is the minimum possible dinucleosome length ( 2 x $147 \mathrm{bp}$ ). Using these constraints, it is possible that dinucleosome fragments length over 442 bp (linker length $=147$ bp) may actually originate from a tri-nucleosome fragment with no linker DNA. For this reason, fragments over $442 \mathrm{bp}$ in length were also omitted from the analysis. The distributions of linker length for datasets Ath_01_wt, Ath_03_wt and Ath_04_MET1 are shown in Figure 4.5

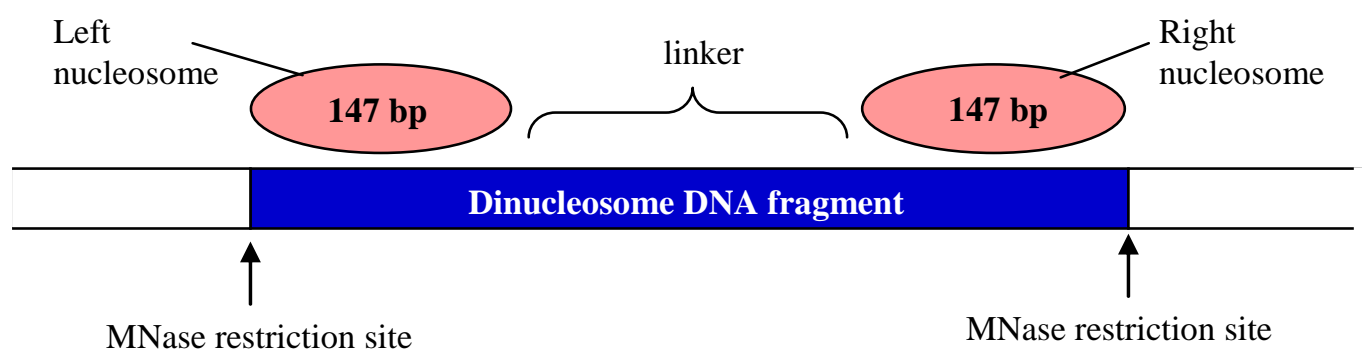

Figure 4.4 Schematic showing the calculation of linker length by subtracting 2x 147 bp (two nucleosomes) from the length of a dinucleosome DNA fragment. 

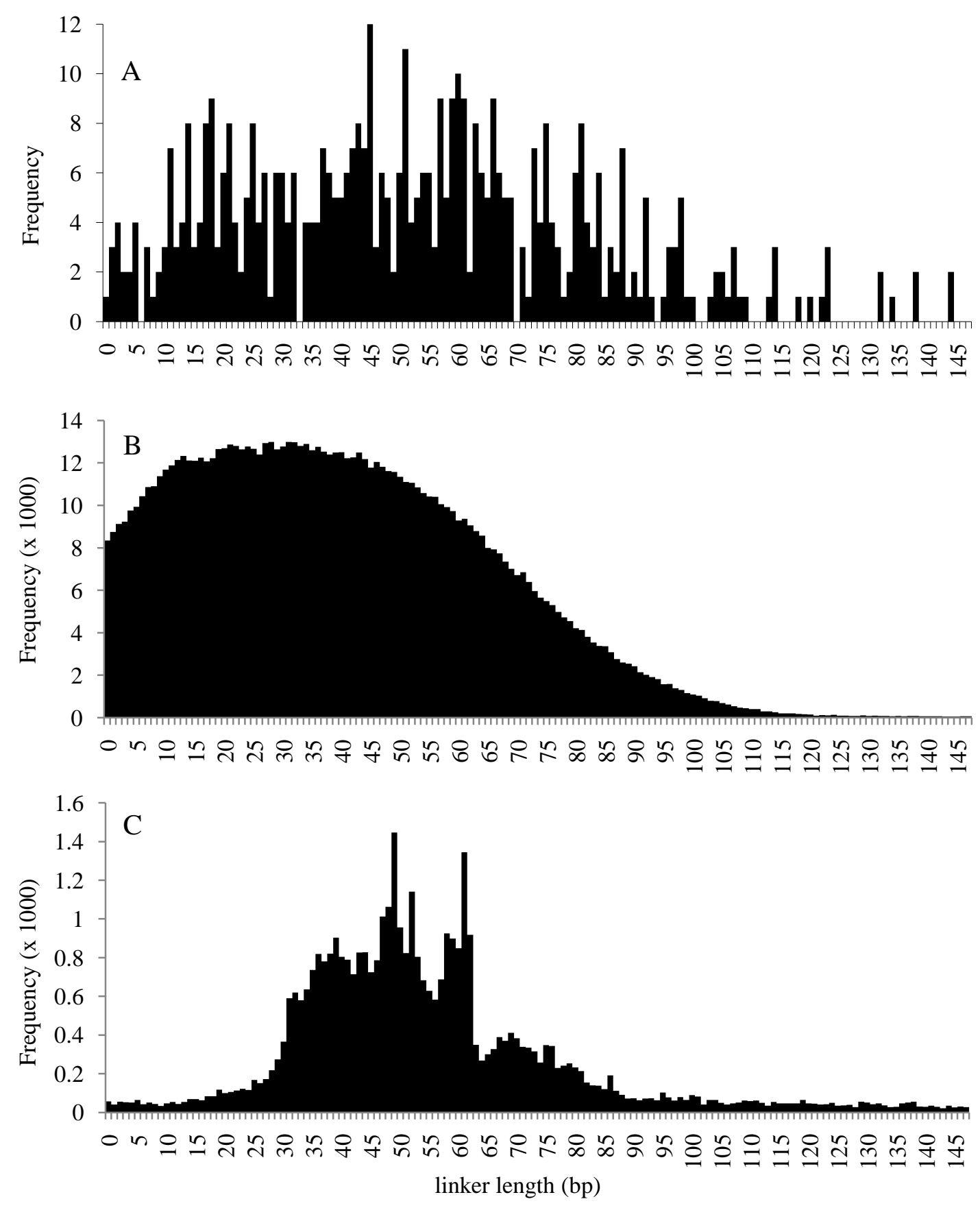

Figure 4.5 Distributions of calculated linker lengths from dinucleosome-length sequences from the datasets A: Ath_01_wt B: Ath_03_wt and Ath_04_MET1.

The Ath_01_wt $(\mathrm{n}=485)$ and Ath_04_MET1 $(n=39,608)$ datasets show non-random distributions of linker length with multiple peaks within the distributions. The peaks are likely to represent the distributions around preferred linker lengths for Arabidopsis dinucleosomes. The Ath_03_wt dataset $(n=903,305)$ does not appear to show such peaks within the distribution, which could be due to the larger sample size 
Nucleosome spacing and linker length variation (or under-sampling of the other datasets). The distributions of Ath_01_wt and Ath_O4_MET1 suggest that there is a multi-modal distribution for linker length.

The mean linker length $(\bar{y})$ and standard deviation were calculated for each dataset and are shown in Table 4.2. This shows that there appears to be more similarity between the mean linker lengths of the Ath_O1_wt and Ath_04_MET1 datasets, with the medians being identical. The standard deviations are large for each data set, and indicate a large range of linker lengths in Arabidopsis.

Table 4.2 Linker lengths calculated from Arabidopsis dinucleosomes from the datasets Ath_01_wt, Ath_03_wt and Ath_04_MET1

\begin{tabular}{l|rrrr}
\hline Data set & \multicolumn{2}{|c}{$\begin{array}{l}\text { Mean }(\bar{y}) \\
(\mathrm{bp})\end{array}$} & $\begin{array}{l}\text { Median } \\
(\mathrm{bp})\end{array}$ & $\begin{array}{l}\text { Standard } \\
\text { deviation }\end{array}$ \\
\hline Ath_01_wt & 485 & 52.39 & 51 & 29.46 \\
Ath_03_wt & 903,305 & 40.01 & 38 & 24.89 \\
Ath_04_MET1 & 39,608 & 54.67 & 51 & 23.10 \\
\hline
\end{tabular}

\subsection{1.ii Derived linker lengths from mononucleosome positions}

In order to estimate the linker length variation in the mononucleosome datasets, the distances between the positions of mononucleosomes were calculated for the Ath_O2_wt and Ath_04_MET1 datasets. Linker lengths were calculated as the distance between individual mononucleosome DNA fragments mapped onto the Arabidopsis genome, as shown in the schematic (Figure 4.6). Distances less than $147 \mathrm{bp}$ (or linker length < 0) were omitted from the analysis as these lengths represent alternative nucleosome positions. In addition, distances larger than $294 \mathrm{bp}$ (linker lengths > 147) were omitted from the analysis as beyond this length, there is no certainty that the distance represents dinucleosome linkers (see section 4.3.1.i.).

The distributions of the derived linker lengths for datasets Ath_O2_wt $(n=1,545)$ and Ath_04_METI $(n=154,084)$ are shown in Figure 4.7 . 
Nucleosome spacing and linker length variation

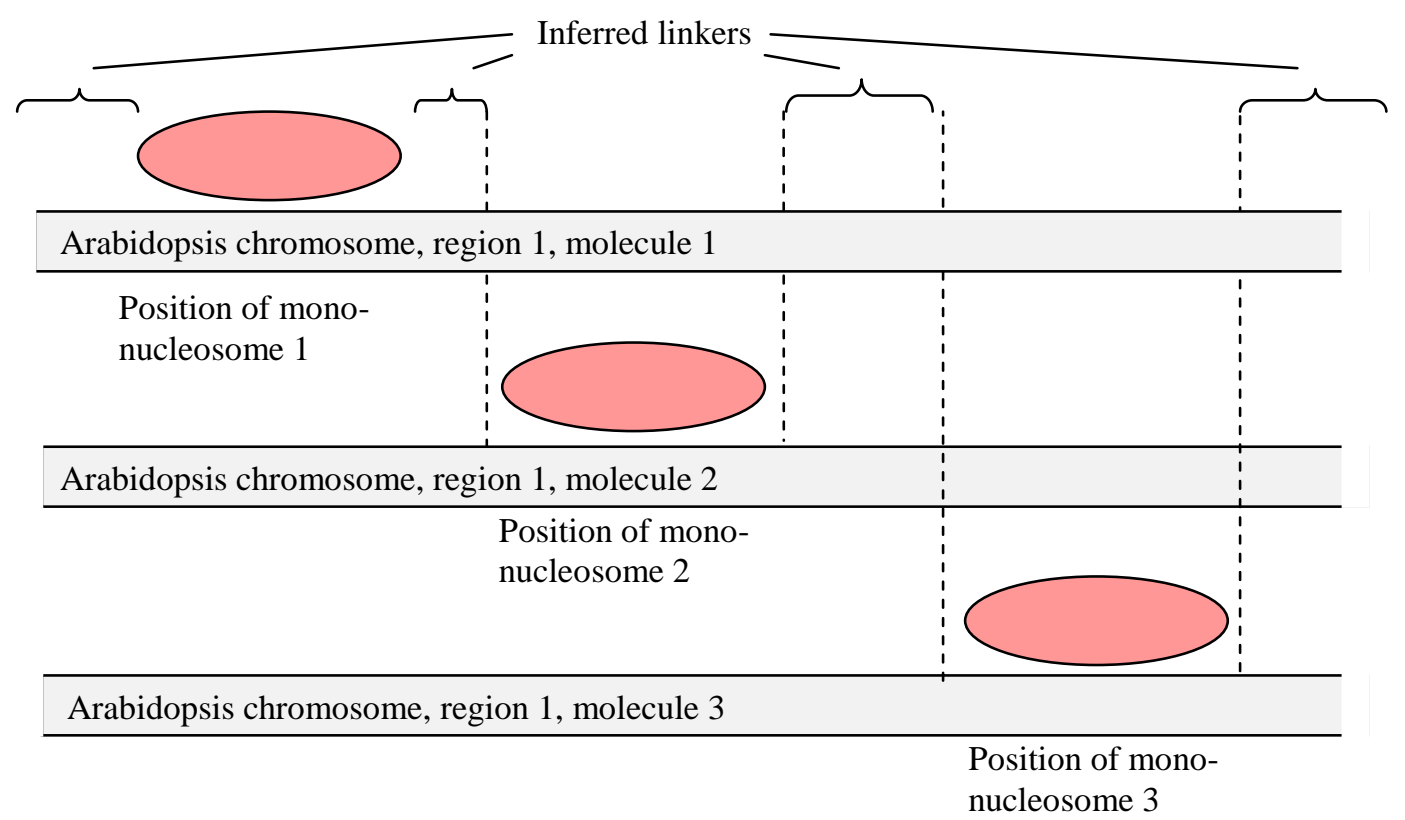

Figure 4.6 Schematic showing the method for inferring linker lengths from a set of mononucleosome DNA fragments. The diagram demonstrates different nucleosome positions in different molecules over the same region of an Arabidopsis chromosome.
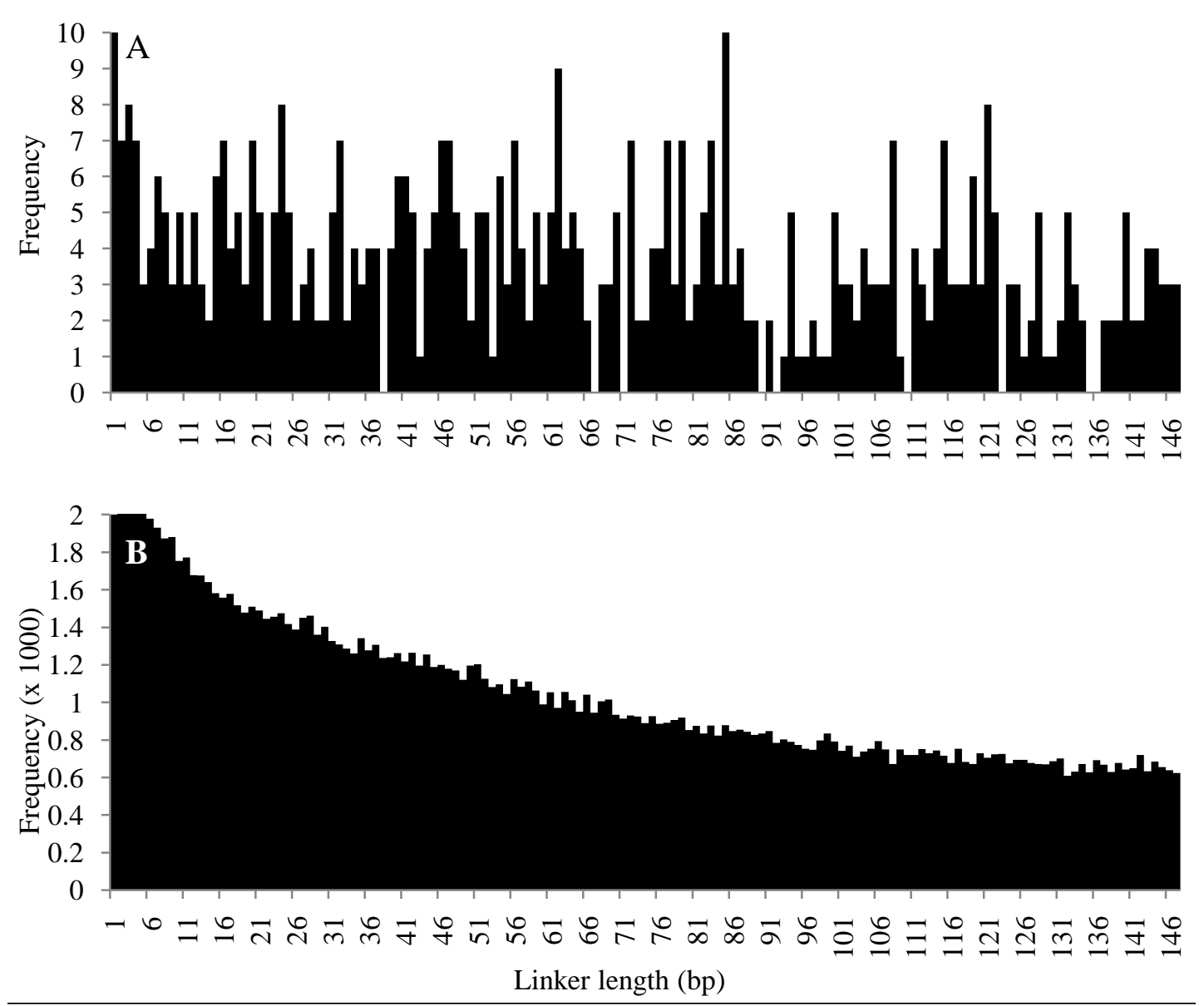

Figure 4.7 Distribution of the inferred linker lengths from datasets, A: Ath_02_wt and B: Ath_04_MET1. 
Nucleosome spacing and linker length variation The linker lengths derived from the Ath_O2_wt data show a distribution similar to that of the Ath_01_wt linker lengths with peaks throughout the distribution, possibly representing preferred length for linker DNA in Arabidopsis. The distribution of the Ath_04_MET1 derived linker lengths is more continuous, with less well defined maxima within the distribution. It may be that the smaller datasets are undersampled, showing only a subset of the dinucleosome lengths present in the genome.

The mean linker length $(\bar{y})$ and standard deviation were calculated for each dataset and are shown in Table 4.3. The mean and median values for the derived linker lengths are both higher than those from the linker lengths calculated from dinucleosome DNA fragments.

Table 4.3 Mean, median and standard deviation of linker lengths derived from mononucleosome positions using the datasets Ath_02_wt and Ath_04_MET1.

\begin{tabular}{l|lrrr}
\hline Dataset & $\begin{array}{l}\text { Number of } \\
\text { values }\end{array}$ & $\begin{array}{l}\text { Mean }(\bar{y}) \\
(\mathrm{bp})\end{array}$ & $\begin{array}{l}\text { Median (bp) } \\
\begin{array}{l}\text { Standard } \\
\text { deviation } \\
\text { (bp) }\end{array}\end{array}$ \\
\hline Ath_02_wt & 549 & 64.58 & 61 & 42.64 \\
Ath_04_MET1 & 155,566 & 58.95 & 52 & 42.43 \\
\hline
\end{tabular}


Nucleosome spacing and linker length variation 4.3.2 Periodicity in linker length distribution

Previous studies have presented evidence suggesting that a periodicity of approximately 10 bp exists in linker length distributions (Kato et al., 2003, Widom et al., 1992). The periodicity is thought to be a consequence of nucleosome positioning influenced by the DNA helical twist. In order to test the hypothesis that Arabidopsis chromatin exhibits preferred nucleosome linker lengths, periodicity was investigated using Fourier analysis and partial autocorrelation. Linker lengths calculated in the previous sections 4.3.1.i. and 4.3.1.ii. for datasets Ath_01_wt, Ath_02_wt, Ath_03_wt and Ath_04_MET1 were used for the analyses.

The results of Fourier analyses of the distribution of linker lengths in Arabidopsis are shown in Table 4.4, and suggest a periodicity of $7.68 \mathrm{bp}$ for the Ath_01_wt, $7.45 \mathrm{bp}$ for Ath_02_wt and $7.34 \mathrm{bp}$ for Ath_03_wt dinucleosome datasets. A periodicity between $9.2 \mathrm{bp}$ and $9.8 \mathrm{bp}$ was calculated for the linker length from the dinucleosome fragments of Ath_04_MET1 and a periodicity of 12.25 bp was calculated for the distribution of distances between nucleosome positions from the Ath_04_MET1 dataset. The partial autocorrelation function (PACF) is described as the correlation between observations $n_{l}$ and $n_{l+k}$, having accounted for all other correlations from $n_{l}$ to $n_{l+k-1}$, and gives an indication of periodicity in the data. PACFs were calculated from the linker length distributions for Arabidopsis datasets Ath_01_wt, Ath_02_wt, Ath_03_wt and Ath_04_MET1 and are shown in Table 4.4. In general, the PACF tends to support the periodicity calculated by Fourier analysis.

The calculated linker length periodicity for Arabidopsis is less than the $\sim 10 \mathrm{bp}$ previously reported for other taxa (Kato et al., 2003, Widom et al., 1992). In order to provide a direct comparison, the Fourier and partial autocorrelation analyses were performed using dinucleosome DNA fragments from the publicly available datasets: Kato_03_hum, Kato_05_hum, and Trav_chick_dinuc. In addition, distances between mononucleosome positions from the Johns_Celegans dataset were also used (Table 4.4). The periodicities in the linker length distributions derived from these datasets have not previously been calculated. Descriptions of these datasets can be found in the general materials and methods (Chapter 2, section 2.2.vii). The periodicities calculated by Fourier analysis and PACF are shown in Table 4.4. A periodicity of 8 
Nucleosome spacing and linker length variation bp was detected within the distribution of the Kato_03_hum dataset while a periodicity of 6.34 bp was calculated for the Kato_05_hum dataset. The discrepancy between these two datasets could be due to the small sampling size of the Kato_05_hum set $(n=100)$ compared to the Kato_03_hum dataset $(n=1002)$. The partial autocorrelation suggests a periodicity of 8-9 bp for the linker length distribution in both datasets, which is the closest to the $\sim 7$ bp detected here for Arabidopsis. The Trav_chick_dinuc dataset was the only one which exhibited the reported 10 bp periodicity within the linker length distribution, which is also seen in the PACF. The Johns_Celegans dataset exhibits a periodicity of $\sim 5$ bp within the linker length distribution, although there was also some evidence for a periodicity of 9.75 bp for this dataset. A periodicity of 4 bp was detected in the Johns_Celegans linker length distribution using PACF, which is closer to the $\sim 5$ bp Fourier analysis result, suggesting a periodicity of $4-5 \mathrm{bp}$ for this dataset. Overall, these results suggest that linker length preferences may be taxa-specific.

Table 4.4 Statistically significant $(p<0.05)$ periodicities calculated by Fourier analyses and PACF for linker length distributions derived from Arabidopsis datasets Ath_01_wt, Ath_02_wt, Ath_03_wt and Ath_04_MET1. In addition, the periodicities of Kato_03_hum, Kato_05_hum, Trav_chick_dinuc and Johns_Celegans are shown.

\begin{tabular}{l|lrrr}
\hline \multicolumn{1}{c|}{ Dataset } & Linker length derived from: & $\begin{array}{c}\text { Periodicity } \\
\text { Fourier } \\
\text { (bp) }\end{array}$ & $\begin{array}{c}\text { Confidence limit } \\
\text { (Fourier) (bp) }\end{array}$ & $\begin{array}{c}\text { Periodicity } \\
\text { (PACF) (bp) }\end{array}$ \\
\hline Ath_01_wt & dinucleosome fragment & 7.68 & $7.30-8.30$ & 7 \\
Ath_03_wt & dinucleosome fragment & 7.34 & $6.68-7.73$ & 6 \\
Ath_04_MET1 & dinucleosome fragment & $9.2-9.8$ & $8.7-10.50$ & 10 \\
Ath_02_wt & mononucleosome position & 7.45 & $7.10-7.84$ & 8 \\
Ath_04_MET1 & mononucleosome position & 12.25 & $11.31-13.37$ & 14 \\
Kato_03_hum & dinucleosome fragment & $* 8.00$ & $7.62-8.89$ & $8-9$ \\
Kato_05_hum & dinucleosome fragment & 6.34 & $6.00-6.53$ & 8 \\
Trav_chick_dinuc & dinucleosome fragment & 10.66 & $10.00-11.43$ & $9-10$ \\
Johns_Celegans & mononucleosome position & $* * 5.20$ & $5.12-5.29$ & 4 \\
\hline * There was also evidence of periodicity of $~ 6$ bp & & & \\
** There was also evidence of periodicity of 9.75 bp & & &
\end{tabular}


Nucleosome spacing and linker length variation

\subsubsection{Relationship between linker length and intron length}

Previous studies using the nucleosome positioning signal [AA/TT] dinucleotide have shown that nucleosomes are likely to be positioned at 5'intron/exon3' boundaries (Denisov et al., 1997). Further modelling based on sequence periodicities around intron/exon boundaries suggested that the nucleosome dyad axis is likely to be positioned one or two bases upstream of the intron/exon boundary (Kogan and Trifonov, 2005). This would place half of the nucleosome in the exon and half in the intron. If nucleosomes are positioned at both the 5'intron/exon3' and 5'exon/intron3' boundaries, it may be expected that average intron length is related to linker length. In order to test the hypothesis that such a relationship exists in Arabidopsis, the lengths of exons, introns and the distance from the start of an intron to the end of the following exon (5'intron-exon 3 ') and the distance from the start of an intron to the end of the following intron (5 'intron-exon-intron3') were calculated as shown in the schematic (Figure 4.8).

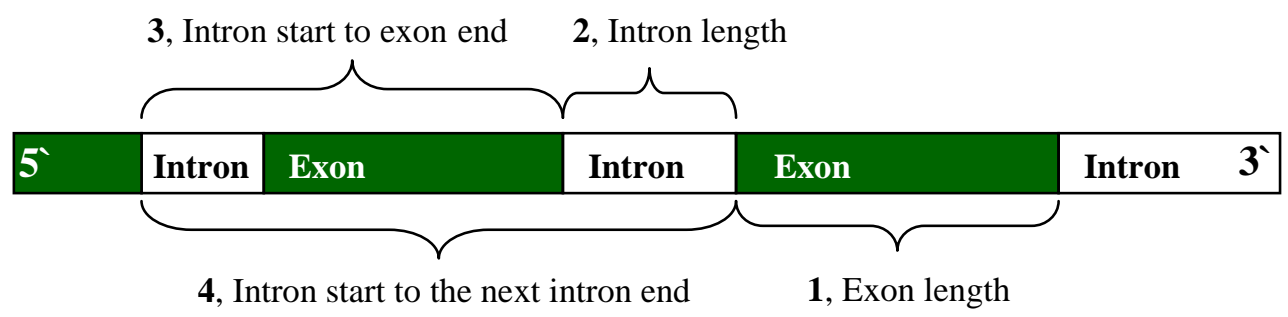

Figure 4.8 Schematic showing how lengths of exons (1), introns (2), 5'intron-exon3' (3) and 5'intron-exon-intron3' (4) were determined.

The length distributions were calculated and are shown in Figure 4.9. The mean $(\bar{y})$, median and standard deviations of the lengths for the exon (' 1 ' in Figure 4.8), intron ('2' in Figure 4.8), 5'intron-exon3' ('3' in Figure 4.8) and 5'intron-exon-intron3' ('4' in Figure 4.8) datasets were calculated. The exon DNA fragments have a maximum in the distribution (modal group) at $73 \mathrm{bp}$, coinciding with a halfnucleosome distance (73.5 bp). The maxima for the intron, 5'intron-exon3' and 5 'intron-exon-intron3' DNA fragments were 85, 182 and 275 bp respectively. 
Nucleosome spacing and linker length variation
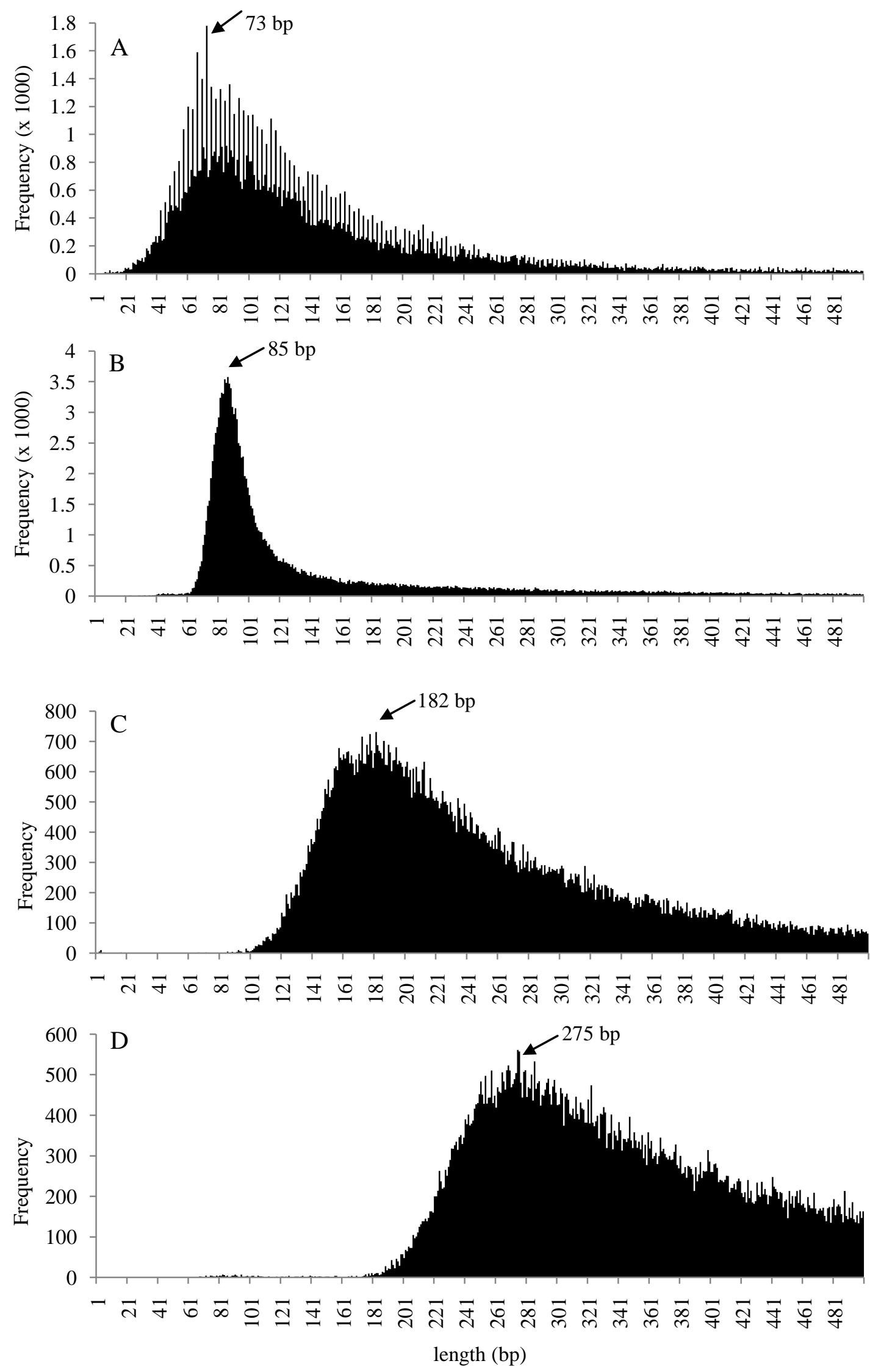

Figure 4.9 Distributions of the length of Arabidopsis genomic components, A: exons, B: introns, $C$ : the distances from intron start to exon end, and $D$ : the distance from intron start to the next intron end. 
Nucleosome spacing and linker length variation

To further investigate the nucleosome positioning at 5'intron/exon3' boundaries, periodicities within the distributions of the exon, intron, 5'intron-exon3' and 5'intron-exon-intron3' datasets were determined using Fourier analysis. The calculated periodicities are shown in Table 4.5. A periodicity of $3 \mathrm{bp}$ was detected within the exon length distribution. This is most likely the signal associated with the 3 bp codon length reported previously (Choon and Yan, 2008). A periodicity of $\sim 7$ bp was detected in the distribution of Arabidopsis intron length which is close to the periodicity detected within the distribution of linker lengths. The periodicities of $8.3 \mathrm{bp}$ detected within the distributions of the 5 'intron-exon-3'and the 5 'intron-exonintron3' datasets are different to the $\sim 7$ bp detected in the distribution of linker lengths.

Table 4.5 The mean, standard deviation and median lengths for Arabidopsis exons, introns, intron start to exon end and from intron start to the next intron end. In addition, periodicities in the distribution, obtained by Fourier analysis, for each feature are shown.

\begin{tabular}{l|rrrrrrr}
\hline \multicolumn{1}{c|}{ Dataset } & $\begin{array}{c}(\bar{y}) \\
(\mathrm{bp})\end{array}$ & $\begin{array}{c}\text { Median } \\
(\mathrm{bp})\end{array}$ & $\begin{array}{c}\text { Standard } \\
\text { Deviation } \\
(\mathrm{bp})\end{array}$ & Kurtosis & Skewness & $\begin{array}{c}\text { Periodicity } \\
\text { (Fourier) } \\
(\mathrm{bp})\end{array}$ & $\begin{array}{c}\text { Confidence } \\
\text { limit } \\
(\mathrm{bp})\end{array}$ \\
\hline exon & 170 & 116 & 202 & 98.30 & 7.07 & 3.00 & $2.99-3.01$ \\
intron & 165 & 99 & 179 & 174.07 & 7.87 & 7.19 & $7.14-7.24$ \\
5' 'intron-exon3' & 322 & 242 & 265 & 74.73 & 5.70 & 8.33 & $8.26-8.40$ \\
5'intron-exon- & 474 & 375 & 321 & 52.79 & 4.64 & 8.36 & $8.26-8.40$ \\
intron3' & & & & & & & \\
\hline
\end{tabular}

The above analyses were repeated for the first introns relative to the 5 , end of transcriptional units, as a subset, since first introns have been shown to possess distinct properties from subsequent introns (Jeong, et al., 2006). First introns have been shown to both enhance and inhibit transcription (Rose et al., 2008) and are on average longer in length than subsequent introns (Bradnam and Korf, 2008). The distribution of first intron lengths are shown in Figure 4.10. The mode of the distribution (indicated by an arrow on the histogram) is the same for the first introns as it is for all introns. The mean length is $253 \mathrm{bp}$, with a standard deviation of 268 $\mathrm{bp}$, and the median is $155 \mathrm{bp}$. When tested using Fourier analysis, the distribution of first intron lengths showed a $7.57 \mathrm{bp}$ (CL: $7.51-7.63 \mathrm{bp}$ ) period. This value is slightly higher than the periodicity in the distribution of all introns, and falls within the confidence limits for complete Arabidopsis wild-type datasets. 
Nucleosome spacing and linker length variation

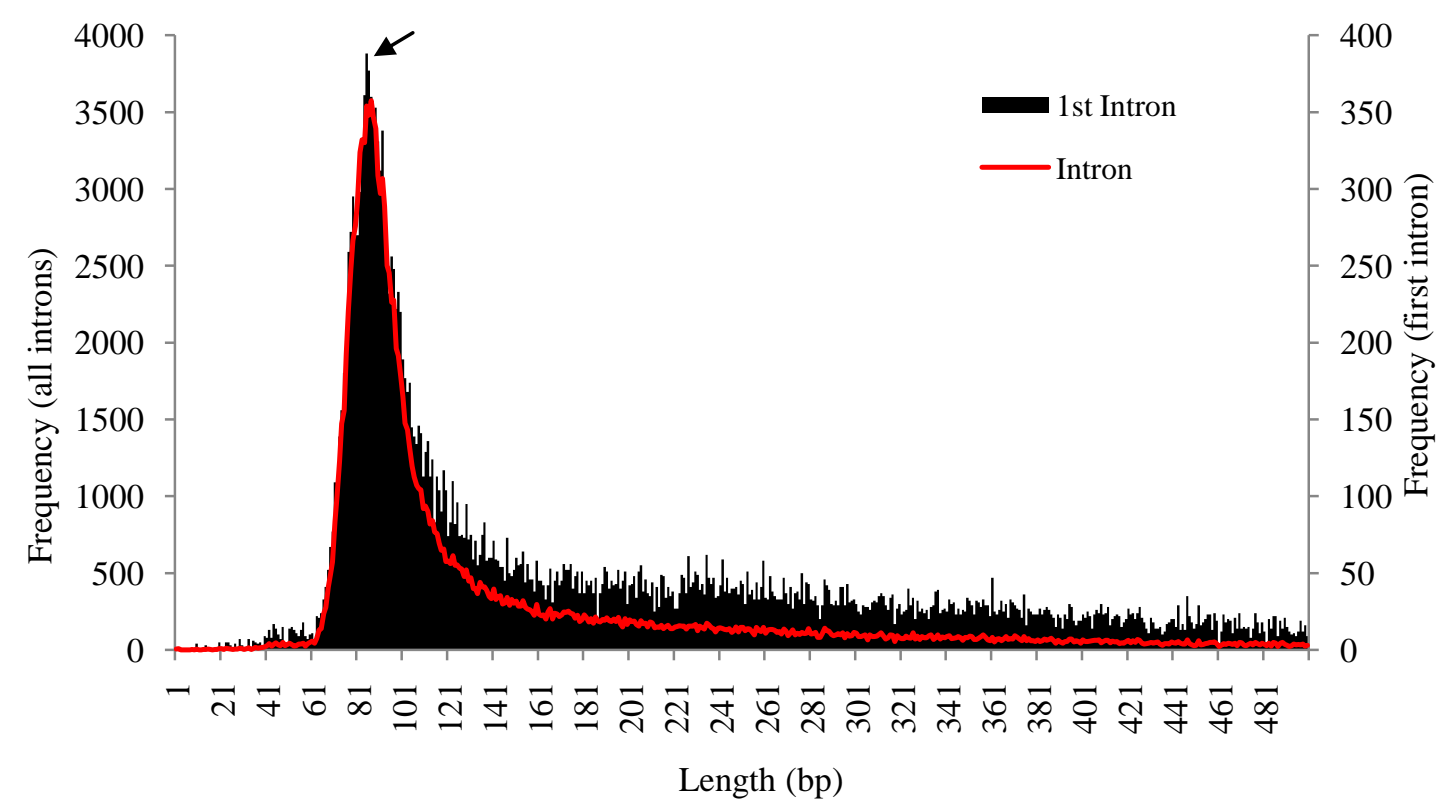

Figure 4.10 Distribution of Arabidopsis first intron lengths, the arrow indicates the mode with a value of 85 bp. The distribution of all introns (from Figure 4.6) is also shown by the red line for comparison. For clarity, the frequencies for the two distributions are shown on different $y$-axes; the frequencies for all introns on the left-hand axis and those for first introns on the righthand axis. 
Nucleosome spacing and linker length variation

\subsubsection{Linker length variation in an Arabidopsis nucleolar organiser region}

In order to test the hypothesis that linker length, and therefore chromatin structure varies between different regions in the Arabidopsis genome, two regions containing similar rRNA gene sequences were compared, these being from 1,000 bp to $6,945 \mathrm{bp}$ on Chromosome 2 and from 14,205,903 bp to 14,211,902 bp on Chromosome 3, as described in the methods (section 4.2.4). Only linker lengths which were calculated from dinucleosome sequences which uniquely map to either $\mathrm{Chr} 2$ or $\mathrm{Chr} 3$ were included in the analyses. The distributions are shown in Figure 4.11. As the Arabidopsis linker length distribution has been shown to have a periodicity of $\sim 7-8$ bp in this study, a smoothed curve over a sliding window of 8 bp was calculated to reveal overall trends in both distributions. This shows a peak in the distribution of linker lengths at $67 \mathrm{bp}$ within the dataset Ath_03_rna2, and two peaks in the distribution of linker lengths, at $30 \mathrm{bp}$ and $42 \mathrm{bp}$, for the Ath_03_rna3 dataset.

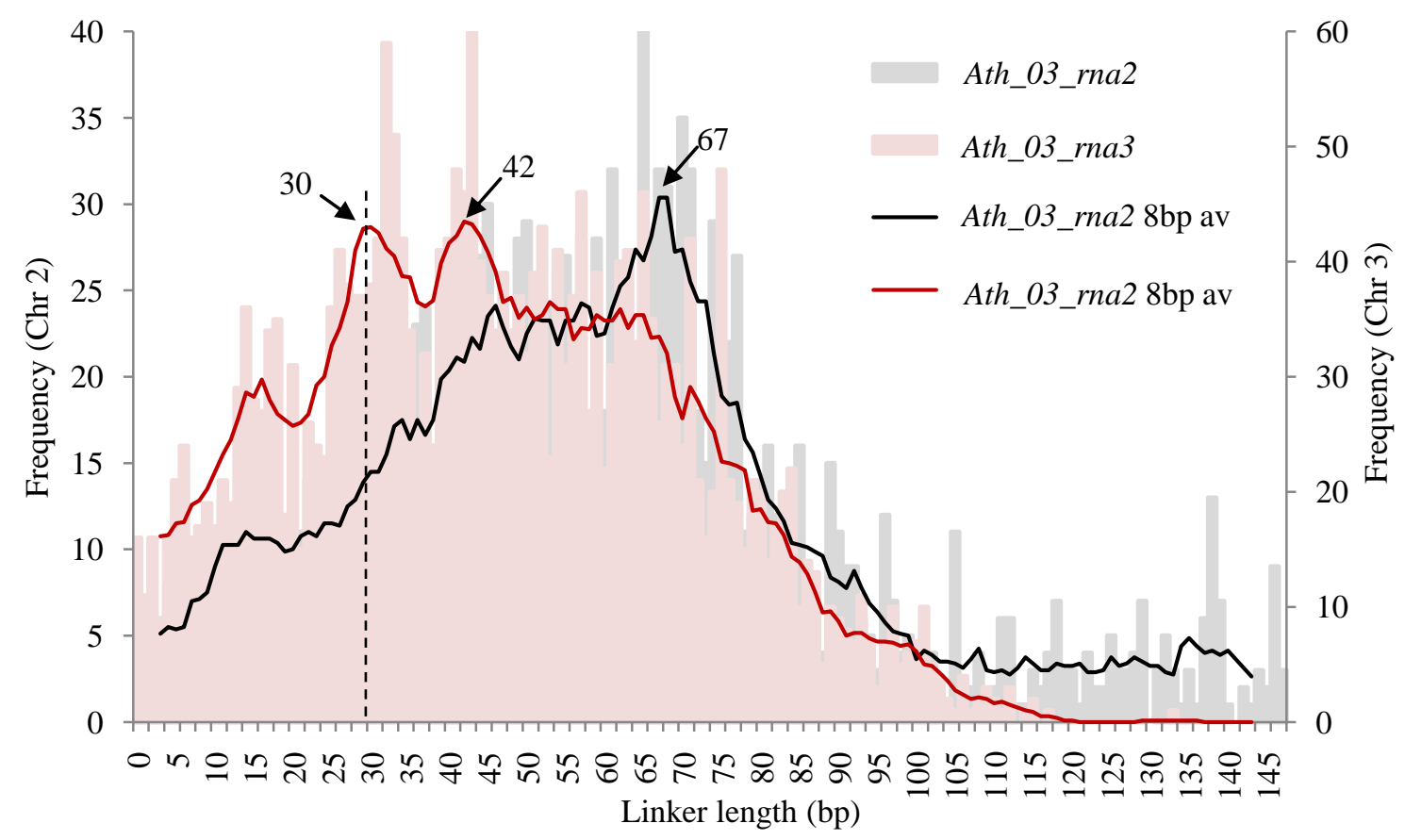

Figure 4.11 Distribution of Linker lengths for datasets Ath_03_rna2 and Ath_03_rna3. An 8 bp moving average is indicated for the Ath_03_rna2 distribution by a black line and for the Ath_03_rna3 distribution by a red line. Peaks at $67 \mathrm{bp}$ in the Ath_03_rna2 distribution and at $30 \mathrm{bp}$ and $42 \mathrm{bp}$ in the Ath_03_rna3 distributions are indicated. For clarity, distributions for datasets Ath_03_rna2 and Ath_03_rna3 are plotted on different $y$-axis scales (Chr2 on the left and Chr3 on the right). 
Nucleosome spacing and linker length variation Basic statistics of the distributions and are shown in Table 4.6. Linker lengths of Ath_03_rna2 have a median of $58.6 \mathrm{bp}$, while the linker lengths from Ath_03_rna3 have a median of $46.5 \mathrm{bp}$. Fourier analysis was used to identify any periodicity in the distribution of linker lengths for each dataset Ath_03_rna2 and Ath_03_rna3 (Table 4.6). The periodicity detected in the distribution of linker lengths from the Ath_03_rna2 is very similar to the periodicity calculated for the whole Ath_03_wt dataset (Table 4.4). However, the periodicity detected in the distribution of linker lengths for the dataset Ath_03_rna3 is higher than that for the whole Ath_03_wt dataset. This suggests that different linker length preferences may exists for different regions in Arabidopsis chromatin.

Table 4.6 Mean, median, standard deviation and periodicity (Fourier analysis) of datasets $A t h \_03 \_r n a 2$ and $A t h \_03 \_r n a 3$.

\begin{tabular}{c|rrrrrr}
\hline Dataset & $n$ & Mean $(\bar{y})$ & Median & $\begin{array}{c}\text { Standard } \\
\text { deviation }\end{array}$ & $\begin{array}{c}\text { Periodicity } \\
\text { (Fourier) }\end{array}$ & $\begin{array}{c}\text { CL for } \\
\text { periodicity }\end{array}$ \\
\hline Ath_03_rna2 & 1719 & 59.63 & 58 & 30.59 & 7.73 & $7.35-8.17$ \\
Ath_03_rna3 & 2752 & 46.49 & 45 & 24.42 & 8.65 & $8.17-9.19$ \\
\hline
\end{tabular}

In order to test for multimodality in the linker length distributions, Kernel Density Estimation was used to smooth the distribution. This allows the identification of important peaks within a distribution. The probability density plots for the datasets Ath_03_rna2 and Ath_03_rna3 are shown in Figure 4.12. The probability density plots show non-normal distributions for both datasets. The Ath_03_rna2 dataset shows a main peak at $67 \mathrm{bp}$ and further peaks at $51 \mathrm{bp}$ and $123 \mathrm{bp}$. The Ath_03_rna3 dataset shows peaks at $43 \mathrm{bp}$ and $51 \mathrm{bp}$, with a further peak observed at $68 \mathrm{bp}$. 


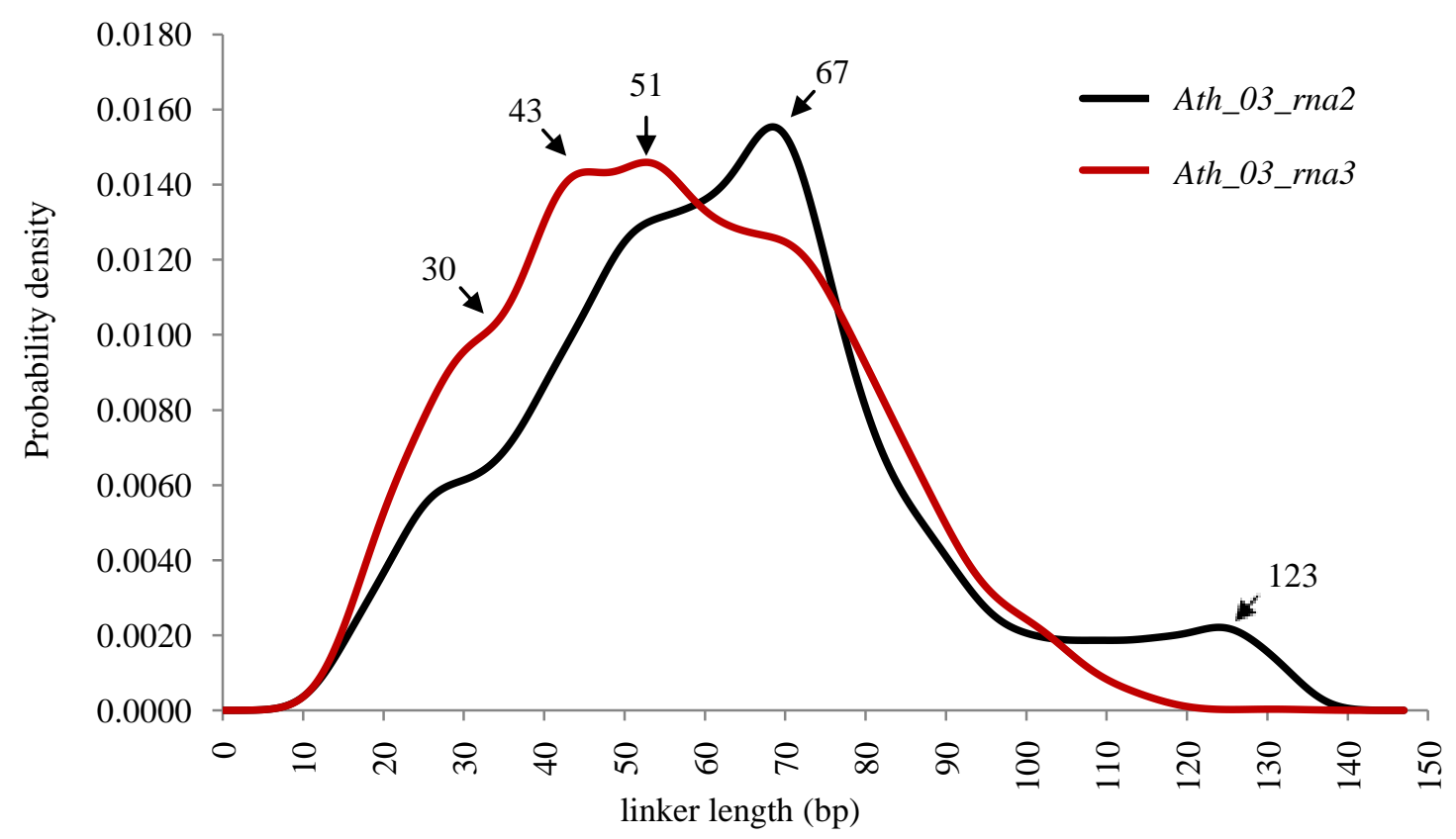

Figure 4.12 Probability density plots for datasets $A t h \_03 \_r n a 2$ and $A t h \_03 \_r n a 3$. The dataset Ath$03 \_r n a 2$ is represented with a black line and $A t h \_03 \_r n a 3$ is represented by a red line. Arrows indicate important peaks (linker lengths) in the smoothed distributions detected by Kernel Density Estimation.

Kurtosis and skewness measurements were calculated for each probability density plot and are shown in Table 4.7. Kurtosis gives an indication of the relative 'peakedness' of a distribution compared to the normal distribution, with positive values indicating a peaked distribution and negative values indicate a relatively flat (non-peaked) distribution. The kurtosis value for each dataset is negative, with the plot for the Ath_03_rna2 having a kurtosis of -0.91 , which is higher than the value for the Ath_03_rna3 plot (-1.53). This suggests that the Ath_03_rna2 smoothed distribution has more peaks, or a more important peak than the smoothed distribution of the Ath_03_rna3 dataset. The skewness measurement gives an indication of the symmetry of a distribution around the mean. A positive value indicates a skewed distribution with a tail extending towards positive values (higher than the mean) and a negative value indicates a skewed distribution with a tail extending towards negative values (lower than the mean). The smoothed distributions of both datasets Ath_03_rna2 and Ath_03_rna3 have positive values indicating the distributions are skewed with more values higher than the mean. The skewness value for the Ath_03_rna2 is higher than that for the Ath_03_rna3 distribution, indicating that the distribution of the Ath_03_rna2 has more data points with values higher than the mean (i.e. is more skewed towards higher values). 
Table 4.7 Kurtosis and skewness values for the smoothed distributions of linker lengths from the Ath_03_rna2 and Ath_03_rna3 datasets.

\begin{tabular}{|c|c|c|}
\hline Dataset & Kurtosis & Skewness \\
\hline Ath_03_rna2 & -0.91 & 0.70 \\
\hline Ath_03_rna3 & -1.53 & 0.30 \\
\hline
\end{tabular}

To determine whether there are significant differences in the distributions of linker lengths from datasets Ath_03_rna2 and Ath_03_rna3, a Kolmogorov-Smirnov test was used. The cumulative distribution function for each distribution was calculated, and the difference between the cumulative distributions at each linker length was determined. The $D$-statistic, which is the largest difference between the cumulative distributions, was 0.1019. This is larger than the critical $D$-value, indicating that the distributions of linker length for datasets Ath_03_rna2 and Ath_03_rna3 are significantly $(\mathrm{p}<0.05)$ different. The differences between the cumulative distributions were above the critical value for all linker lengths between $34 \mathrm{bp}$ and 116 bp. The cumulative distributions are shown in Figure 4.13.

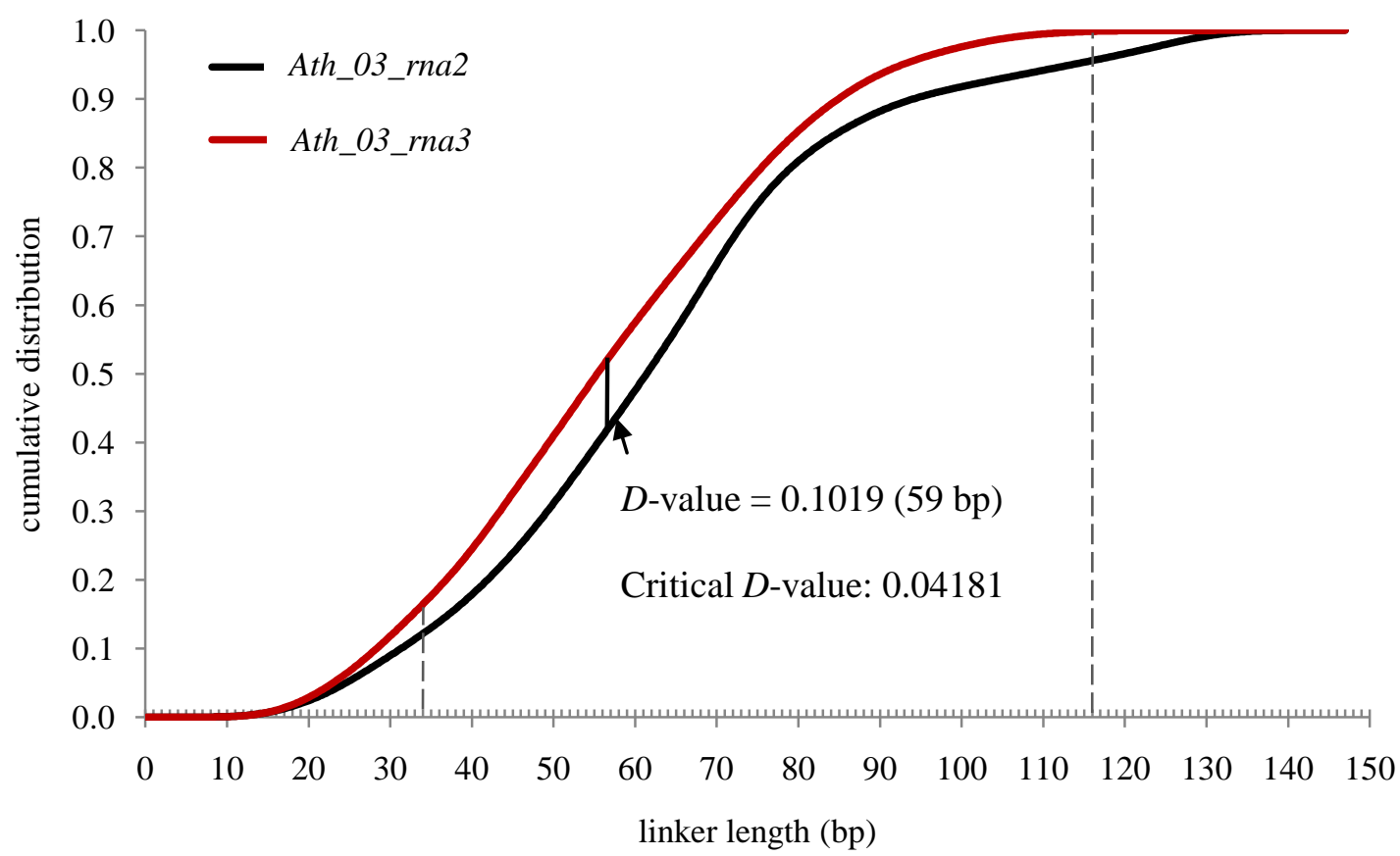

Figure 4.13 Cumulative distribution function plot for datasets Ath_03_rna2 and Ath_03_rna3. The value of the Kolmogorov-Smirnov $D$-statistic is indicated by a solid vertical black line. The cumulative distributions are significantly different at all linker lengths between the dashed lines (between linker lengths from $34 \mathrm{bp}$ and $116 \mathrm{bp}$ ). 


\subsection{Discussion}

\subsubsection{Arabidopsis linker length distribution}

Linker length was estimated from Arabidopsis dinucleosome sequences by subtracting twice the length of the nucleosome core (147 bp). Steric hindrance between nucleosomes is thought to impose constraints on linker lengths at short distances from the nucleosome, with linker lengths of up to 5bp, and from 10 to 15 bp thought to be unfavourable (Mengeritsky and Trifonov, 1983). Other, more recent studies, consider $10 \mathrm{bp}$ as the minimum linker length (Segal et al., 2006, supplementary data). However, there appears to be no agreement regarding minimum linker length in the literature.

In this study, linker lengths were calculated from the positions of mononucleosomes for the Ath_02_wt,Ath_04_MET1 and the C. elegans data sets. These linker lengths represent the relative distance between mononucleosomes from mapped positions of individual nucleosomes rather than the direct measurement of the physical distance between two nucleosomes, as derived from a dinucleosome dataset. Therefore, the mapped positions cannot be as reliable an indicator of linker length as linker length datasets derived from dinucleosome DNA fragment lengths. However, calculation of linker lengths from mapped mononucleosome positions has been successfully used to estimate linker lengths in yeast (Lubliner and Segal, 2009). Linker length was used as a measure of inter-nucleosome interactions, which were incorporated into a nucleosome prediction model. The distribution of derived yeast linker lengths showed a 10 bp periodicity, which had previously been demonstrated in yeast chromatin (Wang et al., 2008). This suggests that linker lengths derived from mapped mononucleosome positions are likely to be representative of in vivo linker lengths.

Linker lengths displayed a non-random distribution, in accordance with previous observations (Lohr and Van-Holde, 1979, Kato et al., 2003, Cohanim et al., 2005). The distributions of linker lengths from the Ath_03_wt and Ath_04_MET1 derived from mononucleosomes did not show the same 'peaked' distributions as the other datasets. This may be due to under-sampling for the Ath_01_wt, Ath_04_MET1 (dinucleosome) and Ath_02_wt datasets, which are therefore relatively small. The 
Nucleosome spacing and linker length variation differences between distributions, in terms of periodicity, could be tested by using a large number ( 1000) of random subsets of the data (linker lengths) from each dataset. The periodicity for each subset would then be calculated to provide a 'bootstrap' (Effron and Tibshirani, 1994) mean periodicity and 95\% CL, for each distribution. Non-overlapping CLs would then indicate statistically significant differences between the distributions.

To compare Arabidopsis linker lengths with those from other taxa, linker lengths were collated from the literature (Table 4.8). The mean linker lengths observed in this study for Arabidopsis wild-type were found to be between $52 \mathrm{bp}$ and $64 \mathrm{bp}$, with the exception of the Ath_03_wt dataset which had a mean of $40 \mathrm{bp}$. This suggests that Arabidopsis linker length falls closest to those calculated from human and chicken chromatin. The mean linker length for the Ath_03_wt dataset falls between those calculated from human and yeast chromatin. A general feature of the current and previous studies is considerable variation in the estimation of linker length from the same taxa. This may reflect variation associated with cell and tissue-specific expression patterns.

Table 4.8 A list of linker lengths for different species collected from literature.

\begin{tabular}{|c|c|c|c|}
\hline Organism & Tissue type/condition & $\begin{array}{l}\text { Mean linker } \\
\text { length }(\mathrm{bp})\end{array}$ & Reference \\
\hline C. elegans & & 68 & Johnson $e$ al., 2006 \\
\hline B. taurus & calf brain, cortical neurone & 21 & Allan et al., 1984 \\
\hline Gallus spp. & erythrocyte & $58^{*}$ & Satchwell and Travers, 1989 \\
\hline Gallus spp. & erythrocyte & $68 * *$ & Satchwell and Travers, 1989 \\
\hline H. sapiens & K562 cells & $28 *$ & Kato et al., 2003 \\
\hline H. sapiens & K562 cells & $48 * *$ & Kato et al., 2003 \\
\hline H. sapiens & - & 38 & Kahamann and Rake, 1993 \\
\hline H. sapiens & Down's syndrome & 26 & Kahamann and Rake, 1993 \\
\hline $\begin{array}{l}\text { Kluyveromyces } \\
\text { lactis }\end{array}$ & - & 24 & Heus et al., 1993 \\
\hline M. musculus & bulk chromatin & 48 & Cioffi et al., 2006 \\
\hline M. musculus & liver & 25 & Cioffi et al., 2006 \\
\hline Rattus spp. & pre-natal brain & 53 & Jaeger and Kuenzle, 1982 \\
\hline Rattus spp. & post-natal brain & 23 & Jaeger and Kuenzle, 1982 \\
\hline $\begin{array}{l}\text { Tetrahymenia } \\
\text { spp. }\end{array}$ & telomeres & 7 & Cohen and Blackburn, 1998 \\
\hline S. cerevisiae & grown to lag phase & $44 * * *$ & Yuan et al., 2005 \\
\hline
\end{tabular}

*Calculated from all DNA fragments

**Calculated from DNA fragments greater than $294 \mathrm{bp}$

*** Calculated from nucleosome positions defined using tiling arrays 
Nucleosome spacing and linker length variation

Previous studies have demonstrated differences in linker length between cells types exhibiting different transcriptional activity (Grigoryev et al., 1990). As the data presented here are generated from many cell types (within an organ), it is likely that multiple chromatin conformations are represented.

\subsubsection{Periodicity with linker length distribution in Arabidopsis}

The periodicity observed in the distribution of Arabidopsis linker lengths is different from the reported 10 bp periodicity for yeast and human chromatin (Widom, 1992, Kato et al., 2003, Wang et al., 2008). However linker lengths of multiples of $7 \mathrm{bp}$ have been reported previously for reconstituted nucleosomes with short linkers (Noll et al., 1980). The Arabidopsis anti-sense MET1 did exhibit the $\sim 10$ bp periodicity in the linker length distribution. This suggests that the overall chromatin structure of Arabidopsis anti-sense MET1 may be different from wild-type (by chromatin remodelling). DNA methylation has been shown to both inhibit and promote nucleosome formation in human and mouse imprinting control regions (Davey et al., 2003). Studies of chromatin conformation in mouse embryonic stem cells which lack Dnmt3a and DNmt3b showed an altered binding of linker histone (Gilberts et al., 2007). This revealed that linker histones are less mobile (or more-tightly bound) in methylation-deficient mouse cells. This is thought to be due to increased binding in the linker rather than the nucleosome. However, the reduced DNA methylation was not thought to alter chromatin compaction.

It has previously been suggested that the discrepancy between the periodicity of linkers obtained from dinucleosome sequences and the $10 \mathrm{bp}$ repeat is an artefact of the method of dinucleosome DNA fragment preparation (Cohanim et al., 2005). If the periodicity detected is indeed due to an experimentally induced artefact, and the true value is around $10 \mathrm{bp}$, then the discrepancy is likely to be seen in every case where the method has been implemented. This was not the case when investigating the linker length variation in Arabidopsis, human and C. elegans. It is therefore likely that the distributions of linker length and the periodicities they exhibit are a true reflection of the state of the chromatin at the time of nucleosome extraction. Any deviations from the proposed $10 \mathrm{bp}$ periodicity are likely to arise from structural changes in chromatin conformational brought about by chromatin remodelling. It is particularly interesting that when the Arabidopsis genome is de-methylated $(<20 \%$ 
Nucleosome spacing and linker length variation wild-type levels), the periodicity in linker length distribution returns to the previously reported $10 \mathrm{bp}$. This suggests that there may be a preferred 'default' setting for the positioning of nucleosomes, which is over-ridden by other epigenetic processes in wild-type (functional) chromatin.

\subsubsection{Relationship between linker length and intron length}

Previous in vivo studies and computational analysis of DNA sequences have shown that nucleosomes are likely to be positioned at 5'intron/exon3' boundaries (Denisov, et al., 1997). The results of these studies suggest that nucleosomes are positioned with the nucleosome dyad axis $0 \mathrm{bp}$ to $15 \mathrm{bp}$ from the intron/exon boundary, positioning the one half of a nucleosome within the intron and the other half in the exon. If nucleosomes are indeed positioned at 5'intron/exon3' boundaries, it may be expected that the length of the 5'intron/exon3' unit would reflect the nucleosome repeat length $(\mathrm{NRL}=$ one nucleosome and one linker). In this study, the distribution of intron length was compared to the distribution of nucleosome linker length. The distribution of the 5'intron-exon3' has a mean length of $322 \mathrm{bp}$, which does support the expectation, although the exon and intron length distributions have mean lengths of $170 \mathrm{bp}$ and $165 \mathrm{bp}$ respectively, which is consistent with the length of a nucleosome core and one molecule of histone H1 ( 165 -167 bp). This may suggest that nucleosomes are positioned more often within either the exon, or intron regions, rather than with the nucleosome dyad positioned at the 5 'intron/exon3' boundary as previously suggested. However the mean GC content of Arabidopsis introns is lower than that observed in Arabidopsis nucleosome core sequences (Chapter 3, Section 3.3.2.ii), which suggests that nucleosomes are positioned more often within exonic DNA.

To investigate nucleosome positioning at 5'intron/exon3' boundaries further, the periodicity in the distributions of the exon, intron and 5'intron/exon3' lengths was determined. The distribution of intron length has a periodicity of $7.87 \mathrm{bp}$, which is close to the periodicity of Arabidopsis linker length determined in this study, whereas the 5 'intron-exon3' dataset has a periodicity of $8.33 \mathrm{bp}$. This suggests that nucleosomes may be positioned more often in exon regions, with introns occurring more often in linker regions. 
The first introns relative to the 5' end of a transcriptional unit have been shown to have properties which distinguish them from subsequent introns in Arabidopsis. This includes them having longer length (Bradnam and Korf, 2008), and motifs which can promote and inhibit gene expression (Chung et al., 2006). The first intron length in Arabidopsis has a mean of $253 \mathrm{bp}$ and a median of $155 \mathrm{bp}$. The periodicity in the distribution of first intron length was 7.57, which is slightly lower than the periodicity in the distribution of all intron lengths. Although nucleosome positioning may be different at first introns compared to subsequent introns, this was not supported by the periodicity in the length distribution determined in this study. It would be interesting to compare specific histone modifications to nucleosome occupancy at first and subsequent introns in different length classes in order to investigate this further.

\subsubsection{Effect of linker length on higher-order chromatin structure}

Electron microscopy of chromatin fibres reconstituted from ordered nucleosome arrays possessing linkers of known lengths has shown that linker length variation dramatically influences the conformation of higher-order chromatin structure (Robinson et al., 2007). From these experiments, it appears that the diameter of the $30 \mathrm{~nm}$ chromatin fibre is related to linker length in a non-linear fashion. Chromatin fibres consisting of nucleosomes with $30 \mathrm{bp}$ to $60 \mathrm{bp}$ of linker DNA tend to have a diameter of $\sim 35 \mathrm{~nm}$, whereas fibres consisting of nucleosome with $70 \mathrm{bp}$ to $90 \mathrm{bp}$ of linker DNA tend to have a diameter around $45 \mathrm{~nm}$. These measurements were used to model the trajectory of the linker DNA within the $30 \mathrm{~nm}$ chromatin fibre for each linker length (Wong et al., 2007). The most energetically favourable conformation was described for each linker length from $30 \mathrm{bp}$ to $90 \mathrm{bp}$, resulting in seven different chromatin fibre conformations. A comparison of the modelled chromatin conformations is reproduced (Wong et al., 2007), and displayed along with the linker length distribution of Arabidopsis anti-sense MET1 in Figure 4.14. There appears to be a correspondence between the proposed chromatin fibre structures and the peaks in the linker length distribution of the Ath_04_MET1 dataset. 
The Wong et al. (2007) model suggests a shortest permissible linker length of $30 \mathrm{bp}$, with chromatin fibres having linker lengths between $30 \mathrm{bp}$ and $60 \mathrm{bp}$ having a fibre diameter of $35 \mathrm{~nm}$. It also suggests that there is a change in chromatin fibre diameter between $60 \mathrm{bp}$ and $70 \mathrm{bp}$ from $35 \mathrm{~nm}$ to $45 \mathrm{~nm}$. In the distribution of Ath_04_MET1 linker length, there appear to be relatively few linkers between $62 \mathrm{bp}$ and $63 \mathrm{bp}$, with $60.5 \%$ of the distribution lying between $30 \mathrm{bp}$ and $59 \mathrm{bp}$. This may reflect the relative distribution of chromatin fibre-class in Arabidopsis anti-sense MET1. A further $25.5 \%$ of the Ath_04_MET1 linker lengths fall between $60 \mathrm{bp}$ and $90 \mathrm{bp}$, which correspond to the $45 \mathrm{~nm}$ chromatin fibre in the Wong et al. (2007) model (with the remaining 14.0\% accounting for values 0-29 bp and 91-147 bp in length). This analysis suggests that the majority of chromatin in Arabidopsis anti-sense MET1 is organised into chromatin fibres of $\sim 35 \mathrm{~nm}$ in diameter.

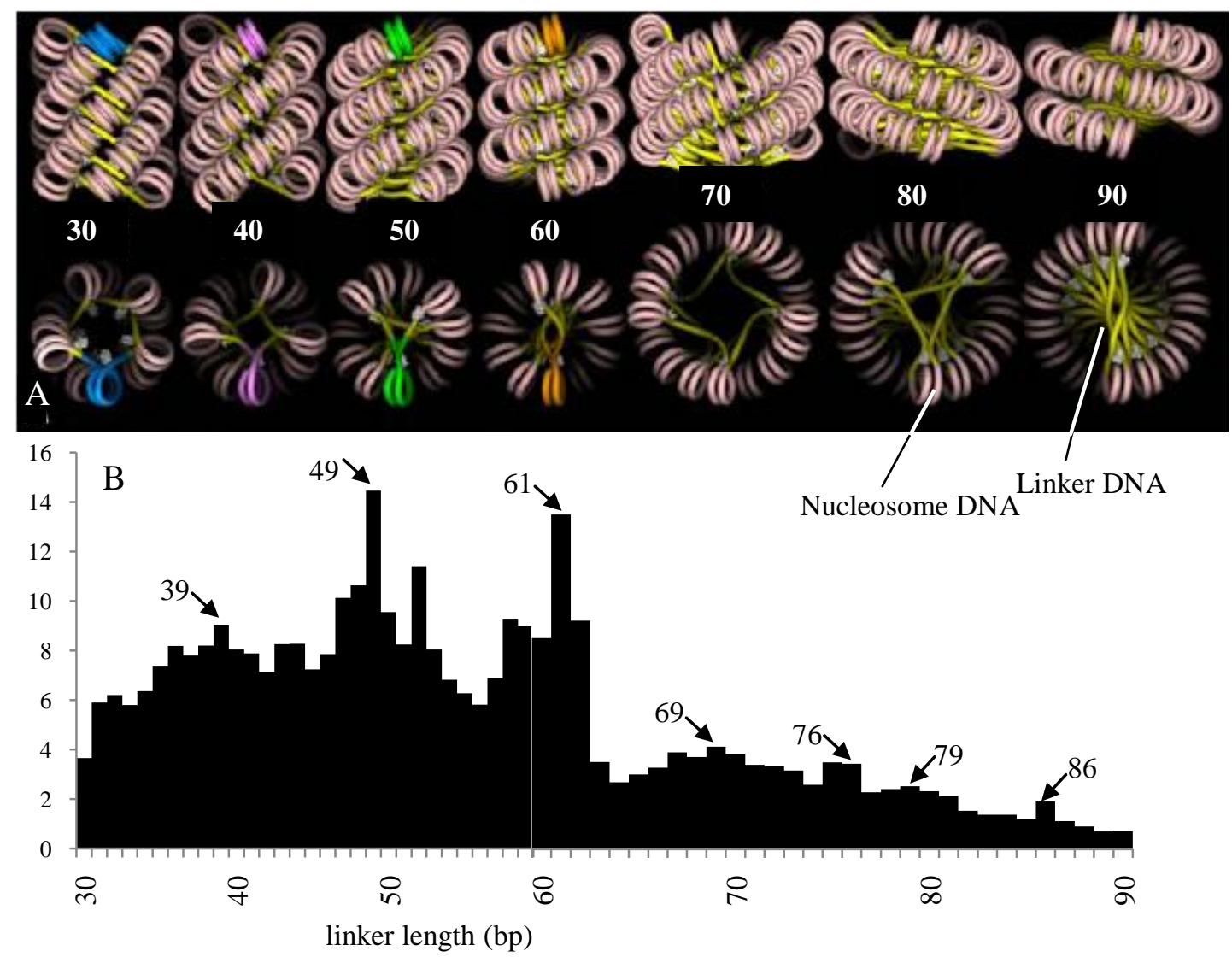

Figure 4.14 Demonstration of the possible relationship between A: models of the structure of the $30 \mathrm{~nm}$ fibre at increasing linker lengths (reproduced from Wong et al., (2007)) and B: Arabidopsis linker length calculated from the dinucleosome sequences of the Ath_04_MET1 dataset. The $x$-axis shows the frequency of each length (x 100). Each linker length is aligned to the chromatin proposed structure for that linker length. Peaks in the linker length distribution which approximately correspond to different chromatin conformations in the Wong et al. (2007) model are indicated. 
Nucleosome spacing and linker length variation

The differences within the Arabidopsis distributions are not as apparent for any of the Arabidopsis wild-type datasets, which may be a feature of the different sample sizes of the datasets.

The Wong et al. (2007) model of the $30 \mathrm{~nm}$ fibre structure assumes a linker histone (H5 rather than $\mathrm{H} 1$ in this model) at each nucleosome, although other studies suggests that this is not the case for many higher eukaryotes. The number of molecules of linker histone per nucleosome was found to range from 0.03 (yeast) to 1.3 (chicken) (Woodcock et al., 2006). The presence of linker histone (H1/H5) is thought to promote chromatin condensation, although the level of compaction is thought to be affected by linker length (Routh et al., 2008).

\subsubsection{Nucleosome positioning in the nucleolar organiser region in Arabidopsis}

The rDNA regions, on Arabidopsis Chromosomes 2 and 3, were chosen to investigate potential differences in linker length in regions with similar DNA sequence, since they were well represented in all datasets. It is likely that the large number of samples acquired is due to the $730 \pm 100$ rRNA repeats (NOR, chromosomes 2 and 4) which are not accounted for explicitly in the annotation of the Col-0 (TAIR v8) reference genome.

The rRNA genes have been shown to be associated with both active (euchromatic) and inactive (heterochromatic) chromatin marks in Arabidopsis (Lawrence et al., 2004). Bisulphite-treated rRNA promoters revealed that most are methylated, and chromatin immunopreciptitation (ChiP) revealed that methylated promoters were associated with $\mathrm{H} 3 \mathrm{~K} 9 \mathrm{me}^{2}$, a mark of heterochromatin. Conversely, hypomethylated promoters were associated with $\mathrm{H} 3 \mathrm{~K} 4 \mathrm{me}^{3}$ and Pol I, which indicated that those rRNA genes are active (Lawrence et al., 2004). With this in mind, the linker length distributions of the two rRNA regions were investigated to determine if two different chromatin structures could be detected. The two regions, represented in datasets Ath_03_rna2 and Ath_03_rna3 have different linker lengths: the Ath_03_rna2 distribution has a mean of $60 \mathrm{bp}$ and a median of $58 \mathrm{bp}$ while the Ath_03_rna3 distribution has a mean of $46 \mathrm{bp}$ and a median of $45 \mathrm{bp}$. This was supported by the Kolmogorov-Smirnov test for differences; the cumulative distributions of linker lengths from both datasets were statistically significantly different $(p<0.05)$ from 
Nucleosome spacing and linker length variation

each other. This suggests that the two regions are likely to have different chromatin structures and could reflect differences in the regulation of rRNA genes in the two regions.

\subsubsection{Structure of the rRNA regions on Chromosomes 2 and 3}

The positions of the 18S and 5S rRNA genes on Chr 2 and 3 were determined in this study using the annotation from the TAIR v8 Col-0 reference genome. The annotated rRNA genes on Chr 2 are most likely a single-copy representation of the nucleolar organiser region at the beginning of $\mathrm{Chr} 2$, which comprises of $730 \pm 100$ copies of the rRNA region (Wambutt et al., 2000). The 18S and 5S genes are also present in the centromeric region of $\mathrm{Chr} 3$. However this is thought to be just a single copy (European Union Chromosome 3 Arabidopsis Sequencing Consortium, 2000) and therefore, it does not seem likely that the large sampling of the nucleosome data represents the rRNA genes present on Chr 3. It may be that the data corresponds to copies of the rRNA genes in the NOR on Chr 4, as this region is absent in the annotated Arabidopsis reference genome (TAIR v8) and the sequence is almost identical (Wambutt et al., 2000). In addition, at the time of writing, the $25 \mathrm{~S}$ rRNA gene is also surprisingly still absent from the annotation of the reference genome (TAIR v8), although the sequence has been published (NCBI accession X52320, Unfried and Gruendler, 1991).

During the time the analyses of linker length distribution in the rRNA regions were performed, a new version of the Arabidopsis reference genome became available (TAIR v9, June, 2009), but the updated annotation still lacks the 25S, ITS, IGR and NOR4 regions. In addition, the updated reference genome also translocates the relative position of the annotated rRNA genes present on $\mathrm{Chr} 3$, but not the sequence, overall, so the new version is unlikely to affect the results of the linker length distribution analyses. Alignment of the published 25S rDNA sequence to the reference genome (TAIR v9) places the 25S rRNA gene alongside the 5S rRNA gene, and the ITS regions between the rRNA genes (Figure 4.15). Alignment of the intergenic region (IGR) alongside the rRNA genes was necessary as it also was not represented in the annotated reference genome. The Arabidopsis IGR sequence has 

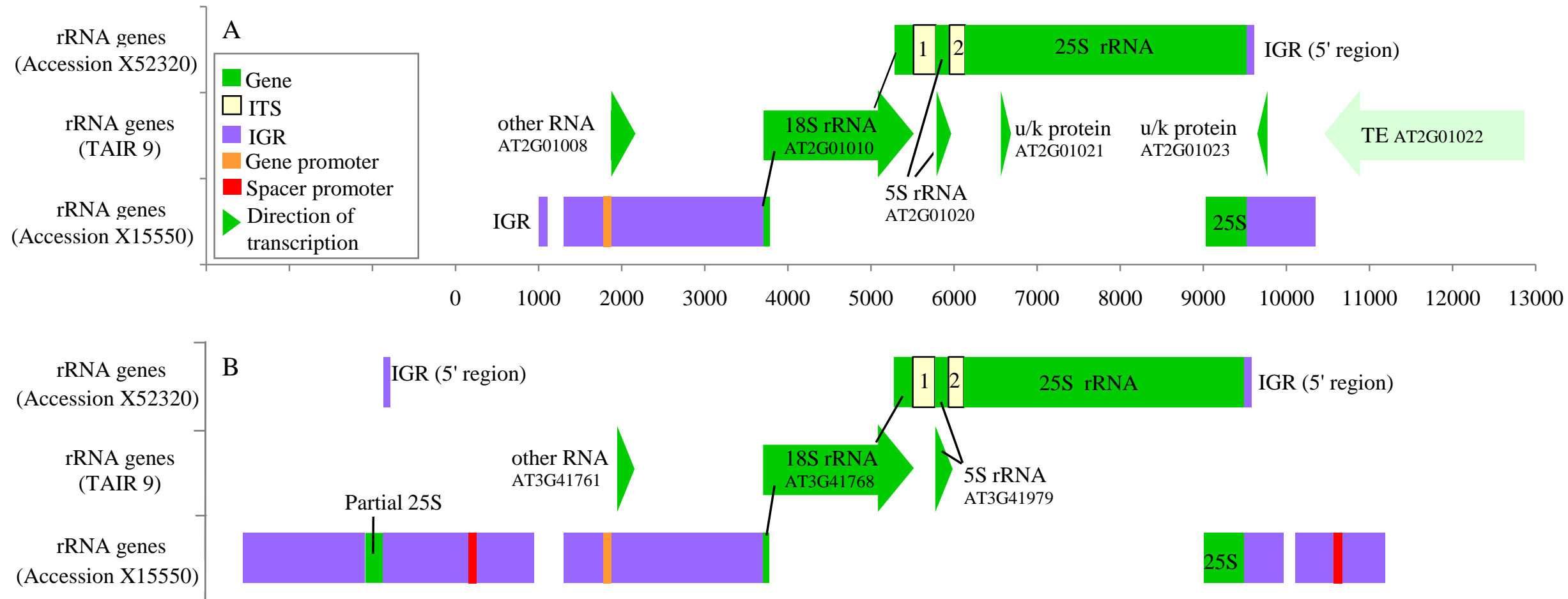

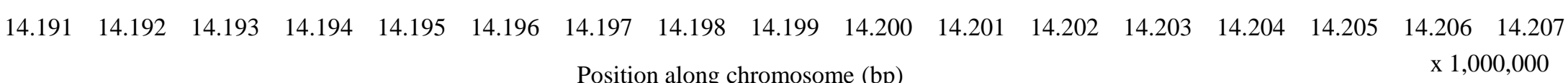

Figure 4.15 Structure of the annotated rRNA regions on A: Chromosomes 2, and B: Chromosome 3. Gene positions are shown in green, the ITS regions in yellow, IGR regions in purple, the gene promoters in orange and the spacer promoters in red. Accessions X15550 (Arabidopsis IGR) and X52320 (Arabidopsis ITS and 25S regions) were aligned to the Arabidopsis reference genome (TAIR v9) to position the non-annotated 25S, ITS1, ITS2 and IGR. In addition, the positions of the gene and spacer promoters are shown within the IGR. The transposable element on Chromosome 2 (AT2G01022) signals the end of the rDNA region in the annotated genome. The absence of the spacer promoter and some of the IGR from Chr 2 suggests that part of the sequence is missing from the annotation. 
Nucleosome spacing and linker length variation previously been published (NCBI accession X15550, Luschnig et al., 1993), and aligns partly upstream of the annotated rRNA gene positions, and partly downstream, which is unsurprising as it is part of the repeating unit and may signal the start and end of the rRNA region in the annotation. The gene and spacer promoter DNA sequences (Doelling et al., 1993) were also aligned (shown with the IGR positions, Figure 4.11), and this shows that part of the IGR containing the spacer promoter is missing from the annotated region on $\mathrm{Chr} 2$.

\subsubsection{Summary}

The distribution of linker length in Arabidopsis wild-type and anti-sense MET1 chromatin was investigated and found to be within a similar size range to that previously identified for other taxa. A periodicity of 7-8 bp was detected within the wild-type dinucleosome distributions which differed from the expected $\sim 10 \mathrm{bp}$ periodicity. Investigation of periodicity in the distributions of previously published nucleosome datasets revealed a period of $\sim 8$ bp in human chromatin and 5 bp in $C$. elegans chromatin, which were also different from the expected $10 \mathrm{bp}$. A period of 9-10 bp was detected in the distribution of linker lengths from the Arabidopsis antisense MET1, suggesting that DNA methylation is affecting nucleosome positioning and higher-order chromatin structure. Similarity between the periodicity of linker length and intron length distributions were detected, which suggest there may be a relationship between nucleosome spacing and intron length. It is interesting that the average intron (165 bp) and exon (170 bp) lengths are both close to the size of a nucleosome and linker histone $(\sim 167 \mathrm{bp})$. Of particular interest is the distribution of linker length within the rRNA regions. Dinucleosome sequences which map to either Chr 2 or Chr 3 were used to investigate region-specific chromatin structure in wild-type chromatin. This revealed two subsets with different distributions in linker length, possibly reflecting differences in higher order chromatin structure and gene regulation over the NORs. Unfortunately this analysis was not possible to perform with data from the anti-sense MET1. The Ath_O4_MET1 dataset consists mostly of mononucleosome DNA fragments and the extensive sampling over the rRNA regions resulted in only overlapping mononucleosome sequences, making linker estimation extremely difficult. 


\section{Chapter 5}

Nucleosome distribution and patterns of occupancy 


\subsection{Introduction}

Recent advances in high-throughput, technologies have massively increased the size and number of nucleosome datasets, making possible, concurrent with this thesis, study of genomic organisation patterns of nucleosome occupancy. Large-scale sequencing of nucleosome DNA fragments, and hybridisation of DNA to tiling arrays, in yeast and C. elegans, have in the last $2-3$ years revealed that nucleosomes have a non-random genomic distribution, and tend to be positioned more often within the coding regions, whilst intergenic regions are relatively nucleosome depleted (Lee et al., 2007; Johnson et al., 2007; Yuan et al., 2005).

In recent studies, the role of nucleosome positioning in the promoter regions has attracted much attention. Hybridisation of yeast mononucleosome DNA fragments to a high-resolution, overlapping tiling array revealed a nucleosome-free region (NFR), approximately 150 bp in length, commencing $200 \mathrm{bp}$ upstream from annotated genes (Yuan et al., 2005). NFRs in the regions upstream of the transcriptional start site (TSS) have since been found in a variety of organisms including Drosophila (Mavrich et al., 2008a), C. elegans (Johnson et al., 2007) and human (Schones et al., 2008; Ozsolak et al., 2007). NFRs are thought to occur at all promoters in the yeast genome, regardless of transcriptional activity, and this is thought to reflect a 'poised for transcription' chromatin state (Yuan et al., 2005). In contrast, the Drosophila genome does not appear to have NFRs throughout the genome. Thus genes with the promoter motifs: TATA, Inr or MTE, lack the NFR (Mavrich et al., 2008a).

NFRs have been found to be flanked on either side by a well-positioned nucleosome within genes transcribed by Pol II (Yuan et al., 2005). Furthermore, these flanking nucleosomes were found to contain the histone variant H2A.Z in yeast (Albert et al., 2007), whereas H2A.Z was only found within nucleosomes of highly transcribed genes in Drosophila (Marvich et al., 2008a). In addition, the H2A.Z-containing nucleosomes adopted a more ordered pattern of organisation than bulk nucleosomes, and are thought to confer a more open chromatin structure. 
Nucleosome distribution and patterns of occupancy

For transcription to occur, a pre-initiation complex, comprising of the transcription factor, Pol II and activator proteins is bound at the promoter prior to transcription. Current evidence suggests that to allow for binding of the pre-initiation complex, the positioned nucleosome upstream of the TSS is either re-positioned or removed (Workman, 2006).

\subsubsection{Aims of study in this chapter}

Whilst much effort has been put into study of nucleosome organisation around the promoter/transcriptional start site, and to some extent the 5' and 3' ends of genes and the mechanism of transcription, the occupancy around the exon, intron and intergenic regions have been neglected. As the positioning around the TSS varies between yeast and Drosophila, it is likely that species-specific patterns of nucleosome organisation may exist both within the transcriptional units and in their 5' and 3' regions.

With this in mind, the following hypotheses were constructed and tested:

1. Nucleosomes are evenly distributed throughout the Arabidopsis genome.

2. Biases in nucleosome occupancy differ between coding and non-coding regions in Arabidopsis.

3. Ordered positioning of nucleosomes exists around the TSSs in Arabidopsis, comparable to the organisation found within other taxa.

4. The Arabidopsis Nucleolar Organiser Regions display different nucleosome patterns of positioning dependent on chromosomal origin, due to the transcriptionally heterogeneous nature of these regions.

5. Overall differences in nucleosome positioning exist between wild-type and the Arabidopsis anti-sense MET1. 


\subsection{Materials and methods}

\subsubsection{Genomic representation in datasets and distribution of fragments}

To determine the distribution between the chromosomes of fragments from each dataset, the percent of each chromosome represented in the nucleosome datasets is given by:

$\mathrm{n}=\left(\frac{F_{(C h r x)}}{F_{(t o t a l)}}\right) \times 100$

Where:

$n=\%$ represented each chromosome represented within the dataset

$F_{(C h r)}=$ fragments within the dataset which align to Chr $x$

$F_{(t o t a l)}=$ total number of fragments within the dataset

To determine the percent of nucleosome-occupied bases on each chromosome, the number of unoccupied bases was determined calculated by subtracting the start coordinate of $n \_k+1$ from the end co-ordinate of $n \_k$ (Figure 5.1) to give the distance between occupied bases: nuc $\_k_{(\text {end })}-$ nuc_ $k+1_{\text {(start }}$.

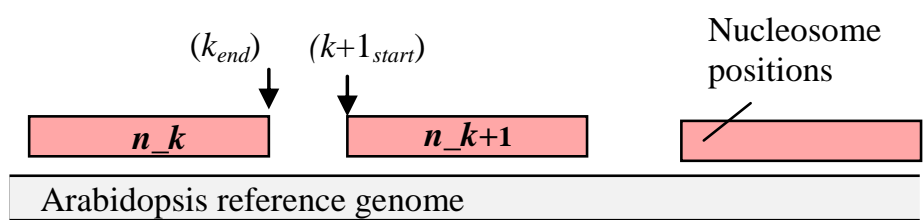

Figure 5.1 Schematic showing the method for calculating the distance between the positions of nucleosome fragments aligned to the Arabidopsis reference genome.

The percent occupancy was calculated using the following equation:

$\%$ occupancy $=100-\left(\left(\frac{\sum b_{(d>0)}}{b_{(t o t a l)}}\right) \times 100\right)$

Where:

$b_{(d>0)}=$ distance between occupied bases, summed over all the gaps between fragments

$b_{(t o t a l)}=$ total number of bases in the chromosome. 
Nucleosome distribution and patterns of occupancy To investigate the distribution of nucleosomes within chromosome 1, the frequency of the start co-ordinate of each nucleosome DNA fragment was calculated in bin sizes of $10,000 \mathrm{bp}$. A similar operation was performed to investigate a $2 \mathrm{Mbp}$ region with a 1,000 bp bin size.

\subsubsection{Genomic patterns of nucleosome occupancy}

To begin to understand patterns of nucleosome occupancy in Arabidopsis, nucleosome occupancy scores were calculated (Chapter 2, section 2.2.vi). A count of nucleosome molecules occupying any given co-ordinate was determined, and an occupancy value assigned to each co-ordinate (Figure 5.2). The nucleosome occupancy scores were initially viewed using a prototype genome browser developed at Rothamsted by Graham King.

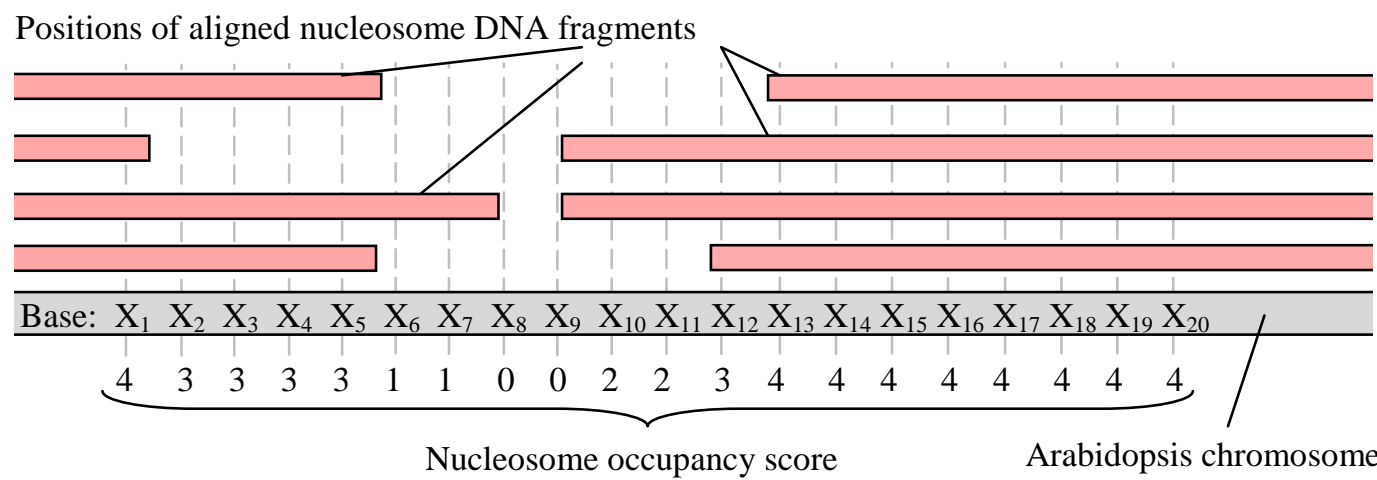

Figure 5.2 Schematic showing the assignment of nucleosome occupancy scores to each position within the Arabidopsis chromosome.

\section{$\underline{\text { 5.2.3 Distribution of occupancy within exons and introns }}$}

Annotation classes were assigned to each position, along with the nucleosome occupancy score as described in Chapter 2, section 2.2.vi.

For exons, the average occupancy (number of molecules) per base was calculated using the equation:

occupancy/bp $=\frac{\sum\left(b_{(1 . . n)} \times o_{(1 . . n)}\right)_{(\text {exon })}}{B_{(\text {total exon })}}$

Where:

$b=$ number of bases (in exon category);

$o=$ number of molecules (occupancy); 
Nucleosome distribution and patterns of occupancy

$B($ total exon $)=$ number of bases in Arabidopsis nuclear genome DNA (in exon

category).

The exon/intron ratios were calculated for nucleosome occupancy, using the formula:

$\operatorname{ratio}_{(\text {exon })}=\frac{\text { Occupancy } / \text { bp }(\text { exon })}{(\text { Occupancy } / b p)_{(\text {exon })}+(\text { Occupancy } / b p)_{(\text {intron })}}$

\section{$\underline{\text { 5.2.4 Nucleosome occupancy around transcriptional start sites }}$}

In order to determine the presence and occupancy of nucleosomes around the TSS in Arabidopsis, nucleosome DNA fragment co-ordinates were compared to a set of published, experimentally determined and predicted TSS (Tanaka et al., 2009).

In order to determine what percent of TSS was occupied by at least one nucleosome, the distance between the TSS and nearest upstream nucleosome boundary was calculated by subtracting the co-ordinate of the TSS position from the nucleosome end-position (Figure 5.3), where distances between 0 and 147 (bp) indicate that the TSS is occupied.

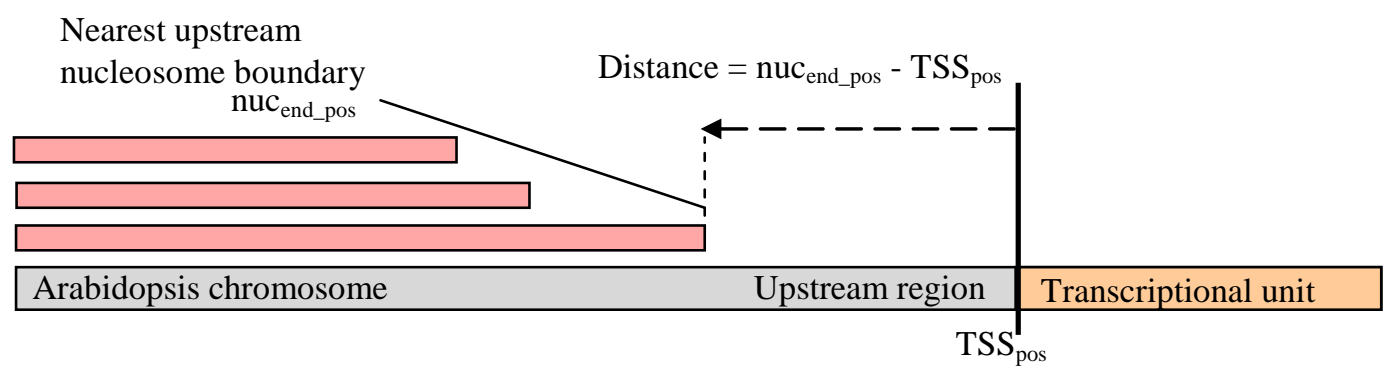

Figure 5.3 Schematic showing the method for determination of the distance between the TSS and the nearest upstream nucleosome boundary.

In order to determine the nucleosome occupancy within the vicinity of the TSS, the distance between the TSS and the nucleosome boundary was calculated by subtracting the midpoint of every nucleosome position from the TSS (where the nucleosome midpoints are positioned to within 1000 bp of the TSS) (Figure 5.4). 


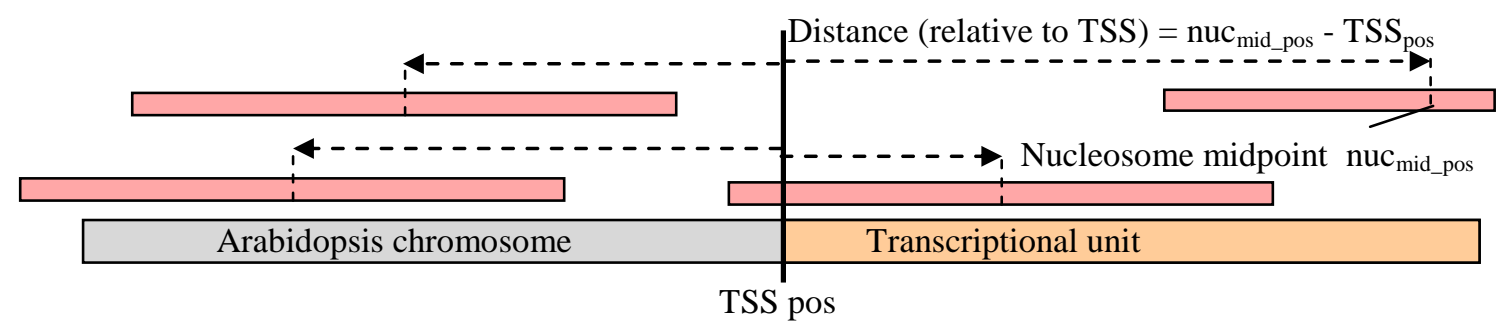

Figure 5.4 Schematic showing the method for determination of the distance between the TSS and every nucleosome midpoint within $1000 \mathrm{bp}$.

\subsubsection{Kernel Density Estimation}

In order to inspect the frequency of nucleosome occupancy in the vicinity of the TSS for evidence of ordered nucleosome positioning, Kernel Density Estimation was used. This smoothed the occupancy frequency distribution around the TSS and is able to reveal longer-range peaks in the distributions. The density estimations were performed in GenStat ${ }^{\circledR}$ (2008) with the bandwidth calculated using the method of Sheather \& Jones (1991), and using a set of 2,048 grid points. 


\subsection{Results}

\section{$\underline{5.3 .1 \text { Genomic distribution and coverage of nucleosome sequences }}$}

In order to determine if the nucleosome sequences of datasets Ath_03_wt Ath_04_MET1 and Ath_05_wt are distributed evenly across the genome, the number of sequences which align to each chromosome was calculated and expressed as a percent of the total sequences. The percent of the genome contained within each chromosome was also calculated for comparison and is shown in Table 5.1. Both Chrs 2 and 3 appear to be over-represented in the Ath_05_wt dataset. Chr 3 is also over-represented in the Ath_04_MET1 dataset. This is most likely because of the large number of nucleosome DNA fragments which align to the rRNA genes annotated on Chrs 2 and 3.

Table 5.1 Percent of sequences from datasets Ath_03_wt, Ath_04_MET1 and Ath_05_wt which align to each chromosome. The percent of the genome on each chromosome is shown for comparison.

\begin{tabular}{r|rrrr}
\hline Chr & Genome & Ath_03_wt & Ath_04_MET1 & Ath_05_wt \\
\hline 1 & 25.53 & 24.67 & 24.15 & 22.99 \\
2 & 16.53 & 16.52 & 16.47 & 18.01 \\
3 & 19.69 & 21.49 & 22.67 & 22.57 \\
4 & 15.59 & 15.35 & 15.56 & 15.07 \\
5 & 22.65 & 21.97 & 21.14 & 21.37 \\
\hline
\end{tabular}

The number of bases occupied by nucleosomes was calculated for each chromosome and expressed as a percentage of the total number of bases for each chromosome, for the datasets Ath_03_wt Ath_04_MET1 and Ath_05_wt (Table 5.2). Occupancy is lower for all chromosomes in the Ath_05_wt dataset than the other two. Presence of linker DNA in the Ath_03_wt may falsely increase the number of occupied bases due to the wider coverage of each molecule.

Table 5.2 Percent of occupied bases for each chromosome for the datasets Ath_03_wt, Ath_04_MET1 and Ath_05_wt. Values for the total genome are highlighted in bold.

\begin{tabular}{l|rrrrrr}
\hline $\begin{array}{l}\text { Occupied in } \\
\text { each set }\end{array}$ & Chr 1 & Chr 2 & Chr 3 & Chr 4 & Chr 5 & Total \\
\hline Ath_03_wt & 66.04 & 66.05 & 68.80 & 67.52 & 66.89 & $\mathbf{6 7 . 0 1}$ \\
Ath_04_MET1 & 63.86 & 62.88 & 64.38 & 63.26 & 63.76 & $\mathbf{6 3 . 6 8}$ \\
Ath_05_wt & 48.96 & 50.55 & 52.71 & 51.34 & 50.06 & $\mathbf{5 0 . 5 8}$ \\
\hline
\end{tabular}


In order to determine the distribution of nucleosome sequences within each chromosome, the 5' (start) co-ordinate of each nucleosome DNA fragment was plotted, using a bin size of $10,000 \mathrm{bp}$. The distribution of nucleosome sequences from datasets Ath_02_wt, Ath_03_wt and Ath_04_MET1 is shown in Figure 5.5. There appear to be some differences between the wild-type plots and the plot of antisense MET1 positions around the centromere region of chromosome 1 (region in the grey box, Figure 5.5). To investigate this further, the co-ordinates of the nucleosome start sites were plotted over a region from $14,000,000$ bp to $16,000,000 \mathrm{bp}$ using a $1,000 \mathrm{bp}$ bin size, as shown in Figure 5.6. In the expanded view of the 2,000,000 bp region, there appears to be little difference between the wild-type and anti-sense MET1 nucleosome start co-ordinates at this scale. Any differences observed appear to be associated with the relative abundance of nucleosome sequences in each bin. 

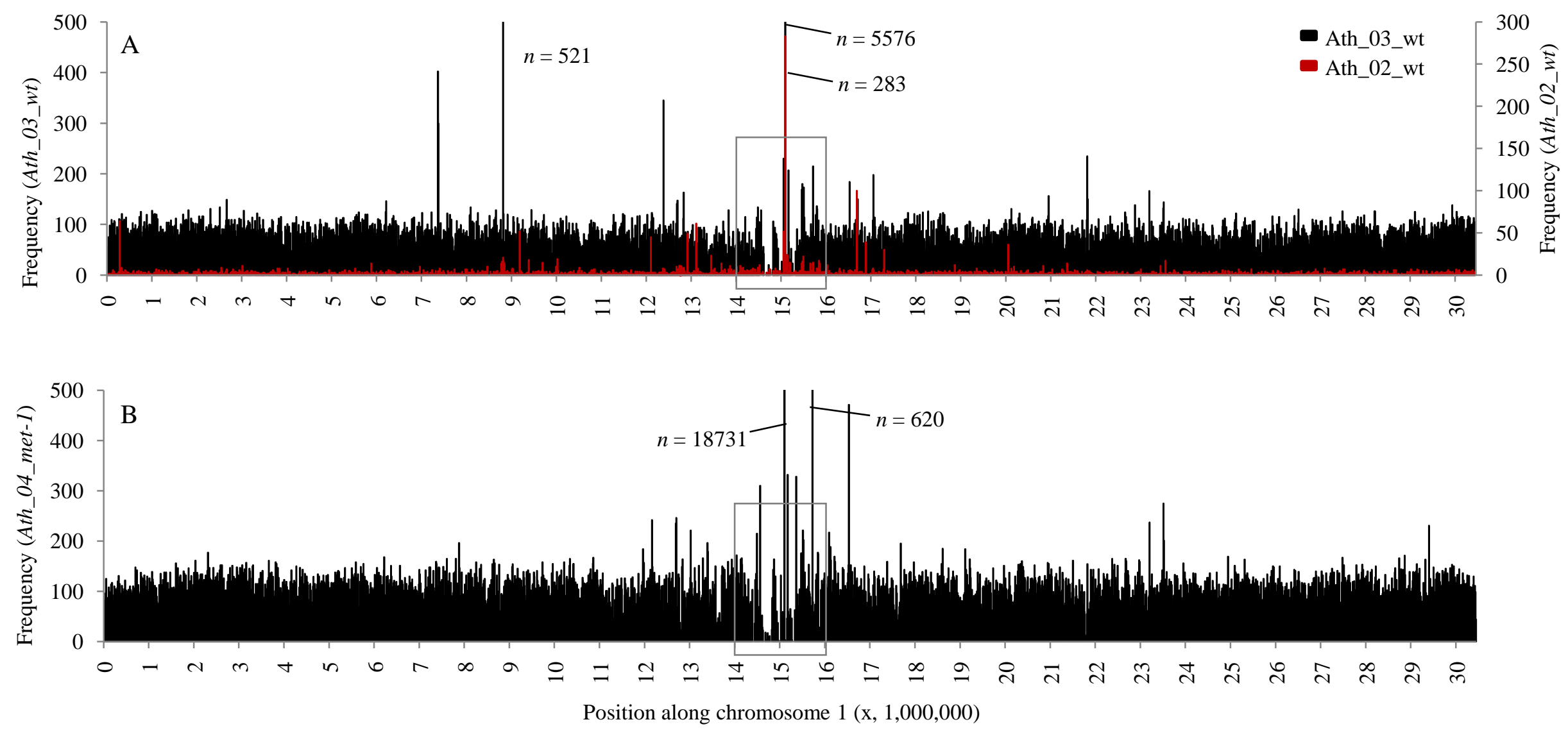

( 5.5 The density of nucleosome sequence fragment start co-ordinates within chromosome 1 for datasets A: Ath 02 wt, Ath 03 wt and B:Ath 04 plotted with a $\mathbf{1 0 , 0 0 0}$ bp bin size. For clarity, the Ath 02 wt dataset is plotted on a separate $y$-axis. The region in the grey box indicates some differences between the Ath_03_wt and Ath_04_MET1 datasets, which are plotted at higher resolution. Figure 5.6. 

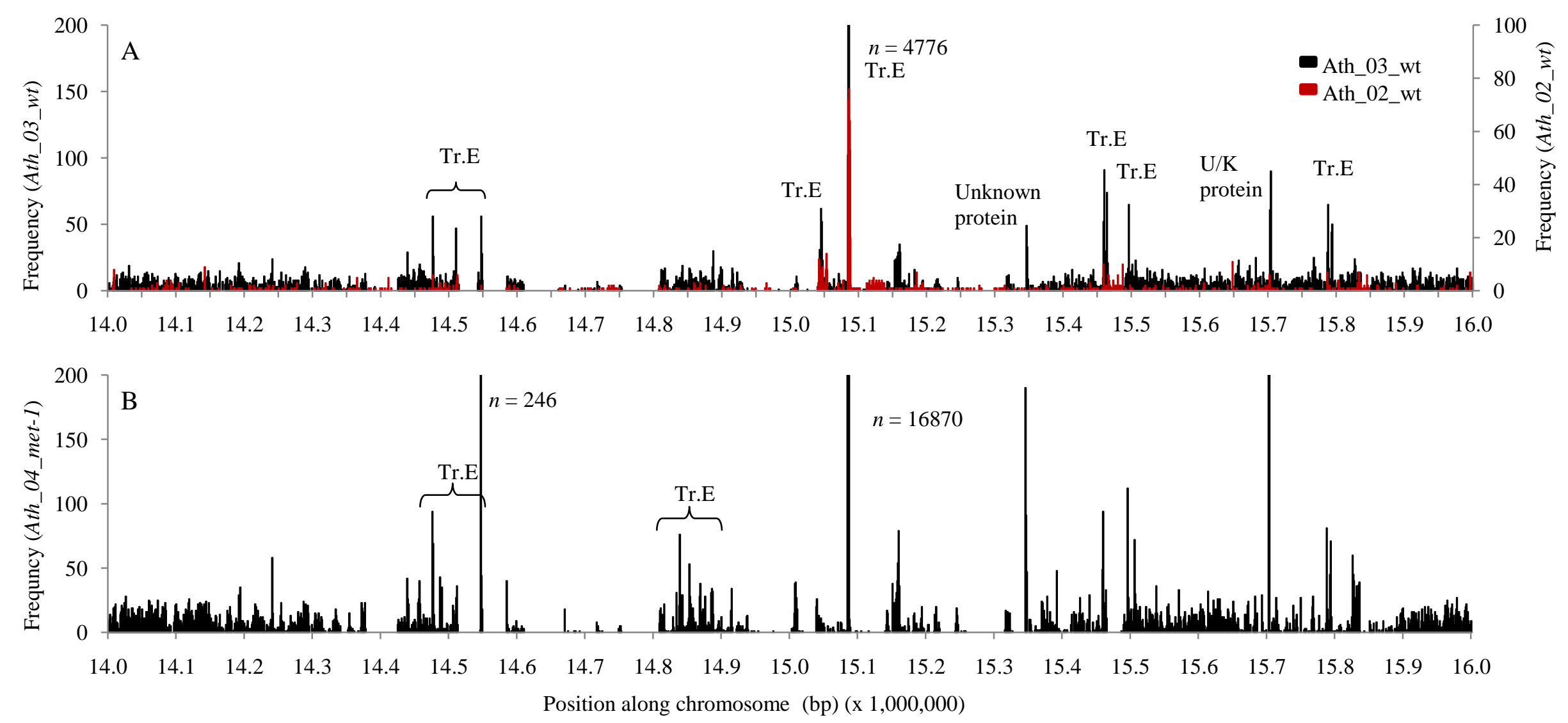

Figure 5.6 The density of nucleosome sequence fragment start co-ordinates within a close-up of a 2 Mbp region (boxed in Figure 5.5), within chromosome 1 for datasets A: Ath_02_wt, Ath_03_wt and B:Ath_04_MET1, plotted with a 1,000 bp bin size. For clarity, the Ath_02_wt dataset is shown on a separate $y$-axis. Where peaks are observed, the corresponding gene descriptions at those co-ordinates are shown. 


\section{$\underline{\text { 5.3.2 Trends of nucleosome occupancy }}$}

Mapped nucleosome occupancies were viewed using a prototype genome browser, developed by Graham King, Rothamsted Research (data not shown). This revealed differences in nucleosome positioning between the Ath_03_wt and Ath_04_METI datasets. Two variables affect these datasets: mononucleosome vs. dinucleosome and anti-sense MET1 vs. wild-type. Therefore, to understand and ascribe any observed differences to one or other variable, a further comparable wild-type mononucleosome sequence dataset was generated. However this was only available towards the very end of the project. The nucleosome DNA which was used to produce this dataset originated from the same sample (and same MNase digest) as the material from which the Ath_03_wt dataset was produced.

\subsection{2.i Genomic trends of nucleosome occupancy}

The nucleosome occupancy of datasets Ath_03_wt, Ath_04_MET1 and Ath_05_wt was compared, and a few general trends in nucleosome positioning emerged. There are many cases for datasets Ath_03_wt, Ath_04_MET1 and Ath_05_wt where the peak in nucleosome occupancy is high over a relatively long stretch of DNA (500 $700 \mathrm{bp}$ ) (spread), indicating conserved, de-localised (or fuzzy) positioning. In contrast, high peaks in the nucleosome occupancy with a narrow spread occur, indicating conserved well-positioned nucleosomes. In addition, there are regions of lower occupancy throughout the genome and some regions which appear to be nucleosome-free. For the Ath_03_wt and Ath_05_wt datasets, the majority of nucleosome occupancy appears to be within the transcriptional units, with the intergenic regions generally having a lower occupancy, or being nucleosomedepleted. Overall, nucleosome positioning tends to be more regularly spaced in the Ath_04_MET1 dataset, with increased occupancy within the intergenic region when compared to the wild-type.

\section{$\underline{\text { 5.3.2ii Nucleosome occupancy in specific chromosomal regions }}$}

To understand further some of the properties and potential functional consequences of differences in nucleosome occupancy observed between the datasets, two regions on chromosome 1 were chosen for further investigation: one, containing the imprinted gene PHERES1 (co-ordinates 24,258,093 - 24,271,483 bp) and two, a 
Nucleosome distribution and patterns of occupancy

region containing a cluster of genes controlling floral development (co-ordinates $25,985,494-26,013,322 \mathrm{bp})$. The genes present in each region along with a brief description are shown in Tables 5.3 and 5.4.

Table 5.3 Gene models present in a selected region (1) on chromosome 1

\begin{tabular}{l|lrrrrl}
\hline Gene model & $\begin{array}{l}\text { Gene } \\
\text { name }\end{array}$ & Chr & $\begin{array}{l}\text { TAIR 8 start } \\
\text { co-ordinate }\end{array}$ & $\begin{array}{l}\text { TAIR 8 stop } \\
\text { co-ordinate }\end{array}$ & Strand & Description \\
\hline AT1G65300 & $\begin{array}{l}\text { PHERES 2 } \\
\text { (AGL38) }\end{array}$ & 1 & $24,258,592$ & $24,259,428$ & $\mathrm{~F}$ & MADS box TF \\
AT1G65310 & ATXTH17 & 1 & $24,260,879$ & $24,262,136$ & $\mathrm{~F}$ & $\begin{array}{l}\text { Xyloglucan } \\
\text { endotransglucosylase/ } \\
\text { hydrolase }\end{array}$ \\
AT1G65320 & N/A & 1 & $24,263,551$ & $24,266,614$ & $\mathrm{R}$ & $\begin{array}{l}\text { CBS-domain, } \\
\text { unknown function }\end{array}$ \\
AT1G65330 & $\begin{array}{lllll}\text { AHERES 1 } \\
\text { (AGL37) }\end{array}$ & 1 & $24,270,144$ & $24,270,983$ & $\mathrm{R}$ & $\begin{array}{l}\text { MADS box, imprinted, } \\
\text { regulated by MEA and } \\
\text { FIE }\end{array}$ \\
\hline
\end{tabular}

Table 5.4 Gene models present in a selected region (2) on chromosome 1

\begin{tabular}{l|lccccl}
\hline Gene model & $\begin{array}{l}\text { Gene } \\
\text { name }\end{array}$ & Chr & $\begin{array}{l}\text { TAIR 8 start } \\
\text { co-ordinate }\end{array}$ & $\begin{array}{l}\text { TAIR 8 stop } \\
\text { co-ordinate }\end{array}$ & Strand & Description \\
\hline AT1G69120 & AP1 & 1 & $25,985,993$ & $25,989,976$ & R & $\begin{array}{l}\text { MADS box, floral } \\
\text { homeotic gene }\end{array}$ \\
AT1G69150 & N/A & 1 & $25,997,322$ & $25,998,875$ & F & $\begin{array}{l}\text { DC1 domain-containing } \\
\text { protein }\end{array}$ \\
AT1G69160 & N/A & 1 & $26,003,877$ & $26,005,251$ & F & Unknown protein \\
AT1G69170 & SPL6 & 1 & $26,008,731$ & $26,010,926$ & F & $\begin{array}{l}\text { TF, petal differentiation } \\
\text { and expansion }\end{array}$ \\
AT1G69180 & CRC & 1 & $26,011,128$ & $26,012,722$ & R & $\begin{array}{l}\text { Carpel and style } \\
\text { development, floral } \\
\text { meristem ID }\end{array}$ \\
\hline
\end{tabular}

The 5' and 3' ends of the genes within region 1 showed differences in the nucleosome occupancy between the three datasets (Figure 5.7). The red arrows indicate differences between the two wild-type datasets and the Ath_04_MET1 dataset at the 5' boundaries of PHERES1 and PHERES2. Since nucleosome occupancy at the 5' and 3' regions is known to be important for transcriptional regulation, this suggests that these genes may be subject to different transcriptional 
Nucleosome distribution and patterns of occupancy regulation in the anti-sense MET1. This region also demonstrates the presence of more regularly spaced nucleosomes within the Ath_O4_MET1 dataset. For example, in Figure 5.7 black arrows indicate nucleosome present at positions in the Ath_04_MET1 dataset, which are absent from the other two datasets Ath_03_wt and Ath_05_wt. Regions of high occupancy with a wide spread, representing fuzzy positioning of nucleosomes (arrow 1) contrast with regions of high occupancy and narrow spread representing well-ordered positioning of nucleosomes (arrow 2). In addition, a nucleosome free region is observed between the PHERES 2 and AT1G65310 genes.

Differences in nucleosome occupancy within the 5' and 3' genic regions are also observed within region 2 (indicated by red arrows, Figure 5.8). However, there were also differences at the 5' and 3' ends between the Ath_03_wt and Ath_05_wt datasets in this example. The black arrows indicate occupancy in the Ath_04_MET1 dataset which is not observed in the Ath_03_wt and Ath_05_wt datasets. The differences observed in this example all lie within the intergenic regions. In both the region 1 and 2 examples, the higher nucleosome occupancy peaks tend to be consistent with the positions of the exons. In addition, where defined, the 5' and 3' UTRs tend to have low nucleosome occupancy or are nucleosome-depleted. 


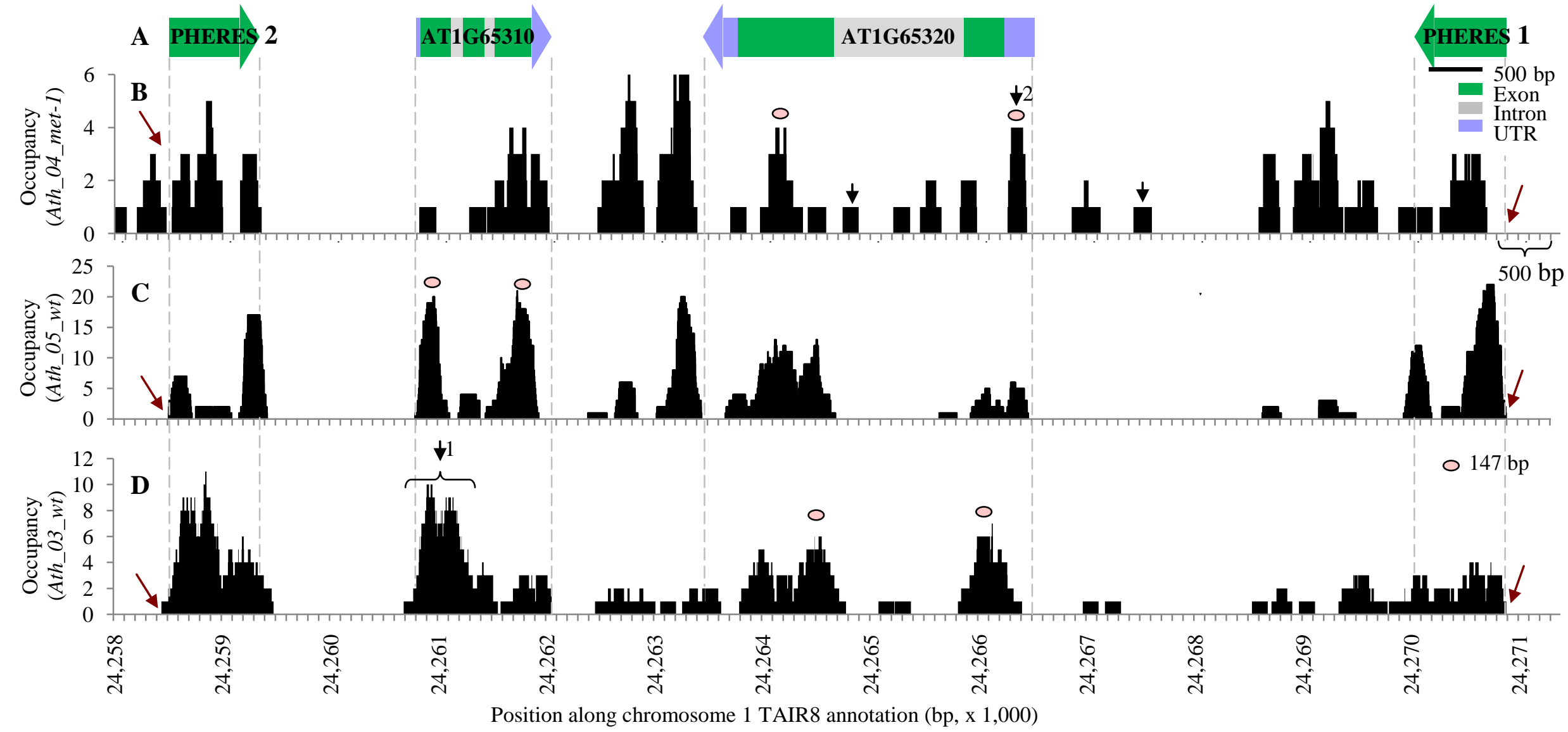

Figure 5.7 Nucleosome occupancy of datasets B: Ath_04_MET1 C: Ath_03_wt, and D:Ath_05_wt, over a 14,500 bp region (region 1) within chromosome 1, containing the imprinted gene PHERES1 (A). Red arrows indicate 5' and 3' regions where the nucleosome occupancy differs between datasets. Regions of occupancy observed in Ath_04_MET1 which are not observed in the wild-type datasets are indicated with black arrows. For comparison, ellipses of nucleosome size are added. 

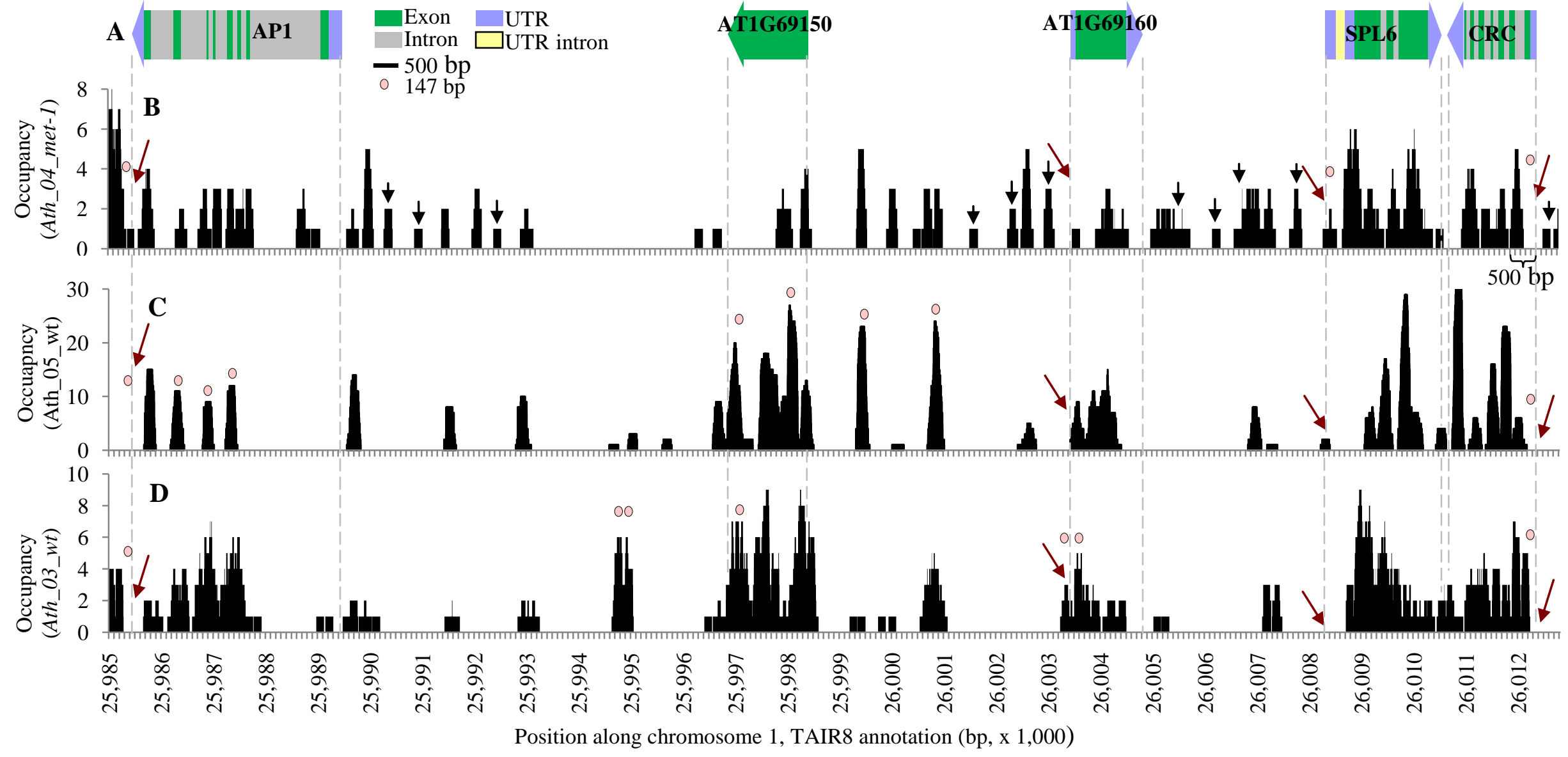

Figure 5.8 Nucleosome occupancy of datasets B: Ath 04_MET1 C:Ath 03 wt, and D:Ath 05 wt, over a 28,000 bp region (region 2) within chromosome 1 , containing floral homeotic genes $(\mathbf{A})$. Red arrows indicate 5 ' and 3 ' regions where the nucleosome occupancy differs between datasets. Regions of occupancy observed in Ath_04_METI which are not observed in the wild-type datasets are indicated with black arrows. For comparison, ellipses representing nucleosome size (147 bp) are added. 


\subsection{2.iii. Nucleosome occupancy within coding vs. non-coding regions}

Based on these general and specific observations, a more systematic analysis was carried out. In order to determine whether there is a bias in nucleosome occupancy between introns and exons in Arabidopsis, the ratios of occupancy within genic:intergenic and exonic:intronic DNA were calculated for different gene annotation classes for the datasets Ath_03_wt, Ath_04_MET1 and Ath_05_wt (Table 5.5). For comparison, the overall intron:exon ratios for each annotation class represented on Chr 1 were also calculated.

Table 5.5 Exon/intron ratios of nucleosome occupancy and average occupancy/base within different annotation classes on chromosome 1, for datasets the Ath_03_wt, Ath_04_MET1 and Ath_05_wt. Overall exon/intron ratios and \% of different gene annotation classes on Chr 1 are also shown. The average occupancy/bp and ratios of exon/intron are highlighted

\begin{tabular}{|c|c|c|c|c|c|c|c|c|}
\hline $\begin{array}{c}\text { Annotation } \\
\text { class }\end{array}$ & \multicolumn{2}{|c|}{ Ath_03_wt } & \multicolumn{2}{|c|}{ Ath_04_MET1 } & \multicolumn{2}{|c|}{ Ath_05_wt } & $\begin{array}{c}\text { Chr } 1 \\
\%\end{array}$ & $\begin{array}{l}\text { Chr } 1 \\
\text { ratio }\end{array}$ \\
\hline & Ratio & Occupancy & Ratio & Occupancy & Ratio & Occupancy & & \\
\hline Genic & 0.663 & 3.88 & 0.591 & 4.61 & 0.717 & 7.02 & 58.40 & 0.584 \\
\hline $\begin{array}{l}\text { Intergenic } \\
\begin{array}{r}\text { protein } \\
\text { coding: }\end{array}\end{array}$ & 0.337 & 1.97 & 0.409 & 3.20 & 0.283 & 2.77 & 41.60 & 0.416 \\
\hline Exon & 0.718 & 8.70 & 0.685 & 9.83 & 0.710 & 9.19 & 32.4 & 0.673 \\
\hline Intron & 0.282 & 3.42 & 0.315 & 4.51 & 0.290 & 3.75 & 15.8 & 0.327 \\
\hline 5'UTR exon & 0.551 & 3.56 & 0.543 & 4.41 & 0.673 & 5.22 & 3.87 & 0.847 \\
\hline 5'UTR intron & 0.449 & 2.89 & 0.457 & 3.72 & 0.327 & 2.54 & 0.700 & 0.153 \\
\hline 3'UTR exon & 0.561 & 3.57 & 0.526 & 4.22 & 0.699 & 5.29 & 3.43 & 0.835 \\
\hline $\begin{array}{l}\text { 3'UTR intron } \\
\text { Pseudogene: }\end{array}$ & 0.439 & 2.79 & 0.474 & 3.81 & 0.301 & 2.28 & 0.676 & 0.165 \\
\hline Exon & 0.527 & 3.89 & 0.562 & 4.42 & 0.656 & 8.33 & 0.583 & 0.964 \\
\hline $\begin{array}{l}\text { Intron } \\
\qquad \text { misc RNA }\end{array}$ & 0.473 & 3.49 & 0.438 & 3.44 & 0.344 & 4.38 & 0.0310 & 0.036 \\
\hline Exon & 0.569 & 4.56 & 0.499 & 3.98 & 0.688 & 6.61 & 0.0839 & 0.667 \\
\hline Intron & 0.431 & 3.46 & 0.501 & 3.99 & 0.312 & 3.00 & 0.0310 & 0.333 \\
\hline 5'UTR exon & 0.854 & 17.46 & 0.833 & 19.22 & 0.910 & 30.13 & 0.0855 & 0.305 \\
\hline 5'UTR intron & 0.146 & 2.98 & 0.167 & 3.86 & 0.090 & 3.00 & 0.0427 & 0.695 \\
\hline 3'UTR exon & 0.565 & 3.65 & 0.490 & 4.21 & 0.706 & 6.80 & 0.0652 & 0.661 \\
\hline $\begin{array}{r}\text { 3'UTR intron } \\
t R N A\end{array}$ & 0.435 & 2.81 & 0.510 & 4.38 & 0.294 & 2.83 & 0.0335 & 0.339 \\
\hline Exon & 0.575 & 5.32 & 0.489 & 4.88 & 0.184 & 7.19 & 0.0006 & 0.337 \\
\hline Intron & 0.425 & 3.93 & 0.511 & 5.10 & 0.816 & 31.81 & 0.0010 & 0.663 \\
\hline
\end{tabular}

The genic:intergenic ratios for the Ath_03_wt and Ath_05_wt indicate that the genic regions of chromosome 1 are occupied by nucleosomes more often than the intergenic regions. The genic/intergenic ratio calculated for the Ath_04_MET1 
Nucleosome distribution and patterns of occupancy dataset is lower than that for the Ath_05_wt for exons and is closer to the total genic/intergenic ratio, which suggests that the nucleosomes in the Arabidopsis antisense METI are more evenly distributed than in the wild-type and that the average nucleosome occupancy/ bp in the Ath_04_MET1 dataset is 3.2, compared to 2.7 in Ath_05_wt. In general, the exon:intron ratios indicate that exons are occupied by nucleosomes more often than introns in all datasets. The Ath_04_MET1 exon:intron ratios, while indicating more occupancy of exons than introns, are nearer 0.5. This suggests that there is a more even distribution of nucleosomes between introns and exons. The exon/intron ratios for the dataset Ath_03_wt tend to be nearer those calculated for the Ath_04_MET1 datasets. 


\section{$\underline{\text { 5.3.3 Nucleosome positioning around transcriptional start sites }}$}

Previous studies in the small yeast genome have indicated a nucleosome free region approximately $150 \mathrm{bp}$ in length, $200 \mathrm{bp}$ upstream from the transcriptional unit (or within the promoter region) (Yuan et al., 2005). In the absence of extensive promoter information for Arabidopsis, nucleosome presence at the transcriptional start sites was determined using a set of experimentally determined and predicted TSS (Tanaka et al., 2008).

\subsection{3.i Nucleosome presence at the transcriptional start site (TSS)}

The distances between the TSS and nearest upstream nucleosome were calculated for both the forward and reverse strands of chromosome 1 for the datasets Ath_03_wt and Ath_04_METI (Figure 5.9) and divided into three categories: those that are occupied, those which have the nearest nucleosome boundary within $200 \mathrm{bp}$ upstream and those which have the nearest nucleosome boundary more than $200 \mathrm{bp}$ upstream.

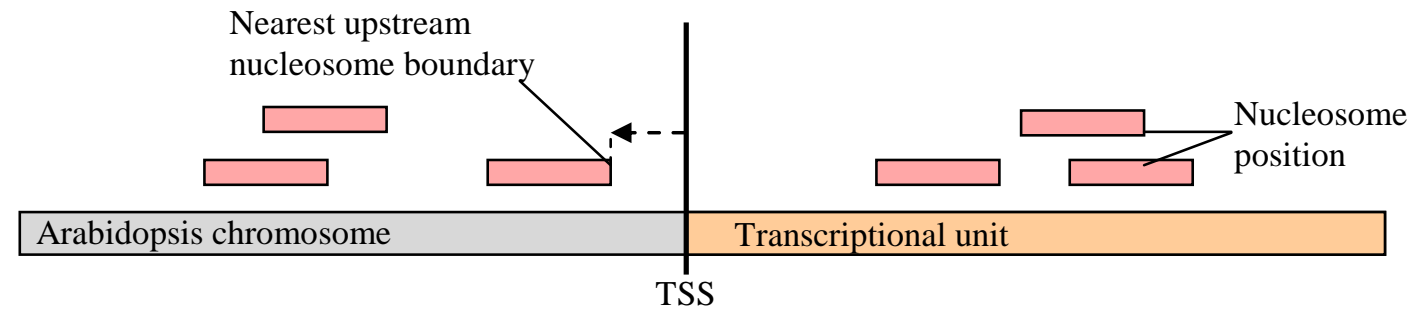

Figure 5.9 Schematic showing the method for determination of the distance between the TSS and the nearest upstream nucleosome boundary.

The percentage of TSS in each of the categories was calculated and is shown in Table 5.6. For both Ath_03_wt and Ath_04_MET1, $72 \%$ of the TSS were occupied by at least one nucleosome DNA fragment. In addition, for $\sim 17 \%$ of the TSSs, the nearest nucleosome boundary was within the region $200 \mathrm{bp}$ of upstream of the TSSs. For the remaining $\sim 11 \%$ of the TSSs, the distance between the nearest upstream nucleosome boundary and TSS was more than $200 \mathrm{bp}$. As many of the TSSs in this dataset are derive from a prediction algorithm, there are alternative TSSs for the same gene model within the dataset. The high occupancy of the TSSs may be due to alternative TSSs close to each other occupied by the same nucleosome fragment. In order to adjust for this, the categories were re-calculated using only one TSS for each 
gene model (the furthest upstream in each case). This did not appear to alter the occupancy estimation, with $72 \%$ TSS occupied over both the forward and reverse strands in the Ath_03_wt and Ath_04_MET1 datasets. In addition, there was little difference in the relative numbers of occupied TSSs between the datasets Ath_03_wt and Ath_04_MET1.

Table 5.6 The presence of nucleosomes at, and upstream of TSSs within chromosome 1 for the datasets: Ath_03_wt and Ath_04_MET1.

\begin{tabular}{|c|c|c|c|c|c|c|}
\hline \multirow[b]{2}{*}{ Category } & \multicolumn{3}{|c|}{ Ath_03_wt } & \multicolumn{3}{|c|}{ Ath_04_MET1 } \\
\hline & Forward & Reverse & All & Forward & Reverse & All \\
\hline all TSS & & & & & & \\
\hline Occupied & 72.77 & 72.05 & 72.40 & 72.77 & 72.15 & 72.46 \\
\hline 0 to $-200 \mathrm{bp}$ & 15.85 & 17.92 & 16.71 & 15.77 & 17.37 & 16.58 \\
\hline$-200+b p$ & 11.38 & 10.64 & 10.89 & 11.46 & 10.48 & 10.96 \\
\hline 1 TSS only & & & & & & \\
\hline Occupied & 70.15 & 73.08 & 71.62 & 70.15 & 73.14 & 71.65 \\
\hline 0 to $-200 \mathrm{bp}$ & 16.64 & 16.42 & 16.53 & 16.57 & 16.36 & 16.47 \\
\hline$-200+b p$ & 13.21 & 10.50 & 11.85 & 13.27 & 10.50 & 11.89 \\
\hline
\end{tabular}

\subsection{3.ii Nucleosome occupancy around transcriptional start sites}

In order to determine the nucleosome occupancy around the TSS, the midpoint of each nucleosome in the dataset Ath_05_wt was calculated. For all TSS within the first $6,000,000 \mathrm{bp}$ on the forward strand of $\mathrm{Chr} 1$, the distance between each nucleosome midpoint and each TSS was calculated (Figure 5.10).

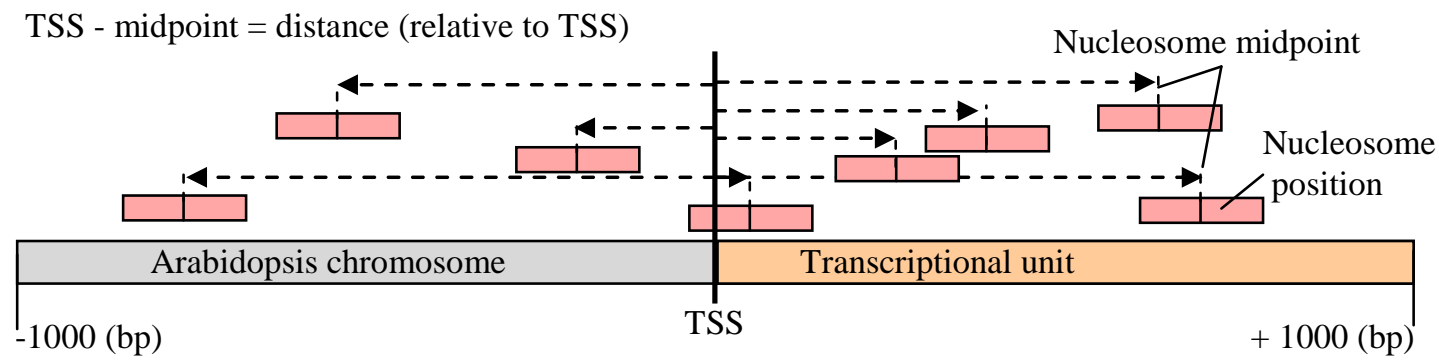

Figure 5.10 Schematic showing the method for calculating the frequency of nucleosome occupancy around the TSSs by subtracting the position of the TSS from the nucleosome midpoint.

For each TSS, the distance between the TSS and every nucleosome midpoint within $1000 \mathrm{bp}$ of the TSS was plotted against the position on Chr 1, to produce a density map (Figure 5.11.A). There appear to be regions within Chr 1 where the nucleosome 
occupancy is denser, specifically in the vicinity of co-ordinates $\sim 2 \mathrm{Mbp}, \sim 3 \mathrm{Mbp}, 4$ 4.5 Mbp and 5-6 Mbp. The regions of greater density appear to occur in the region to the right (immediately downstream) of the TSS. In addition, there are less dense regions of nucleosome occupancy around $1.5 \mathrm{Mbp}$ along the chromosome. The differences in nucleosome density could be due to differences in gene density. To test this, the density of the TSSs within 0-6 Mbp Chr 1 (10,000 bp bin size) is shown in Figure 5.11.B. The nucleosome depleted region around 1.5 Mbp does appear to coincide with a lack of TSS in this area. In comparison, the nucleosome-dense areas around $2 \mathrm{Mbp}$, 4-3 Mbp and 5-6 Mbp appear to co-incide with an increase in TSS frequency within these regions. In order to calculate the estimated occupancy of the TSS, a subset of the distances between each TSS and all nucleosomes within $73 \mathrm{bp}$ was produced. Of all the TSS in the region, $69.71 \%$ were occupied by nucleosomes. The frequency of nucleosome occupancy relative to the position of the TSS on Chr 1 is shown in Figure 5.11.C.

To test the hypothesis that specific patterns of occupancy exist around the TSS, the frequency of nucleosome midpoints for each position around the TSS was plotted (Figure 5.11.D). A peak in the distribution is observed 25-72 bp after (downstream) from the TSS. Nucleosome occupancy also appears to be greater downstream of the TSS compared with upstream, indicating that the region upstream may be nucleosome depleted or relatively nucleosome free, which is consistent with previous studies (Mavrich et al., 2008a;b; Yuan et al., 2005). In addition, there appears to be some periodicity in the distribution, which may indicate ordered positioning around the TSS. A periodicity of $10.05 \mathrm{bp}$ (CI 9.99-10.10 bp) was detected within the distribution by Fourier analysis. However this is likely to be due to periodicity with the distribution of alternate TSS. Kernel Density Estimation was used to reveal longer-range peaks within the distribution (Figure 5.12). 

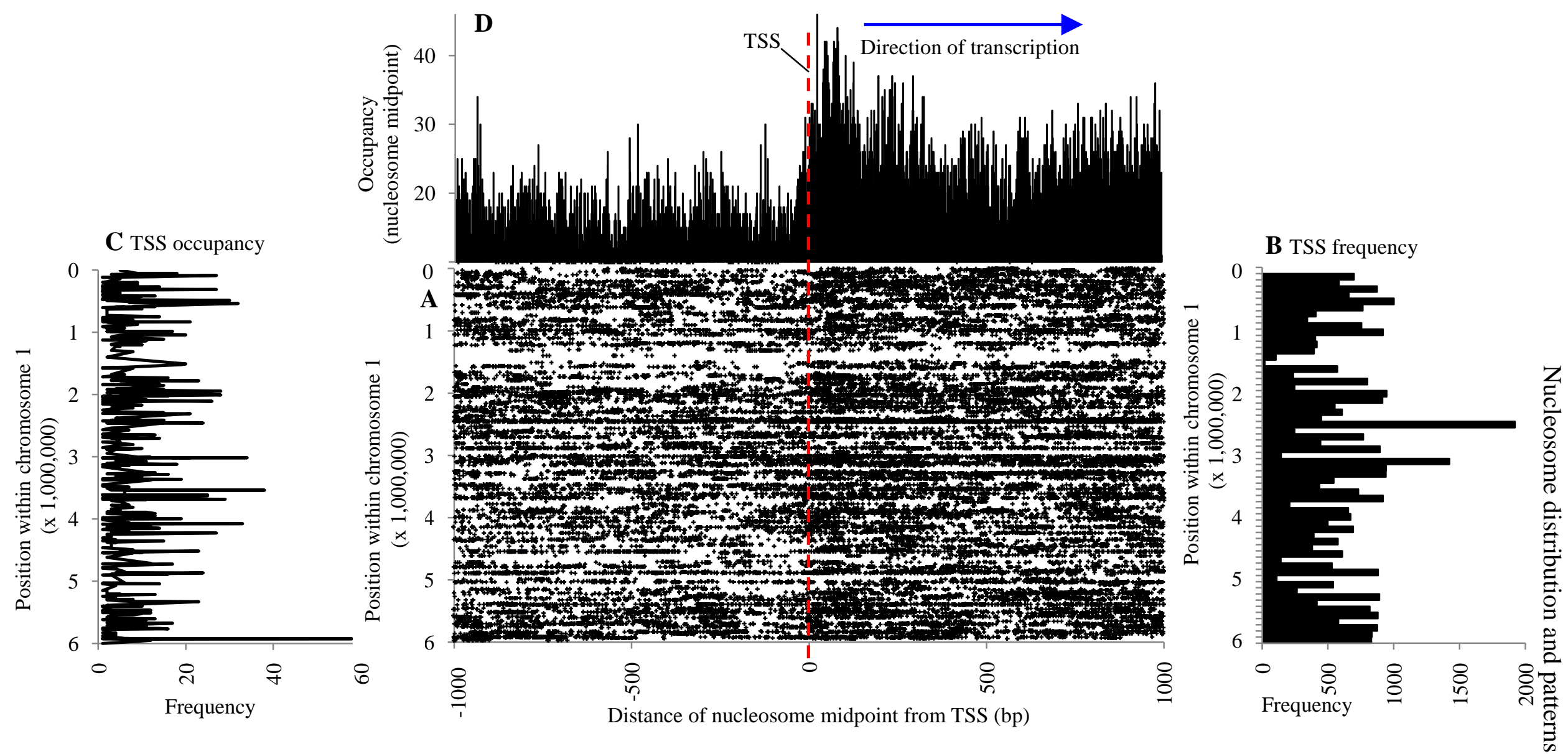

Figure 5.11 Nucleosome occupancy around the TSS of the first $6 \mathrm{Mbp}$ of the forward strand of chromosome 1. A: The distances of all nucleosomes within 1000 bp either side of the TSS relative to the position on Chr 1, B: the nucleosome occupancy of the TSS relative to the position within Chr 1, C: TSS density (relative to the position on Chr 1, and D: frequency of nucleosome occupancy at each position within $1000 \mathrm{bp}$ of the TSS. 


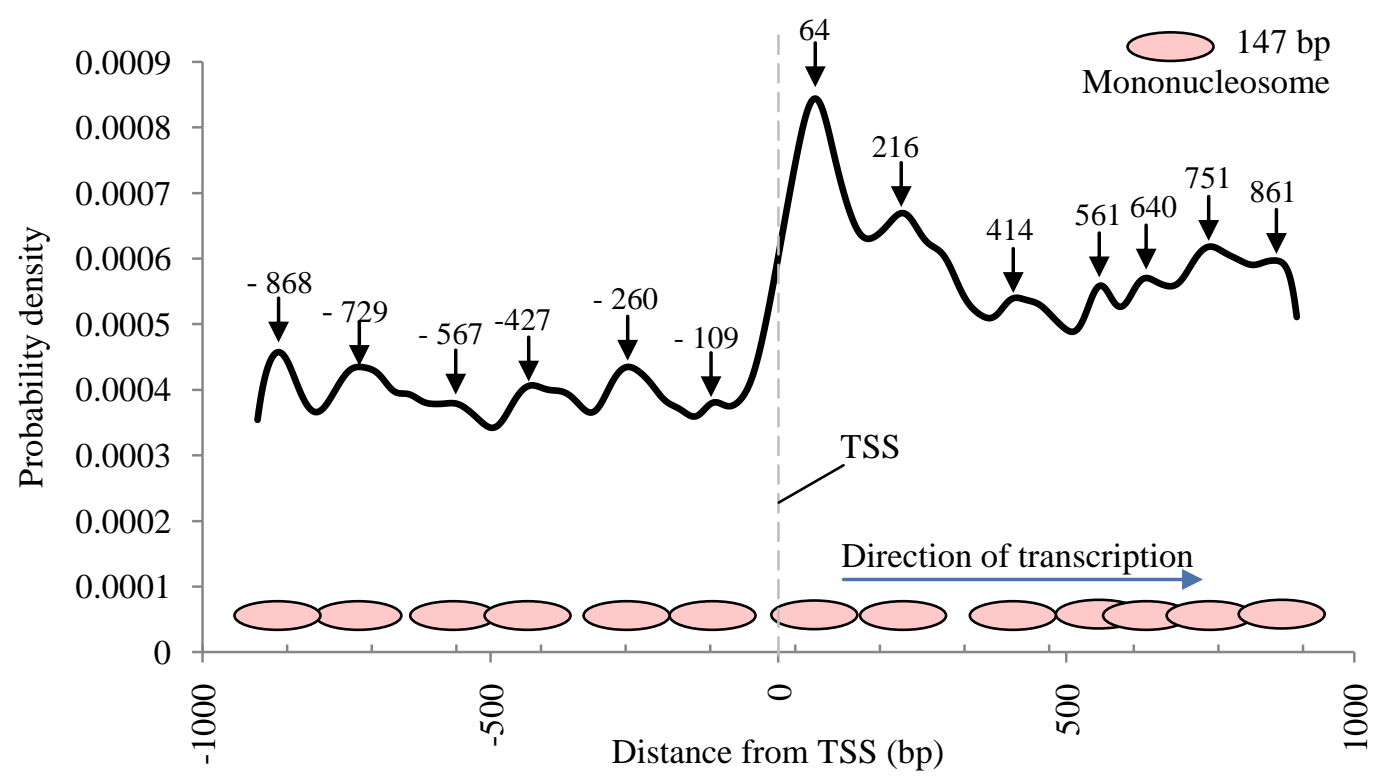

Figure 5.12 Kernel Density Estimation of nucleosome occupancy around the TSSs within the first $6 \mathrm{~Kb}$ of chromosome 1 (forward strand). The distance (bp) between the TSS and important peaks in the nucleosome occupancy are indicated. Internal $x$-axis markers are spaced at $147 \mathrm{bp}$ and ellipses representing nucleosome size are added for comparison.

Smoothing of the distribution revealed a peak in the nucleosome occupancy at +64 bp with respect to the TSS. This is consistent with $\sim 70 \%$ of the TSS being occupied by nucleosomes. Further peaks were observed in the smoothed distribution of nucleosome occupancy, both upstream and downstream of the TSS, which may indicate ordered positioning around the TSS. To investigate this further, the distance between the apex of each peak was determined and the mean distance was calculated to be $144.1 \mathrm{bp} \pm 9.2$ bp with a $95 \%$ CI of 123.9 - 164.4 bp for a sample of 12 distances. The calculation was repeated, but omitting the distances between peaks which occur after +500 bp with respect to the TSS, since these peaks appear to correspond to overlapping nucleosome positions within this region as such they may correspond to the end of ordered positioning (or correspond to another, overlapping signal $\sim 500 \mathrm{bp}$ from the TSS). The mean for this sub set is $158.7 \mathrm{bp} \pm 6.3 \mathrm{bp}$ with a $95 \%$ CI of $144.3-173.2 \mathrm{bp}$, for a sample of 9 distances. 


\subsubsection{Nucleosome occupancy around the Arabidopsis nucleolar organiser region}

The regions containing rRNA genes annotated on chromosomes 2 and 3 have different biases in linker length, indicating differences in chromatin structure between the two regions (Chapter 4, section 4.3.4). Again it should be noted that the sequence of Arabidopsis NORs on Chrs 2 and 4 are incomplete in the current reference genome. The sequence of a single repeat on $\mathrm{Chr} 3$ probably represents the 700-900 copies known to exist in the Chr 4 NOR. In order to test the hypothesis that differences exist in the nucleosome positioning and occupancy, which may affect the chromatin structure of the Arabidopsis NOR regions, the nucleosome occupancy from datasets Ath_03_wt, Ath_04_MET1 and Ath_05_wt around the homologous rRNA genes were aligned, and are shown in Figure 5.13.

There appear to be differences in the positioning and occupancy of the rRNA genes in all datasets. Most notably, a peak in the nucleosome occupancy around the 3' boundary of the 25S rRNA gene is observed within Chr 2, but is absent from the corresponding position on Chr 3 in all datasets, (indicated on Figure 5.13 by arrows 1,4 and 6). The nucleosome positioning over the 18S rRNA gene appears to be wellordered and conserved between Chr 2 and 3 for the dataset Ath_05_wt. However, there are interesting differences between $\mathrm{Chr} 2$ and 3 and the 5' and 3' boundaries of the 18S rRNA gene. A peak in the nucleosome occupancy within Chr 2 is observed approximately $150 \mathrm{bp}$ upstream of the 5' boundary of the 18S rRNA gene, which appears to be absent within Chr 3 (indicated by arrows 2, Figure 5.13). In addition, a further peak in the nucleosome occupancy is observed which appears to span the 3' edge of the 18S rRNA gene on Chr 3, which is absent from Chr 2 (indicated by arrows 3, Figure 5.13). Differences are also observed in the nucleosome positioning between Chr 2 and 3 in the Ath_03_wt dataset, over a region which spans the 5', boundary of the $18 \mathrm{~S}$ rRNA gene and approximately $350 \mathrm{bp}$ upstream. The occupancy of dinucleosomes at the 5' boundary of the 18S rRNA gene on Chr $2(n=$ 400 molecules) appears to be approximately half of that on Chr 3. However, the positioning on Chr 2 appears to be well-ordered with two well-defined peaks representing the two nucleosomes (indicated by arrows 5, Figure 5.13). The welldefined peaks are not observed in the corresponding position on Chr 3. 
Nucleosome distribution and patterns of occupancy

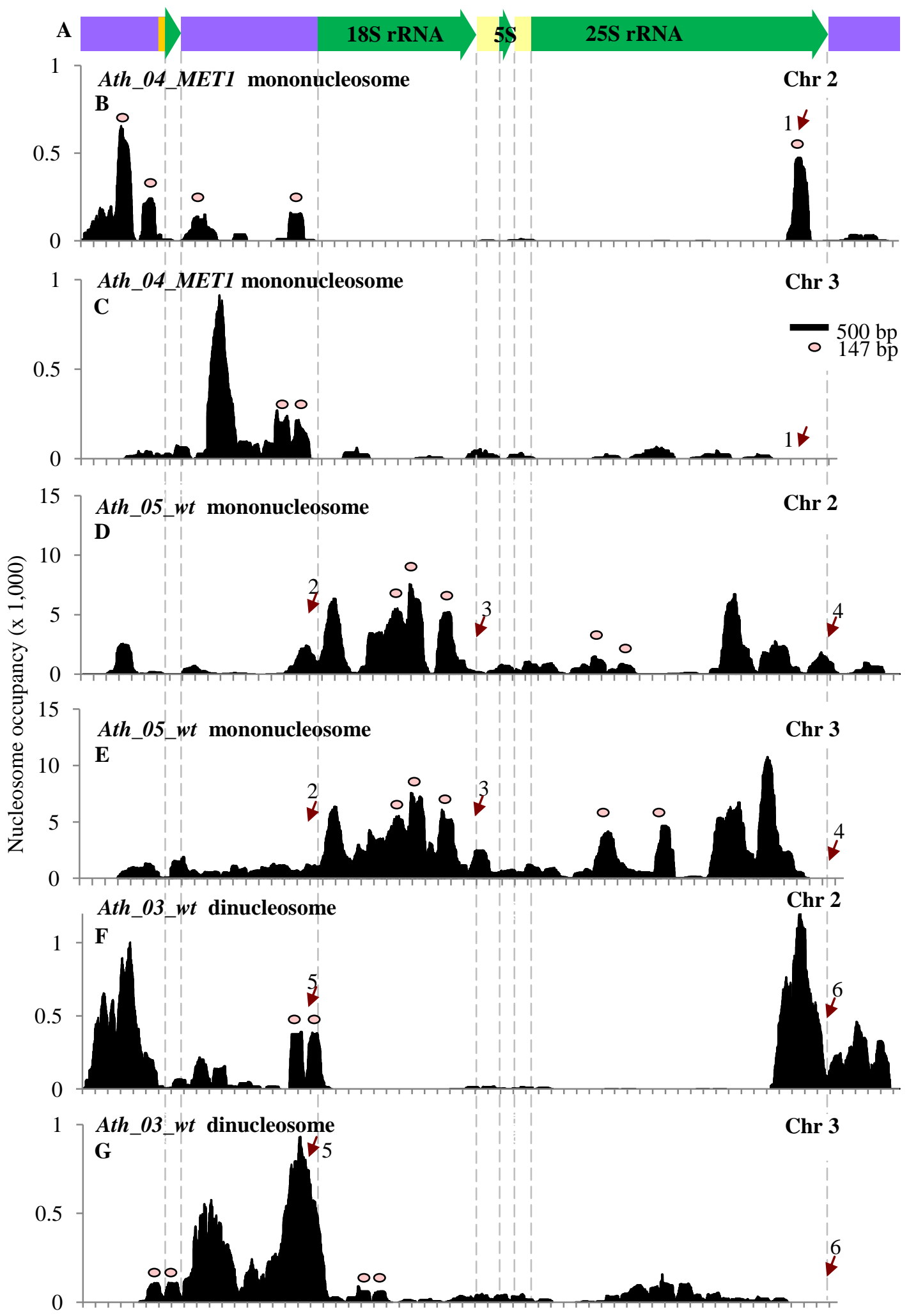

Figure 5.13 Alignment of (A) rRNA genes on chromosomes 2 and 3 and nucleosome occupancy of datasets Ath_04_MET1 (B and C), Ath_05_wt,(D and E), and Ath_03_wt (F and G). The relative positions of the rRNA genes, ITS and IGE are shown in track A. For size comparisons, ellipses represent mononucleosome size (147 bp) and $x$ axis tick marks are spaced at 147 intervals. Arrows 1, 4 and 6 indicate differences in occupancy at 3' end of 25S rRNA gene between chromosomes 2 and 3, and arrows 2, 3 and 5 indicate differences in occupancy around the $18 \mathrm{~S}$ rRNA gene. 
Nucleosome distribution and patterns of occupancy In addition to differences in nucleosome positioning and occupancy over rRNA regions between chromosomes 2 and 3, differences are also observed between the datasets Ath_03_wt, Ath_04_MET1 and Ath_05_wt over the rRNA region within Chr 2 and $\mathrm{Chr} 3$. To enable ready comparisons between datasets over the rRNA region on the same chromosome, the nucleosome occupancies of datasets Ath_03_wt, Ath_04_MET1 and Ath_05_wt were re-plotted against either Chr 2 or Chr 3 (Figures 5.14 and 5.15 , respectively).

The most apparent differences between the datasets on Chr 2 are within the occupancy of nucleosomes from the dataset Ath_05_wt compared to the other two datasets. The observed nucleosome occupancy around the 18S rRNA gene was much higher in the Ath_05_wt dataset, than the other two datasets. The occupancy of the 5S and 5' end of the 25S rRNA genes is also relatively higher in the Ath_05_wt dataset than the Ath_03_wt or Ath_04_MET1 datasets. The occupancy over the three rRNA gene regions is either relatively low or absent in the Ath_03_wt and Ath_04_MET1 datasets, giving the impression of nucleosome-free regions over these genes. In addition, higher peaks in nucleosome occupancy at the $3^{\prime}$ end of the $25 \mathrm{~S}$ rRNA gene in the Ath_03_wt and Ath_O4_MET1 datasets appear to correspond to a low occupancy region in the Ath_05_wt dataset.

A similar pattern of nucleosome occupancy within the $18 \mathrm{~S}$ and $25 \mathrm{~S}$ rRNA is also observed in Chr 3 (Figure 5.15). The Ath_05_wt dataset shows well-ordered high occupancy compared with the much lower occupancy for Ath_03_wt and Ath_04_MET1 datasets over the same gene. The datasets Ath_03_wt and Ath_04_MET1 appear to show more similarities than either the two mononucleosome datasets (Ath_05_wt and Ath_04_MET1) or the two wild-type datasets (Ath_03_wt and Ath_05_wt). 


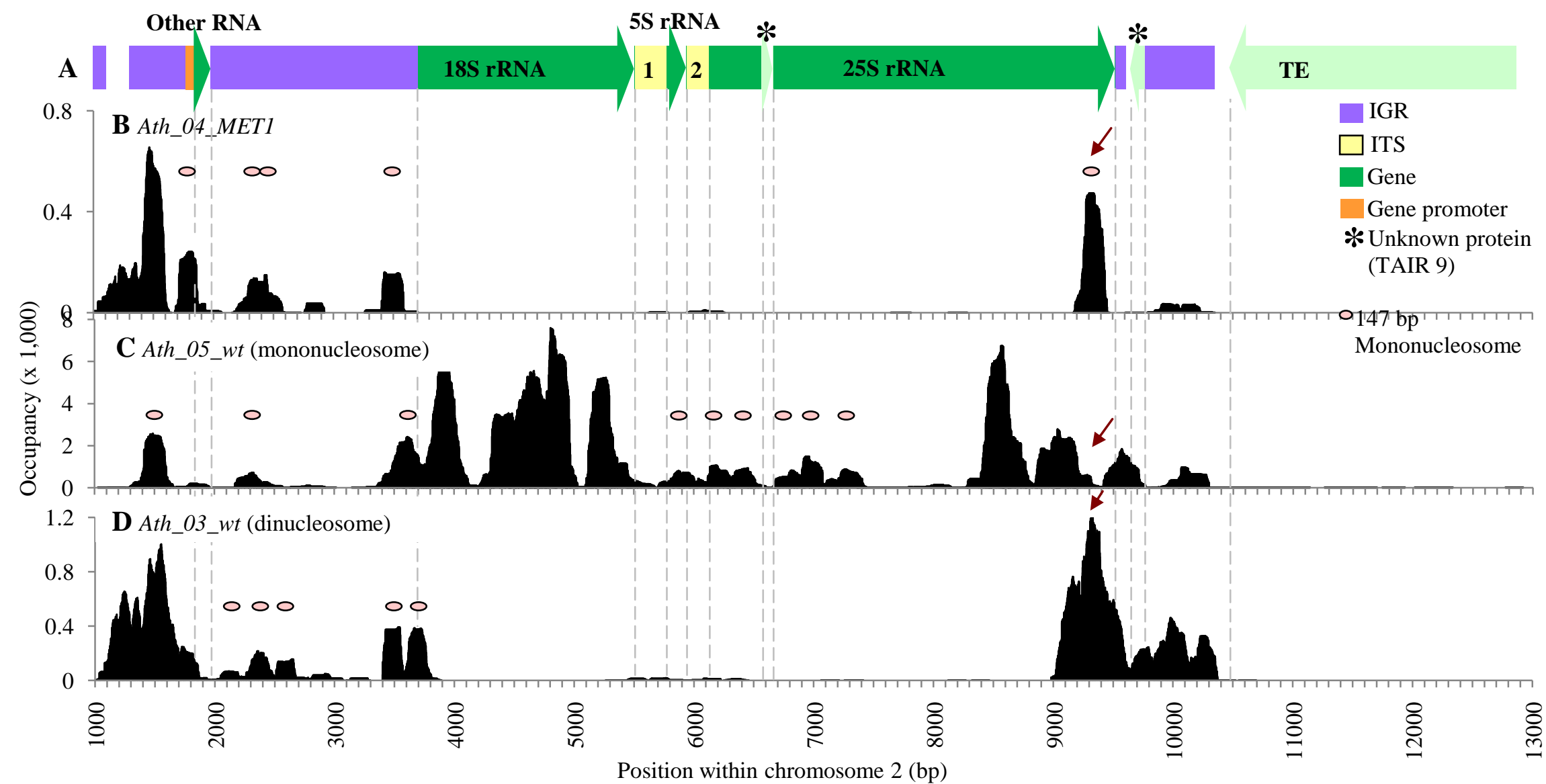

Figure 5.14 Nucleosome occupancy of the rRNA regions on chromosome 2 for datasets B: Ath_04_MET1, C: Ath 05_wt and D: Ath 03 wt. The positions of the rRNA genes, ITS and IGR are indicated in track A. For size comparisons, ellipses represent mononucleosome size (147 bp). The arrows indicate similarities in occupancy between Ath_O0_wt and Ath_04_MET1 dataset not observed in the Ath_05_wt dataset. 


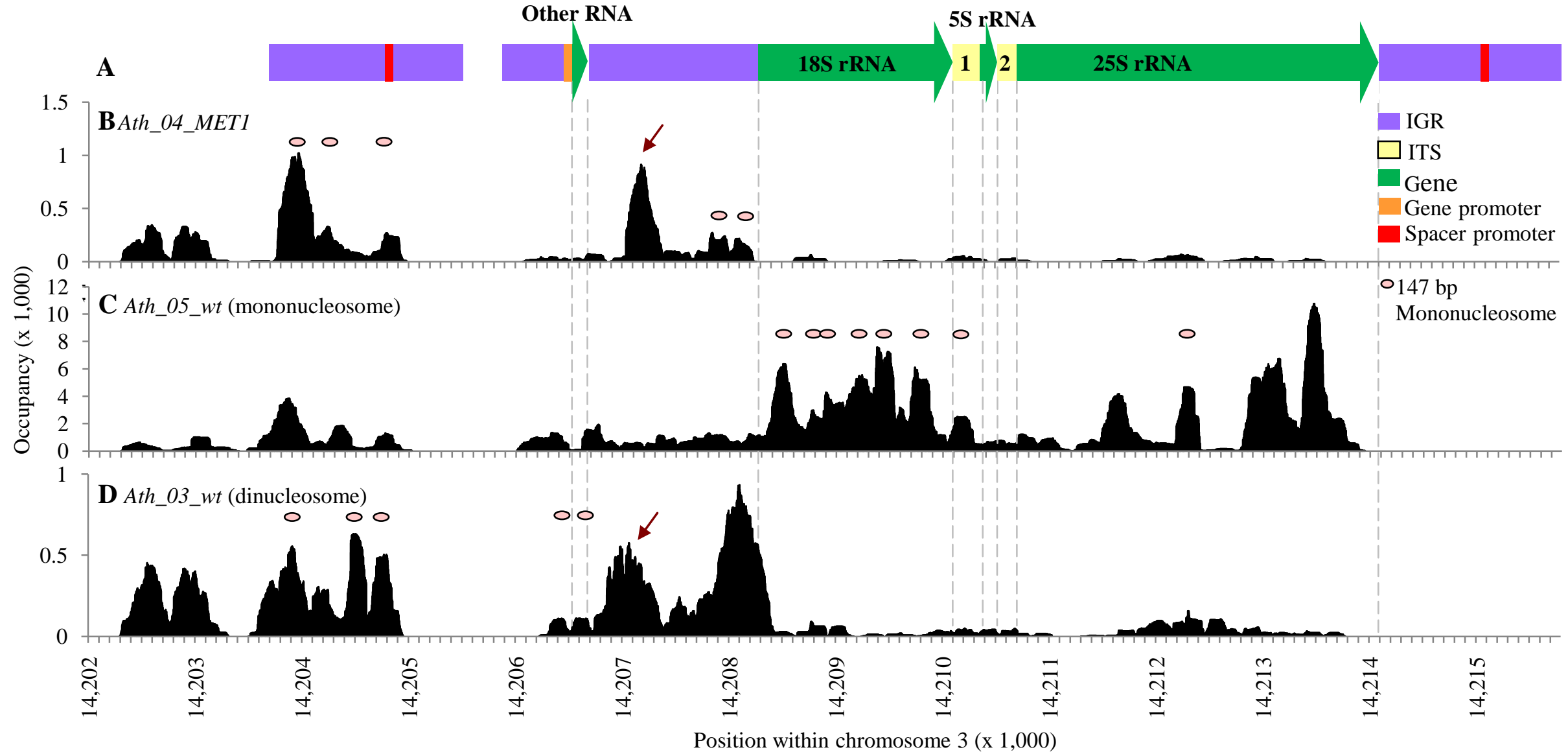

Figure 5.15 Nucleosome occupancy of the rRNA regions on chromosome 3 for datasets B: Ath_04_MET1, C: Ath_05_wt and D: Ath_03_wt. The positions of the rRNA genes, ITS and IGR are indicated in track A. For size comparisons, ellipses represent mononucleosome size (147 bp). The arrows indicate similarities in occupancy between Ath_03_wt and Ath_04_MET1 dataset not observed in the Ath_05_wt dataset. 


\subsection{Discussion}

\subsubsection{Genomic trends of nucleosome distribution}

Nucleosomes were found to be distribution throughout the Arabidopsis genome, in a non-uniform manner. and with particular regions of high occupancy. Of particular interest was the positioning within the rRNA regions annotated on chromosomes 2 and 3. These observations are consistent with previous observations in yeast (Yuan et al., 2005) and C. elegans chromatin (Johnson et al., 2007). It is not surprising that peaks are observed around rRNA, as these regions consist of highly repeated sequences, some of which may not be represented by the correct copy number within the current Arabidopsis annotation. In addition, transposable elements are known to be highly methylated (epigenetically regulated), and also contain repetitive sequences (Lippman et al., 2004). It should be noted that the datasets Ath_03_wt, Ath_04_MET1 and Ath_05_wt were filtered for multiple hits within the genome (for the $32 \mathrm{bp}$ and $76 \mathrm{bp}$ tags) prior to these analyses.

Genic regions were found to be occupied more often than intergenic regions in wildtype Arabidopsis chromatin as can be seen within the two examples of nucleosome occupancy from chromosome 1 (Figures 5.7 and 5.8). This observation of nucleosome occupancy is also consistent with previous observations in yeast (Lee et al., 2007). Nucleosomes were found to be present in $87 \%$ of the transcribed sequences in yeast, in contrast to $53 \%$ of the intergenic sequences (Lee et al., 2007). This could be due to the positioning of nucleosomes, with the intergenic regions being less well defined than positioning over specific transcribed sequences. This in turn may lead to conserved nucleosome positions within the transcribed regions being sampled more often than those within intergenic regions. Alternatively, the chromatin structure (influenced by epigenetic marks) may be different within the transcribed region compared to the intergenic, which may render the chromatin either more resistant or more susceptible to digestion by MNase. The differences observed within this study between the wild-type mononucleosome and dinucleosome datasets, particularly within the rRNA region, certainly seem to support the hypothesis that different classes of nucleosome exist, which also appear to have different susceptibilities to enzymatic digestion. 
Previous studies in yeast have demonstrated both de-localised and well-defined nucleosome positions (Yuan et al., 2005). The de-localised nucleosome positions were associated more often with highly transcribed genes, and as a result were attributed to the disassembling and re-assembling of nucleosomes during the passage of RNA polymerase II through the coding region. In the current project for Arabidopsis, regions of high occupancy with a wide spread, indicating de-localised (or 'fuzzy') positioning were observed here in Arabidopsis. In addition, nucleosome peaks of high occupancy with narrow spread, indicating well-ordered positioning, were also observed. Within the two examples of nucleosome occupancy on Arabidopsis chromosome 1, both the de-localised and well-ordered classes of nucleosomes are observed. However, the de-localised nucleosome positions appear mostly to be confined to the transcriptional units in both Ath_03_wt and Ath_05_wt datasets, which could be due to the passage of Pol II within these genes (Schwabish and Strul, 2004). Well-defined nucleosome positions are observed both within the transcriptional units and within the intergenic regions.

The Ath_04_MET1 dataset appeared to have more instances of de-localised nucleosome positions within the intergenic regions, possibly associated with the loss of ${ }^{5 \mathrm{~m}} \mathrm{CG}$ epigenetic marks. Furthermore, these tend to be located in a region with low occupancy or well-defined nucleosome positions in the corresponding locations in the wild-type datasets. This suggests a de-regulation of positioned nucleosomes in the anti-sense MET1 within genic regions, resulting in higher occupancy in the intergenic regions.

\subsubsection{Nucleosome occupancy in 5' region of PHERES1 and PHERES2}

Differences were observed in the positioning of nucleosomes at 5' and 3 ' ends of genes between the wild-type Ath_03_wt and Ath_05_wt datasets, and also in the Ath_04_MET1 dataset within the examples shown for chromosome 1. The PHERES1 and PHERES2 genes display particularly interesting behaviour with regard to nucleosome occupancy. In wild-type plants, PHERES1 is imprinted on the maternal allele in the developing embryo following fertilisation. Silencing of this allele is brought about by methylation of H3K27 by FIS, a polycomb-group complex of proteins comprising of MEDEA, FIE and FIS2 (Köhler et al, 2006). The paternal 
Nucleosome distribution and patterns of occupancy PHERES1 allele is silenced following fertilisation, by DNA methylation. In antisense MET1, the paternal PHERES1 allele is expressed in the embryo and endosperm, whereas the maternal allele remains repressed (Köhler and Makarevich, 2006). The wild-type datasets Ath_03_wt and Ath_05_wt show nucleosome occupancy at the TSS and within the 5' region of PHERES1. The Ath_04_MET1 dataset shows a loss of occupancy around the TSS of PHERES1. The PHERES2 gene is thought to have a similar temporal pattern of transcription to PHERESI (Villar et al, 2009). The Ath_04_MET1 dataset showed increased occupancy of PHERES2 between the TSS and 300 bp upstream of the TSS, which was not observed in the Ath_03_wt and Ath_05_wt datasets. These observations suggest that differences may exist in transcriptional regulation within the leaves of Arabidopsis anti-sense MET1 as nucleosome occupancy within the 5' region upstream of the TSS is thought to be important for transcriptional regulation (Yuan et al., 2005). To test this hypothesis, the transcript levels of PHERES1 and PHERES2 would need to be measured in the same material from which the chromatin was extracted.

\section{$\underline{\text { 5.4.3 Nucleosome occupancy within exons and introns }}$}

The exon/intron ratio of nucleosome occupancy within chromosome 1 was found to be altered in the Ath_04_MET1 datasets compared with the wild-type. Calculations of the ratio of exons and introns for various annotation classes revealed that for protein-coding regions, nucleosome occupancy is skewed towards exonic DNA in protein coding, 5' UTR and 3' UTR regions. Occupancy has also been shown to be skewed in the yeast genome (Lee et al., 2007). The Ath_04_MET1 dataset ratio was nearer to that of the exon/intron ratio of the whole chromosome. This suggests that nucleosomes are positioned more often in the exons than introns in wild-type Arabidopsis, and that exon-specific positioning may be compromised in the Arabidopsis anti-sense MET1. In wild-type Arabidopsis with normal 5'me-C levels, one third of the transcribed genes are methylated within the gene body (Zhang et al., 2006). It may be that the gene body DNA methylation either directly or indirectly guides nucleosome positioning towards exons. Methylation within the gene body occurs almost exclusively in the [CG] context. Gene body methylation has been shown to be higher within in the transcribed regions when compared to the upstream and downstream regions and be skewed towards the 3' end of the transcribed region (Cokus et al., 2008). In addition, the profiles of methylation within the transcribed 
Nucleosome distribution and patterns of occupancy

regions were reported for a number of single, double and triple methylation mutants. This demonstrated a complete loss of [CG] methylation in the met-1 mutant. However, when met-1, drm1 and drm2 are all missing, [CG] methylation within the transcribed region appears to be compensated by CMT3 with $\mathrm{CHG}$ methylation which occurs in a similar distribution profile to the wild-type [CG] profile (Cokus et al., 2008).

\section{$\underline{\text { 5.4.4 Nucleosome occupancy at the transcriptional start site }}$}

Previous studies in yeast have demonstrated a nucleosome-free region of $150 \mathrm{bp}$ in length, approximately 200 bp upstream from annotated genes (Yuan et al., 2005). The nucleosome occupancy of the first $6 \mathrm{Mbp}$ of Arabidopsis Chr 1 was investigated in the Ath_05_wt dataset, and a nucleosome-free region of this size (150 bp) was not observed in this subset. However, the nucleosome occupancy was lower within the upstream (-1000 to $0 \mathrm{bp}$, relative to the TSS) region than in the downstream region. This is consistent with a further study in yeast (Lee et al., 2007) and more recently in Drosophila (Mavrich et al., 2008a). A clear peak in nucleosome occupancy was observed in Arabidopsis around +25 to +70 bp relative to the TSS, with the corresponding peak in the smoothed distribution at $+64 \mathrm{bp}$. As the nucleosome distances were measured from the midpoint, this would place the TSS within the first $10 \mathrm{bp}$ of the nucleosome. This is in contrast to the positioning around the TSS in yeast and Drosophila (Mavrich et al., 2008a). For comparison, the Arabidopsis nucleosome occupancy around the TSS with the smoothed distribution overlaid is aligned with the occupancy for yeast and Drosophila (Figure 5.16).

While the position of the first peak downstream of the TSS (the +1 nucleosome) differs between all three taxa, the position of +2 and +3 peaks in the Arabidopsis distribution appear to coincide with +2 in yeast and +2 in Drosophila, respectively. The position of the -1 nucleosome was found to be conserved in Drosophila and yeast, but does not appear to be conserved in Arabidopsis, with the first main peak being further upstream than in yeast or Drosophila. However, well ordered nucleosome positioning is observed both upstream and downstream of the TSS in all distributions, suggesting that while the position of the occupancy differs between taxa, well-ordered, long-range nucleosome positioning around the TSS is present in all three and is likely to contribute to gene regulation. 


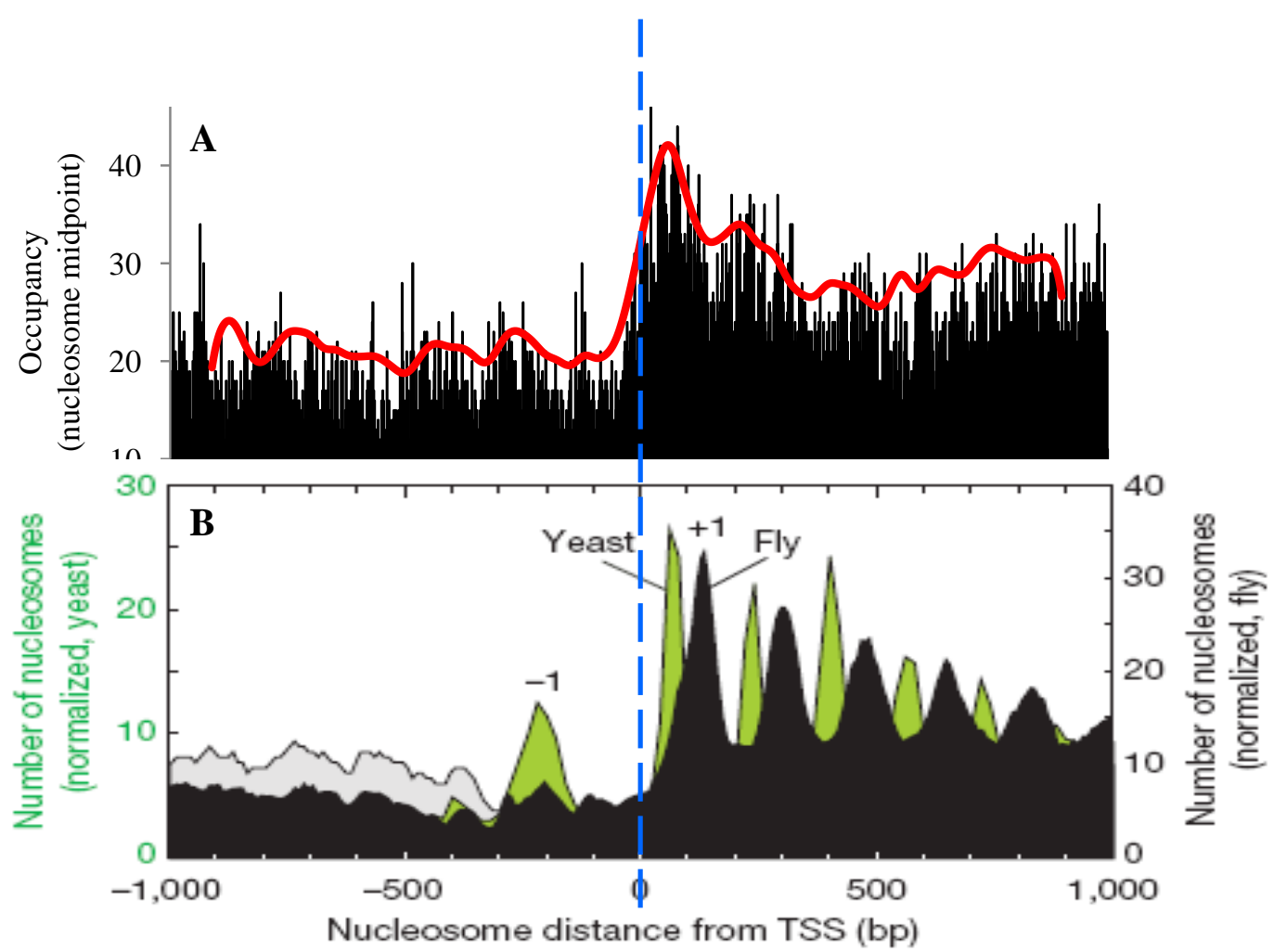

Figure 5.16 The nucleosome occupancy around the TSSs of Arabidopsis, yeast and Drosophila. A: the occupancy around the TSS of the first $6 \mathrm{Mbp}$ in Arabidopsis chromosome 1, with the smoothed distribution overlaid. B: the H2A.Z-containing nucleosome positioning around the TSS of yeast and Drosophila. Image from Mavrich et al., (2008). The blue dashed line indicates the position of the TSS.

A study of the H2A.Z histone variant positions in yeast position the TSS within the first helical turn of the nucleosome (Albert et al., 2007). This is consistent with the peak in occupancy in Arabidopsis between +63 and $+70 \mathrm{bp}$, relative to the TSS.

\subsubsection{Nucleosome occupancy with the Arabidopsis nucleolar organiser regions}

The rRNA genes in the NORs of Arabidopsis display well-conserved non-random positioning. The annotation of single copies of these genes account for $\sim 700-900$ copies within each NOR (assuming the single repeat on Chr 3 represents the NOR on Chr 4). High occupancy around the NOR was also observed in C. elegans chromatin (Johnson et al., 2007). Differences between the two annotated NORs are observed in all datasets Ath_03_wt, Ath_04_MET1 and Ath_05_wt. These differences may represent variation in regulation over the two NORs, particularly with the peaks observed in the Ath_05_wt dataset around the 18S rRNA gene. 
Nucleosome distribution and patterns of occupancy A peak at 100-150 bp upstream of the 5' edge was observed on Chr 2 but not on Chr 3, while a peak at the 3' end was observed on Chr 3 but not on Chr 2. In addition, the Ath_03_wt dataset has a well-defined dinucleosome at the 5' end of the 18S rRNA gene on Chr 2, and a de-localised peak over the same region on $\mathrm{Chr} 3$. The de-localised (or fuzzy) nucleosome positions are thought to correspond with highly expressed genes (Yuan et al., 2005). Whilst a complete NOR is not thought to be silenced or active (Preuss and Pickaard, 2007), these results suggest that one NOR may be relatively more active and the other may be more silenced. The level of occupancy within the annotated rRNA regions is 1,000 larger than other regions of the genome, which is consistent with conserved positioning across the 700-900 copies of the rRNA region in each NOR.

The differences observed over the rRNA regions between the Ath_03_wt and Ath_05_wt datasets were unexpected. The region corresponding to the position of the genes on both chromosomes appear to be occupied by mononucleosomes, while dinucleosomes tended to occupy the IGRs. This could indicate differences in susceptibility to enzymatic digestion resulting in different classes of nucleosomes being present in the bands of a nucleosome ladder. The two wild-type datasets Ath_03_wt and Ath_05_wt were produced from the same material, in the same chromatin digest and cut from the same gel. The known difference between the samples was the size of the fragment (and that the populations of fragments were sequenced on separate occasions). Had the Ath_05_wt dataset not been sequenced, it might have been concluded that the 18S rRNA gene was a nucleosome-free region, as there was far less occupancy over the gene in this dataset than in the other two datasets. This is an important issue as many conclusions made regarding the positioning of nucleosomes relative to annotated positions are based on one type of molecule (many mononucleosomes). It may be the case that the fragment size resulting from chromatin digestion gives insight into the high-order chromatin structure.

\subsubsection{Summary}

The distribution of nucleosomes within chromosome 1 was relatively even, with occasional peaks observed around transposable elements, centromeres and rRNA regions. Genic regions were found to be occupied more often than intergenic 
Nucleosome distribution and patterns of occupancy regions, which is consistent with other studies. However, for the Ath_04_MET1 dataset, the ratio of genic to intergenic regions occupied by nucleosomes is closer to the genomic ratio for genic to intergenic regions. This indicates a more even distribution of nucleosome positions. Differences in the nucleosome occupancy around the 5' ends of the PHERES genes are observed between wild-type and antisense MET1 chromatin, suggesting alternative regulation of these genes in the antisense MET1. Nucleosomes exhibit specific patterns of occupancy within introns and exons, and around the TSS. Differences in nucleosome occupancy around the TSS were observed between Arabidopsis and other taxa, with well-ordered positioning a feature in all taxa. Within the NORs, subtle differences are observed in the nucleosome occupancy between chromosomes in each dataset, indicating differences in regulation of the rRNA genes. However, more surprising were the differences observed between nucleosome classes, suggesting that chromatin regions have different susceptibilities to enzymatic digestion. 
Chapter 6

Effect of DNA methylation on nucleosome position 


\subsection{Introduction}

DNA methylation occurs at [CG] nucleotides in mammals, and [CG], [CNG] and [CNN] in plants. DNA methylation through MET1 occurs in the context of [CG]. The DNA pericentromeric regions, DNA repeats and transposable elements are all highly methylated in Arabidopsis in all sequence contexts (Cokus et al., 2008). In addition, relatively high levels of DNA methylation have been found within the gene body of over one third of expressed genes in Arabidopsis, with 5\% methylated within the promoter (Cokus et al., 2008). Furthermore, genes with methylation in the gene body tend to be constitutively expressed, while genes with promoter methylation tend to be developmentally controlled, indicating that transcriptional control by DNA methylation is position-dependent (Zhang et al., 2006). Arabidopsis DNA methyltransferase anti-sense MET1 plants have developmental abnormalities, including decreased size, decreased apical dominance, abnormal leaf morphology, abnormal flower morphology and decreased fertility (Finnegan et al., 1996). However, the effect of loss of [CG] methylation from transposable elements is thought to be minimal as MET1 and CMT3 have been shown to act redundantly to repress the mobility of CACTA elements (Kato et al., 2003).

Experiments involving whole-genome bisulphite sequencing have revealed patterns of DNA methylation in Arabidopsis in detail. There appear to be different types of methylation within the different functional and structural components of Arabidopsis, with complex interactions between methyltransferases acting to control levels of DNA methylation. The methylation of pericentromeric regions, DNA repeats and transposable elements occurs at $[\mathrm{CG}],[\mathrm{CHG}]$ and $[\mathrm{CHH}]$ di/trinucleotides, while gene body methylation occurs at [CG] dinucleotides (Cokus et al., 2008). In addition, $55 \%$ of the methylation in DNA from floral tissue was found to be at [CG] dinucleotides (Lister et al., 2008). In the met-1 mutant (met1-3) the level of [CG] methylation is $>1 \%$ of that in the wild-type, although [CHG] methylation is increased, and is found within the gene bodies of more than 2,000 genes (Lister et al., 2008). This study also compared the sites of DNA methylation with the snRNAome and found snRNAs associated with one third of the sites of DNA methylation and down-regulation of snRNA transcription in the met- 1 mutant. 
Effect of DNA methylation on nucleosome position There have been few studies of the effect of DNA methylation on nucleosome positioning (Pennings et al., 2005). Nucleosomes containing $\mathrm{H} 1$ have been shown to form on methylated DNA in mouse (Ball et al., 1983). However, DNA methylation has been shown to alter the position of a nucleosome in the chicken $\beta^{A}$-globin promoter (Davey et al., 2004). The $\beta^{\mathrm{A}}$-globin promoter contains a $[\mathrm{CpG}]_{3}$ element which occupies a position approximately 1.5 helical turns from the dyad axis in vitro, which was disrupted when the DNA was methylated. However, DNA methylation was not shown to alter the nucleosome positioning over the imprinting control region of the mouse Igf2r gene (Davey and Allan, 2003). Whole-genome analysis of the histone variant H2A.Z revealed an antagonistic relationship between DNA methylation and H2A.Z-containing nucleosomes (Zilberman et al., 2008). H2A.Z containing nucleosomes were excluded from highly methylated regions such as the pericentromeric region. In addition, mutation of the complex responsible for deposition of $\mathrm{H} 2 \mathrm{~A} . \mathrm{Z}$ resulted in genome-wide hypermethylation. This suggests that wild-type levels of H2A.Z-containing nucleosomes protect euchromatic DNA from DNA methylation.

With this in mind, the aim of this chapter was to determine if patterns of nucleosome occupancy over the Arabidopsis rRNA regions correlate with the pattern of DNA methylation in the same region, using publicly-available DNA methylation data (Lister et al., 2008). 


\subsection{Methods}

To enable comparisons between the nucleosome occupancy and DNA methylation data, the positions of DNA methylation (Lister et al., 2008) were added to the annotation files as previously described (Chapter 2, section 2.2.vi), by assignment of the methylation score to each position along the Arabidopsis genome. These datasets were generated by Graham King, Rothamsted Research.

\subsection{Results}

In order to investigate the effect of DNA methylation, nucleosome occupancy was compared to previously published ${ }^{5 \mathrm{~m}} \mathrm{C}$ in Arabidopsis wild type the met-1 mutant (Lister et al., 2008). Due to time limitations and tissue differences, comparisons were made over sequences representing NOR regions only.

$\underline{\text { 6.3.1 Nucleosome occupancy and DNA methylation within the Arabidopsis nucleolar }}$ organiser region (NOR).

The Arabidopsis rRNA regions, which are annotated on chromosomes 2 and 3, have been shown in this project to vary in nucleosome linker length distribution and in nucleosome occupancy (Chapter 4, Section 4.3.4. and Chapter 5, Section 5.3.4). In order to test the hypothesis that DNA methylation and nucleosome positioning, the nucleosome occupancy from datasets Ath_03_wt, Ath_05_wt and Ath_04_MET1 over the rRNA regions annotated on Chrs 2 and 3 were plotted (Figures 6.1 and 6.2). The DNA methylation data for wild-type were plotted along with the Ath_03_wt and Ath_05_wt datasets.

There appears to be some distinct patterns of DNA methylation with respect to nucleosome position between Chrs 2 and 3. For the Ath_05_wt dataset, the peaks in DNA methylation appear to coincide with the boundaries of the nucleosome occupancy specifically over the $18 \mathrm{~S}$ and $25 \mathrm{~S}$ rRNA genes on Chr 2 (indicated by black arrows Figure 6.1). In addition, the region immediately upstream of the $5 \mathrm{~S}$ rRNA gene appears to be highly methylated (compared to the $18 \mathrm{~S}$ and $25 \mathrm{~S}$ rRNA genes), indicated by red arrow 1 (Figure 6.1). The nucleosome positioning relative 


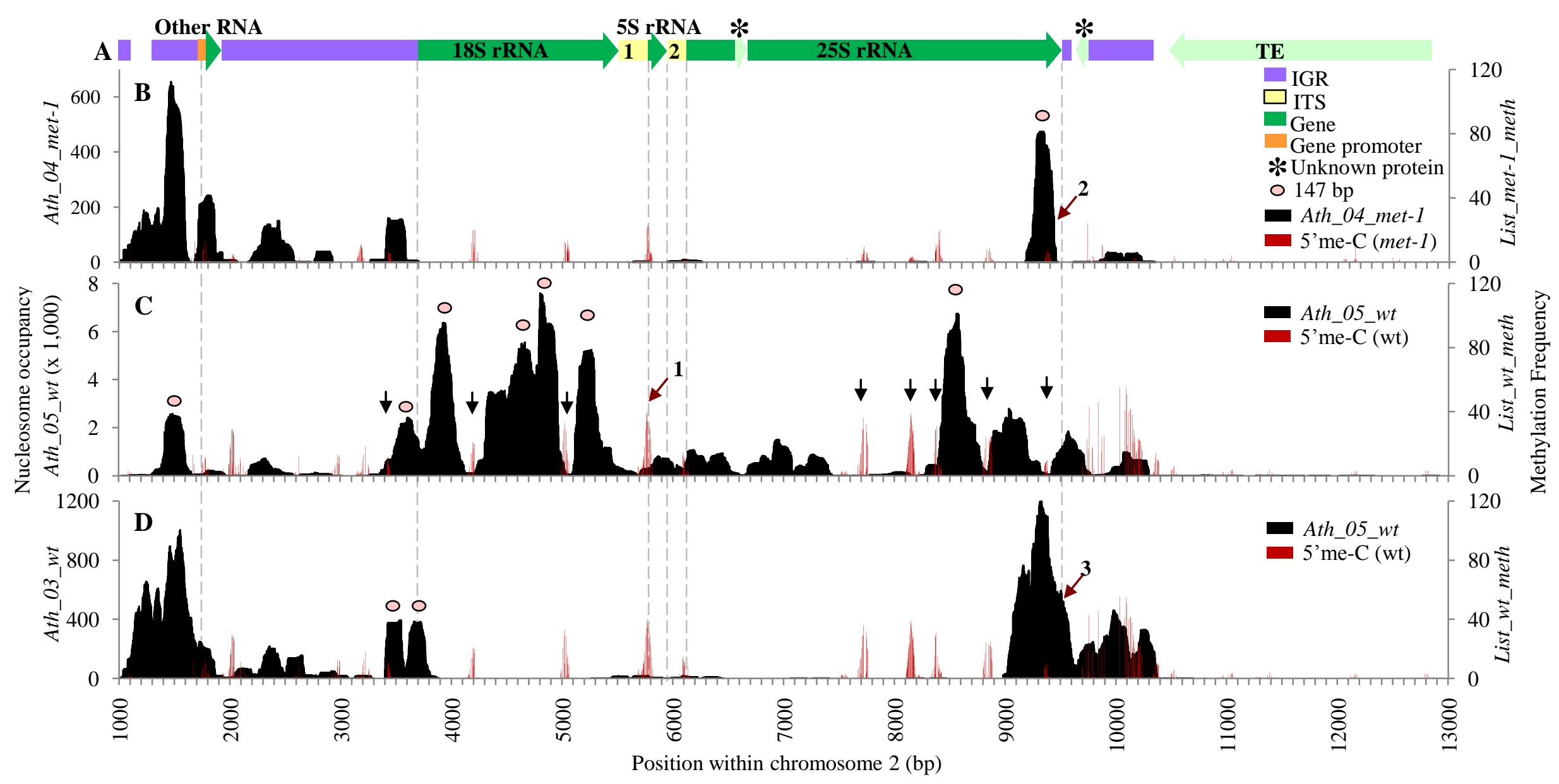

Figure 6.1 Position of 5me-C relative to nucleosome positions over the rRNA region on chromosome 2. A: relative positions of the rRNA genes, B: nucleosome dataset Ath 04 met-1 and ${ }^{5 \mathrm{me}} \mathrm{C}$ levels in met-1 (Lister et al., 2008), C: Ath 05 wt datasets and position of 5me-C in wild-type and D: the nucleosome positions of dataset Ath 03 wt and wild-type ${ }^{5 \mathrm{me}} \mathrm{C}$. Black arrows indicate DNA methylation between nucleosome peaks. Red arrow (1) indicates high methylation at the 5 , end of the 5S rRNA gene, red $\underset{\sigma}{\sigma}$ arrows 2 and 3 indicate methylation within the nucleosome peaks in datasets Ath_04_met-1 and Ath_03_wt, respectively. 


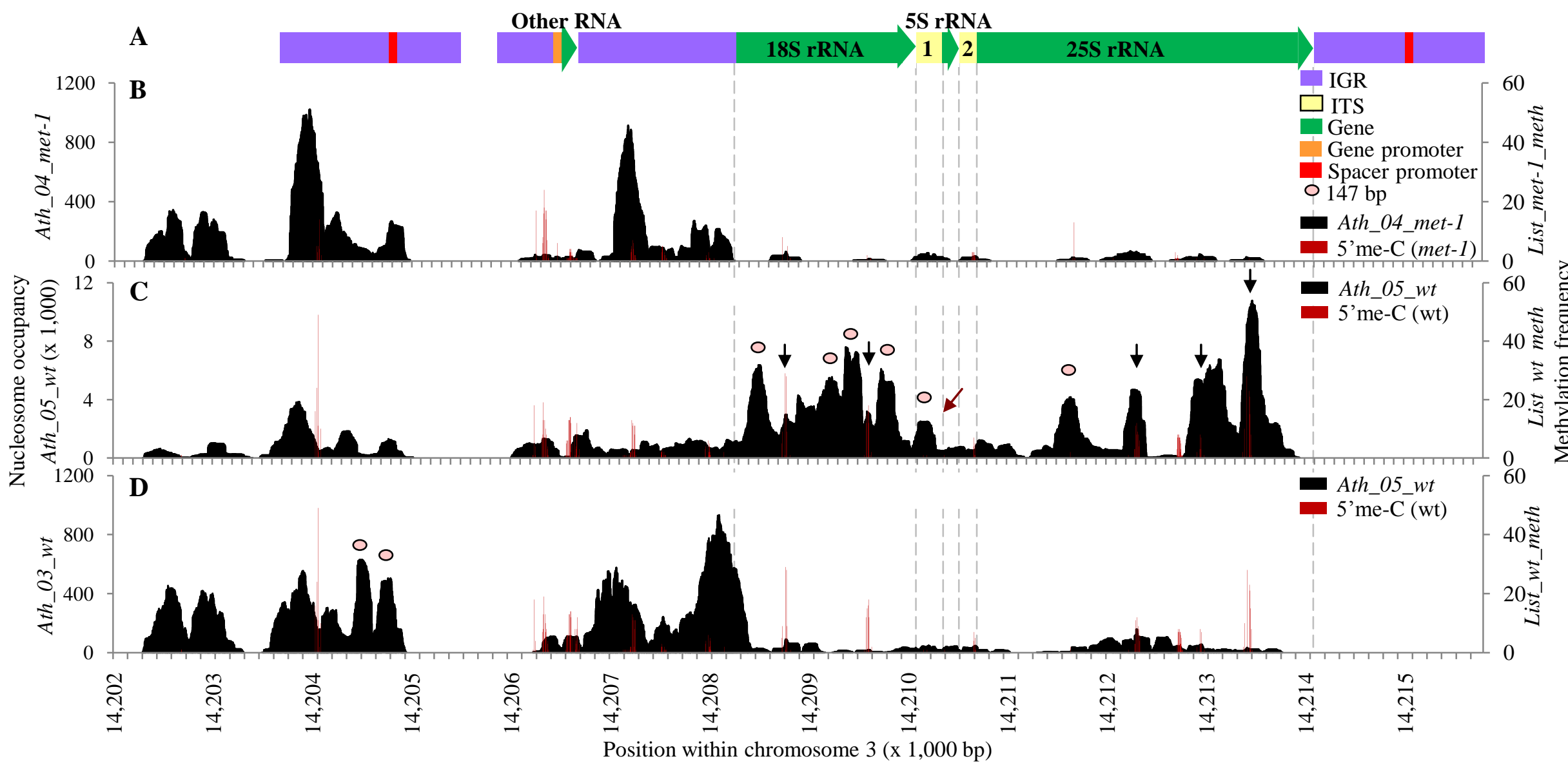

Figure 6.2 Position of $5 \mathrm{me}-\mathrm{C}$ relative to nucleosome positions over the rRNA region on chromosome 3. A: relative positions of the rRNA genes, B: nucleosome dataset Ath 04 met-1 and ${ }^{5 \mathrm{me}} \mathrm{C}$ levels in met-1 (Lister et al., 2008), C: Ath 05 wt datasets and position of 5me-C in wild-type and D: the nucleosome positions of dataset Ath 03 wt and wild-type ${ }^{5 \mathrm{me}} \mathrm{C}$. Black arrows indicate DNA methylation within nucleosome peaks. The red arrow indicates the position of the high methylation observed at the 5 , end of the 5S rRNA gene on Chr 2 but is absent from $\mathrm{Chr} 3$. 
to the position of the DNA methylation appears to be different in the Ath_04_MET1 dataset.

The DNA methylation in the Ath_04_MET1 occurs predominantly at [CNG] sites (anti-sense MET1 CG methylation <20\% of wild-type levels) and is about half of the level of methylation in the wild-type, but appears to be in the same positions. The nucleosome peak adjacent to the 3' boundary of the 25S rRNA gene of datasets Ath_03_wt and Ath_04_MET1 appears to coincide with a peak in the DNA methylation, (as opposed to the edges for Ath_05_wt) and is indicated by red arrows 2 and 3 .

In contrast to $\mathrm{Chr} 2$, for $\mathrm{Chr} 3$, the DNA methylation occurs within the nucleosome peaks of the Ath_05_wt dataset (indicated by black arrows, Figure 6.2). Closer inspection shows that the methylation marks appear to be in similar positions on Chrs 2 and 3, with respect to the positions of the annotated rRNA genes. The levels of DNA methylation on Chr 3 appears to be about half of that on Chr 2. In addition, the DNA methylation observed at the 5' edge of the 5S rRNA gene on Chr 2 appears to be absent from the corresponding position on $\mathrm{Chr} 3$. 


\subsection{Discussion}

The rRNA genes annotated on chromosomes 2 and 3 show differences in the nucleosome positioning relative to the position of the ${ }^{5 \mathrm{~m}} \mathrm{C}$. The differences in position of the DNA methylation in relation to the nucleosome occupancy have not previously been demonstrated. The sequence of the coding regions of the rRNA genes is highly conserved in Arabidopsis (Riddle and Richards, 2002), however, variation in the epigenetic modifications have been shown (Woo and Richards, 2008).

The difference in methylation around the 5S rRNA gene between chromosome 2 and 3 was particularly interesting. The 5' and immediate upstream region of the 5S rRNA gene on Chr 2 appeared to be heavily methylated (compared to $\mathrm{Chr} 3$ ). There also appears to be a denser region of DNA methylation near the gene promoter on $\mathrm{Chr} 2$, whereas the DNA methylation is immediately upstream and downstream of the gene promoter on $\mathrm{Chr} 3$. Previous studies have suggested that promoter-methylation occurs in genes which are developmentally regulated or transcriptionally silent (Zhang et al., 2006). This suggests that there may be more transcriptionally active copies of the rRNA genes in the region annotated on Chr 3 than Chr 2.

The differences observed in nucleosome position between Chrs 2 and 3 may reflect differences in histone modifications between the two regions. Studies of nucleolar dominance in Arabidopsis allotetraploids have revealed that the rRNA genes from one parent are transcriptionally silenced, and that the silencing is reversed by treatment with DNA methylation inhibitors of histone deacytalase inhibitors (Earley et al., 2006). This suggests that the silencing of rRNA genes in genetic hybrids has an epigenetic basis. It is hypothesised that similar mechanisms control rRNA gene dosage in Arabidopsis diploids (Lawrence et al., 2004). It would be useful to compare these data with the positions of various histone modifications such as H3K9me ${ }^{3}$ (Zhang et al., 2007), and H2A.Z-containing nucleosomes (Zilberman et al., 2008), associated with transcriptional repression and activation, respectively. 
Effect of DNA methylation on nucleosome position

\section{$\underline{\text { 6.4.1 Summary }}$}

In summary, nucleosome occupancy over rRNA sequences representative of regions from two chromosomes was compared to the DNA methylation at the same sites obtained from previously published data. This revealed differences between the rRNA regions with respect to nucleosome positioning and DNA methylation not previously reported. DNA methylation was found to occur between peaks in nucleosome occupancy on Chr 2 and within peaks on Chr 3. It is likely that the differences represent differences in histone modifications over the two regions. 
Chapter 7

General Discussion 


\section{General discussion}

This thesis has presented an analysis of nucleosome positioning data for the Arabidopsis genome gathered by sequencing of nucleosome DNA fragments, using three different sequencing technologies: traditional Sanger sequencing, 454 FLX technology and Solexa technology. The datasets created by the 454 and Solexa sequencing technologies enable genome-wide study of nucleosome positioning preferences across the Arabidopsis genome and are comparable to the studies of nucleosome positioning in other taxa including yeast (Lee et al., 2007), C. elegans (Johnson et al., 2006), Drosophila (Mavrich et al., 2008a) and human (Schones et al., 2008).

\section{$\underline{7.1 \text { Nucleosome positioning in Arabidopsis }}$}

It has been demonstrated in Chapter 3 that Arabidopsis nucleosome core sequences display similar characteristics to those isolated from other taxa, including chicken and yeast. In particular, the [AA/TT] distribution observed within the $147 \mathrm{bp}$ of Arabidopsis nucleosome DNA fragments is a general characteristic of nucleosome sequences (Ioshikhes et al., 1996; Satchwell et al., 1986), although the base preference has been shown to vary between taxa (Kogan et al., 2006). The presence of a nucleosome dinucleotide preference within Arabidopsis nucleosome DNA fragments suggests that, for at least a subset within the Arabidopsis genome, nucleosomes are rotationally positioned (or positioned with respect to the DNA sequence structure/curvature). Previous studies have suggested that nucleosomes tend to be rotationally positioned at the promoter of transcriptionally silenced genes and translationally positioned at transcriptionally active genes (Mahesh et al., 2005; Chen and Yang, 2001). It is likely that there are contributions to the rotational positioning of nucleosomes other than the well-characterised [AA/TT] preference, and periodicities were detected in all of the complementary base combinations.

Evidence presented in Chapter 4 demonstrated a mean linker length for Arabidopsis which is similar to those described for other taxa (Yuan et al,, 2005; Kato et al., 2003; and Satchwell and Travers, 1989). However, it is perhaps more useful to consider the range of linker lengths, as length distributions associated with smaller 
General discussion

datasets are non-normally distributed. While it has been reported that different cell types have different linker lengths (Lohr et al., 1977), it is likely that linker lengths also vary significantly between adjacent regions. It may be that linker lengths only appear uniform in the ordered arrays found in heterochromatin (Yang et al., 2006), or in specific contexts of exons, dependent upon the level of transcriptional activation. A periodicity detected within the linker length distribution of $\sim 10$ bp has been reported previously (Widom, 1992) from sample sizes of 1,001 and 185, respectively. Although this was not detected in the Arabidopsis datasets, a shorter period of $\sim 7-8 \mathrm{bp}$ was found. Following this discovery, the periodicities of the available datasets from other taxa were investigated. This revealed that the reported periodicity of $10 \mathrm{bp}$ was only found in the chicken dataset (which also happens to be the smallest dataset), which may be skewed towards repetitive sequences. However, $\sim 10 \mathrm{bp}$ periodicities have been found in the spacing of nucleosome positioning signals within the human genome (Kato et al., 2003). One reason for the observed variations in periodicity may be that the 'default setting' of nucleosome positioning signals are encoded in the DNA sequence and spaced at (multiples of) 10 bp periods, but the deviations from this detected in the Arabidopsis dinucleosome linker length datasets are a reflection of re-positioning at specific locations within the genome by active chromatin remodelling, such as around the TSS or at exon/intron boundaries. In addition, the difference between the mononucleosome and dinucleosome datasets suggests that the dinucleosomes extracted may represent a subset of nucleosomes with a different susceptibility to enzymatic digestion than the mononucleosomes. Therefore the estimation of linker length from dinucleosome subsets, may result in a bias towards inclusion of only a specific class of nucleosomes.

The difference in linker lengths of dinucleosomes aligned to the rRNA regions of chromosomes 2 and 3 was of particular interest. These differences most likely represent differences in the epigenetic landscape between the two regions.

DNA fragments which uniquely map to the rRNA genes on either Chr 2 or $\mathrm{Chr} 3$ were analysed and some differences between the two regions were observed. There were two peaks within the distribution of linker length for fragments of Chr 3: one around $30 \mathrm{bp}$ and one around $42 \mathrm{bp}$ while the rRNA region on Chr 2 has a peak around $67 \mathrm{bp}$. These findings suggest that the rRNA regions annotated on Chrs 2 
General discussion

and 3 have different predominant chromatin structures, possibly relating to the transcriptional activity of these regions. Analysis of the histone modifications revealed that a fraction of the Arabidopsis rRNA genes are associated with the euchromatic mark H3K4me3 (Lawrence et al., 2004). Furthermore, it was found that a fraction of the rRNA genes associated with heterochromatic $\mathrm{H} 3 \mathrm{~K} 9 \mathrm{me} 2$, suggesting that some Arabidopsis rRNA genes copies are switched on and some are off. It is possible that the structure observed in this study over the rRNA regions may give some clues as to the proportions of each NOR which are active. Since the distributions overlap, it is likely that a dominant structure forms on each NOR. The lack of precision in current available sequence datasets for NORs in Arabidopsis currently hinders detailed analysis. However, it may be possible to test the relative abundance of transcripts with SNPs which correspond to either Chr 2 or 3 as there are likely to be variety of SNPs within the repeated regions. Experiments with Arabidopsis allotetraploids demonstrated that the rRNA genes inherited from $A$. thaliana in the tetraploid A. suecica were silenced and the contribution from $A$. arenosa were transcriptionally active (Chen et al., 1998). However, in synthetic hybrids, under-dominance of the A. thaliana genes can take two generations to establish. It is likely that the transcriptional state, and therefore the chromatin structure, is altered with development.

Approximately half of the Arabidopsis genome was found to be occupied by nucleosomes. This is less than the previously reported $81 \%$ in the 10 times smaller yeast genome (Lee et al., 2007). Nucleosome occupancy was found to be higher in genic regions in wild-type Arabidopsis, which is supported by the finding that nucleosomal DNA has a higher GC content than the genome average (Chapter 3, section 3.3.3.ii). This also agrees with genomic nucleosome positioning data for yeast (Lee at al., 2007). A specific pattern of occupancy in the vicinity of the TSS was demonstrated for Arabidopsis, with a peak (midpoint of the nucleosome) $\sim 60 \mathrm{bp}$ downstream of the TSS. This would position the TSS within the first $10 \mathrm{bp}$ of the nucleosome, which associates with histone H2. A recent study of the interactions between the histone octomer and nucleosomal DNA suggest strong interactions around the dyad, with (weaker) interactions between the histone and DNA observed up to $60 \mathrm{bp}$ from the dyad (Hall et al., 2009). Thus the TSS in Arabidopsis is more likely to be within the region of weakest interaction between DNA and histone 
General discussion

octomer, and may result in Pol II having limited access to the TSS (rather than being excluded from it). Occupancy around the TSS has in the past couple of years been studied in a variety of non-plant eukaryotes, including yeast (Mavrich et al., 2008b; Lee et al., 2007; Albert et al, 2007), Drosophila (Mavrich et al, 2008a) and human (Schones et al., 2008). Interestingly, these studies revealed taxa-specific differences in the organisation of nucleosomes in the vicinity of the TSS. Specific patterns of phasing of nucleosomes around the TSS were observed in all species. The TSSs in yeast tend to be buried $\sim 13 \mathrm{bp}$ inside the border of the +1 nucleosome the first nucleosome downstream of the TSS (Mavrich et al., 2008b), whilst in Drosophila, the +1 nucleosome was found to be 180 bp upstream of the TSS, leaving the TSS unoccupied. Nucleosomes in the vicinity of the TSS in humans were found to be dependent on the transcriptional activity of the gene (Schones et al., 2008). Phased nucleosomes were detected both upstream and downstream of the TSS of active genes, while only one nucleosome was detected in the TSS of non-transcribed genes, downstream of the TSS. Furthermore, the position of the 5' end of the +1 nucleosome was found to be $40 \mathrm{bp}$ downstream of the TSS in active genes and $10 \mathrm{bp}$ downstream in inactive genes. The position of the nucleosome boundary is thought to overlap the position of bound Pol II within inactive genes (Schones et al., 2008). Therefore, the positioning around the TSS in Arabidopsis is similar to that of yeast, burying the TSS within the nucleosome, while the positioning of nucleosomes within the Drosophila and human genomes suggest a different mechanism of transcriptional control. It should be noted that the estimates of Arabidopsis positioning around the TSS presented here are based on a subset of the genome (12\% of Chr 1), and consequently the distribution of occupancy was noisy. However, smoothing of the distribution was effective in revealing the trends of nucleosome positioning in the vicinity of the TSS. Extending the analysis of nucleosome occupancy across the entire Arabidopsis genome should negate the need for smoothing of the distribution.

\subsection{Differences in nucleosome position resulting from loss of CG methylation}

The Arabidopsis anti-sense METl line was included in this study to investigate the effect of DNA methylation on nucleosome positioning. There were some systematic differences in the properties of nucleosome positions observed between anti-sense MET1 and the wild-type datasets. One of the most striking differences was the 
General discussion

difference in linker length distributions. In general, the average linker length within the anti-sense MET1 dataset was higher than those of the wild-type datasets. A periodicity of $9.2-9.8 \mathrm{bp}$ was detected within the distribution of anti-sense MET1, which is close to the published value of $\sim 10 \mathrm{bp}$ for a wide range of organisms (Widom, 1992). One of the explanations for the discrepancies in the periodicity observed is that de-regulation of positioning allows the nucleosomes to revert to a DNA sequence-dependent 'default setting', with the DNA sequence becoming a more important positioning factor than in the wild-type that possesses ${ }^{5 \mathrm{~m}} \mathrm{CG}$. This might suggest that a greater proportion of nucleosomes in anti-sense MET1 are rotationally positioned and the sequence periodicity within the nucleosome DNA reflects the sequence $10 \mathrm{bp}$ periodicity found throughout the genome. This might be reflected in a stronger sequence preference within anti-sense MET1 nucleosome DNA fragments; however the strength of the sequence preference was not tested here. Slight variation in dinucleotide frequencies within the linker regions was observed between anti-sense METI and wild-type. This result suggests that, although the same distributions of dinucleotides are observed within the nucleosome DNA, the nucleosomes are (in general) in different positions in the wild type and anti-sense MET1. This could be due to the increased stiffness of the methylated wild-type DNA molecule, compared to the anti-sense MET1, which is brought about by DNA methylation (Virstedt et al., 2004). Thus the primary consequence of DNA methylation marks may be to influence translational setting of nucleosomes. This is likely to occur through modulation of bendability within the minor groove of the DNA, affecting the ability of the DNA to interact efficiently with the histone octamer (Buttinelli et al., 1998). Methylation of the nucleotides at the chicken $\beta$-globin gene has been found to inhibit nucleosome formation (Davey et al., 1997).

The apparent de-regulation of nucleosome positioning in anti-sense METI compared to the wild-type was demonstrated within small sections of Chr 1 . Whilst the nucleosome occupancy appears to be higher within the coding regions compared to the intergenic regions in both anti-sense MET1 and wild-type, the anti-sense MET1 did have more nucleosome occupancy within intergenic regions compared to the wild-type. The analysis of the relative occupancies in the different annotation classes over Chr 1 seems to support this, suggesting that overall nucleosome occupancy within the Arabidopsis anti-sense MET1 genome are more evenly distributed over the 
General discussion

different annotation classes. There are two possible explanations for the apparent deregulation of nucleosome positioning in anti-sense $M E T 1$, (1) the effect of the interaction between DNA methylation within the nucleosome core and the core histones, and (2) the effect of the interaction between methylated DNA at the nucleosome boundary and linker histones. Linker histone $\mathrm{H} 1$ has shown a preference for methylated DNA (McArthur and Thomas, 1996). In addition, mutants with down-regulated linker histone levels exhibit phenotypes of the DNA hypomethylation methylation mutants (Wierzbicki and Jerzmanowski, 2004). Atomic Force Microscopy of chromatin fibres with normal and elevated levels of DNA methylation revealed that hypermethylated fibres are more compact than those with wild-type levels (only in the presence of linker histone (Karymov et al., 2001), further suggesting that wild-type levels of DNA methylation are required for chromatin fibre condensation. If $\mathrm{H} 1$ has a higher affinity for methylated DNA, nucleosomes could be positioned at the nearest site of DNA methylation in the Arabidopsis anti-sense MET1. This may help to explain the de-localisation of nucleosome positions. It is thought that most nucleosomes are associated with a linker histone $(\sim 80 \%)$ and that the association is transient (Lever et al., 2000). The length of time that a linker histone is associated with the nucleosome seems to depend on the post-translational modifications. Acetylated histones had a shorter association with linker histones, which was thought to be due to a higher rate of exchange by chromatin remodelling (Mistelli et al., 2000). The rate of chromatin remodelling could be tested by comparison of nucleosome occupancy at the same loci over different developmental tissues, and compare to the ${ }^{5 \mathrm{~m}} \mathrm{C}$ within the different tissues to determine whether any interaction occurs between the two epigenetic marks.

The level of non- CG DNA methylation has been found to be higher and more extensive in the met-1 mutant than in the wild-type (Cokus et al., 2008). In the wildtype plants, methylation of the genes body occurs exclusively at the CG dinucleotides, whereas non- CG methylation (along with CG methylation) is found in the pericentromeric and centromeric regions, and within transposable elements (Cokus et al., 2008). In addition, DNA methylation is known to affect the modifications of histones within nucleosomes. The complete loss of CG methylation from Arabidopsis has been shown to result in the loss of $\mathrm{H} 3 \mathrm{~K} 9$ methylation from 
within constitutive heterochromatin (Tariq et al., 2003). The H3K4 di and trimethylation marks are associated with euchromatic transcriptionally active regions and tend to be absent from regions of DNA methylation in Arabidopsis. However, H3K4 mono-methylation is highly correlated with the presence of DNA methylation in the transcribed regions of genes (Zhang et al., 2009). In addition, nucleosomes containing the histone variant $\mathrm{H} 2 \mathrm{AZ}$ have been shown to be associated with transcriptionally active promoter regions (Albert et al., 2007; Mavrich et al., 2008; Schones et al., 2008), and are excluded from regions containing DNA methylation (Zilberman et al., 2008). A deficiency of CG methylation in the Arabidopsis antisense MET1 may lead to the proliferation of euchromatic histone marks, resulting in a more relaxed, open chromatin conformation.

\subsection{Higher-order chromatin structure}

One of the questions raised at the beginning of this study was whether it is possible to predict chromatin higher-order structure from knowledge of nucleosome spacing, and if so, whether the higher-order structure can give any clues linking structure and transcriptional control. Early studies of nucleosome positioning and linker length variation postulated that increases in nucleosome repeat length were correlated with increases in transcriptional activity (Ull and Franco, 1986). In contrast, more recent studies of heterochromatin protein ACF suggest that such nucleosomes are spaced 50-60 bp apart in human heterochromatin (Yang et al., 2006). It is possible that heterochromatic, or silenced, regions have a longer linker length and so a more efficient compaction can occur in the presence of linker histone, which would render more of the DNA inaccessible to regulatory proteins. With this in mind, the differences in linker length observed over the rRNA genes in Arabidopsis were intriguing (Chapter 4, Section 4.3.4). Models and measurements of $30 \mathrm{~nm}$ fibres suggest two classes (Robinson et al., 2007; Wong et al., 2007). This would place the linker lengths of Chr 2 NOR within the large class chromatin fibre and the smaller linker length represented by the rRNA genes on Chr 3 within two different classes in the smaller $30 \mathrm{~nm}$ fibre. Although an individual NOR is thought to be both active and inactive, these results may show a bias towards either the active or inactive state (or that more of the rRNA genes represented by chr3 are active and more on Chr 2 are silenced). One way to test this would be to study the positioning of nucleosomes 
General discussion

over the rRNA regions from condensed $30 \mathrm{~nm}$ fibres, a method which has been previously used for the study of solenoid vs zig-zag compaction (Staynov and Proykova, 2008). As active NORs remain decondensed during metaphase it may be possible to distinguish between the two states. It is worth noting that while there are dominant peaks within the distributions of linker length for each region, there is overlap of the distributions between the two regions. This indicates that there may be chromatin structures which are present in both rRNA regions in varying amounts.

In addition, it may be possible to provide other insights into higher-order chromatin structure from the different datasets generated in this study. Mononucleosome and dinucleosome datasets exhibited systematic differences in nucleosome positioning, which may reflect differences in chromatin condensation and so affect target sites for MNase digestion. Thus oligo-nucleosome bands present in MNase-generated nucleosome ladders may show increasing levels of compaction with increase in oligomer size. This phenomenon is worthy of investigation in the future as it may provide information on the hierarchies of nucleosome organisation present within in vivo chromatin.

\subsection{Technological advances}

During the duration of this project, sequencing technologies have progressed significantly. This enabled the various studies to be undertaken, at increasing precision but also with exponentially increasing size of datasets. In fact, analyses that seemed intractable at the beginning of the project, due to costs and starting material requirements became attainable towards the end. Much effort was expended in attempting to acquire nucleosome positioning data from the Arabidopsis tiling arrays, with four experimental (nucleosome) datasets and one control dataset being produced. Following extensive analysis with the tiling array datasets, a nucleosome signal which was uniformly distinct from the control could not be detected, possibly as a result of biases in probe binding efficiencies. However, these datasets were subsequently superseded by the high resolution high-throughput DNA sequence datasets. 


\section{$\underline{7.5 \text { Conclusions and future work }}$}

The large nucleosome positioning datasets generated in this study by highthroughput technologies allowed the elucidation of details which were not possible with the original smaller datasets (Ath_01_wt, $\mathrm{n}=475)$. However, at the time this dataset was generated, it was the first nucleosome positioning dataset generated from plant chromatin, and was larger than the chicken nucleosome positioning datasets (Drew and Travers, 1985). The Ath_05_wt dataset represents an average of $6.4 \mathrm{x}$ coverage of the genome assuming an average linker length of $46 \mathrm{bp}$ based on the average linker length of the two wild-type dinucleosome datasets. Attempts to determine any patterns of dinucleotide distribution within nucleosome sequences did not yield statistically meaningful results with the smaller Ath_O1_wt dataset, and the coverage was insufficient to identify patterns of occupancy around the TSS.

In would be valuable to compare the nucleosome position datasets generated in this study with those associated with other epigenetic marks, for example H2A.Z (Zilberman et al., 2008), H3K9me3 (Zhang et al., 2007), and presence of H1 when this may become available. Such analyses are likely to provide a more comprehensive understanding of the interactions between $5 \mathrm{mC}$ marks, nucleosome rotational and translational positioning and transcriptional control of different gene families.

It is becoming clear that there are many factors involved in nucleosome positioning, and a complex relationship exists between, DNA sequence, DNA methylation histone modifications, and presence/absence of linker histone and that these factors are context dependant. The datasets generated in this project provide a comprehensive overview of the nucleosome landscape across the whole genome. This first study of the nucleosome positions in a plant genome provides a useful starting point for more detailed comparisons of nucleosome positioning within specific genes at different developmental stages, under different environmental conditions, and in response to biotic and abiotic stresses. Many examples of epigenetic-control of transcriptional regulation now exist in Arabidopsis. For example, FLC is one of the most studied epigenetically controlled developmental switches (Saleh et al., 2008; Shindo et al., 2006; Bastow et al., 2004). In a wider context, understanding of the specific processes controlling genes involved in the 
General discussion

response to environmental stimuli, such as drought and salt stress, have practical applications for economically important crops. 
Chapter 8

References 


\section{References}

Albert, I., Mavrich, T.N., Tomsho, L.P., Qi, J., Zanton, S.J., Schuster, S.C., Pugh, B.F. (2007) Translational and rotational settings of H2A.Z nucleosomes across the Saccharomyces cerevisiae genome. Nature 446: 572-576

Alegre, C., Subirana, J.A. (1989) The diameter of chromatin fibres depends on linker length. Chromosoma 98: 77-80

Allan, J., Rau, D.C., Harborne, N., Gould, H. (1984) Higher-order structure in a short repeat length chromatin. The Journal of Cell Biology 98: 1320-1327

An, W., van Holde, K., Zlatanova, J. (1998) Linker histone protection of chromatosomes reconstituted on 5S rDNA from Xenopus borealis: a reinvestigation. Nucleic Acids Research 26: 4042-4046

Anselmi, C., Bocchinfuso, G., De Santis, P., Savino, M., Scipioni, A. (1999) Dual role of DNA intrinsic curvature and flexibility in determining nucleosome stability. Journal of Molecular Biology 286: 1293-1301

Baldi, P., Brunak, S., Chauvin, Y., Krogh, A. (1996) Naturally occurring nucleosome positioning signals in human exons and introns. Journal of Molecular Biology 263: 503-510

Ball, D.J., Gross, D.S., Garrard, W.T. (1983) 5-Methylcytosine is localized in nucleosomes that contain histone H1. Proceedings of the National Academy of Sciences of the USA. 80: 5490-5494

Bastow, R., Mylne, J.S., Lister, C., Lippman, Z., Martienssen, R.A., Dean, C. (2004) Vernalisation requires epigenetic silencing of FLC by histone modification. Nature 427: 164-167

Beckman, J.S. and Trifonov, E.N. (1991) Splice junctions follow a 205-base ladder. Proceedings of the National Academy of Sciences of the USA. 88: 2380-2383

Bernad, R., Sánchez, P., Losada, A. Epigenetic specification of centromeres by CENP-A, Experimental Cell Research. [Published online ahead of print Aug 3 2009] PubMed: PMID 19660450

Boeger, H., Griesenbeck, J., Stratten, J.S., Kornberg, R.D. (2003) Nucleosomes unfold completely at a transcriptionally active promoter. Molecular Cell 11: 15871598

Boeger, H., Griesenbeck, J., Stratten, J.S., Kornberg, R.D. (2004). Removal of promoter nucleosomes by disassembly rather than sliding in vivo. Molecular Cell 14: $667-673$ 
Boeger, H., Bushnell, D.A., Davis, R., Griesenbeck, J., Lorch, Y., Stratten, J.S., Westove, K.D., Kornberg, R.D. (2005) Structural basis of eukaryotic gene transcription. FEBS Letters 579: 899-903

Bolshoy, A., McNamara, P., Harrington, R.E., Trifonov, E.N. (1991) Curved DNA without A-A: experimental estimation of all 16 DNA wedge angles. Proceedings of the National Academy of Sciences of the USA. 88: 2312-2316

Bowler, C., Benevenuto, G., Laflamme, P., Molino, D., Probst, A., Tariq, M., Paszowski, J. (2004) Chromatin techniques for plant cells. The Plant Journal 39: 776-789

Bradnam, K.R., and Korf, I. (2008) Longer first introns are a general property of eukaryotic gene structure. PLoS One 3: e3093

Bustin, M., Catez, F., Lim, J.H. (2005). The dynamics of Histone H1 Function in chromatin. Molecular Cell 17: 617-620

Buttinelli, M., Minnock, A., Panetta, G., Waring, M., Travers A. (1998) The exocyclic groups of DNA modulate the affinity and positioning of the histone octamer. Proceedings of the National Academy of Sciences of the USA. 95: 85448549

Cao, H., Widlund, H.R., Simonsson, T., Kubista, M. (1998) TGGA repeats impair nucleosome formation. Journal of Molecular Biology 281: 253-260

Caperta, A.D., Neves, N., Morais-Cecílio, L., Malhó, R., Viegas, W. (2002) Genome restructuring in rye affects the expression, organization and disposition of homologous rDNA loci. Journal of Cell Science 115: 2839-2846

Chan, S.W., Zilberman, D., Xie, Z., Johansen, L.K., Carrington, J.C., Jacobsen, S.E. (2004) RNA silencing genes control de novo DNA methylation. Science 303: 1336

Chen, Z.J., Comai, L., Pikaard, C.S. (1998) Gene dosage and stochastic effects determine the severity and direction of uniparental ribosomal RNA gene silencing (nucleolar dominance) in Arabidopsis allopolyploids. Proceedings of the National Academy of Sciences of the USA. 95: 14891-14896

Chen, C. and Yang, T.P. (2001) Nucleosomes are translationally positioned on the active allele and rotationally positioned on the inactive allele of the HPRT promoter. Molecular and Cellular Biology 21: 7682-7695

Cheng, X. (1995) Structure and function of DNA methyltransferases. Annual Review of Biophysical and Biomolecular Structure 24: 293-318

Choong, M.K. and Yan, H. (2008) Multi-scale parametric spectral analysis for exon detection in DNA sequences based on forward-backward linear prediction and singular value decomposition of the double-base curves. Bioinformation 2: 273-278 
Chung, B.Y., Simons, C., Firth, A.E., Brown, C.M., Hellens, R.P. (2006) Effect of 5'UTR introns on gene expression in Arabidopsis thaliana. BMC Genomics 7: 120

Chung, H-R. and Vingron, M. (2009) Sequence-dependent nucleosome positioning. Journal of Molecular Biology 386: 1411-1422.

Cioffi, A., Fleurv, T.J., Stein, A. (2006) Aspects of large-scale chromatin structures in mouse liver nuclei can be predicted from the DNA sequence. Nucleic Acids Research 34: 1974-1981

Cloutier, T.E., Widom, J. (2004) Spontaneous sharp bending of double-stranded DNA. Molecular Cell 14: 335-362

Cohanim, A.B., Kashi, Y., Trifonov, E.N. (2005) Yeast nucleosome DNA pattern: deconvolution from genome sequences of S. cerevisiae. Journal of Biomolecular Structure and Dynamics 22: 687-693

Cohanim, A.B., Kashi, Y., Trifonov, E.N. (2006) Three sequence rules for chromatin. Journal of Biomolecular Structure and Dynamics 23: 559-566

Cohen P., Blackburn E.H. (1998) Two types of telomeric chromatin in Tetrahymena thermophila. Journal of Molecular Biology 280: 327-344

Cokus, S.J., Feng, S., Zhang,X., Chen, Z., Merriman, B., Haudenschild, C.D., Pradhan, S., Nelson, S.F., Pellegrini, M., Jacobsen S.E. (2008) Shotgun bisulphite sequencing of the Arabidopsis genome reveals DNA methylation patterning. Nature 452: $215-219$

Conconi, A., Widmer, R.M., Koller, T., Sogo, J.M. (1989) Two different chromatin structures coexist in ribosomal RNA genes throughout the cell cycle. Cell 57: $753-761$

Dalal, Y., Wang, H., Lindsay, S., Henikoff, S. (2007a) Tetrameric structure of centromeric nucleosomes in interphase Drosophila cells. PLoS Biology 5:e218

Dalal, Y., Furuyama, T., Vermaak, D., Henikoff, S. (2007b) Structure, dynamics, and evolution of centromeric nucleosomes. Proceedings of the National Academy of Sciences of the USA. 104: 15974-15981

Davey, C., Pennings, S., Allan, J. (1997) CpG methylation remodels chromatin structure in vitro. Journal of Molecular Biology 267: 276-288

Davey, C.A. and Richmond, T.J. (2002) DNA-dependent divalent cation binding in the nucleosome core particle. Proceedings of the National Academy of Sciences of the USA. 99: 11169-11174

Davey, C.A., Sargent, D.F., Luger, K., Maeder, A.W., Richmond T.J. (2002) Solvent mediated interactions in the structure of the nucleosome core particle at $1.9 \mathrm{a}$ resolution. Journal of Molecular Biology 319: 1097-1113 
Davey, C., Allan, J. (2003) Nucleosome positioning signals and potential H-DNA within the DNA sequence of the imprinting control region of the mouse Igf $2 \mathrm{r}$ gene. Biochimica et Biophysica Acta 1630: 103-116

Davey, C., Fraser, R., Smolle, M., Simmen, M.M., Allan, J. (2003). Nucleosome positioning signals in the DNA sequence of the human and mouse H19 imprinting control regions. Journal of Molecular Biology 325: 873-887

Davey, C.S., Pennings, S., Reilly, C., Meehan, R.R., Allan J. (2004) A determining influence for $\mathrm{CpG}$ dinucleotides on nucleosome positioning in vitro. Nucleic Acids Research 32:4322-4331

Denisov, D.A., Shpigelman, E.S., Trifonov, E.N. (1997) Protective nucleosome centering at splice sites as suggested by sequence-directed mapping of the nucleosomes. Gene 205: 145-149

Dingwall, C., Lomonossoff, G.P., Laskey, R. A. (1981) High sequence specificity of micrococcal nuclease. Nucleic Acids Research 9: 2659-2673

Doelling, J.H., Gaudino, R.J., Pikaard, C.S. (1993) Functional analysis of Arabidopsis thaliana rRNA gene and spacer promoters in vivo and by transient expression. Proceedings of the National Academy of Sciences of the USA. 90: 75287532

Drew, H.R. and Travers, A.A. (1985) DNA bending and its relation to nucleosome positioning. Journal of Molecular Biology 186: 773-790

Earley, K., Lawrence, R.J., Pontes, O., Reuther, R., Enciso, A.J., Silva, M., Neves, N., Gross, M., Viegas, W., Pikaard C.S. (2006) Erasure of histone acetylation by Arabidopsis HDA6 mediates large-scale gene silencing in nucleolar dominance. Genes and Development 20: 1283-1293

Effron, B. and Tibshirani, R.J. (1994) An Introduction to the Bootstrap. Chapman \& Hall, London, UK, 456 pages

Engelhardt, M. (2007) Choreography for nucleosomes: the conformational freedom of the nucleosomal filament and its limitations. Nucleic Acids Research 35: e106

Fan, Y., Nikitina, T., Morin-Kensicki, E.M., Zhao, J., Magnuson, T.R., Woodcock, C.L., Skoultchi A.I. (2003) H1 linker histones are essential for mouse development and affect nucleosome spacing in vivo. Molecular and Cellular Biology 23: $4559-4572$

Flaus, A., Owen-Hughes, T. (2004) Mechanisms for ATP-dependent chromatin remodelling: farewell to the tuna-can octamer? Current Opinion in Genetics and Development 14: 165-173

Finch, J.T., Noll, M., Kornberg, R.D. (1975) Electron microscopy of defined lengths of chromatin. Proceedings of the National Academy of Sciences of the USA. 72: $3320-3322$ 
Finch, J.T. and Klug, A. (1976) Solenoidal model for superstructure in chromatin. Proceedings of the National Academy of Sciences of the USA. 73: 1897-1901

Finnegan, E.J., Peacock, W.J., Dennis, E.S. (1996) Reduced DNA methylation in Arabidopsis thaliana results in abnormal plant development. Proceedings of the National Academy of Sciences of the USA. 93: 8449-8454

Fransz, P., ten Hoopen, R., Tessadori, F. (2006) Composition and formation of heterochromatin in Arabidopsis thaliana. Chromosome Research 275: 3761-3771

Fraser, R.M., Keszenman-Pereyra, D., Simmen, M.W., Allan, J. (2009) Highresolution mapping of sequence-directed nucleosome positioning on genomic DNA. Journal of Molecular Biology 390: 292-305

Gendrel, A.V., Lippman, Z., Martienssen, R., Colot, V. (2005) Profiling histone modification patterns in plants using genomic tiling microarrays. Nature Methods 2: 213-218

Gilbert, N., Thomson, I., Boyle, S., Allan, J., Ramsahoye, B., Bickmore, W.A. (2007) DNA methylation affects nuclear organization, histone modifications, and linker histone binding but not chromatin compaction. The Journal of Cell Biology 177: $401-411$

Gottesfeld, J.M. and Melton, D.A. (1978) The length of nucleosome-associated DNA is the same in both transcribed and nontranscribed regions of chromatin. Nature 273: 317-319.

Grigoryev, S.A., Spirin, K.S., Krasheninnikov, I.A. (1990) Loosened nucleosome linker folding in transcriptionally active chromatin of chicken embryo erythrocyte nuclei. Nucleic Acids Research 18: 7397-7406

Grant, P.A. (2001) A tale of histone modifications. Genome Biology 2: Reviews 1-6

Grover, A. and Sharma, P.C. (2007) Microsatellite motifs with moderate GC content are clustered around genes on Arabidopsis thaliana chromosome 2. In Silico Biology 7: 0021

Hall, M.A., Shundrovsky, A., Bai, L., Fulbright, R.M., Lis, J.T., Wang, M.D. (2009) High-resolution dynamic mapping of histone-DNA interactions in a nucleosome. Nature Structural and Molecular Biology 16: 124-129

Hsieh, T.F., Fischer, R.L. (2005) Biology of chromatin dynamics. Annual Review of Plant Biology 56: 327-351

Henikoff, S., Furuyama, T., Ahmed, K. (2004) Histone variants, nucleosome assembly and epigenetic inheritance. Trends in Genetics 20: 320-326 
Ioshikhes, I., Bolshoy, A., Derenshteyn, K., Borodovsky, M., Trifonov, E. (1996) Nucleosome DNA sequence pattern revealed by multiple alignment of experimentally mapped sequences. Journal of Molecular Biology 262: 129-139

Jaeger, A.W. and Kuenzle, C.C. (1982) The chromatin repeat length of brain cortex and cerebella neurons changes concomitant with terminal differentiation. The EMBO Journal 7: 811-816

Jeong, Y.M., Mun, J.H., Lee, I., Woo, J.C., Hong, C.B., Kim, S.G. (2006) Distinct roles of the first introns on the expression of Arabidopsis profilin gene family members. Plant Physiology 140: 196-209

Johnson, S.M., Tan, F.J., McCullough, H.L., Riordan, D.P., Fire, A.Z. (2006) Flexibility and constraint in the nucleosome core landscape of Caenorhabditis elegans chromatin. Genome Research 16: 1505-1516

Kahmann, N.H. and Rake, A.V. (1993) Altered nucleosome spacing associated with Down syndrome. Biochemical Genetics 31: 207-214

Kaplan, N., Moore, I.K., Fondufe-Mittendorf, Y., Gossett, A.J., Tillo, D., Field, Y., LeProust, E.M., Hughes, T.R., Lieb, J.D., Widom, J., Segal, E. (2009) The DNA-encoded nucleosome organization of a eukaryotic genome. Nature 458: 362366

Karymov, M.A., Tomschik, M., Leuba, S.H., Caiafa, P., Zlatanova, J. (2001) DNA methylation-dependent chromatin fibre compaction in vivo and in vitro: requirement for linker histone. The FASEB Journal 15:2631-2641

Kato, M., Onishi, Y., Wada-Kiyama, Y., Abe, T., Ikemura, T., Kogan, S., Bolshoy, A., Trifonov, E., Kiyama, R. (2003) Dinucleosome DNA of human K562 cells: experimental and computational characterizations. Journal of Molecular Biology 332: 111-125

Kato, M., Miura, A., Bender, J., Jacobsen, S. E., Kakutani, T. (2003b) Role of CG and Non-CG Methylation in Immobilization of Transposons in Arabidopsis. Current Biology 13: 421-426

Kato, M., Onishi, Y., Wada-Kiyama, Y., Kiyama, R. (2005) Biochemical screening of stable dinucleosomes using DNA fragments from a dinucleosome library. Journal of Molecular Biology 350: 215-227

Kim, J.M., To, T.K., Ishida, J., Morosawa, T., Kawashima, M., Matsui, A., Toyoda, T., Kimura, H., Shinozaki, K., Seki, M. (2008) Alterations of lysine modifications on the histone $\mathrm{H} 3$ N-tail under drought stress conditions in Arabidopsis thaliana. Plant and Cell Physiology 49: 1580-1558

Kiyama, R. and Trifonov, E. (2002) What positions nucleosomes? - A model. FEBS Letters 523: 7-11 
Kogan, S. and Trifinov, E.T. (2005) Gene splice sites correlate with nucleosome positions. Gene 352: 57-62

Kogan, S.B., Kato, M., Kiyama, R., Trifonov, E.T. (2006) Sequence structure of human nucleosomal DNA. Journal of Biomolecular Structure and Dynamics 24: 4348

Köhler, C. and Makarevich, G. (2006) Epigenetic mechanisms governing seed development in plants. EMBO Reports 7: 1223-1227

Längst, G. and Becker, P.B. (2001) ISWI induces nucleosome sliding on nicked DNA. Molecular Cell 8: 1085-1092

Längst, G. and Becker, P.B. (2004) Nucleosome remodelling: one mechanism, many phenomena? Biochimica et Biophysica Acta 1677: 58-63

Lawrence, R.J., Earley, K., Pontes, O., Silva, M., Chen, Z.J., Neves, N., Viegas, W., Pikaard, C.S. (2004) A concerted DNA methylation/histone methylation switch regulates rRNA gene dosage control and nucleolar dominance. Molecular Cell 13: 599-609

Lee, C.K., Shibata, Y., Rao, B., Strahl, B.D., Lieb, J.D. (2004) Evidence for nucleosome depletion at active regulatory regions genome-wide. Nature Genetics 36: 900-905

Lee, W., Tillo, D., Bray, N., Morse, R.H., Davis, R.W., Hughes, T.R., Nislow, C. (2007) A high-resolution atlas of nucleosome occupancy in yeast. Nature Genetics 10: $1235-1244$

Lever, M.A., Th'ng, J.P., Sun, X., Hendzel, M.J. (2000) Rapid exchange of histone H1.1 on chromatin in living human cells. Nature 408: 873-876

Levitsky, V.G., Katokhin, A., Podkolodnaya, O.A., Furman, D.P., Kolchanov, N.A. (2005) NPRD: Nucleosome Positioning Region Database. Nucleic Acids Research 33: Database issue D67-D70.

Li, G., Chandler, S.P., Wolffe, A.P., Hall, T.C. (1998) Architectural specificity in chromatin structure at the TATA box in vivo: nucleosome displacement upon betaphaseolin gene activation. Proceedings of the National Academy of Sciences of the USA. 95: 4772-4777

Lindroth, A.M., Shultis, D., Jasencakova, Z., Fuchs, J., Johnson, L., Schubert, D., Patnaik, D., Pradhan, S., Goodrich, J., Schubert, I., Jenuwein, T., Khorasanizadeh, S., Jacobsen, S.E. (2004) Dual histone H3 methylation marks at lysines 9 and 27 required for interaction with CHROMOMETHYLASE 3. The EMBO Journal 23: 4286-4296

Lippman, Z., Genedrel, A.V., Black, M., Vaughn, M.W., Dedhia, N., McCombie, W.R., Lavine, K., Mittal, V., May, B., Kasschau, K.D., Carrington, J.C., Doerge, 
R.W., Colot, V., Martienssen, R. (2004) Role of transposable elements in heterochromatin and epigenetic control. Nature 430: 471-476

Lister, R., O'Malley, R.C., Tonti-Filippini, J., Gregory, B.D., Berry, C.C., Millar, A.H., Ecker, J.R. (2008) Highly integrated single-base resolution maps of the epigenome in Arabidopsis. Cell 133: 523-536

Lister, R., Pelizzola, M., Dowen, R.H., Hawkins, R.D., Hon, G., Tonti-Filippini, J., Nery, J.R., Lee, L., Ye, Z., Ngo, Q.M., Edsall, L., Antosiewicz-Bourget, J., Stewart, R., Ruotti, V., Millar, A.H., Thomson, J.A., Ren, B., Ecker, J.R. (2009) Human DNA methylomes at base resolution show widespread epigenomic differences. Nature 462, 315-322

Lohr, D., Corden, J., Tatchell, K., Kovacic, R.T., Van Holde, K.E. (1977) Comparative subunit structure of HeLa, yeast, and chicken erythrocyte chromatin. Proceedings of the National Academy of Sciences of the USA. 74: 79-83

Lohr, D. and Van Holde, K.E. (1979) Organisation of spacer DNA in chromatin. Proceedings of the National Academy of Sciences of the USA. 76: 6326-6330

Lorch, Y., LaPointe, J.W., Kornberg, R.D. (1987). Nucleosomes inhibit the initiation of transcription but allow chain elongation with displacement of histones. Cell Research 49: 203-210

Lorch, Y. and Kornberg, R.D. (2004) Isolation and assay of the RSC chromatinremodelling complex from Saccharomyces cerevisiae. Methods in Enzymology 377: 316-322

Lubliner, S. and Segal, E. (2009) Modelling interactions between adjacent nucleosomes improves genome-wide predictions of nucleosome occupancy. Bioinformatics 25: i348-355

Luschnig, C., Bachmair A., Schweizer D. (1993) Intraspecific length heterogeneity of the rDNA-IGR in Arabidopsis thaliana due to homologous recombination. Plant Molecular Biology 22: 543-545

McArthur, M., Thomas, J.O. (1996) A preference of histone H1 for methylated DNA. The EMBO Journal 15: 1705-1714

McStay, B. (2006) Nucleolar dominance: a model for rRNA gene silencing. Genes and Development 20: 1207-1214

Marky, N.L. and Manning, G.S. (1995) A theory of DNA dissociation from the nucleosome. Journal of Molecular Biology 254: 50-61

Marx, K.A., Zhou, Y., Kishawi, I.Q. (2002) Evidence for long poly (dA).poly(dT) tracts in D. discoideum DNA at high frequencies and their preferential avoidance of nucleosomal DNA core regions. Journal of Biomolecular Structure and Dynamics 23: $429-446$ 
Marx, K.A., Hess, S.T., Blake, R.D. (1994) Alignment of (dA).(dT) homopolymer tracts in gene flanking sequences suggests nucleosomal periodicity in $D$. discoideum DNA. Journal of Biomolecular Structure and Dynamics 12: 235-246

Mavrich, T.N., Jiang, C., Ioshikhes, I.P., Li, X., Venters, B.J., Zanton, S.J., Tomsho, L.P., Qi, J., Glaser, R.L., Schuster, S.C., Gilmour, D.S., Albert, I., Pugh, B.F. (2008a) Nucleosome organization in the Drosophila genome. Nature 453: $358-362$

Mavrich, T.N., Ioshikhes, I.P., Venters, B.J., Jiang, C., Tomsho, L.P., Qi, J., Schuster, S.C., Albert, I., Pugh, B.F. (2008b) A barrier nucleosome model for statistical positioning of nucleosomes throughout the yeast genome. Genome Research 18: 1073-1083

Mengeritsky, G. and Trifonov, E.N. (1983) Nucleotide sequence-directed mapping of the nucleosomes. Nucleic Acids Research 11: 3833-3851

Misteli, T., Gunjan, A., Hock, R., Bustin, M., Brown, D.T. (2000) Dynamic binding of histone $\mathrm{H} 1$ to chromatin in living cells. Nature 408:877-881

Noll, M., Zimmer, S., Engel, A., Dubochet, J. (1980) Self-assembly of single and closely spaced nucleosome core particles. Nucleic Acids Research 8: 21-42

Olins, A.L. and Olins, D.E. (1974) Spheroid chromatin units (v bodies). Science 183: $330-332$

Olins, A.L., Senior, M.B., Olins, D.E. (1976) Ultrastructural features of chromatin nu bodies. The Journal of Cell Biology 68: 787-793

Ozsolak, F., Song, J.S., Liu, X.S., Fisher, D.E. (2007) High-throughput mapping of the chromatin structure of human promoters. Nature Biotechnology 25: 244-248

Oudet, P., Gross-Bellard, M., Chambon, P. (1975) Electron microscopic and biochemical evidence that chromatin structure is a repeating unit. Cell 4: 281-300

Parthasarthy, A. and Gopinathan, K.P. (2006) Transcriptional activation of a moderately expressed tRNA gene by positioned nucleosome. Biochemical Journal 396: $439-47$

Peckham, H.E., Thurman, R.E., Fu, Y., Stamatoyannopoulos, J.A., Noble, W.S., Struhl, K., Weng, Z. (2007) Nucleosome positioning signals in genomic DNA. Genome Research 17: 1170-1177

Pennings, S., Allan, J., Davey, C.S. (2005) DNA methylation, nucleosome formation and positioning. Briefings in Functional Genomics and Proteomics 3: 351361

Pruess, A. and Pikaard, C.S. (2007) rRNA gene silencing and nucleolar dominance: Insights into a chromosome-scale epigenetic on/off switch. Biochimica et Biophysica Acta 1769: 383-92 
Rabinowicz, P.D., Palmer, L.E., May, B.P., Hemann, M.T., Low, S.W., McCombie, R., Martienssen, R.A. (2003) Genes and transposons are differentially methylated in plants, but not in mammals. Genome Research 13: 2658-2664

Raisner, R.M. and Madhani, H.D. (2006) Patterning chromatin: form and function for H2A.Z variant nucleosomes. Current Opinion in Genetics and Development 16: $119-124$

Rhodes, D. (1997) Chromatin structure. The nucleosome core all wrapped up. Nature 18: 231-233

Richmond, T.J., Finch, J.T., Rushton, B., Rhodes, D., Klug, A. (1984) Structure of the nucleosome core particle at $7 \AA$ resolution. Nature 17: 532-537

Richmond, T.J. and Davey, C.A. (2003). The structure of DNA in the Nucleosome core. Nature 423: 145-150

Riddle, N.C. and Richards, E.J. (2002) The control of natural variation in cytosine methylation in Arabidopsis. Genetics 162: 355-36

Robinson, P.J. and Rhodes, D. (2006) Structure of the '30 nm' chromatin fibre: a key role for the linker histone. Current Opinion in Structural Biology 16: 336-343

Robinson, P.J., Fairall, L., Huynh, V.A., Rhodes, D. (2006) EM measurements define the dimensions of the "30-nm" chromatin fibre: evidence for a compact, interdigitated structure. Proceedings of the National Academy of Sciences of the USA. 103: 6506-6511

Rose, A.B., Elfersi, T., Parra, G., Korf, I. (2008) Promoter-proximal introns in Arabidopsis thaliana are enriched in dispersed signals that elevate gene expression. Plant Cell 20: 543-551

Routh, A., Sandin, S., Rhodes, D. (2008) Nucleosome repeat length and linker histone stoichiometry determine chromatin fibre structure. Proceedings of the National Academy of Sciences of the USA. 105: 8872-8877

Salganik, R.I, Dudareva, N.A, Kiseleva, E.V. (1991) Structural organization and transcription of plant mitochondrial and chloroplast genomes. Electron Microscopy Reviews 4: 221-247

Satchwell, S.C., Drew, H.R., Travers, A.A. (1986) Sequence periodicities chicken nucleosome core DNA. Journal of Molecular Biology 191: 695-575

Satchwell, S.C., Drew, H.R., Travers, A.A. (1986) Sequence periodicities in chicken nucleosome core DNA. Journal of Molecular Biology 191: 659-675

Satchwell, S.C. and Travers, A.A. (1989) Asymmetry and polarity of nucleosomes in chicken erythrocyte chromatin. The EMBO Journal 8: 229-238 
Schones, D.E., Cui, K., Cuddapah, S., Roh, T.Y., Barski, A., Wang, Z., Wei, G., Zhao, K. (2008) Dynamic regulation of nucleosome positioning in the human genome. Cell 132: 887-98

Schwabish, M.A. and Struhl, K. (2004) Evidence for eviction and rapid deposition of histones upon transcriptional elongation by RNA polymerase II. Molecular and Cell Biology 24: 10111-10117

Segal, E., Fondufe-Mittendorf, Y., Chen, L., Thastrom, A., Field, Y., Moore, I.K., Wang, J.P.Z., Widom, J. (2006) A genomic code for nucleosome positioning. Nature 442: 772-778

Saleh, A., Alvarez-Venegas, R., Yilmaz, M., Le, O., Hou, G., Sadder, M., AlAbdallat, A., Xia, Y., Lu, G., Ladunga, I., Avramova, Z. (2008) The Highly Similar Arabidopsis Homologs of Trithorax ATX1 and ATX2 Encode Proteins with Divergent Biochemical Functions. Plant Cell 20: 568-579.

Seoighe, C., Gehring, C., Hurst, L.D. (2005) Gametophytic selection in Arabidopsis thaliana supports the selective model of intron length reduction. PLoS Genetics 1: e13

Sheather, S.J. and Jones, M.C. (1991) A reliable data-based bandwidth selection method for kernel density estimation. Journal of the Royal Statistical Society, Series B. 53: $683-690$

Shindo, C., Lister, C., Crevillen, P., Nordborg, M., Dean, C. (2006) Variation in the epigenetic silencing of FLC contributes to natural variation in Arabidopsis vernalization response. Genes and Development 20: 3079-3083

Shivaswamy, S., and Bhargava, P. (2006) Positioned nucleosomes due to sequential remodelling of the yeast U6 small nuclear RNA chromatin are essential for its transcriptional activation. Journal of Biological Chemistry 281: 10461-10472

Shrader, T.E., and Crothers, D.M. (1989) Artificial nucleosome positioning sequences. Proceedings of the National Academy of Sciences of the USA. 86: 74187422

Smith, T.F., and Waterman, M.S. (1981) Identification of Common Molecular subsequences. Journal of Molecular Biology 147: 195-197

Staynov, D.Z. and Proykova, Y.G. (2008) The sequentiallity of nucleosomes in the $30 \mathrm{~nm}$ chromatin fibre. FEBS Journal 275: 3761-3771.

Stein, A. and Bina M. (1999) A signal encoded in vertebrate DNA that influences nucleosome position and alignment. Nucleic Acids Research 27: 848-853

Strohner R., Wachsmuth, M., Dachauer, K., Mazurkiewicz, J., Hochstatter, J., Rippe, K., Längst, G. (2005) A 'loop recapture' mechanism for ACF-dependent nucleosome remodelling. Nature Structural and Molecular Biology 12: 683-690 
Tanaka, S., Zatchej, M., Thoma, F. (1992) Artificial nucleosome positioning sequences tested in yeast minichromosomes: a strong rotational setting is not sufficient to position nucleosomes in vivo. The EMBO Journal 11: 1187-1193

Tanaka, T., Koyanagi, K.O., Itoh, T. (2009) Highly diversified molecular evolution of downstream transcription start sites in rice and Arabidopsis. Plant Physiology 149: 1316-1324

Tariq, M., Saze, H., Probst, A.V., Lichota, J., Habu, Y., Paszkowski, J. (2003) Erasure of $\mathrm{CpG}$ methylation in Arabidopsis alters patterns of histone $\mathrm{H} 3$ methylation in heterochromatin. Proceedings of the National Academy of Sciences of the USA. 100: $8823-8827$

Tchurikov, N. A. (2005) Molecular mechanisms of epigenetics. Biochemistry (Moscow) 70: 406-423

Tienari, P.J., Kuokkanen, S., Pastien, T., Wikstrom, J., Sajantila, A., SandbergWollheim, M., Palo, J., Peltonen, L. (1998) Golli-MBP gene in multiple sclerosis susceptibility. Journal of Neuroimmunology 81: 158-167

Ull, M.A. and Franco, L. (1986) The nucleosome repeat length of pea (Pisum savitum) chromatin changes during germination. Plant Molecular Biology 7: 25-32

Unfried, I. and Gruendler, P. (1990) Nucleotide sequence of the 5.8S and 25S rRNA genes and of the internal transcribed spacers from Arabidopsis thaliana. Nucleic Acids Research 18: 4011

Villar, C.B.R., Erilova, A., Makarevich, G., Trösch R., Köhler, C. (2009) Control of PHERES1 Imprinting in Arabidopsis by Direct Tandem Repeats. Molecular Plant 2: $654-660$

Virstedt, J., Berge, T., Henderson, R.M., Waring, M.J., Travers, A.A. (2004) The influence of DNA stiffness upon nucleosome formation. Journal of Structural Biology 148: 66-85

Vignali, M., Hassan, A.H., Neely, K.E., Workman, J.L. (2000) ATP-dependent chromatin remodelling complexes. Molecular and Cell Biology 20:1899-910

Vinogradov, A.E. (2005) Non-coding DNA, isochores and gene expression: nucleosome formation potential. Nucleic Acids Research 33: 559-63

Waddington, C.H. (1953) Genetic Assimilation of an Acquired Character. Evolution 7: 118-126

Wambutt, R., Murphy, G., Volckaert, G., Pohl, T., Düsterhöft, A., Stiekema, W., Entian, K.D., Terryn, N., Harris, B., Ansroge, W., Brandt, P., Grivell, L., Rieger, M., Weichselgartner, M., de Simone, V., Obermaier, B., Mache, R., Müller, M., Kreis, M., Delseny, M., Puigdomenech, P., Watson, M., Schmidtheini, T., Reichert, B., Portatelle, D., Perez-Alonso, M., Bountry, M., Bancroft, I., Vos, P., Hoheisel, J., Zimmermann, W., Wedler, H., Ridley, P., 
Langham, S.A., McCullagh, B., Bilham, L., Robben, J., Van der Schueren, J., Grymonprez, B., Chuang, Y.J., Vandenbussche, F., Braeken, M., Weltjens, I., Voet, M., Bastiens, I., Aert, R., Defoor, E., Weitzenegger, T., Bothe, G., Rose, M. (2000) Progress in Arabidopsis genome sequencing and functional genomics. Journal of Biotechnology 78: 281-292

Wang, J.P., Fondufe-Mittendorf, Y., Xi, L., Tsai, G.F., Segal, E., Widom, J. (2008) Preferentially quantized linker DNA lengths in Saccharomyces cerevisiae. PLoS Computational Biology 4: e1000175

Wendel, J.F., Cronn, R.C., Alvatez, I., Liu, B., Small, R.L., Senchina, D.S. (2002) Intron size and genome size in plants. Molecular Biology and Evolution 19: 2346-2352

Weintraub, H. (1978) The nucleosome repeat length increases during erythropoiesis in the chick. Nucleic Acids Research 5: 1179-1188

Widlak, P. and Garrard, W.T. (2006) Unique features of the apoptotic endonuclease DFF40/CAD relative to micrococcal nuclease as a structural probe for chromatin Biochemistry and Cell Biology 84: 405-410

Widlund, H., Cao, H., Simonsson, S., Magnusson, E., Simonsson, T., Nielsen, P., Kahn, J., Crothers, D., Kubista, M. (1997) Identification and characterisation of genomic nucleosome-positioning sequences. Journal of Molecular Biology 267: 807817

Widom, J. (1992) A relationship between the helical twist of DNA and the ordered positioning of nucleosomes in all eukaryotic cells. Proceedings of the National Academy of Sciences of the USA. 89: 1095-1099

Wierzbicki, A.T., and Jerzmanowski, A. (2005) Suppression of histone H1 genes in Arabidopsis results in heritable developmental defects and stochastic changes in DNA methylation. Genetics 169: 997-1008

Winston, F. and Carlson, M. (1992) Yeast SNF/SWI transcriptional activators and the SPT/SIN chromatin connection. Trends in Genetics 8: 387-391

Wong, H., Victor, J.M., Mozziconacci, J. (2007) An all-atom model of the chromatin fibre containing linker histones reveals a versatile structure tuned by the nucleosomal repeat length. PLoS One 2: e877

Woo, H.R. and Richards, E.J. (2008) Natural variation in DNA methylation in ribosomal RNA genes of Arabidopsis thaliana. BMC Plant Biology 8:92

Woodcock, C.L. and Horowitz, R.A. (1997) Electron microscopy of chromatin. Methods 12: 84-95

Woodcock C.L., Skoultchi, A.I., Fan, Y. (2006) Role of linker histone in chromatin structure and function: $\mathrm{H} 1$ stoichiometry and nucleosome repeat length Chromosome Research 14: 17-25 
Workman, J.L. (2006) Nucleosome displacement in transcription. Genes and Development 20: 2009-2017

Yuan, G.C., Liu, Y.J., Dion, M.F., Slack, M.D., Wu, L.F., Altscluler, S.J., Rando, O.J. (2005) Genome-scale identification of nucleosome positions in S. cerevisiae. Science 309: 626-630

Yang, J.G., Madrid, T.S., Sevastopoulos, E., Narlikar, G.J. (2006) Chromatin remodelling enzyme ACF is an ATP-dept DNA length sensor that regulated nucleosome spacing. Nature Structural and Molecular Biology 13: 1078-83

Zhang, X., Yazaki, J., Sundaresan, A., Cokus, S., Chan, S.W., Chen, H., Henderson, I.R., Shinn, P., Pellegrini, M., Jacobsen, S.E., Ecker, J.R. (2006) Genome-wide high-resolution mapping and functional analysis of DNA methylation in Arabidopsis. Cell 126: 1189-1201

Zhang, L, Wu, C., Carta, R., Zhao, H. (2007) Free energy of DNA duplex formation on short oligonucleotide microarrays Nucleic Acids Research 35: e18.

Zhang, Y, Shin, H., Song, J.S., Lei, Y., Liu, X.S. (2008). Identifying Positioned Nucleosomes with Epigenetic Marks in Human from ChIP-Seq. BMC Genomics 9: 537

Zilberman, D. and Henikoff, S. (2005) Epigenetic inheritance in Arabidopsis: selective silence. Current Opinion in Genetics and Development 15: 1-6

Zilberman, D., Coleman-Derr, D., Ballinger T., Henikoff S. (2008) Histone H2A.Z and DNA methylation are mutually antagonistic chromatin marks. Nature 456: $125-129$ 